RCEIVE:

N10Y 14 1996

OSTI

Tank SY-102 Waste Retrieval

Assessment: Rheological

Measurements and Pump Jet

Mixing Simulations

Y. Onishi

R. Shekarriz

J. Liu

K. P. Recknagle

Y. L. Chen

P. A. Smith

D. R. Rector

J. D. Hudson

September 1996

Prepared for the U.S. Department of Energy under Contract DE-AC06-76RLO 1830

Pacific Northwest National Laboratory -Operated for the U.S. Department of Energy by Battelle 


\section{DISCLAIMER}

This report was prepared as an account of work sponsored by an agency of the United States Government. Neither the United States Government nor any agency thereof, nor Battelle Memorial Institute, nor any of their employees, makes any warranty, express or implied, or assumes any legal liability or responsibility for the accuracy, completeness, or usefulness of any information, apparatus, product, or process disclosed, or represents that its use would not infringe privately owned rights. Reférence herein to any specific commercial product, process, or service by trade name, trademark, manufacturer, or otherwise does not necessarily constitute or imply its endorsement, recommendation, or favoring by the United States Govemment or any agency thereof, or Battelle Memorial Institute. The views and - opinions of authors expressed herein do not necessarily state or reflect those of the United States Govemment or any agency thereof.

\section{PACIEIC NOETHWEST NATIONAL LABORATORY \\ $\because \quad$ Operated by \\ BATTELLE \\ for the \\ UNITED STATES DEPARTMENT OF ENERGY under Contract DE-AC06-76RLO -1830.}

- Printed in the United States of America

Available to DOE and DOE contractors from the

Office of Scientific and Technical Informiation, P.O. Box 62, Oak Ridge, TN 3783i; prices available from (615) 576-8401.

Available to the public from the National Technical information Senvice, -

U.S. Department of Commerce, 5285 Port Royal Rd,, Springfield, VA 22161 


\title{
Tank SY-102 Waste Retrieval Assessment: Rheological Measurements and Pump Jet Mixing Simulations
}

\author{
Y. Onishi \\ R. Shekarriz \\ K. P. Recknagle \\ P. A. Smith \\ J. Liu \\ Y. L. Chen \\ D. R. Rector \\ J. D. Hudson
}

September 1996

Prepared for

the U.S. Department of Energy

under Contract DE-AC06-76RLO 1830

Pacific Northwest National Laboratory

Richland, Washington 99352 



\section{DISCLAIMER}

Portions of this document may be illegible in electronic image products. Images are produced from the best available original document. 


\section{Executive Summary}

Wastes stored in Hanford Tank 241-SY-10 are planned to be retrieved from the tank and transferred to 200 East Area through the new pipeline Replacement Cross Site Transfer System (RCSTS). This report describes results of the second phase (the detailed assessment) of a the SY-102 waste retrieval study, which is a part of the efforts to establish a technical basis for mobilization of the slurry, waste retrieval, and slurry transport. This second-phase study was performed to close uncertainty issues on Tank SY-102 waste retrieval identified under the previous preliminary assessment (Onishi and Hudson 1996). The uncertainties on the preliminary assessment were primarily related to lack of data on waste characteristics and the assumption of the tank being fully mixed. To resolve these issues, this Phase II study 1) identified solid phases of the SY-102 waste by using transmission electron microscopy (TEM), 2) conducted laboratory experiments to measure rheology, zeta potential to evaluate solid agglomeration, and sedimentation, 3) measured particle size distributions of the wastes, and 4) simulated time-varying, three-dimensional, tank pump jet mixing.

The Phase II study results confirms the validity of the assumptions and parameter selections used in the preliminary assessment (Onishi and Hudson 1996) and supports its conclusion that the SY-102 waste mixing and heating do not adversely impact the waste retrieval operation. Specific conclusions of this study are briefly summarized below.

The Transmission Electron Microscopy results show that the morphology of the agglomerates is dominated by large size $(2-5 \mu \mathrm{m} \times 10-30 \mu \mathrm{m}) \mathrm{NaOH}$ rods surrounded by finer primary particles of various materials. A large population of amorphous silica and iron hydroxide primary particles in the nanometer size range was observed. Also, needle-like $\mathrm{FeO}(\mathrm{OH})$ agglomerates and sharp-edged plate-like $\gamma-\mathrm{Al}_{2} \mathrm{O}_{3}$ particles were found. Irregular $U$-containing agglomerates and rectangular $\mathrm{Al} / \mathrm{Cr}-$ containing particles were also present. The major crystalline phases observed include: micrometersized $\gamma-\mathrm{Al}_{2} \mathrm{O}_{3}$, needle-like $\mathrm{FeO}(\mathrm{OH})$ and $\mathrm{Al}_{2} \mathrm{Si}_{4} \mathrm{O}_{10}$. The minor crystalline phases observed were rodlike particles of hydroxylapatite, $\mathrm{Ca}_{5}(\mathrm{PO})_{3}(\mathrm{OH})$ and single crystalline gibbsite, $\mathrm{Al}(\mathrm{OH})_{3}$. $\mathrm{t}$ rod-like particles of hydroxylapatite, $\mathrm{Ca}_{5}(\mathrm{PO})_{3}(\mathrm{OH})$ and single crystalline gibbsite, $\mathrm{Al}(\mathrm{OH})_{3}$. The preliminary assessment study (Onishi and Hudson 1996) assumed the all Al solids to be gibbsite for the chemical reaction modeling. The current TEM identified $\mathrm{Al}$ solids to be $\gamma-\mathrm{Al}_{2} \mathrm{O}_{3}, \mathrm{Al}_{2} \mathrm{Si}_{4} \mathrm{O}_{10}$, and gibbsite. Gibbsite and $\gamma-\mathrm{Al}_{2} \mathrm{O}_{3}$ are chemically similar. Moreover, existence of $\gamma-\mathrm{Al}_{2} \mathrm{O}_{3}$ would slightly increase the amount of water in the solution, as compared with gibbsite. Moreover, since the sludge has a much smaller amount of Si than the amount of $\mathrm{Al}$, the majority of $\mathrm{Al}$ is not expected to form a solid with $\mathrm{Si}$. Thus, the preliminary assessment with gibbsite may have slightly underestimated the amount of solids being dissolved, resulting in a slightly more conservative estimate (a worse case) for the retrieval operation.

The photon correlation spectroscopy size analysis results show a volume-averaged mean of $6.435 \pm 1.025 \mu \mathrm{m}(0.422 \pm 0.04 \mu \mathrm{m}$ number-averaged mean) for particles dispersed in deionized water and a volume-averaged mean of $7.245 \pm 1.16 \mu \mathrm{m}(0.866 \pm 0.087 \mu \mathrm{m}$ number-averaged mean $)$ for $1 \underline{\mathrm{M}}$ $\mathrm{NaNO}_{3}$ solution. These results suggest that as the solution becomes more ionic, the size of the aggregates tend to grow. Essentially, producing a strongly attractive system with the $1 \underline{\mathrm{M}}$ electrolyte concentration results in more aggregation. Further, it was-found that sonication tends to severely reduce the volume-averaged mean and has little effect on the number-averaged mean, suggesting that sonication breaks up the largest agglomerates in the size distribution.

The zeta potential measurements for the SY-102 waste sample show that the isoelectric point is approximately at $\mathrm{pH}$ of 3.2. Parks (1965) has shown that the isoelectric points of metal oxide/ hydroxide phases vary according to the binding force and correspondingly to the phase. Specific- ally, $\mathrm{RO}_{2}$ phases such as silica possess isoelectric points typically in the range of pH's between 2 to 4 . 
Clearly, the silica phase is controlling the surface charge development of the SY-102 waste as indicated by the zeta potential. This is consistent with the TEM results that show a significant presence of the colloidal amorphous silica in the aggregates.

Rheological measurements of the SY-102 waste show a rheopectic behavior at low shear rates $\left[<O\left(1 \mathrm{~s}^{-1}\right)\right]$ and a thixotropic behavior at high shear rates $\left[\sim \mathrm{O}\left(10 \mathrm{~s}^{-1}\right)\right]$. These results were consistent with the observations about the role of silica particles in surface charge of the agglomerates. These results suggest that if the slurry is moving at a slow enough rate, aggregation of the primary particles and smaller aggregates, to form larger aggregates, is promoted. In fact, the continuous increase in the viscosity at the lowest shear rate suggests that gelation may be possible, if operated at these shear rates for a sustained period of time. Gelation can be avoided by operating at shear rates higher than $1 \mathrm{~s}^{-1}$. Since the period of jet rotaion in SY-102 is expected to be two minutes, gelation is considered not to be important. As the fluid is sheared faster, the rate of disaggregation due to higher shearing stresses increases and balances the rate of agglomeration at shear rate $\sim O\left(1 \mathrm{~s}^{-1}\right)$. At a shear rate of $10 \mathrm{~s}^{-1}$, the disaggregation rate exceeds the agglomeration rate and as a result a thixotropic behavior is observed. And finally, at a high enough rate, the agglomerates have reached an equilibrium size distribution, mainly controlled by the chemistry.

Rheograms of the undiluted sample show that the material has strong pseudoplasticity (shearthinning) behavior. Two constitutive models can be used to represent the behavior of the undiluted waste: power-law, where the consistency factor is $1.5 \mathrm{~Pa} . \mathrm{s}^{\mathrm{n}}$ and behavior index is 0.19 ; and Bingham plastic, where the yield stress is $2.7 \mathrm{~Pa}$ and viscosity is $5 \mathrm{mPa}$. Further, the relative viscosity of the sample was found to decrease. when diluted with $\mathrm{NaNO}_{3}$ solution (same ionic strength was maintained). The relation between the relative viscosity and solids concentration was found to be consistent with the empirical model by Chong et al. (1971). Based on the behavior of non-colloidal suspensions, we speculate that the reduced viscosity associated with the higher shear rate data (10 and $100 \mathrm{~s}^{-1}$ ) is as a result of increase in the maximum packing fraction of the slurry. As the slurry is sheared at higher rates, the agglomerates break up and broaden the size distribution. This broadening would increase both the ratio of larger to smaller particle diameters and the volume fraction of smaller particles in the suspension, in turn increasing the maximum packing fraction of the suspension. Using this model, the $10 \mathrm{~s}^{-1}$ and $100 \mathrm{~s}^{-1}$ data seem to fit closely to $5 \%$ and $14 \%$ increase in the maximum packing fractions over the low shear rate limit, respectively. These viscosities were found to be significantly lower'than the previous results reported by DiCenso (1995). As a result, DiCenso's values may be used for conservative modeling of the mixing process.

The current study estimated that the viscosity of the sludge/supernate mixture is approximately $2 \mathrm{cP}$, while the preliminary assessment used viscosity of 4.8-14 cP to determine an expected pressure drop in the slurry pipeline. Thus, the preliminary assessment for the pipeline transport was conservative. Thus, the current study supports the preliminary assessment conclusion that the sludge/ supernate mixture will be successfully transferred through RCSTS.

The compressive yield stress was found from the centrifugation results. A model was developed for slurry compaction which predicted the slurry behavior within 5\% of the measured data. The compressive strength of the sludge at the bottom of the tank was then estimated to be approximately $80.6 \mathrm{gm} / \mathrm{cm}^{2}$ (7.9 $\mathrm{kPa}$ or $\left.1.2 \mathrm{psi}\right)$. This parameter is considered relevant due to the fact that many of the stresses on the sludge that cause the sludge to yield are in the normal compressive mode rather than shear. Note that this value is significantly higher than the shear strength of the waste, which implies that, depending on what failure theory is used, the compressive strength might be the dominant resistive strength of the material. In this study, failure is assumed to be entirely due to shear stresses. Further, the volume fraction of the sludge at a depth of $\sim 45^{\prime \prime}$ below the sludge-supernatant layer interface was estimated to be $51 \%$. This value is what the input to the computational modeling is based on.

The objective of the Tank SY-102 modeling was to determine whether two pumps having four rotating $60-\mathrm{ft} / \mathrm{s}$ jets located at $20 \mathrm{ft}$ off center in Tank SY-102 can sufficiently mix the sludge with 
supernate for the waste retrieval. We used the three-dimensional computer code TEMPEST to simulate the movements of supernate and the sludge having seven different solid size fractions under ten different conditions. These ten cases include two sets of viscosity variations, two sets of initial sludge distributions, two different pump locations, and four jet velocities. TEMPEST predicted that the rotating jets with $0.5 \mathrm{rpm}$ are mixing sludge and supernate rapidly and effectively. They achieve much of the mixing in 10-20 minutes (20-40 sweeps by the rotating jets) within most areas of the tank, and the final, quasi-steady-state is reached in less than two hours, regardless of different viscosity values used in the modeling.

Thus, the current tank modeling effort confirms the validity of the chemical modeling of the preliminary assessment and supports its conclusion that the SY-102 waste mixing and heating do not adversely impact the waste retrieval operation. Moreover the preliminary assessment concludes that main solids to have dissolution/precipitation reactions were $\mathrm{Na}$-containing solids, especially $\mathrm{NaNO}_{3}(\mathrm{~s})$. Since $\mathrm{NaNO}_{3}(\mathrm{~s})$ is expected to be dissolved or precipitated between 15 minutes and one hour, the current model prediction that the bulk of the mixing will be achieved in 10 minutes to two hours supports the use of the equilibrium chemical modeling performed under the preliminary assessment.

Although the current TEMPEST code has limitations of handling yield stress and formation/ break-up of aggregates, since the SY-102 sludge is generally believed to have very small yield stress, the assumption of no yield stress imposed in this modeling was judged reasonable. Should there actually be a significant yield stress in the sludge, there may be more resistance to sludge mobilization than currently modeled. This study also provided estimates of the potential sludge mobilization areas with various yield stress levels.

The SY-102 model also predicted that the solids are mostly suspended from the tank bottom by the direct hits by'the rotating jets and are then moved by large scale vertical and horizontal eddies induced in the tank. Thus, the mixing efficiency is related to the frequency of the jet rotation, i.e., the faster the rotation, the shorter the required time to achieve near full mixing, as long as the jets are hitting each tank spot with a reasonable duration during the rotation. 



\section{Contents}

Summary $\ldots \ldots \ldots \ldots \ldots \ldots \ldots \ldots \ldots \ldots \ldots \ldots \ldots \ldots \ldots \ldots \ldots \ldots \ldots \ldots \ldots \ldots \ldots \ldots \ldots$

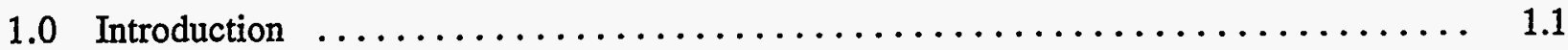

2.0 Rheological Measurements and Analysis $\ldots \ldots \ldots \ldots \ldots \ldots \ldots \ldots \ldots \ldots \ldots \ldots \ldots \ldots$

2.1 Experimental Procedure $\ldots \ldots \ldots \ldots \ldots \ldots \ldots \ldots \ldots \ldots \ldots \ldots \ldots \ldots \ldots \ldots \ldots \ldots \ldots$

2.1.1 Solid Phase Morphological Characterization $\ldots \ldots \ldots \ldots \ldots \ldots \ldots \ldots .1$

2.1.2 Particle Size Distribution $\ldots \ldots \ldots \ldots \ldots \ldots \ldots \ldots \ldots \ldots \ldots \ldots \ldots .1$

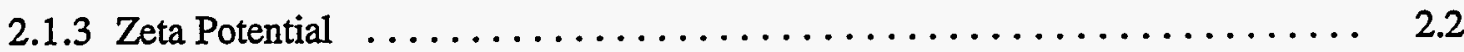

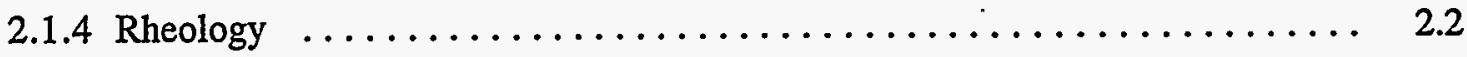

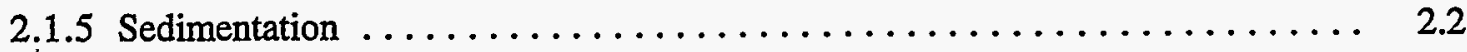

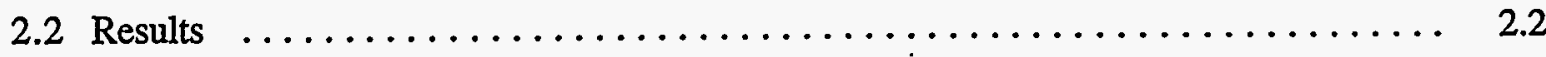

2.2.1 TEM Characterization Results $\ldots \ldots \ldots \ldots \ldots \ldots \ldots \ldots \ldots \ldots \ldots \ldots \ldots \ldots \ldots$

2.2.2 Particle Size Distribution $\ldots \ldots \ldots \ldots \ldots \ldots \ldots \ldots \ldots \ldots \ldots \ldots \ldots \ldots \ldots \ldots$

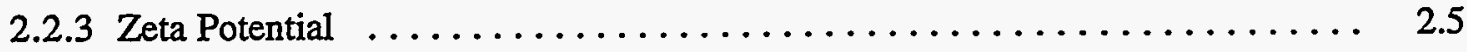

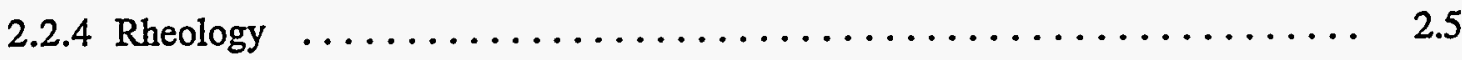

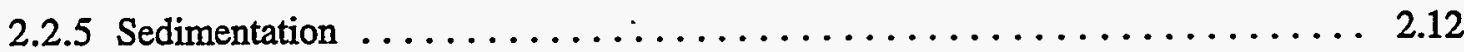

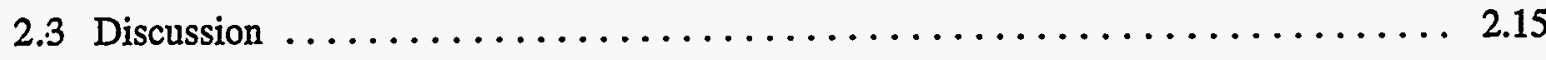

3.0 Pump Jet Mixing Simulations $\ldots \ldots \ldots \ldots \ldots \ldots \ldots \ldots \ldots \ldots \ldots \ldots \ldots \ldots \ldots \ldots \ldots \ldots \ldots$

3.1 SY-102 Tank Wastes and Retrieval $\ldots \ldots \ldots \ldots \ldots \ldots \ldots \ldots \ldots \ldots \ldots \ldots \ldots \ldots \ldots$

3.2 Tank SY-102 Model Setup with the Tempest Code $\ldots \ldots \ldots \ldots \ldots \ldots \ldots \ldots$

3.3 Model Applications and Result Evaluations $\ldots \ldots \ldots \ldots \ldots \ldots \ldots \ldots \ldots$

3.3 .1 Grid Resolutions $\ldots \ldots \ldots \ldots \ldots \ldots \ldots \ldots \ldots \ldots \ldots \ldots \ldots \ldots \ldots$

3.3.2 Simulation Results of Case 1: Base Case $\ldots \ldots \ldots \ldots \ldots \ldots \ldots \ldots$

3.3.3 Case 2: Fully Mixed Case $\ldots \ldots \ldots \ldots \ldots \ldots \ldots \ldots \ldots \ldots \ldots \ldots \ldots \ldots \ldots \ldots \ldots \ldots$

3.3.4 Cases 3 and 4: High Viscosity Stratified and Fully Mixed Sludge Cases . . 3.20

3.3.5 Cases 5 and 6: Center-Located Pump with Stratified and Fully

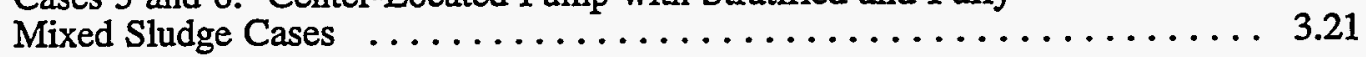

3.3.6 Cases 7 Through 10: Reduced Jet Velocity Cases $\ldots \ldots \ldots \ldots \ldots \ldots \ldots$ 
4.0 Summary and Conclusions $\ldots \ldots \ldots \ldots \ldots \ldots \ldots \ldots \ldots \ldots \ldots \ldots \ldots \ldots \ldots \ldots \ldots \ldots \ldots$

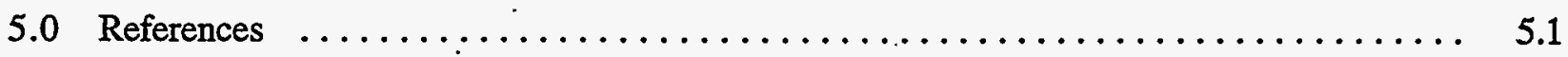

Appendix A: Electron Microscopy Results $\ldots \ldots \ldots \ldots \ldots \ldots \ldots \ldots \ldots \ldots \ldots \ldots$ A.1

Appendix B: Particle Size Analysis Results $\ldots \ldots \ldots \ldots \ldots \ldots \ldots \ldots \ldots \ldots \ldots \ldots \ldots \ldots$

Appendix C: Pump Jet Mixing Simulation Results $\ldots \ldots \ldots \ldots \ldots \ldots \ldots \ldots \ldots \ldots \ldots$ C.1 


\section{Figures}

2.1 Zeta Potential as a Function of $\mathrm{pH}$ for Tank SY-102 Waste Slurry $\ldots \ldots \ldots \ldots \ldots$

2.2 Rheopexy or Antithixotropic Behavior of Tank SY-102 Waste at Low Shear Rates $\ldots \ldots \quad 2.6$

2.3 Thixotropic Behavior of Tank SY-102 Waste at High Shear Rates $\ldots \ldots \ldots \ldots . \ldots$

2.4 Rheograms of Diluted and Undiluted Tank SY-102 Waste $\ldots \ldots \ldots \ldots \ldots \ldots \ldots$

2.5 Viscosity of Diluted and Undiluted Tank SY-102 Waste $\ldots \ldots \ldots \ldots \ldots \ldots \ldots$

2.6 Effective Viscosity of Tank SY-102 Waste as a Function of Solids Concentration . . . . . 2.11

2.7 Compaction Results for Tank $S Y-102$ Waste $\ldots \ldots \ldots \ldots \ldots \ldots \ldots \ldots \ldots \ldots \ldots$

2.8 Compressive Yield Stress as a Function of Solids Volume Fraction for

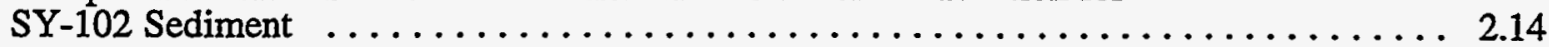

2.9 Solids Density Profiles versus Distance From the Top of the Sediment $\ldots \ldots \ldots 2.15$

3.1 Variation of Viscosity with Solid Concentrations for Sludge Viscosity of

$12 \mathrm{~Pa}$-s and $\mathrm{C}_{\mathrm{vmax}}$ of 0.53 , with Some Measured Viscosity $\ldots \ldots \ldots \ldots \ldots \ldots \ldots \ldots$

-3.2 Variation of Viscosity with Solid Concentrations for Sludge Viscosity of

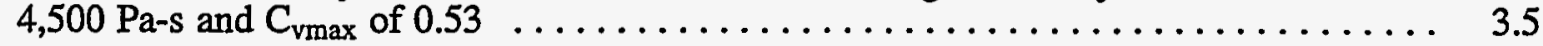

3.3 Initial Distributions of Velocity $(\mathrm{m} / \mathrm{s})$ on Tank Bottom and Three Dimensional

Distribution of Solid 7 Volume Fraction for Case $1 \ldots \ldots \ldots \ldots \ldots \ldots \ldots \ldots \ldots$

3.4 Predicted Distributions of Velocity on Tank Bottom and Three Dimensional

Distribution of Solid 7 Volume Fraction at Three Simulation Minutes, Case 1

3.5 Predicted Distributions of Velocity $(\mathrm{m} / \mathrm{s})$ on Tank Bottom and Three Dimensional Distribution of Solid 7 Volume Fraction at Six Simulation Minutes for Case $1 \ldots \ldots .12$

3.6 Predicted Distributions of Velocity $(\mathrm{m} / \mathrm{s})$ on Tank Bottom and Three Dimensional Distribution of Solid 7. Volume Fraction at Ten Simulation Minutes for Case 1 . . . . . 3.13

3.7 Predicted Distributions of Velocity $(\mathrm{m} / \mathrm{s})$ on Tank Bottom and Three Dimensional Distribution of Solid 7 Volume Fraction at Two Simulation Hours for Case 1 . . . . . . 3.14

3.8 Estimated Tank Bottom Areas for Possible Sludge Mobilization at Different Velocity and Yield Stress Values for Case $1(60 \mathrm{ft} / \mathrm{s}$ jets $) \ldots \ldots \ldots \ldots \ldots \ldots \ldots \ldots$

3.9 Estimated Tank Bottom Areas for Possible Sludge Mobilization at Different Velocity and Yield Stress Values for Case $7(45 \mathrm{ft} / \mathrm{s}$ jets $) \ldots \ldots \ldots \ldots \ldots \ldots \ldots \ldots \ldots \ldots \ldots \ldots .25$

3.10 Estimated Tank Bottom Areas for Possible Sludge Mobilization at Different Velocity and Yield Stress Values for Case 8 and $9(30 \mathrm{ft} / \mathrm{s}$ jets $) \ldots \ldots \ldots \ldots \ldots \ldots \ldots$

3.11 Estimated Tank Bottom Areas for Possible Sludge Mobilization at Different Velocity and Yield Stress Values for Case 10 (15 ft/s jets) 
A.1 Low-Magnification TEM Image of SY-102 Waste Sample $(2000 \mathrm{X}), \ldots \ldots \ldots \ldots$. . . . .

A.2 TEM Image of g-A12O3, Amorphous Silica, and Amorphous Iron Hydroxide Agglomerates

A.3 TEM Image of Rod-Like FeOOH, g-Al2O3, and Amorphous Iron Hydroxide Agglomerates

A.4 TEM Image of Amorphous U-Containing Particles $\ldots \ldots \ldots \ldots \ldots \ldots \ldots \ldots \ldots$

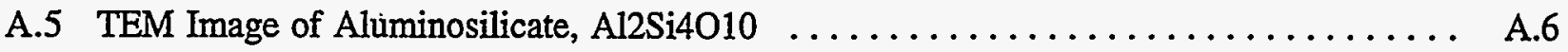

A.6 TEM Image of Particle Containing Both $\mathrm{Al}$ and $\mathrm{Cr} \ldots \ldots \ldots \ldots \ldots \ldots \ldots \ldots$

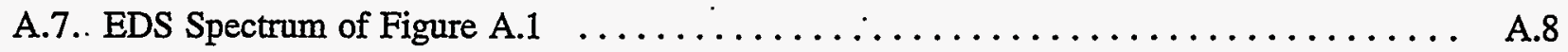

A.8. EDS Spectrum of $\mathrm{NaOH}$ Rod-Like Particles in Figure A.1 $\ldots \ldots \ldots \ldots \ldots \ldots \ldots \ldots$ A.8

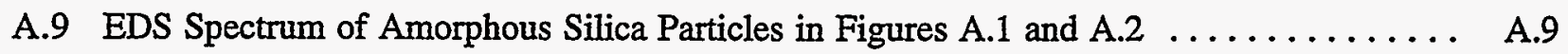

A.10 EDS Spectrum of $\mathrm{g}-\mathrm{Al} 2 \mathrm{O} 3$ as Shown in Figures A.2 and A.3 $\ldots \ldots \ldots \ldots \ldots \ldots$

A.11 EDS Spectrum of Iron Hydroxide Particles Shown in Figures A.1, A.2, and A.3 . . . . A.10

A.12 EDS Spectrum of Amorphous U-Containing Particles $\ldots \ldots \ldots \ldots \ldots \ldots \ldots \ldots$. . . . . .

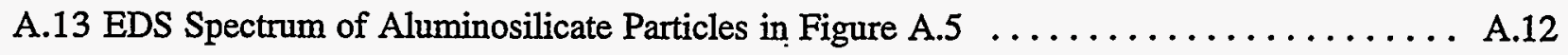

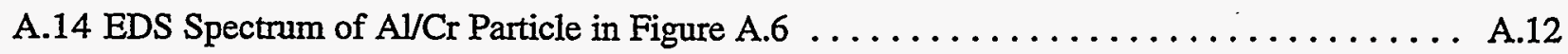

A.15 Selected Area Diffraction (SAD) Pattern from g-A12O3 Shown in Figure A.2 . . . . . A.13

A.16 SAD Pattern from FeOOH Particles Shown in Figure A.3 . . . . . . . . . . . A.14

A.17 SAD Pattern from Al2Si4O10 Particles Shown in Figure A.5 . . . . . . . . . A.15

B.1 Particle Size Distribution of SY-102 Sample in DI Water (s96r000511 Sample) $\ldots . . . \quad$ B.1

B.2. Particle Size Distribution of SY-102 Sample in DI Water Sonicated for

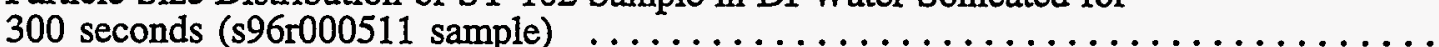

B.2

B.3 Particle Size Distribution of SY-102 Sample in 0.1 M NaNO3 Solution

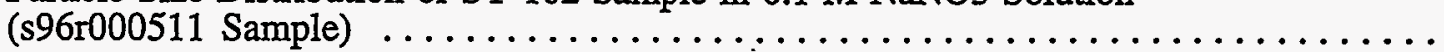

B.4 Particle Size Distribution of SY-102 Sample in 0.1 M NaNO3 Solution Sonicated for $300 \mathrm{~s}$ (s96r000511 Sample)

B.5 Particle Size Distribution of SY-102 Sample in 0.1 M NaNO3 Solution Sonicated for $300 \mathrm{~s}(\mathrm{~s} 96 \mathrm{r} 000511$ Sample).- Rerun $\ldots \ldots \ldots \ldots \ldots \ldots \ldots \ldots \ldots \ldots$

B.6 Particle Size Distribution of SY-102 Sample in $1 \mathrm{M}$ NaNO3 Solution (s96r000511 Sample)

B.7 Particle Size Distribution of SY-102 Sample in $1 \mathrm{M}$ NaNO3 Solution Sonicated for $300 \mathrm{~s}$ (s96r000511 Sample) 
B.8 Particle Size Distribution of SY-102 Sample in DI Water (Vial A Sample-Run \#1) $\quad \ldots$. . B.8

B.9 Particle Size Distribution of SY-102 Sample in DI Water (Vial A Sample - Run \#2) . . . B.10

B.10 Particle Size Distribution of SY-102 Sample in DI Water (Vial A Sample - Run \#3) . . . B.12

B.11 Particle Size Distribution of SY-102 Sample in DI Water

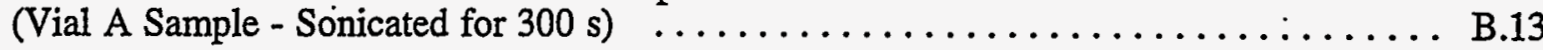

B.12 Particle Size Distribution of SY-102 Sample in DI Water (Vial B Sample) ... . . . . B.14

B.13 Particle Size Distribution of SY-102 Sample in DI Water (Vial B Sample - Sonicated for $300 \mathrm{~s}$ ) . . . . . . . . . . . . . . . . . . . .

B.14 Particle Size Distribution of SY-102 Sample in DI Water (Vial C Sample) . . . . . . B.16

B.15 Particle Size Distribution of SY-102 Sample in DI Water

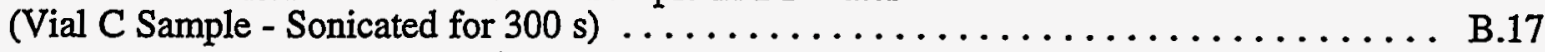

B.16 Particle Size Distribution of SY-102 Sample in DI Water (Vial D Sample) . . . . . . . B.18

B.17 Particle Size Distribution of SY-102 Sample in DI Water

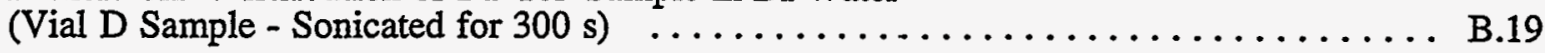

B.18 Particle Size Distribution of SY-102 Sample in DI Water

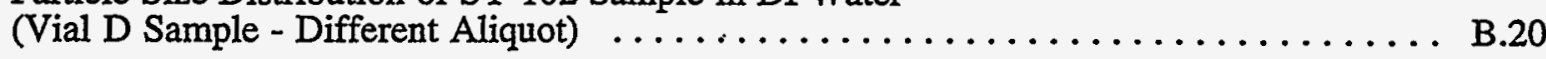

B.19 Particle Size Distribution of SY-102 Sample in DI Water (Vial E Sample) . . . . . . . B.21

B.20 Particle Size Distribution of SY-102 Sample in DI Water (Vial E Sample -

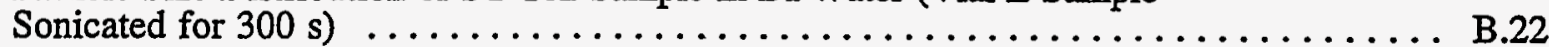

C.1 Predicted Velocity $(\mathrm{m} / \mathrm{s})$ and Distribution of Solid 1 Volume Fraction on Vertical Plane 21 ( 9 o'clock Position) at 15 Simulation Minutes for Coarser

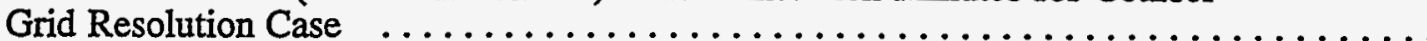

C.2 Predicted Velocity $(\mathrm{m} / \mathrm{s})$ and Distribution of Solid 5 Volume Fraction on Vertical Plane 21 (9 o'clock Position) at 15 Simulation Minutes for Coarser Grid

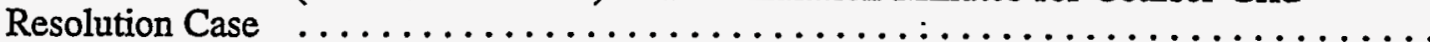

C.3 Predicted Velocity $(\mathrm{m} / \mathrm{s})$ and Distribution of Solid 7 Volume Fraction on Vertical Plane 21 ( 9 o'clock position) at 15 Simulation Minutes for Coarser Grid Resolution Case

C.4 Predicted Velocity $(\mathrm{m} / \mathrm{s})$ and Distribution of Solid.1 Volume Fraction on Vertical Plane 41 ( 9 o'clock Position) at 15 Simulation Minutes for Finer Grid Resolution Case

C.5 Predicted Velocity $(\mathrm{m} / \mathrm{s})$ and Distribution of Solid 5 Volume Fraction on Vertical Plane 41 ( 9 o'clock Position) at 15 Simulation Minutes for Finer Grid Resolution Case

C.6 Predicted Velocity $(\mathrm{m} / \mathrm{s})$ and Distribution of Solid 7 Volume Fraction on Vertical Plane ( 9 o'clock Position) at 15 Simulation Minutes for Finer Grid Resolution Case 
C.7 Predicted Velocity ( $\mathrm{m} / \mathrm{s})$ and Horizontal Distribution of Solid 7 Volume Fraction on Tank Bottom at 15 Simulation Minutes for the Coarser Grid Resolution Case .....

C.8 Predicted Velocity $(\mathrm{m} / \mathrm{s})$ and Horizontal Distribution of Solid 7 Volume Fraction at $43 \mathrm{~cm}$ (17 inches) (Height of the Jet Injection Nozzles) above Tank Bottom at 15 Simulation Minutes for the Coarser Grid Resolution Case $\ldots \ldots \ldots \ldots \ldots \ldots$

C.9 Predicted Velocity $(\mathrm{m} / \mathrm{s})$ and Horizontal Distribution of Solid 7 Volume Fraction on Tank Waste Surface ( $3.5 \mathrm{~m}$ or 138 inches above the Tank Bottom) at 15 Simulation Minutes for the Coarser Grid Resolution Case ......................

C.10 Predicted Velocity ( $\mathrm{m} / \mathrm{s})$ and Horizontal Distribution of Solid 7 Volume Fraction on Tank Bottom at 15 Simulation Minutes for Finer Grid Resolution Case . . . . . . . .

C.11 Predicted Velocity $(\mathrm{m} / \mathrm{s})$ and Horizontal Distribution of Solid 7 Volume Fraction at $43 \mathrm{~cm}$ above Tank Bottom at 15 Simulation Minutes for Finer Grid Resolution Case

C.12 Predicted Velocity $(\mathrm{m} / \mathrm{s})$ and Horizontal Distribution of Solid 7 Volume Fraction on Tank Waste Surface at 15 Simulation Minutes for Finer Grid Resolution Case .....

C.13 Predicted Velocity $(\mathrm{m} / \mathrm{s})$ and Distribution of Solid 1 Volume Fraction on Vertical Plane 21 (9:00 o'clock Position) at Two Simulation Hours for Case $1 \ldots \ldots \ldots \ldots$

C.14 Predicted Velocity $(\mathrm{m} / \mathrm{s})$ and Distribution of Solid 1 Volume Fraction on Vertical Plane 15 (11 o'clock Position) at Two Simulation Hours for Case 1

C.15 Predicted Velocity $(\mathrm{m} / \mathrm{s})$ and Distribution of Solid 5 Volume Fraction on Vertical Plane 21 (9 o'clock Position) at Two Simulation Hours for Case $1 \ldots \ldots \ldots \ldots \ldots$

C.16 Predicted Velocity $(\mathrm{m} / \mathrm{s})$ and Distribution of Solid 5 Volume Fraction on Vertical Plane 15 (11 o'clock Position) at Two Simulation Hours for Case $1 \ldots \ldots \ldots \ldots$

C.17 Predicted Velocity $(\mathrm{m} / \mathrm{s})$ and Distribution of Solid 7 Volume Fraction on Vertical Plane 21 (9 o'clock Position) at Two Simulation Hours for Case $1 \ldots \ldots \ldots \ldots \ldots$

C.18 Predicted Velocity $(\mathrm{m} / \mathrm{s})$ and Distribution of Solid 7 Volume Fraction on Vertical Plane 15 (11 o'clock Position) at Two Simulation Hours for Case $1 \ldots \ldots \ldots \ldots$. . .

C.19 Predicted Velocity $(\mathrm{m} / \mathrm{s})$ and Horizontal Distribution of Solid 1 Volume Fraction on the Tank Bottom at Two Simulation Hours for Case 1

C.20 Predicted Velocity $(\mathrm{m} / \mathrm{s})$ and Horizontal Distribution of Solid 1 Volume Fraction at $43 \mathrm{~cm}$ (17 inches) (Height of the Jet Injection Nozzles) above Tank Bottom at Two Simulation Hours for Case 1

C.21 Predicted Velocity $(\mathrm{m} / \mathrm{s})$ and Horizontal Distribution of Solid 1 Volume Fraction on the Tank Waste Surface (3.5 m or 138 inches above the Tank Bottom) at Two Simulation Hours for Case 1

C.22 Predicted Velocity $(\mathrm{m} / \mathrm{s})$ and Horizontal Distribution of Solid 5 Volume Fraction on the Tank Bottom at Two Simulation Hours for Case 1

C.23 Predicted Velocity $(\mathrm{m} / \mathrm{s})$ and Horizontal Distribution of Solid 5 Volume Fraction at $43 \mathrm{~cm}$ (17 inches) (Height of the Jet Injection Nozzles) above Tank Bottom at Two Simulation Hours for Case 1 
C.24 Predicted Velocity $(\mathrm{m} / \mathrm{s})$ and Horizontal Distribution of Solid 5 Volume Fraction on the Tank Waste Surface ( $3.5 \mathrm{~m}$ or 138 inches above the Tank Bottom) at

Two Simulation Hours for Case 1

C.25 Predicted Velocity $(\mathrm{m} / \mathrm{s})$ and Horizontal Distribution of Solid 7 Volume Fraction on the Tank Bottom at Two Simulation Hours for Case $1 \ldots \ldots \ldots \ldots \ldots \ldots$

C.26 Predicted Velocity $(\mathrm{m} / \mathrm{s})$ and Horizontal Distribution of Solid 7 Volume Fraction at $43 \mathrm{~cm}$ (17 inches) (height of the Jet Injection Nozzles) above Tank Bottom at

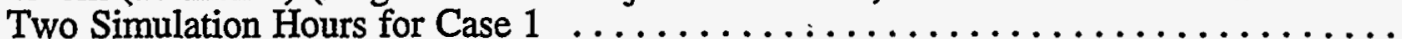

C.27 Predicted Velocity $(\mathrm{m} / \mathrm{s})$ and Horizontal Distribution of Solid 7 Volume Fraction on the Tank Waste Surface ( $3.5 \mathrm{~m}$ or 138 inches above the Tank Bottom) at

Two Simulation Hours for Case 1

C.28 Predicted Horizontal Distribution of Solid 1 Volume Fraction on the Tank Bottom at Two Simulation Hours for Case 1

C.29 Predicted Horizontal Distribution of Solid 2 Volume Fraction on the Tank Bottom at Two Simulation Hours for Case 1

C.30 Predicted Horizontal Distribution of Solid 3 Volume Fraction on the Tank Bottom at Two Simulation Hours for Case 1

C.31 Predicted Horizontal Distribution of Solid 4 Volume Fraction on the Tank Bottom at Two Simulation Hours for Case 1

C.32 Predicted Horizontal Distribution of Solid 5 Volume Fraction on the Tank Bottom at Two Simulation Hours for Case $1 \ldots \ldots \ldots \ldots \ldots \ldots$

C.33 Predicted Horizontal Distribution of Solid 6 Volume Fraction on the Tank Bottom at Two Simulation Hours for Case 1

C.34 Predicted Horizontal Distribution of Solid 7 Volume Fraction on the Tank Bottom at Two Simulation Hours for Case 1

C.35 Predicted Velocity Distribution on Vertical Plane 27 (7:30 position) at 55.8 Simulation Minutes for Case 1

C.36 Predicted Velocity Distribution on the Tank Bottom at Two Simulation Hours at

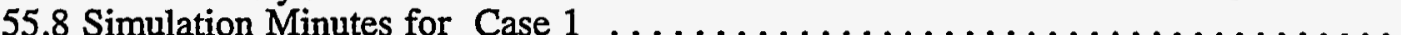

C.37 Estimated Tank Bottom Areas for Possible Sludge Mobilization at Different Velocity and Yield Stress Values for Case 1

C.38 Predicted Velocity $(\mathrm{m} / \mathrm{s})$ and Distribution of Solid 1 Volume Fraction on Vertical Plane 21 (9 o'clock Position) at Two Simulation Hours for Case $2 \ldots \ldots \ldots \ldots \ldots$. . . .

C.39 Predicted Velocity $(\mathrm{m} / \mathrm{s})$ and Distribution of Solid 5 Volume Fraction on Vertical Plane 21 (9 o'clock Position) at Two Simulation Hours for Case $2 \ldots \ldots \ldots \ldots \ldots$

C.40 Predicted Velocity $(\mathrm{m} / \mathrm{s})$ and Distribution of Solid 7 Volume Fraction on Vertical Plane 21 (9 o'clock Position) at Two Simulation Hours for Case 2

C.41 Predicted Velocity $(\mathrm{m} / \mathrm{s})$ and Horizontal Distribution of Solid 1 Volume Fraction on the Tank Bottom at Two Simulation Hours for Case 2 
C.42 Predicted Velocity $(\mathrm{m} / \mathrm{s})$ and Horizontal Distribution of Solid 5 Volume Fraction on the Tank Bottom at Two Simulation Hours for Case $2 \ldots \ldots \ldots \ldots \ldots \ldots$

C.43 Predicted Velocity $(\mathrm{m} / \mathrm{s})$ and Horizontal Distribution of Solid 7 Volume Fraction on the Tank Bottom at Two Simulation Hours for Case $2 \ldots \ldots \ldots \ldots \ldots \ldots$

C.44 Predicted Velocity $(\mathrm{m} / \mathrm{s})$ and Distribution of Solid 1 Volume Fraction on Vertical Plane 21 (9 o'clock Position) at Two Simulation Hours for Case $3 \ldots \ldots \ldots \ldots$. . . .

C.45 Predicted Velocity $(\mathrm{m} / \mathrm{s})$ and Horizontal Distribution of Solid 1 Volume Fraction on the Tank Bottom at Two Simulation Hours for Case 3

C.46 Predicted Velocity $(\mathrm{m} / \mathrm{s})$ and Distribution of Solid 5 Volume Fraction on Vertical Plane 21 (9 o'clock Position) at Two Simulation Hours for Case 3 .............

C.47 Predicted Velocity $(\mathrm{m} / \mathrm{s})$ and Horizontal Distribution of Solid 5 Volume Fraction on the Tank Bottom at two Simulation Hours for Case $3 \ldots \ldots \ldots \ldots \ldots \ldots$

C.48 Predicted Velocity $(\mathrm{m} / \mathrm{s})$ and Distribution of Solid 7 Volume Fraction on Vertical Plane 21 (9 o'clock Position) at Two Simulation Hours for Case $3 \ldots \ldots \ldots \ldots \ldots$. . . .

C.49 Predicted Velocity $(\mathrm{m} / \mathrm{s})$ and Horizontal Distribition of Solid 7 Volume Fraction on the Tank Bottom at Two Simulation Hours for Case $3 . \ldots \ldots \ldots \ldots \ldots \ldots$

C.50 Predicted Velocity $(\mathrm{m} / \mathrm{s})$ and Distribution of Solid 1 Volume Fraction on Vertical Plane 21 ( 9 o'clock Position) at Two Simulation Hours for Case $4 \ldots \ldots \ldots \ldots \ldots$. . . .

C.51 Predicted Velocity $(\mathrm{m} / \mathrm{s})$ and Horizontal Distribution of Solid 1 Volume Fraction on the Tank Bottom at Two Simulation Hours for Case $4 \ldots \ldots \ldots \ldots \ldots \ldots \ldots$

C.52 Predicted Velocity $(\mathrm{m} / \mathrm{s})$ and Distribution of Solid 5 Volume Fraction on Vertical Plane 21 (9 o'clock Position) at Two Simulation Hours for Case $4 \ldots \ldots \ldots \ldots \ldots$.

C.53 Predicted Velocity $(\mathrm{m} / \mathrm{s})$ and Horizontal Distribution of Solid 5 Volume Fraction on the Tank Bottom at Two Simulation Hours for Case $4 \ldots \ldots \ldots \ldots \ldots \ldots \ldots$

C.54 Predicted Velocity $(\mathrm{m} / \mathrm{s})$ and Distribution of Solid 7 Volume Fraction on Vertical Plane 21 (9 o'clock Position) at Two Simulation Hours for Case $4 \ldots \ldots \ldots \ldots \ldots$. .

C.55 Predicted Velocity $(\mathrm{m} / \mathrm{s})$ and Horizontal Distribution of Solid 7 Volume Fraction on the Tank Bottom at Two Simulation Hours for Case $4 \ldots \ldots \ldots \ldots \ldots \ldots$

C.56 Predicted Velocity $(\mathrm{m} / \mathrm{s})$ and Distribution of Solid 1 Volume Fraction on Vertical Plane 21 (9 o'clock Position) at Two Simulation Hours for Case 5 .............

C.57 Predicted Velocity $(\mathrm{m} / \mathrm{s})$ and Horizontal Distribution of Solid 1 Volume Fraction on the Tank Bottom at Two Simulation Hours for Case $5 \ldots \ldots \ldots \ldots \ldots \ldots$

C.58 Predicted Velocity $(\mathrm{m} / \mathrm{s})$ and Distribution of Solid 5 Volume Fraction on Vertical Plane 21 (9 o'clock Position) at Two Simulation Hours for Case 5 .............

C.59 Predicted Velocity $(\mathrm{m} / \mathrm{s})$ and Horizontal Distribution of Solid 5 Volume FractioN on the Tank Bottom at Two Simulation Hours for Case 5

C.60 Predicted Velocity $(\mathrm{m} / \mathrm{s})$ and Distribution of Solid 7 Volume Fraction on Vertical Plane 21 (9 o'clock Position) at Two Simulation Hours for Case 5 
C.61 Predicted Velocity (m/s) and Horizontal Distribution of Solid 7 Volume Fraction on the Tank Bottom at Two Simulation Hours for Case $5 . \ldots \ldots \ldots \ldots \ldots \ldots$

C.62 Predicted Velocity $(\mathrm{m} / \mathrm{s})$ and Distribution of Solid 1 Volume Fraction on Vertical Plane 21 (9 o'clock Position) at Two Simulation Hours for Case $6 \ldots \ldots \ldots \ldots \ldots$

C.63 Predicted Velocity $(\mathrm{m} / \mathrm{s})$ and Horizontal Distribution of Solid 1 Volume Fraction on the Tank Bottom at Two Simulation Hours for Case $6 \ldots \ldots \ldots \ldots \ldots \ldots$

C.64 Predicted Velocity $(\mathrm{m} / \mathrm{s})$ and Distribution of Solid 5 Volume Fraction on Vertical Plane 21 (9 o'clock Position) at Two Simulation Hours for Case 6 . . . . . . . . . . .

C.65 Predicted Velocity $(\mathrm{m} / \mathrm{s})$ and Horizontal Distribution of Solid 5 Volume Fraction on the Tank Bottom at Two Simulation Hours for Case 6 ................

C.66 Predicted Velocity $(\mathrm{m} / \mathrm{s})$ and Distribution of Solid 7 Volume Fraction on Vertical Plane 21 (9 o'clock Position) at Two Simulation Hours for Case $6 \ldots \ldots \ldots \ldots$. . .

C.66

C.67 Predicted Velocity $(\mathrm{m} / \mathrm{s})$ and Horizontal Distribution of Solid 7 Volume Fraction on the Tank Bottom at Two Simulation Hours for Case $6 \ldots \ldots \ldots \ldots \ldots$

C.68 Predicted Velocity $(\mathrm{m} / \mathrm{s})$ and Distribution of Solid 7 Volume Fraction on Vertical

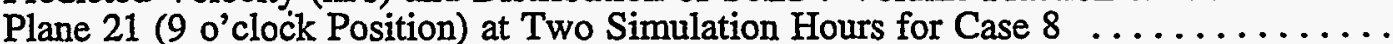

C.69 Predicted Velocity $(\mathrm{m} / \mathrm{s})$ and Horizontal Distribution of Solid 7 Volume Fraction on the Tank Bottom at Two Simulation Hours for Case $8 \ldots \ldots \ldots \ldots \ldots \ldots \ldots$

C.70 Predicted Velocity $(\mathrm{m} / \mathrm{s})$ and Distribution of Solid 7 Volume Fraction on Vertical Plane 21 (9 o'clock Position) at Two Simulation Hours for Case $9 \ldots \ldots \ldots \ldots \ldots$

C.70

C.71 Predicted Velocity $(\mathrm{m} / \mathrm{s})$ and Horizontal Distribution of Solid 7 Volume Fraction on the Tank Bottom at Two Simulation Hours for Case $9 \ldots \ldots \ldots \ldots \ldots$

C.71

C.72 Predicted Velocity $(\mathrm{m} / \mathrm{s})$ and Distribution of Solid 1 Volume Fraction on Vertical Plane 21 (9' o'clock Position) at Two Simulation Hours for Case 7 . . . . . . . . . .

C.73 Predicted Velocity $(\mathrm{m} / \mathrm{s})$ and Distribution of Solid 1 Volume Fraction on Vertical Plane 21 (9 o'clock Position) at Two Simulation Hours for Case 8 ............

C.74 Predicted Velocity $(\mathrm{m} / \mathrm{s})$ and Distribution of Solid 1 Volume Fraction on Vertical Plane 21 (9 o'clock position) at Two Simulation Hours for Case $10 \ldots \ldots \ldots \ldots$. .

C.75 Predicted Velocity $(\dot{\mathrm{m}} / \mathrm{s})$ and Distribution of Solid 7 Volume Fraction on Vertical Plane 21 (9 o'clock Position) at Two Simulation Hours for Case 7 . . . . . . . . .

C.76 Predicted Velocity $(\mathrm{m} / \mathrm{s})$ and Horizontal Distribution of Solid 7 Volume Fraction on the Tank Bottom at Two Simulation Hours for Case 7 ..................

C.77 Predicted Velocity $(\mathrm{m} / \mathrm{s})$ and Distribution of Solid 7 Volume Fraction on Vertical Plane 21 (9 o'clock Position) at Two Simulation Hours for Case $10 \ldots \ldots \ldots \ldots$. . . .

C.78 Predicted Velocity $(\mathrm{m} / \mathrm{s})$ and Horizontal Distribution of Solid 7 Volume Fraction on the Tank Bottom at Two Simulation Hours for Case $10 \ldots \ldots \ldots \ldots \ldots$. . . . . .

C.79 Predicted Velocity Contours of $0.2,0.5,1.0,1.5$, and $2.0 \mathrm{~m} / \mathrm{s}$ on Vertical Plane 21 ( 9 o'clock Position) at Two Simulation Hours for Case 7 (45 ft/s jets) 
C.80 Predicted Velocity Contours of $0.2,0.5,1.0,1.5$, and $2.0 \mathrm{~m} / \mathrm{s}$ on Vertical Plane 21 (9 o'clock Position) at Two Simulation Hours for Case $8(30 \mathrm{ft} / \mathrm{s}$ jets $) \ldots \ldots \ldots \ldots$ C. . . .

C.81 Predicted Velocity Contours of $0.2,0.5,1.0,1.5$, and $2.0 \mathrm{~m} / \mathrm{s}$ on Vertical Plane 21 (9 o'clock Position) at Two Simulation Hours for Case $10(15 \mathrm{ft} / \mathrm{s}$ jets $) \ldots \ldots \ldots \ldots$

C.82 Estimated Tank Bottom Areas for Possible Sludge Mobilization at.Different Velocity

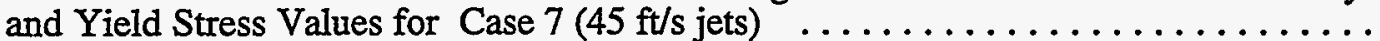

C.83 Estimated Tank Bottom Areas for Possible Sludge Mobilization at Different Velocity and Yield Stress Values for Case 8 and $9(30 \mathrm{ft} / \mathrm{s}$ jets $) \ldots$

C.84 Estimated Tank Bottom Areas for Possible Sludge Mobilization at Different Velocity and Yield Stress Values for Case 10 (15 ft/s jets) 


\section{Tables}

2.1 Results of Particle Size Analysis Under Different Solvent Conditions $\ldots \ldots \ldots \ldots$

3.1 Particle Size Distributions of Tank SY-102 Sludge $\ldots \ldots \ldots \ldots \ldots \ldots \ldots \ldots \ldots \ldots$

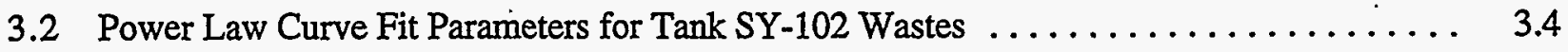

3.3 Maximum Variations (\%) of Predicted Volume Fractions over the Entire Tank in

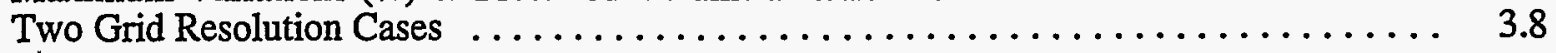

3.4 Assigned Initial Volume Fractions in the Sludge Layers $\ldots \ldots \ldots \ldots \ldots \ldots \ldots$

3.5 Maximum Variations (\%) of Predicted Volume Fractions over Entire Tank

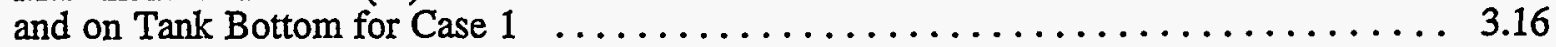

3.6 Maximum Volume Fractions and Segregation Factors on Tank Bottom for Case $2 \ldots \ldots$

3.7 Initial Volume Fractions of Seven Solid Size Fractions . . . . . . . . . . . . . 3.19

3.8 Predicted Maximum Variations (\%) of Predicted Volume Fractions over Entire Tank and on Tank Bottom at Two Simulation Hours for Case 2 . . . . . . . . 3.19

3.9 Comparison of the Predicted Maximum Volume Fractions over Entire Tank at Two Simulation Hours for Cases 1 and $2 \ldots \ldots \ldots \ldots \ldots \ldots \ldots \ldots$

3.10 Comparison of Predicted Maximum Variations of Predicted Volume Fractions over Entire Tank and on Tank Bottom at Two Simulation Hours for Cases 1,3 and $4 \ldots \ldots 3.21$

3.11 Comparison of the Predicted Maximum Volume Fractions on Tank Bottom

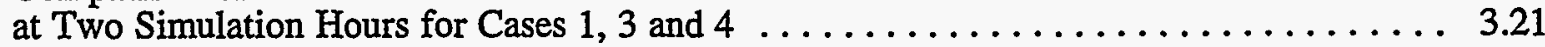

3.12 Comparison of Predicted Maximum Variations of Predicted Volume Fractions over Entire Tank and Tank Bottom at Two Simulation Hours, Cases 1, 5, and 6

3.13 Comparison of the Predicted Maximum Volume Fractions on Tank Bottom at Two Simulation Hours for Cases 1, 5, and 6

3.14 Comparison of Maximum Variations of Predicted Volume Fractions over Entire Tank and on Tank Bottom at Two Simulation Hours for Cases 1, 7, 8, 9, 10

3.15 Comparison of Predicted Maximum Volume Fractions on Tank Bottom at Two Simulation Hours for Cases $1,7,8,9$, and $10 \ldots \ldots \ldots \ldots \ldots \ldots \ldots \ldots \ldots \ldots \ldots \ldots$

4.1 Summary of Maximum Variations (\%) of Predicted Volume Fractions over Entire Tank after Two Simulation Hours for All Ten Cases ..............

4.2 Summary of Predicted Maximum Volume Fractions on Tank Bottom at Two Simulation Hours after Two Simulation Hours for All Ten Cases 



\subsection{Introduction}

Wastes stored in Hanford Tank 241-SY-102 are planned to be retrieved from that tank and transferred to 200 East Area through the new pipeline Replacement Cross Site Transfer System (RCSTS). Because the planned transfer of this waste will use the RCSTS, the slurry that results from the mobilization and retrieval operations must meet the applicable waste acceptance criteria for this system. This report describes results of the second phase (the detailed assessment) of the SY-102 waste retrieval study, which is a part of the efforts to establish a technical basis for mobilization of the slurry, waste retrieval, and slurry transport.

Hanford Tank 241-SY-102 is located in the SY Tank Farm in the Hanford Site's 200 West Area. It was built in 1977 to serve as a feed tank for 242-S Evaporator/Crystallizer, receiving supernatant liquid from S, SX, T, and U tank farms. Since 1981, the primary sources of waste have been from 200 West Area facilities, e.g., T-Plant decontamination operations, Plutonium Finishing Plant operations, and the 222-S Laboratory. It is the only active-service double-shell tank (DST) in the 200 West Area and is used as the staging tank for cross-site transfers to 200 East Area DSTs (DiCenso et al. 1995). The tank currently stores approximately $470 \mathrm{~kL}$ (125 kgal) of sludge wastes from a variety of sources including the Plutonium Finishing Plant, T-Plant, and the 222-S Laboratory (DiCenso et al. 1995). In addition to the sludge, approximately twice this amount (about $930 \mathrm{~kL}$ ) of dilute, noncomplexed waste forms a supernatant liquid layer above the sludge.

Under Phase I of the waste retrieval assessment study, we conducted the preliminary assessment to determine the potential for Tank SY-102 waste properties being adversely affected by mixing of the current tank contents or by the injection of additional diluent into the tank during sludge mobilization (Onishi and Hudson 1996). We determined potential chemical reactions with the equilibrium chemical reaction code, GMIN (Felmy 1990), and associated rheology changes to evaluate the feasibility of Tank SY-102 waste retrieval. We also examined approximate ranges of design and operational conditions and their potential impact on waste properties. The predicted transport behavior of the resulting slurries was.also evaluated for its acceptance for the RCSTS. The Phase 1 preliminary assessment indicated that mixing the tank sludge with two selected diluents and resulting waste heating are not expected to adversely impact waste properties for slurry transport in RCSTS and didn't turn up any problems with activities of mixing, diluting, heating, or transferring the waste of 102-SY; thus the waste pipeline transfer through RCSTS was feasible.

This Phase I conclusion was based on simplified assumptions and conditions, e.g., tank wastes are fully mixed; data in the Tank 241-SY-102 characterization report (DiCenso et al. 1995) are accurate; and formulas for fluid and slurry rheology are applicable. Most of the uncertainties on the preliminary assessment was related to data of waste characteristics and the assumption of the tank being fully mixed. To alleviate these uncertainties, the preliminary assessment report (Onishi and Hudson 1996) recommended: 1) additional lab analysis to determine solid phase speciation, 2) evaluate more closely the potential for chemical reactions and associated rheology changes by incorporating spatial and temporal distributions of the tank wastes, 3) further consideration of kinetic effects with waste movements, 4) review the source documents of the SY-102 Tank characterization report (DiCenso et al. 1995), 5) find better data on rheology, sedimentation, and particle size distributions, and 6) evaluate engineering correlations on slurry transport behavior.

The Phase II study addressed these uncertainties on Tank SY-102 waste retrieval by conducting the recommended studies listed above. Specifically it focused on 1) identifying solid phases of the SY-102 waste by using transmission electron microscopy (TEM); 2) conducting laboratory experiments to measure rheology, zeta potential to evaluate solid agglomeration, and sedimentation; 3) measuring particle size distributions of the wastes; and 4) conducting three-dimensional modeling to simulate the tank pump jet mixing and evaluating potential effects (if any) of waste mixing on equilibrium and kinetic chemical reactions and rheology changes. 
Section 2 discusses the experimental and measurement results, and Section 3 presents the tank mixing modeling results obtained by the three-dimensional flow-transport code, TEMPEST (Trent and Eyler 1993; Onishi et al. 1996). The summary and conclusions are presented in Section 4. References cited in the report are listed in Section 5. Appendix A presents electron microscopy results, and Appendix B describes particle size analysis results. Figures showing time-dependent, three-dimensional simulation results of the pump jet mixing are presented in Appendix C. 


\subsection{Rheological Measurements and Analysis}

Predictions of the behavior of Tank 241-SY-102 waste during retrieval depend strongly on the properties of the waste. As mentioned before, the properties of interest here are the rheological characteristics - that is, the relationship between amplitude and rate of deformation of the material and the stress field. This relationship exists through dynamic viscosity. For non-Newtonian fluids, - viscosity depends on the rate at which the material is deformed and the concentration of solid particles. For better predictive modeling, the knowledge of relation between viscosity, concentration, and shear rate is required.

As mentioned in the report by Onishi and Hudson (1996), a variety of precipitated insoluble solid species exist in Tank SY-102, many of which are expected to be colloidal. It has also been shown that colloidal agglomeration behavior can have significant impact on such macroscopic properties as slurry viscosity, yield strength, particle density, and sedimentation rates (LaFemina 1995a). Most of the experiments performed here were designed and performed to highlight the relationship between such macroscopic behavior and the microstructural features of the SY-102 waste.

\subsection{Experimental Procedure}

\subsubsection{Solid Phase Morphological Characterization}

The crystallinity, morphology, chemical composition, and crystalline phases of samples of Tank 241-SY-102 sludge were studied using transmission electron microscopy (TEM), electron energy dispersive spectroscopy (EDS), and electron diffraction. The details of the approach used is provided in Liu et al. (1995). In summary, the process involved dispersing a small quantity of the sampler $(\sim 1 \mathrm{mg})$ in a solution of methanol $(<5 \mathrm{ml})$ and placing a small drop of the solution on a TEM copper grid covered for electron microscopy analysis.

The morphology, distribution, and size of primary particles that compose the agglomerates were obtained from electron imaging. Chemical composition of the particles was identified by EDS. The structure of the crystals was obtained from electron diffraction patterns that were compared with the JCPDS-EDDTM database published by the JCPDS International Center for Diffraction Data.

\subsubsection{Particle Size Distribution}

Most of the particle size analysis methods selected will determine, within the measurement uncertainty of the instrument, the size distribution of the particles with no regard to the particular conditions under which size characterization was performed. Although this may be acceptable for non-colloidal or, in general, non-interacting particles, when characterizing colloids, it is important to test how the solution chemistry or shear (stress) field change the particle size distribution. The word particles is used rather casually here. A better term is aggregates, which comprised a large number of more elemental primary particles that can be as small as several nanometers, as will be shown from the TEM images in Section 2.2.1. For a more comprehensive discussion on parameters that affect aggregate size distribution, the reader is referred to LaFemina (1995b).

Particle size distributions were measured by the Microtrac full range analyzer (FRA) and photon correlation spectroscopy (Brookhaven Zetaplus). Samples for particle size were prepared in three conditions (if enough sample was available): distilled deionized water; $0.1 \mathrm{M} \mathrm{NaNO}_{3}$, and $1 \underline{\mathrm{M}}$ $\mathrm{NaNO}_{3}$. These conditions were chosen to encompass the range of electrolyte conditions that can affect flocculation and measured particle size. The particle sizes obtained are not primary particles; these measurements represent the size of the flocs or aggregates present in the waste as exists in the particle size analysis system. The primary particle size was determined by transmission electron 
microscopy, as mentioned in Section 2.1.1. In addition to variation in the solution chemistry, the effect of sonication (a mechanical means) on the particle size distribution was investigated.

\subsubsection{Zeta Potential}

Zeta potential is a measure of the surface charge density of particles. The relation between zeta potential and agglomeration has been shown (Rector and Bunker 1995). Zeta potential measurements were performed on dilute suspensions of the SY-102 waste in distilled deionized water using electrophoretic light scattering (Brookhaven Zetaplus). The $\mathrm{pH}$ was varied with nitric acid and sodium hydroxide.

\subsubsection{Rheology}

The rheology experiments were performed on a rotational viscometer, Haake CV-20 rheometer with a cone-and-plate sensor geometry. Sample volumes were approximately $0.20 \mathrm{~mL}$. Three sets of experiments were performed: controlled rate experiments, where the shear stress as a function of shear rate was determined (flow curve); a time-dependent viscosity experiments, where the changes in viscosity as a function of time for different shear rates were measured (viscosity-time curve); oscillatory experiments, where the amplitude of storage and loss terms of the complex shear modulus as a function of small amplitude oscillation frequency were measured.

Viscometry experiments were performed in the steady shear rate mode using the PK 45/4 cone and plate sensor. For flow curve determination, the shear rates were varied between 0 and $300 \mathrm{~s}^{-1}$. Three different fluids were tested: SY-102 stock, as received from 222-S laboratory, a 99 wt\% stock ( $1 \%$ by weight dilution with $1 \underline{\mathrm{M}} \mathrm{NaNO}_{3}$ solution), and a 52 wt\% stock ( $48 \%$ by weight dilution with $1 \underline{\mathrm{M}} \mathrm{NaNO}_{3}$ solution).

For time-dependent experiments, shear rates employed included $0.06,0.1,0.2,10$ and $100 \mathrm{~s}^{-1}$ for approximately 30 minutes continuously. For the latter two shear rates, shear on-shear off-shear on experiments were performed with 30 minute intervals. These experiments were performed to determine the time evolution of the viscosity under steady shear conditions, targeted to understand whether or not the material exhibits any rheopexy or thixotropy.

Dynamic oscillation experiments were performed with the same geometry over the frequency range 0.01 to $9.6 \mathrm{~Hz}$. The strain amplitude was $1 \%$.

\subsubsection{Sedimentation}

Samples of the SY-102 waste were placed in a test tube and subjected to centrifugation. The revolutions per minute (rpm) was converted to acceleration. The rpm was measured by a light spectrometer. Sediment height was measured by a calibrated test tube. The acceleration obtained is an average value since the acceleration varies in the test tube as a function of position.

\subsection{Results}

\subsubsection{TEM Characterization Results.}

A detailed synopsis of the TEM results is provided in Appendix A. The morphology, distribution, and sizes of particles were evaluated by electron imaging. The chemical composition of the particles was identified by EDS. And the crystal structures of the particles were studied by electron diffraction pattern. We present a short summary of the results in this section.

The morphology of the agglomerates is dominated by large size $(2-5 \mu \mathrm{m} \times 10-30 \mu \mathrm{m}) \mathrm{NaOH}$ rods surrounded by finer primary particles of various materials. A large population of amorphous 
silica and iron hydroxide primary particles was observed in the nanometer size range. Also, needlelike $\mathrm{FeO}(\mathrm{OH})$ agglomerates and sharp-edges plate-like $\gamma-\mathrm{Al}_{2} \mathrm{O}_{3}$ particles were found. Irregular $\mathrm{U}$ containing agglomerates and rectangular $\mathrm{Al} / \mathrm{Cr}$-containing particles were also present. The major crystalline phases observed include: micrometer-sized $\gamma-\mathrm{Al}_{2} \mathrm{O}_{3}$, needle-like $\mathrm{FeO}(\mathrm{OH})$ and $\mathrm{Al}_{2} \mathrm{Si}_{4} \mathrm{O}_{10}$. The minor crystalline phases observed were rod-like particles of hydroxylapatite, $\mathrm{Ca}_{5}(\mathrm{PO})_{3}(\mathrm{OH})$ and single crystalline gibbsite, $\mathrm{Al}(\mathrm{OH})_{3}$.

In the preliminary assessment study, we assumed the all $\mathrm{Al}$ solids to be gibbsite for the chemical reaction modeling (Onishi and Hudson 1996). The current TEM identified $\mathrm{Al}$ solids to be $\gamma-\mathrm{Al}_{2} \mathrm{O}_{3}$, $\mathrm{Al}_{2} \mathrm{Si}_{4} \mathrm{O}_{10}$, and gibbsite. Gibbsite and $\gamma-\mathrm{Al}_{2} \mathrm{O}_{3}$ are chemically similar. Moreover, existence of $\gamma-\mathrm{Al}_{2} \mathrm{O}_{3}$ would slightly increase the amount of water in the solution, as compared with gibbsite. Thus, the preliminary assessment with gibbsite may have slightly underestimated the amount of solids being dissolved, resulting in a slightly more conservative estimate (a worse case) for the retrieval operation. Note that the preliminary assessment also included cases of additional water being injected into the tank as an diluent. These additional-water cases also indicate that there are no adverse effects for waste retrieval operation as well. Although the current TEM also identified $\mathrm{Al}_{2} \mathrm{Si}_{4} \mathrm{O}_{10}$ as one of the solids present in the sludge, several measurements of a Si amount in the sludge indicate that the sludge has much smaller amount of Si than the amount of Al (DiCenso et al. 1995). Thus, a majority of $\mathrm{Al}$ is not expected to form a solid with Si. More over, $\mathrm{Al}_{2} \mathrm{Si}_{4} \mathrm{O}_{10}$ is very insoluble and has very long kinetic reaction time to form. The preliminary assessment has aqueous $\mathrm{Si}$ species to be $\mathrm{H}_{2} \mathrm{SiO}_{4}{ }^{-2}$ with only $0.007 \mathrm{~m}$, matching the measured amount of dissolved $\mathrm{Si}$ in the sludge very well(DiCenso et al. 1995). Thus the current TEM on Al supports the conclusion of the preliminary assessment with the selection of gibbsite as the dominating Al solid.

In the preliminary assessment, we also treated Fe solids in the sludge to be insoluble. The $\mathrm{Fe}$ solid, $\mathrm{FeO}(\mathrm{OH})$ identified by the TEM is very insoluble, thus, confirming the assumption of $\mathrm{Fe}$ solids in the preliminary assessment was valid. TEM also identified $\mathrm{NaOH}$ as one of the main solids in the sludge sample. The chemical modeling conducted in the preliminary assessment concluded that there is no $\mathrm{NaOH}$ solid. The $\mathrm{NaOH}$ rods shown in Appendix A was probable formed as a results of the drying of the TEM sample, rather than actually NaOH solids exist in the sludge in the tank.

The current TEM did not attempt to identify all solids in the sludge, including many expected solids. The preliminary assessment predicted that there are many other solids, e.g., Na solids of $\mathrm{NaNO}_{3}, \mathrm{NaF}$, thenardite and halite in the sludge.

As discussed above, the TEM characterization results indicted that the preliminary analysis performed by Onishi and Hudson (1996) is either accurate or is somewhat conservative, thus confirming its conclusion that the mixing of the sludge and supernate (with and without additional water as the diluent) and heating of the tank waste do not adversely impact the waste rheology for the retrieval operation.

\subsubsection{Particle Size Distribution}

Table 2.1 shows the particle sizes from the FRA in number-averaged $(m n)$ and volume-averaged $(m v)$ format. The probability distribution functions for particle sizes are presented in Appendix B. Sample A was analyzed by the PCS technique and the diameter obtained was $0.565 \mu \mathrm{m}$. In general, the PCS values should correspond to the FRA $m n$. The $m n$ for DI water conditions was $0.422 \pm 0.042$ $\mu \mathrm{m}$ and using the results from sample $A$ under these conditions, the variance of the measurement technique was calculated as 0.052 . Similarly, for the volume-averaged measurement, the DI water conditions yielded an average diameter of $6.435 \pm 1.025 \mu \mathrm{m}$ and a variance of the measurement technique of 1.770. Thus, the error in measuring different samples is smaller than what was obtained from 4 measurements of the same sample.

Examination of the FRA $m n$ and PCS effective diameters show that the system is colloidal. As a rule of thumb, particles below $1 \mu \mathrm{m}$ compose colloidal systems. The FRA volume-averaged sizes 
Table 2.1. Results of Particle Size Analysis Under Different Solvent Conditions

\begin{tabular}{|c|c|c|c|}
\hline $\begin{array}{l}\text { SY-102 Sample } \\
\text { Description }\end{array}$ & Solvent Conditions & $\begin{array}{c}\text { Volume-Averaged } \\
\text { Mean }\end{array}$ & $\begin{array}{l}\text { Number-Averaged } \\
\text { Mean }\end{array}$ \\
\hline \multirow[t]{5}{*}{ s96r000511 } & DI water & 6.043 & 0.39 \\
\hline & $0.1 \mathrm{M} \mathrm{NaNO}_{3}$ & 6.029 & 0.397 \\
\hline & $\begin{array}{l}0.1 \mathrm{M} \mathrm{NaNO}_{3} \\
\text { sonicated } 300 \mathrm{~s}\end{array}$ & 4.383 & 0.404 \\
\hline & $1 \mathrm{M} \mathrm{NaNO}_{3}$ & 7.245 & 0.866 \\
\hline & $\begin{array}{l}1 \mathrm{M} \mathrm{NaNO}_{3} \\
\text { sonicated } 300 \mathrm{~s}\end{array}$ & 3.305 & 0.753 \\
\hline Vial A & DI water & 9.322 & 0.488 \\
\hline Rerun & DI water & 6.093 & 0.491 \\
\hline Rerun & DI water & 8.38 & 0.411 \\
\hline \multirow[t]{2}{*}{ Rerun } & DI water & 5.668 & 0.389 \\
\hline & $\begin{array}{l}\text { DI water } \\
\text { sonicated } 300 \text { s }\end{array}$ & 4.637 & 0.374 \\
\hline \multirow[t]{2}{*}{ Vial B } & DI water & 5.954 & 0.396 \\
\hline & $\begin{array}{l}\text { DI water } \\
\text { sonicated } 300 \text { s. }\end{array}$ & 4.12 & 0.327 \\
\hline \multirow[t]{2}{*}{ Vial C } & DI water & 5.444 & 0.508 \\
\hline & $\begin{array}{l}\text { DI water } \\
\text { sonicated } 300 \text { s }\end{array}$ & 3.719 & 0.389 \\
\hline \multirow[t]{2}{*}{ Vial D } & DI water & 5.988 & 0.402 \\
\hline & $\begin{array}{l}\text { DI water } \\
\text { sonicated } 300 \text { s }\end{array}$ & 4.518 & 0.379 \\
\hline Vial D different aliquot & DI water. & 5.914 & 0.411 \\
\hline \multirow[t]{2}{*}{ Vial E } & DI water & 8.331 & 0.402 \\
\hline & $\begin{array}{l}\text { DI water } \\
\text { sonicated } 300 \text { s }\end{array}$ & 5.467 & 0.381 \\
\hline
\end{tabular}

show that some larger particles/aggregates are present. Since the larger particles/aggregates are weighted more in volume-averaging, it is understandable that the average size is greater than the FRA $m n$ and PCS values. Comparing the particle size results obtained from the s96r000511 sample (in the absence of sonication) shows that the increases in the electrolyte strength from 0.1 to $1 \mathrm{M} \mathrm{NaNO}_{3}$ result in a doubling of the number-averaged size and a slight increase in the volume-averaged size. Essentially, producing a strongly attractive system with the $1 \underline{M}$ electrolyte concentration results in more aggregation.

Since the colloidal analysis was done under the guise of obtaining insight into waste dislodging, it is instructive to review the effect of sonication. This consists of turning the Microtrac's sonicator to 
full power for 300 seconds. Unfortunately, the treatment is not readily described in terms of power input or some other readily definable quantity. Therefore, the results are taken as a qualitative indication of the ease of disrupting agglomerates. To describe the effect, the difference in number- and volume-averaged were calculated from successive runs. The successive runs were ambient conditions followed by the sonication. For the DI water conditions, the $\Delta m v=1.785 \pm 0.678 \mu \mathrm{m}$ and $\Delta m n=$ $0.12 \pm 0.15 \mu \mathrm{m}$. Thus, the sonication breaks up the largest agglomerates in the size distribution, which is more clearly evident on the figures in Appendix B. From the number-averaged inspection, there is no appreciable change in the system. Similar behavior was observed for the $0.1 \underline{M}$ and $1 \underline{M}$ conditions.

The most recent Tank Characterization Report by DiCenso et al. (1995) reported the distribution of particle sizes for a sample of Tank SY-102. It is clear that the particle size distributions presented in the current report are significantly smaller and more broad-band than the results in DiCenso.

Although a light-scattering technique was used in the work, the mean particle diameter based on population ranged between 10-20 $\mu \mathrm{m}$ and based on volume ranged between 50-60 $\mu \mathrm{m}$. These numbers are significantly different from what we found for this report.

Since the current particle size distribution results were not available until near the end of this study, the pump mixing simulation in this study was performed with the solid sizes varying from 10 to $175 \mu \mathrm{m}$, as reported in Dicenso et al (See Table 3.1 in Section 3). The use of these solid sizes places the analysis on the conservative side, since larger particles tend to settle faster. As discussed in detail in Section 3, even with this size distribution reported by DiCenso et al (1995), the current threedimensional SY-102 tank model predicted that the bulk of the sludge and supernate are almost completely mixed in the tank within a short period of tim (less than one to two hours). Thus, the assumption of the completely mixed wastes in the tank used for the preliminary assessment (Onishi and Hudson 1996) is supported by the current particle size distribution measurements and the modeling, as discussed in Section 3 in detail.

\subsubsection{Zeta Potential}

The zeta potential for the SY-102 waste sample as a function of $\mathrm{pH}$ is shown in Figure 2.1. The solid line shown on this figure is a curve fit into the data points, following a relationship $\zeta=\frac{300}{\mathrm{pH}^{1.65}}-44$. The isoelectric point is approximated to be 3.2. Parks (1965). has shown that the isoelectric points of metal oxide/hydroxide phases vary according to the binding force and correspondingly to the phase. Specifically, $\mathrm{RO}_{2}$ phases such as silica possess isoelectric points typically in the $\mathrm{pH}$ range of 2 to 4 . Clearly, the silica phase is controlling the surface charge development of the SY-102 waste as indicated by the zeta potential. This is consistent with the TEM results that show a presence of the colloidal amorphous silica in the aggregates.

\subsubsection{Rheology}

The SY-102 viscosity was measured as a function of time. Figure 2.2 is the result of the measurements for the shear rates of 0.06 and $0.2 \mathrm{~s}^{-1}$. The experiment was performed over a long enough time, exceeding 100 minutes, to allow the material to respond to the stress field. After the measurement at each shear rate was terminated, the sample was discarded and a new one was loaded. Although the procedure for loading was all the same, it is expected that the material behavior would show some variance due to sample loading. The most notable feature of the results in Figure 2.2 is that the viscosity increases as a function of time. This behavior is termed rheopexy or antithixotropy. Similar behavior was previously observed with the NCAW simulant (Chang and Smith 1996). The $0.1 \mathrm{~s}^{-1}$ test did not show this behavior. The difference in the rheological behavior is attributed to errors in sample loading. More specifically, the rheopectic nature of the system might have been perturbed during the loading of the sample for the $0.1 \mathrm{~s}^{-1}$ test. Inspection of the figure shows an incubation period before the onset of the rheopexy. To obtain rheopexy, the microstructure of the 


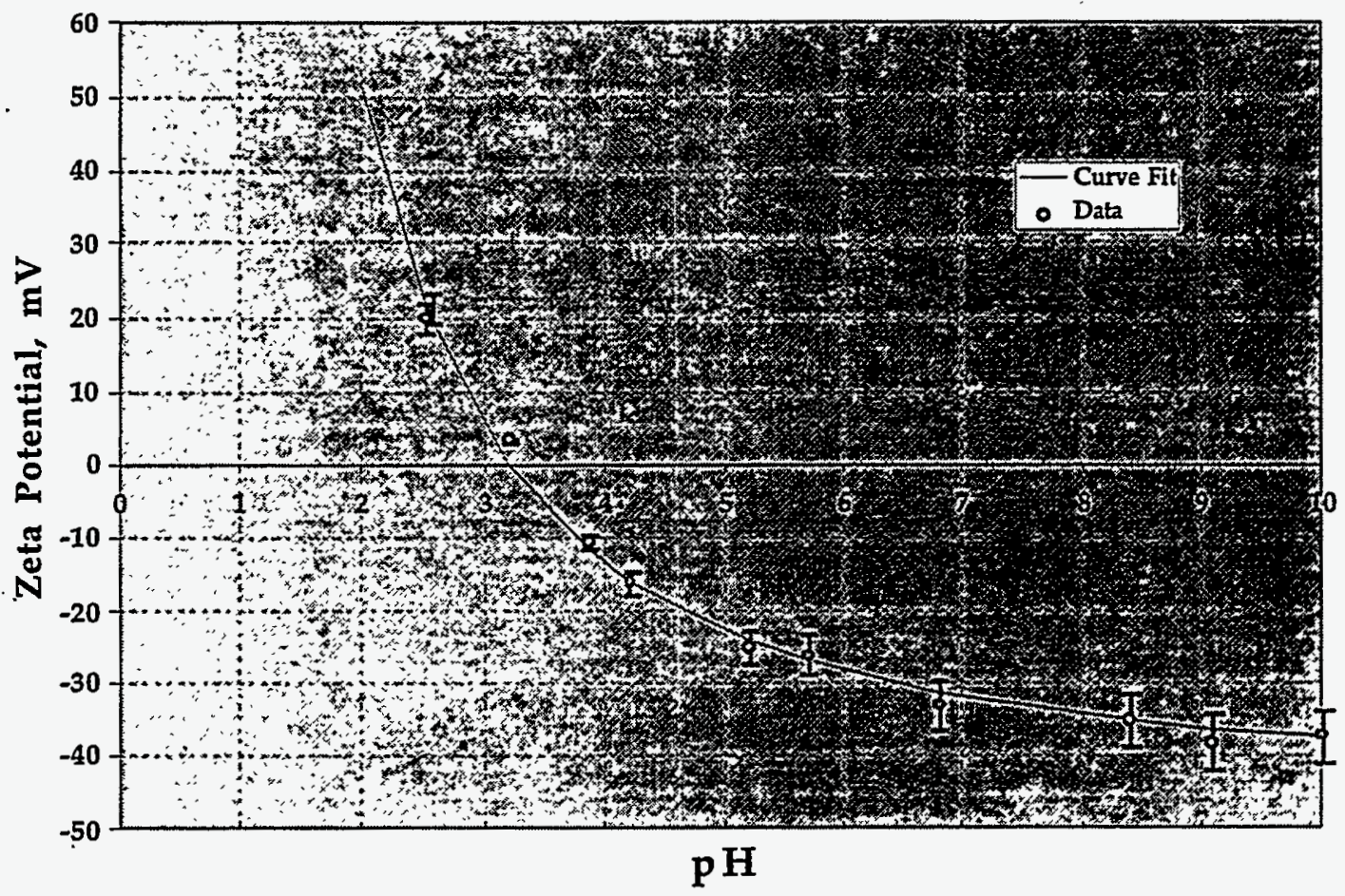

Figure 2.1. Zeta Potential as a Function of pH for Tank SY-102 Waste Slurry

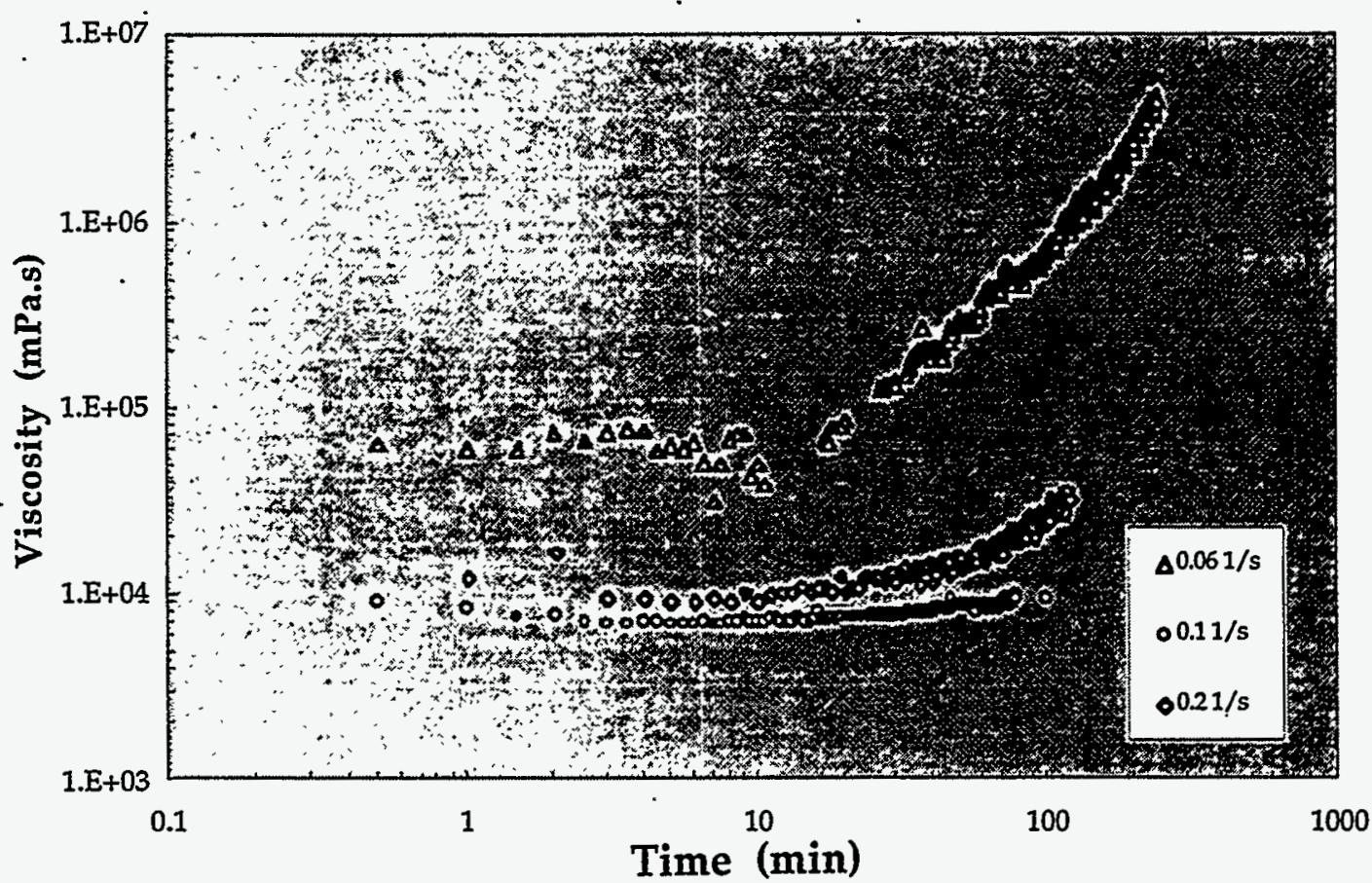

Figure 2.2. Rheopexy or Antithixotropic Behavior of Tank SY-102 Waste at Low Shear Rates 
suspension must be altered so that particles are aggregated. So the incubation is a result of the time required for particle collisions to be enacted by the small applied strain rates. That is, at the low. shear rate limit, and in the absence of large shearing stresses, the particle collision rate is increased and agglomeration is promoted. It has been shown that the agglomeration results in higher viscosities in colloidal suspensions (Rector and Bunker). Note that at $0.06 \mathrm{~s}^{-1}$ results show a two order-ofmagnitude jump in the viscosity of the slurry, and after approximately 3 hours, the viscosity exceeds $\sim 5 \times 106$ mPa-s.

Figure 2.3 presents the results of tests in the shear on-shear off-shear on mode for strain rates of 10 and $100 \mathrm{~s}^{-1}$. In the $10 \mathrm{~s}^{-1}$ test, the suspension shows thixotropic behavior. The aggregates present in the starting material or induced by shear are broken up. Examination of the initial curve at the terminal region and the data produced after the $30 \mathrm{~min}$ static period shows that the structure did not rebuild. The difference in the curves suggests that the system behaves thixotropically in this region and an area estimate of thixotropy $(8029 \mathrm{mPa}-\mathrm{s})$ was obtained. At $100 \mathrm{~s}^{-1}$, the aggregates in the suspension are not destroyed any further, and time effects are absent. It is expected that the sizes of the aggregates have reached some equilibrium value controlled more by the chemistry than the stress field.

Figures 2.2 and 2.3 present a very important set of results. These results suggest that if the slurry is moving at a slow enough rate, aggregation of the primary particles and smaller aggregates to form larger aggregates is promoted. In fact, the continuous increase in the viscosity at the lowest shear rate

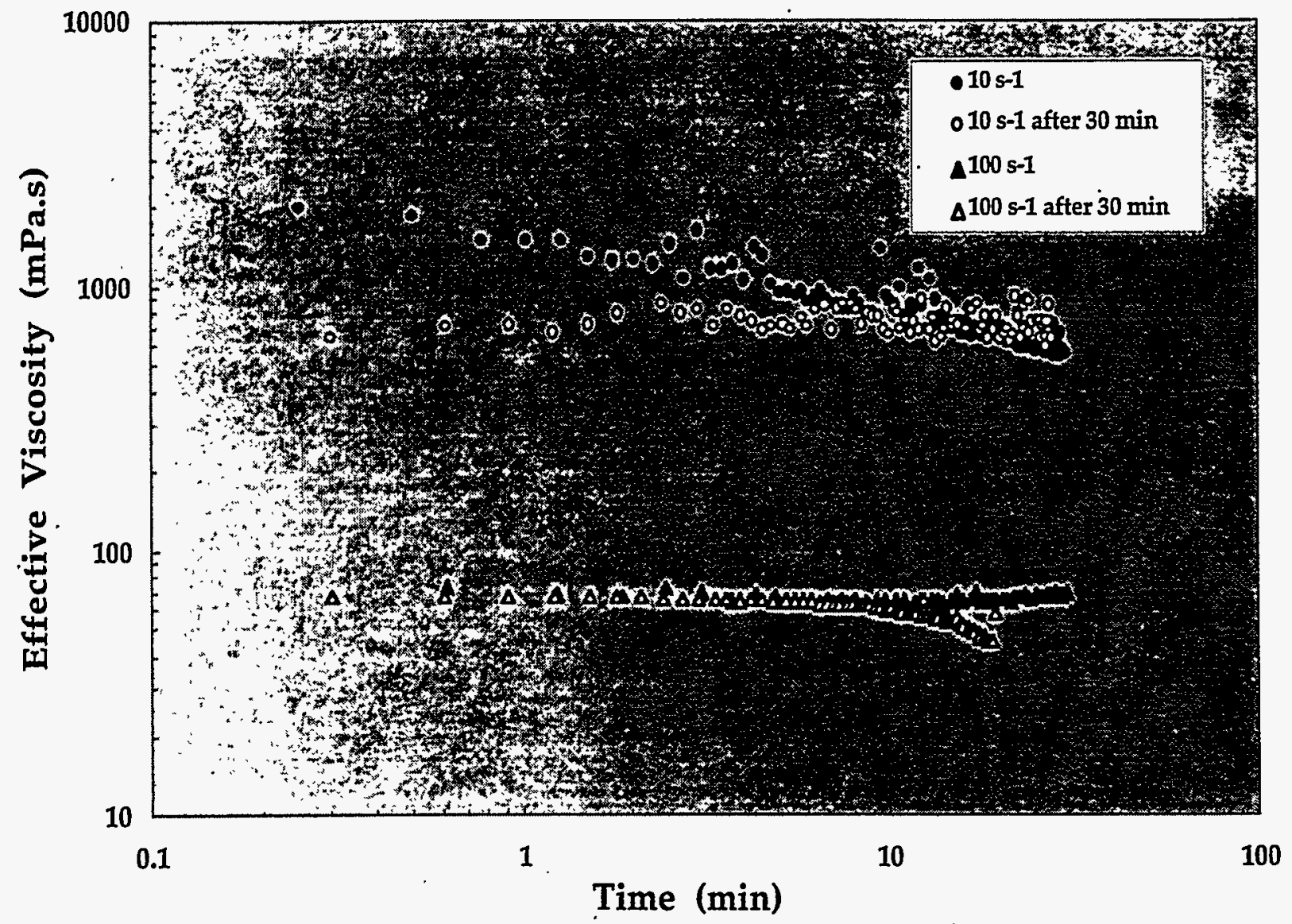

Figure 2.3. Thixotropic Behavior of Tank SY-102 Waste at High Shear Rates 
suggests that gelation may be possible. As the fluid is sheared faster, the rate of deaggregation due to higher shearing stresses increases and balances the rate of agglomeration at shear rate $\sim O\left(1 \mathrm{~s}^{-1}\right)$. At a shear rate of $10 . \mathrm{s}^{-1}$, the deaggregation rate exceeds the agglomeration rate and as a result a thixotropic behavior is observed. And finally, at a high enough rate, the agglomerates have reached an equilibrium size distribution, mainly controlled by the chemistry. Gelatin may thus be avoided by operating at higher shear rates than $1 \mathrm{~s}-1$. Note in Figure 2.2 that if the low shear rate of $0.061 / 5$ is not maintained more than 10 minutes, rheopexy is not measurable. Since the rotation period of the jets in SY-102 is two minutes, we anticipate such rheopexy not to occur.

As will be discussed in Section 3, we used the sludge viscosity to be $12 \mathrm{~Pa}$-s for a base case of the pump jet mixing simulations. This selection was based on these viscosity measurements (See Figures 2.2 and 2.3) and an initial model prediction that the strain rate is of the orders of $1-10 \mathrm{~s}^{-1}$ around the edge of the jets (near the tank wall) along the jet center. We also used the sludge viscosity of $4,500 \mathrm{~Pa}-\mathrm{s}$ to represent high viscosity conditions for the modeling. The model then internally calculated the viscosity changing with solid concentrations as a part of the simulation.

Rheograms were produced for the SY-102 stock suspension, 99 wt\% stock (diluted with $1 \underline{M}$ $\mathrm{NaNO}_{3}$ ) and $52 \mathrm{wt} \%$ stock, as shown in Figure 2.4: The variance in the data seems to be highest in the $99 \mathrm{wt} \%$ stock. The spikes in the.rheogram were attributed to two-phase flow anomalies, possibly due to phase segregation or shear-induced migration near the sensor wall. The undiluted solution has a classic behavior which may be represented as a Bingham Plastic fluid over the range of 0 to $300 \mathrm{~s}^{-1}$ shear rates.(a) The constitutive rheological model of this fluid can be presented as

$$
\tau=2.7 \mathrm{~Pa}+0.005 \dot{\gamma}
$$

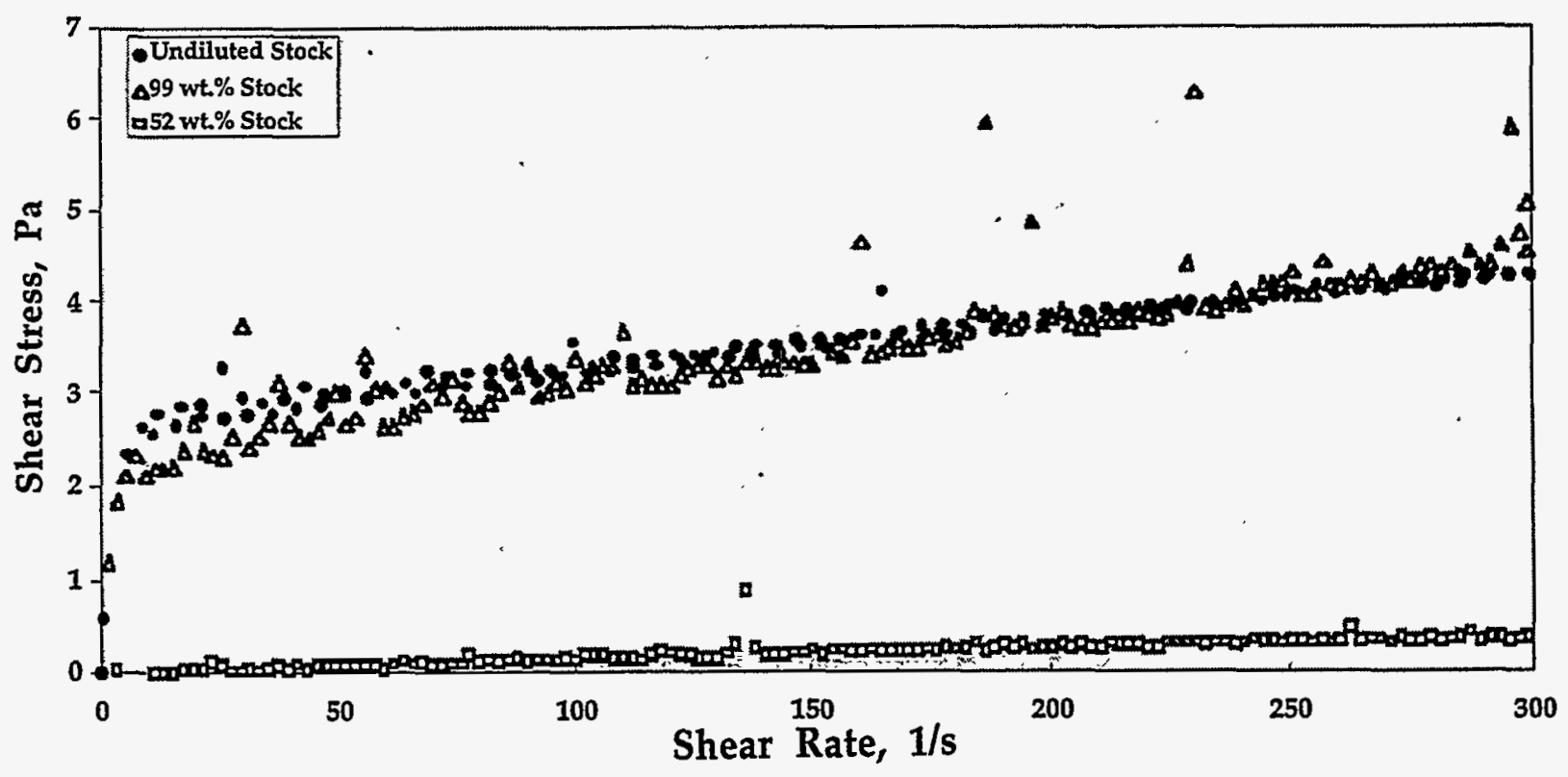

Figure 2.4. Rheograms of Diluted and Undiluted Tank SY-102 Wạte

(a) This is one of the many models that can be used to describe the material behavior. Other constitutive equations such as Power-Law and Herschel-Bulkley models may also be used. Later in this section, a Power-Law model of viscosity is presented. 
where, the value of $2.7 \mathrm{~Pa}$ is the apparent yield stress of the material. The actual yield stress can not be determined from this curve since it requires a particular instrument in which the stress is controlled, or from careful oscillatory rheometry tests. These capabilities are currently not available for radionuclide sample characterizations. Note that Equation 2.1 represents the high shear rate asymptotic behavior. At shear rates below $10 \mathrm{~s}^{-1}$ the material behaves more viscously, and its apparent viscosity at this limit is approximately $360 \mathrm{cP}$, or nearly two orders-of-magnitude higher than the high shear rate limit viscosity. The fact that the amount of hysteresis observed, that is the difference between ramp up and ramp down viscosities, was small suggests that the slurry has a stable network. Such materials commonly exhibit a significant viscoelasticity (Russel et al. 1989).

The viscoelastic response of a colloidal suspension can be described in terms of an elastic component and a viscous component. The elastic term, $G^{\prime}$ can be related to the volume fraction of the particles, $\Phi$, in the system (Tadros 1990):

$$
\mathrm{G}^{\prime} \sim \mathrm{k} \Phi \mathrm{n}
$$

where $\mathrm{k}$ is a constant and $\mathrm{n}$ depends on the degree of networking between the particles. To utilize this relationship, the contribution of the double layer to the effective volume fraction, $\Phi_{\text {eff }}$, is necessary:

$$
\Phi_{\mathrm{eff}}=\Phi[1+(1 / \mathrm{k}) / \mathrm{R}]^{3}
$$

where $\mathrm{k}$ describes the inverse thickness of the double layer and $\mathrm{R}$ is the radius. The zeta potential measurements of the SY-102 show that the particles are capable of becoming charged in the absence of salt. However, the high electrolyte concentrations in typical nuclear waste suspensions cause the $1 / \mathrm{k}$ term to tend toward zero and the pursuit of a zeta potential measurement becomes meaningless in these conditions. In this case, the effective volume fraction is not nearly as important as the physicochemical state of the particles. Sonntag and Russel (1987) observed that the aging of polystyrene lattices increased due to strengthening between particle contacts. In our nuclear waste suspensions, the high salt concentration screens the repulsive forces and aggregation occurs. Since the particles in our nuclear waste suspension have aged for as long as 40 years, networking and bridging between the particles would be expected. Therefore, in the measurement of the elastic modulus as a function of volume loading, we would expect an exponent near 4.5 (which corresponds to a highly networked system) (Macosko 1994). Dynamic rheometry would be able to determine what the actual value for the exponent is for SY-102 waste. Unfortunately, the dynamic oscillation tests could not be performed reliably with the ranges of deformation and frequencies available with the current instrumentation.

Dilution of the system produces interesting results. When the sample is only slightly diluted ( $99 \mathrm{wt} \%$ stock), the consistency of the material increases to $0.007 \mathrm{~Pa}-\mathrm{s}$, while the apparent yield stress decreases to approximately $2.3 \mathrm{~Pa}$. The low shear rate viscosity appears to be almost the same as that of the undiluted sample. When the sample is diluted by $48 \%$ ( $52 \mathrm{wt} \%$ stock), some difficulty was observed in the ramp up part of the measurement due to slip-stick phenomenon. However, at the end of the ramp up process the measurement appeared to be much smoother with little to no fluctuation in the signal. The data points shown on Figure 2.4 (and Figure 2.5) are data taken during the ramp down process. Note that the slurry approaches a Newtonian behavior, with a viscosity only slightly higher than water $(-1.4 \mathrm{mPa}-\mathrm{s})$ at $300 \mathrm{~s}^{-1}$. Figure 2.5 shows the effective viscosity of the mixtures shown in Figure 2.4. At shear rates of higher than $\sim 5 \mathrm{~s}^{-1}$, the undiluted slurry viscosity can be represented as $1500 * \dot{\gamma}^{-0.81}$. That is, a power law behavior index of 0.19 seems to fit the data closely. However, as can be noted, the viscosity of the fluid at low shear rates is over-predicted using this . model. Both the undiluted and $99 \mathrm{wt}$.\% stock seem to have a shear-independent viscosity at low shear rates, which is commonly observed in slurries of solid particles. 


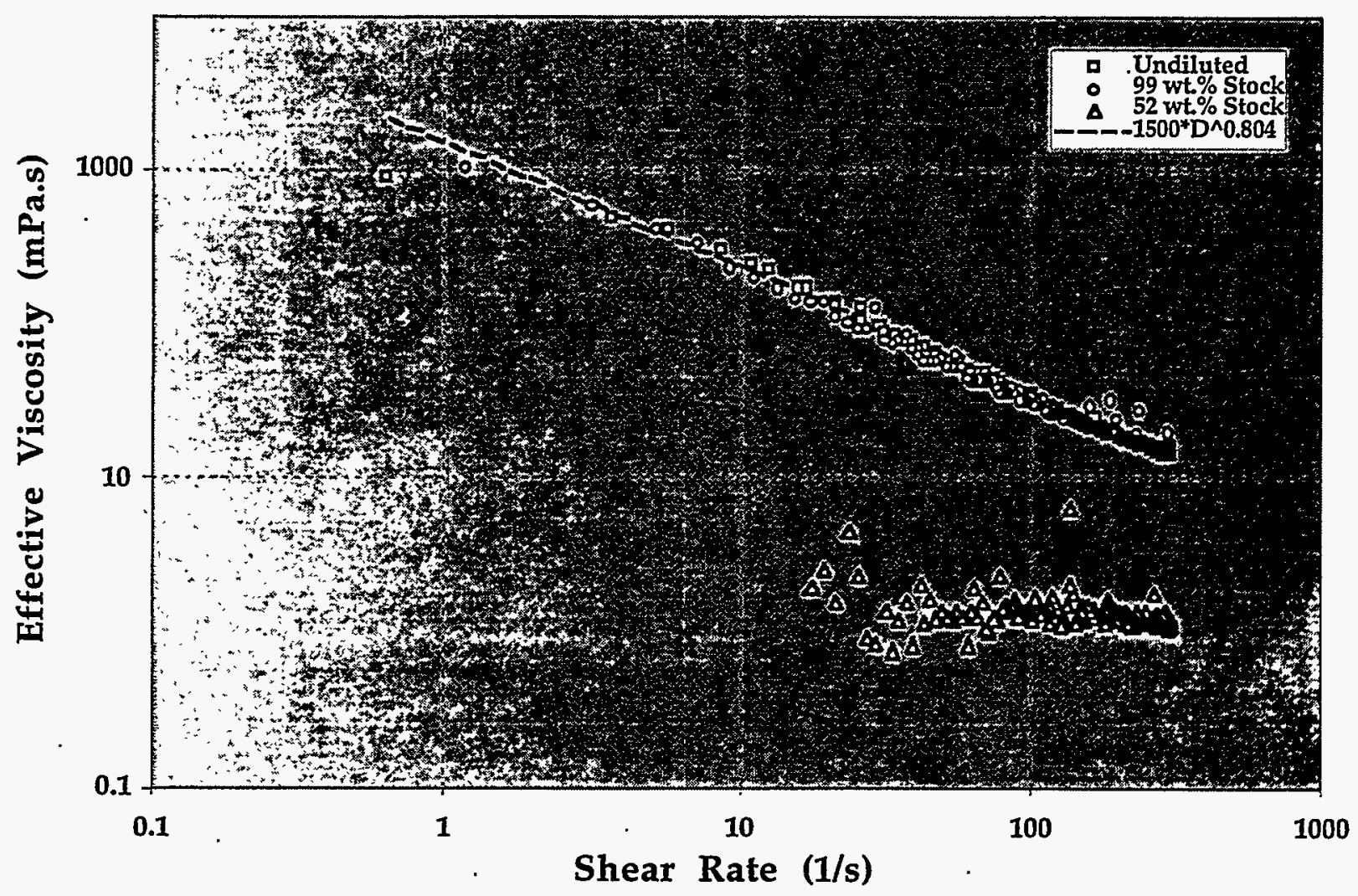

Figure 2.5. Viscosity of Diluted and Undiluted Tank SY-102 Waste

The data presented in Figure 2.5 are recast in terms of relative viscosity, $\mathrm{h}_{\mathrm{r}}$, as a function of the normalized solids volume fraction in Figure 2.6. Thus the maximum packing fraction selected in this figure is the packing fraction which correspond to the settled solids (undiluted slurry). As such, the agglomerates are treated the same way as solid particles in a noncolloidal suspension. The solid line in this figure is the empirical correlation proposed by Chong et al. (1971) based on extensive . investigation of rheological behavior of multimodal suspensions of glass spheres (Equation 2.4).

$$
\eta_{\mathrm{r}}=\left[1+0.75\left(\frac{\phi / \phi_{\mathrm{m}}}{1-\phi / \phi_{\mathrm{m}}}\right)\right]^{2}
$$

Here $\phi_{m}$ is the maximum packing fraction which, besides the physical geometrical arrangement (staggered or aligned lattice structure), depends on the size distribution and modality of the particles. Two parameters that have been cited in the literature are size ratio, $\lambda$ (the ratio of the largest to smallest particle diameters), and volume fraction of the smaller particles, $\xi$ (Chang and Powell 1993). It has been shown that $\phi_{\mathrm{m}}$ is approximately 0.74 for a monomodal suspension of spherical particles, although the experimental values are usually below 0.7 . As $\lambda$ or $\xi$ increase, the maximum packing fraction, $\phi_{m}$, increases above the monodisperse maximum packing fraction. Experiments have shown that the relative viscosity at high volume fractions is directly linked to the maximum packing fraction associated with a particular size ratio, $\lambda$, and small sphere volume fraction, $\xi$ (Shapiro and Probstein 


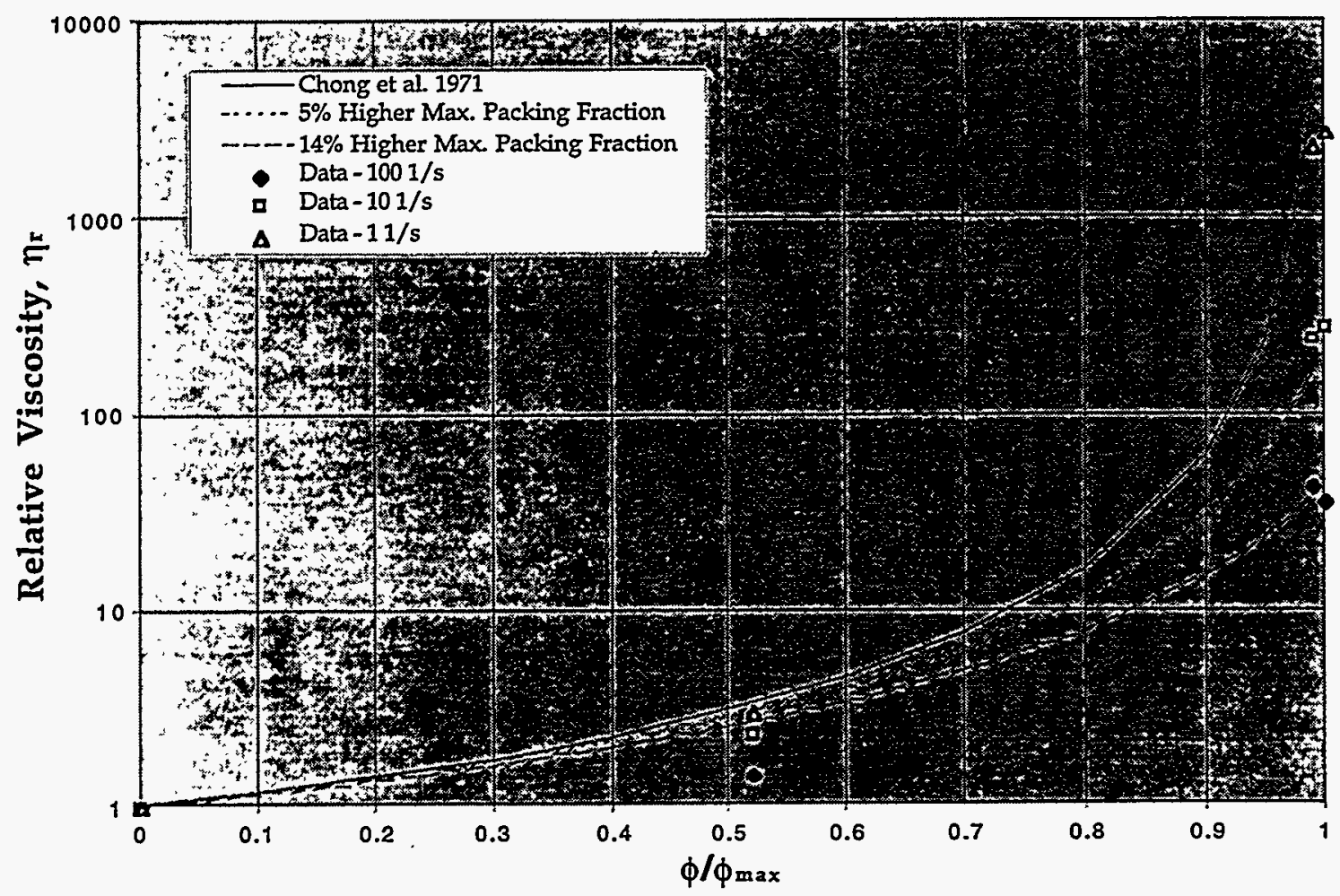

Figure 2.6. Effective Viscosity of Tank SY-102 Waste as a Function of Solids Concentration. The lines are empirical correlations for bimodal non-interacting suspensions for three different maximum packing fractions.

1992). Both experimental data and computational results show that the maximum viscosity reduction occurs at $\xi \sim 0.25$ to 0.35 (Shapiro and Probstein 1992; Chang and Powell 1993). Chang and Powell showed that this is the range within which the mean cluster size (or agglomerate size) is the minimum.

In the preliminary assessment (Onishi and Hudson 1996), the viscosity of $0.4-1.2 \mathrm{cP}$ for the supernate was used to estimate a critical velocity for solid deposition in the slurry pipeline for temperatures of ranging from 25 to $100^{\circ} \mathrm{C}$. The viscosity of the sludge/supernate mixture was assumed to be $4.8-14 \mathrm{cP}$ to determine an expected pressure drop in the slurry pipeline. As shown in Figure 2.6, the viscosities of the supernate and the sludge/supernate mixture having $\phi / \phi_{\mathrm{m}}=0.33$ is approximately 1 and $2 \mathrm{cP}$, respectively. Thus the preliminary assessment for the pipeline transport was conservative. Thus, the current study supports the preliminary assessment conclusion that is the sludge/supernate mixture will be successfully transferred through RCSTS.

The current rheological results obtained here are in contrast to the results reported in the Tank Characterization Report by DiCenso et al. (1995). They measured the shear strength of a sample of sludge from Tank SY-102 using a Haake Viscometer equipped with a shear vane. They reported the shear strength to be 39,000 dynes $/ \mathrm{cm}^{2}$, which is equivalent to $3,900 \mathrm{~Pa}$. Our measurements indicated a yield stress of approximately $2.7 \mathrm{~Pa}$. Smith et al. (1996) have shown that the values of stress obtained from a shear vane viscometer are not directly linked to the yield stress or shear strength of the material. It was shown that the error in measurement of yield stress dramatically increases with rotation rate of the shear vane due to inertial effects. Thus, accuracy of yield stress (shear strength) measurement reported by DiCenso is questionable. See Chapter 3 for further comparison between DiCenso's and our results. 


\subsubsection{Sedimentation}

The ratio of height to initial height of the sludge column versus acceleration is plotted in Figure 2.7. The data were used to calculate the compressive yield values. Realize that the acceleration is an average value; in the test tube, the acceleration changes as a function of position. A computational model has been developed that describes the density due to compression of the sediment layer at a given vertical position resulting from the weight of the sediment above it. This model can be used to predict the equilibrium sediment density profile and sediment height for a particular suspension given the total initial solids loading.

When the particle volume fraction is sufficiently high, a network of connected aggregates forms and the suspension takes on the form of a solid structure. In particular, compressive stresses on the system can be transmitted via the network throughout the system, and the structure has the ability to support itself. When such a network has formed, pressure can be applied either mechanically, as with a piston, or through gravitational forces. As the pressure is increased, the network structure will resist further compression until the forces become so strong that the structure will begin to deform irreversibly. The rheological property to describe this is the compressive yield stress, $P_{y}(\phi)$; which is defined as the value of the network pressure at which the flocculated suspension at volume fraction $\phi$ will no longer resist compression elastically and will start to yield and so irreversibly consolidate. A simple definition of the compressive yield stress at any vertical location is the relative weight of the sediment above that location. It is calculated by multiplying the integral of the volume fraction by the difference between the solid and liquid densities.

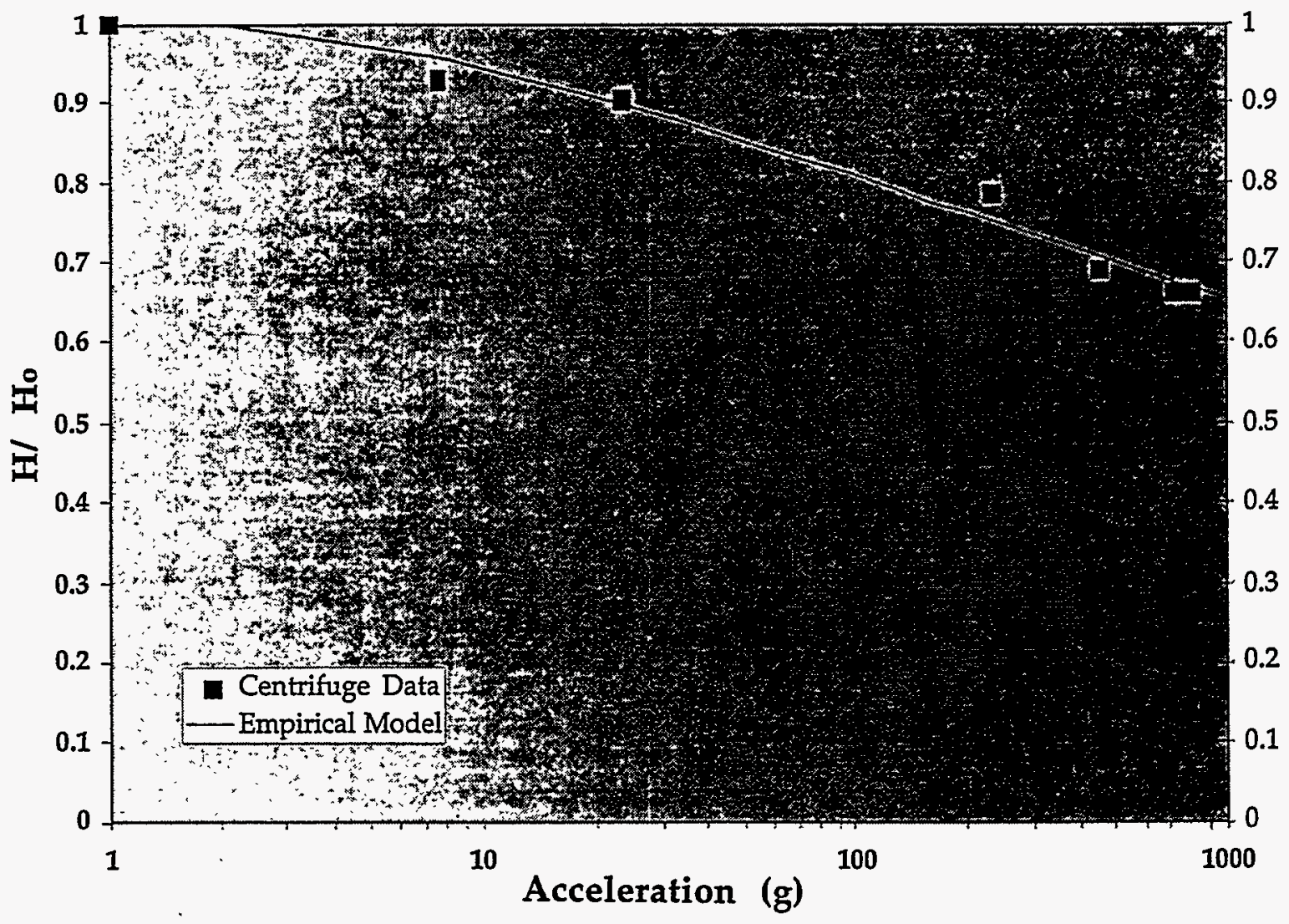

Figure 2.7. Compaction Results for Tank SY-102 Waste 
The compressive yield stress, $\mathrm{P}_{\mathrm{y}}(\phi)$, is an implicit function of many variables, including the size, shape, composition and relative number of particles involved, and the interparticle forces (which in turn depends on the solution chemistry). This form can be divided into two regions, with the boundary defined by the gel point volume fraction, $\phi_{\mathrm{g}}$. Below this value, the aggregates are not connected and act as independent units. At the gel point, these aggregates become interconnected throughout - the container to the extent that they are able to support a load. Above the gel point, the compressive yield stress is typically modeled using power law curves of the following type (Landman, White, and Buscall 1988):

$$
P_{y}(\phi)=c\left[\left(\frac{\phi}{\phi_{g}}\right)-1\right]^{m} \quad \phi>\phi_{g}
$$

or

$$
P_{y}(\phi)=c\left[\left(\frac{\phi}{\phi_{g}}\right)^{n}-1\right] \quad \phi>\phi_{g}
$$

With $\mathrm{m}$ varying between 4 and 10 and $\mathrm{n}$ varying between 8 and 10 .

The parameters for the power law curves may be determined using equilibrium sediment height data. The only information required is the solid and liquid densities, the weight or volume percent of particulate solids, and the final sediment height. The primary disadvantage of relying only on standard sediment height data is that the range is limited by the heights of the test columns used, which are typically much smaller than the full-scale applications that we wish to model.

The range of sediment compression data can be extended by measuring the sediment heights of samples which have been centrifuged at different speeds. The compressive yield stress is defined as the integral of the relative weight of the solids multiplied by the artificial gravity created by the centrifuge at each location in the sediment. This data, together with the equilibrium sediment height data, is used to determine the expression parameters. A computer program has been written to optimize the power law parameters $\left(c, \phi_{\mathrm{g}}\right.$, and $\mathrm{n}$ or $\mathrm{m}$ ) by performing a least-squares fit based on the. sediment heights using a simulated annealing approach. The exponents are restricted to the ranges specified above.

DiCenso et al. (1995) reports an interstitial liquid density of $1.18 \mathrm{~g} / \mathrm{mL}$ and a solids mass fraction of 0.605 for the settled sludge. The average solids density for the original sample based on measured water content is approximately $2.8 \mathrm{~g} / \mathrm{mL}$. The volume fraction of the settled solids may be determined from the following relationship.

$$
\phi=\frac{1}{1+\left(\frac{1}{c_{m}}-1\right) \frac{\rho_{s}}{\rho_{1}}}
$$

where $C_{m}$ is the mass fraction and $s$ and 1 correspond to solid and liquid, respectively. When the value of $2.8 \mathrm{~g} / \mathrm{mL}$ is used for the solid density, a volume fraction of $39 \%$ is found. 
An expression predicting the compression yield stress for SY-102 sediment was developed based on the procedure described above, with the exception that since the initial solids volume fraction was estimated to be 0.39 , the volume fraction at any point in the sediment is restricted to be at least 0.39 . The coefficients were determined by performing a least squares fit on the centrifuge data taken from samples provided to the Radiocolloids Laboratory. The compressive yield stress in $\mathrm{gm} / \mathrm{cm}^{2}$ is given by the expression

$$
\mathrm{P}_{\mathrm{y}}(\phi)=162.0\left[\left(\frac{\phi}{0.27}\right)-1\right]^{5.3}, \phi>0.27
$$

The compression yield stress curve as a function of solids volume fraction is shown in Figure 2.8.(a) As the weight of sediment increases, either through the addition of solid material or increasing the gravitational force, the solids volume fraction also increases. If the solids were resuspended, the resulting sediment density profile is presented in Figure 2.9. This expression was validated by predicting the sediment heights for each centrifuge run. The results are presented against the compaction results from the centrifuge in Figure 2.7. Maximum difference between predicted and measured heights is less than $5 \%$ in all cases. The compressive strength of the sludge at the bottom of the tank is estimated to be approximately $80.6 \mathrm{gm} / \mathrm{cm}^{2}(7.9 \mathrm{kPa}$ or $1.2 \mathrm{psi})$. This parameter is considered relevant due to the fact that many of the stresses on the sludge which cause the sludge to yield are in the normal compressive mode rather than shear. Note that this value is significantly higher than the shear strength of the waste, which implies that, depending on what failure theory is used, the compressive strength might be the dominant resistive strength of the material.

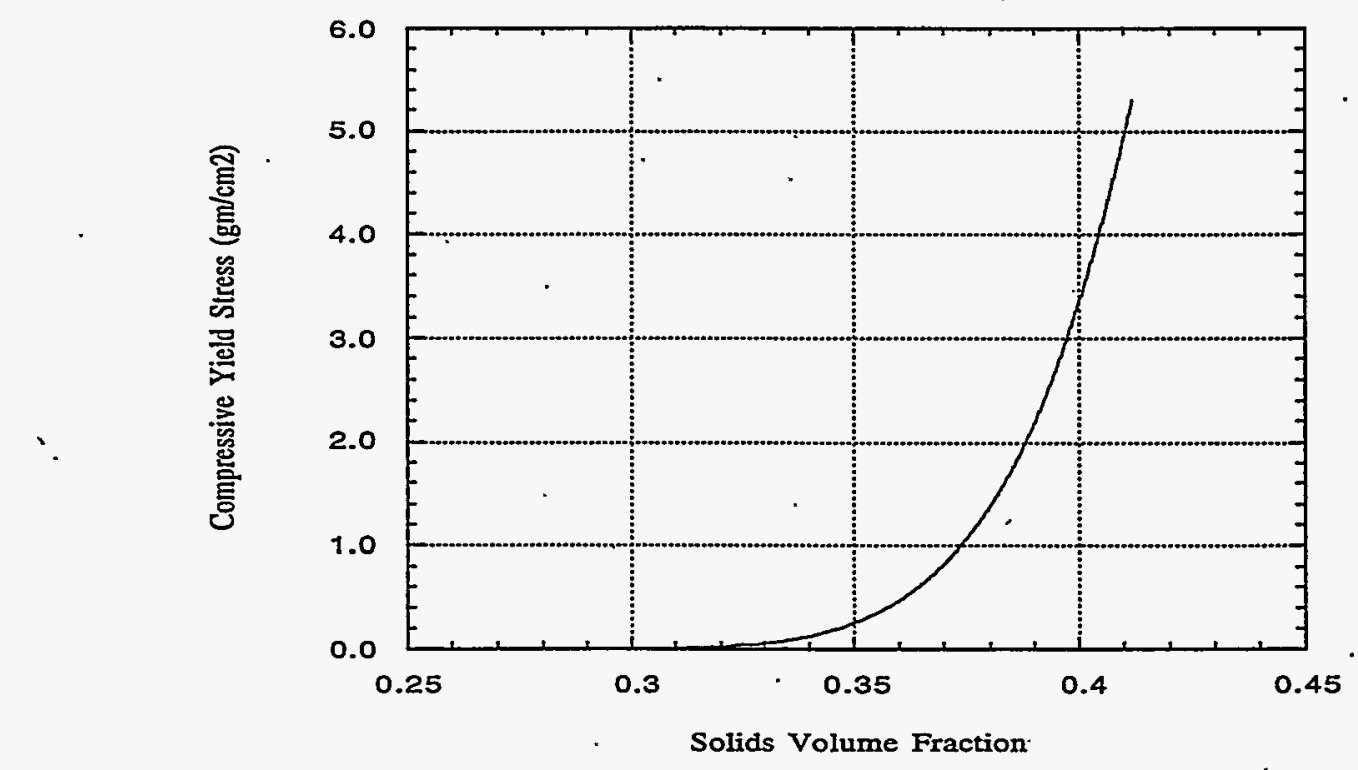

Figure 2.8. Compressive Yield Stress as a Function of Solids Volume Fraction for SY-102 Sediment

(a) To convert from $\mathrm{gm} / \mathrm{cm}^{2}$ to $\mathrm{Pa}$, multiply by 98.1 . 


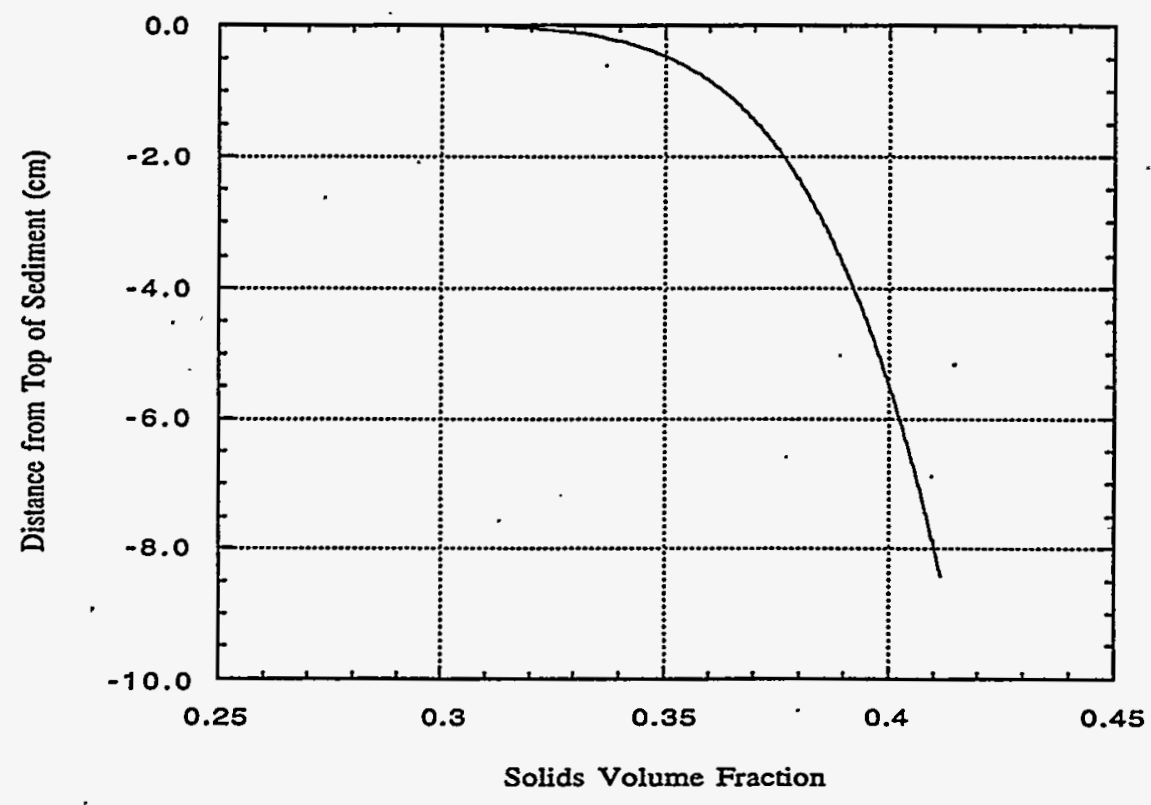

Figure 2.9. Solids Density Profiles versus Distance From the Top of the Sediment

To evaluate the assumption made about the maximum packing fraction, we further examine the available data from DiCenso et al. (1995) and the data provided in Figure 2.7. Referring to the figure, we find the maximum compaction ratio to be approximately 1.5 (ratio of maximum height to minimum height of the settled slurry). This compaction ratio can be used to calculate the average maximum packing volume fraction, 0.59 for the centrifuged waste. However, the sediment density also varies as a function of position within the sediment layer. The computed solids density profile for the maximum compaction case indicates a maximum volume fraction of 0.67 at the bottom of the sediment layer, which is within $10 \%$ of 0.74 , the maximum theoretical packing fraction for a suspension of uniform spheres. Further, we can estimate the volume fraction of the waste at the bottom of the tank by substituting the value of $7.9 \mathrm{kPa}$ for Equation (2.6) and solving for $\phi$. The volume fraction of the sludge at a depth of 42 in. below the sludge-supernatant layer interface is estimated to be $51 \%$. The value of $53 \%$ was used in the following chapter for modeling and analysis.

\subsection{Discussion}

The observation of rheopexy, thixotropy, and shear thinning behaviors may be perplexing. However, the knowledge provided by the particle size analysis, TEM results, zeta potential measurement, and the literature allows some intelligible statements to be made. Clearly, there is an appreciable quantity of silica in this system of colloidal particles. From the rheology, it is clear that the microstructure changes as a function of the applied shear. From the data obtained with shear rates of 10 and $100 \mathrm{~s}^{-1}$, we observe that the SY-102 sample contains agglomerates that are broken up due to the action of shear. This is understandable since the system contains a mixture of particles which would aggregate due to heterocoagulation. More importantly, the system has a high electrolyte content which allows the particles to be attracted to one another. Thus, the behavior of shear thinning (or alternatively Bingham plasticity) is understood in terms of aggregate disruption. The viscosity of the 
fluid was reduced at higher shear rates due to a broadening of the size distribution, where the population of smaller particles increased. Thixotropy was observed due to the time-dependent nature of the system.

The observation of rheopexy is quite different. Essentially, at low shear rates, the collisions between particles lead to aggregation. Simply, the silica renders the particles sticky. Similar behavior has been observed in sol gel films (Brinker and Scherer 1990) and simulated nuclear waste suspensions containing frit (a silica source) particles (Chang and Smith 1996). In addition, mineral phases such as montmorillonite and kaolin (Heckroodt and Ryan 1978) have shown this behavior. At higher shear rates, the attachments between particles are broken, and the system behaves in a shear thinning manner. 


\subsection{Pump Jet Mixing Simulations}

\subsection{SY-102 Tank Wastes and Retrieval}

Potential tank waste movements during the jet mixer operation in double-shell tank, SY-102 were evaluated using the three-dimensional TEMPEST computer code (Trent and Eyler 1993; Onishi et al. 1995). The main objective of the simulation is to determine if two pumps having four rotating $18.3-\mathrm{m} / \mathrm{s}(60-\mathrm{ft} / \mathrm{s})$ jets located at $43 \mathrm{~cm}$ (17 in.) above the tank bottom can sufficiently mix the sludge with the supernatant liquid for waste retrieval. The purpose of this simulation effort was also to determine the validity of the preliminary assessment's assumption of the tank waste being completely mixed in SY-102 Tank, and if necessary, was to further evaluate the potential chemical equilibrium and kinetic reactions and associated rheology changes by incorporating spatial and temporal distributions of the tank wastes.

SY-102 is one of 28 Hanford double-shell tanks. Its diameter and usable depth are approxi-

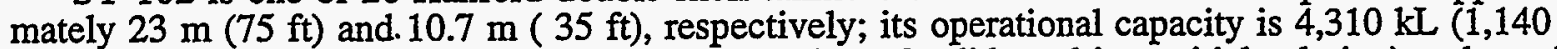
$\mathrm{kgal}$ ). The tank contains both sludge (a combination of solids and interstitial solution) and overlying supernatant liquid. Since it is an active double-shell tank in the 200 West Area, its waste volume can change due to waste introduction to and withdrawal from the tank, and from potential chemical reactions (e.g., solid precipitation/resolution and adsorption/desorption) occurring in the tank (Onishi and Hudson 1996). It contained $466 \mathrm{~kL}$ (123 kgal) of sludge in February 1990 and 2,560 kL (676 kgal) of supernate in March 1994; thus its volume ratio of sludge to supernate was 5.5 (DiCenso et al. 1995). Currently (as of September 1996), this ratio is approximately 1:2.

DiCenso et al. ( 1995) reported that Tank SY-102 has a sludge layer with a bulk density of 1.56 $\mathrm{g} / \mathrm{mL}$, while the overlying supernate has a density of $1.03 \mathrm{~g} / \mathrm{mL}$. The particle density of the average bulk sludge is $1.80 \mathrm{~g} / \mathrm{mL}$ (DiCenso et al 1995), and the sludge contains various solids, e.g., $\mathrm{NaNO}_{3}$ (s), $\mathrm{Al}(\mathrm{OH})_{3}$ (s), and iron solids (Onishi and Hudson 1996), as discussed in Section 2.

The particle distribution reported by DiCenso et al. (1995) and calculated unhindered settling (fall) velocity are shown in Table 3.1. The solid particles vary in size from 10 to $175 \mu \mathrm{m}$, with the average size about $53 \mu \mathrm{m}$ based on the volume (DiCenso et al. 1995). However, our particle size

Table 3.1. Particle Size Distributions of Tank SY-102 Sludge

\begin{tabular}{||c|c|c|c||}
\hline Size Fractions & $\begin{array}{c}\text { Particle Sizes } \\
(\mu \mathbf{m})\end{array}$ & $\begin{array}{c}\text { Percent } \\
\text { Volume }\end{array}$ & $\begin{array}{c}\text { Unhindered } \\
\text { Fall Velocity } \\
(\mathbf{m m} / \mathbf{s})\end{array}$ \\
\hline 1 & $10-20$ & 7 & 0.081 \\
\hline 2 & $20-30$ & 8 & 0.24 \\
\hline 3 & $30-40$ & 8 & 0.49 \\
\hline 4 & $40-50$ & 19 & 0.81 \\
\hline 5 & $50-60$ & 30 & 1.2 \\
\hline 6 & $60-100$ & 25 & 2.5 \\
\hline 7 & $100-175$ & 3 & 7.1 \\
\hline Total & & 100 & \\
\hline
\end{tabular}


measurements of the SY-102 sludge show a much finer and somewhat wider particle distribution, with most particles below $20 \mu \mathrm{m}$, as reported in Section 2. The unhindered settling velocities were calculated by assigning fluid viscosity to be $1.0 \mathrm{cP}$ (Onishi and Hudson 1996). As discussed in Section 2, it is recognized that these particle size distributions actually represent heterogeneous agglomerates and differ from distributions of pure discrete particles, thus affecting densities and associated rheology. The unhindered settling velocities were calculated by assigning fluid viscosity to be $1.0 \mathrm{cP}$ (Onishi and Hudson 1996). Since the measured particle distributions reported in Section 2 were obtained near the end of the modeling study period, we selected those values cited in DiCenso et al. (1995) for the SY-102 tank modeling, producing less favorable conditions for jets to mobilize the sludge without accounting for potential cohesiveness (if any). Thus, if the particle size distribution measured in this study more accurately represents the actual size distribution, one can use our model results for the finer solids e.g., Solid $1(10-20 \mu \mathrm{m})$ and Solid $2(20-30 \mu \mathrm{m})$ to represent the overall sludge behavior.

Tank SY-102 will use two mixer pumps each with two jet nozzles. The four outlets have 6"diameter nozzles and are placed 17 inches above the tank bottom, while the pump suction line has a $15^{\prime \prime}$ diameter and is positioned 7 inches above the tank bottom. The jet nozzles inject recirculating slurry into the tank with a velocity of $60 \mathrm{ft} / \mathrm{s}$. These two mixer pumps are located $6.1 \mathrm{~m}$ (20 feet) from the tank center on opposite ends of a diagonal line through the tank center. Each jet rotates over a half circle at the speed of $0.5 \mathrm{rpm}$.

\subsection{Tank SY-102 Model Setup with the Tempest Code}

The general TEMPEST computer code can simulate flow and mass/heat transport and chemical reactions (equilibrium and kinetic reactions) coupled together (Onishi et al. 1996). The T.2.10 version of TEMPEST used for this study solves three-dimensional, time-dependent equations of flow, momentum, heat, and mass transport, based on conservation of

- fluid mass (the equation of continuity)

- momentum (the Navier-Stokes equations)

- turbulent kinetic energy and its dissipation

- thermal energy

- mass of dissolved constituents

- mass of solid constituents

- mass of gaseous constituents.

Complete equations for conservation of mass, momentum, energy, and treatment of turbulence energy are documented in Trent and Eyler (1993). TEMPEST uses integral forms of the fundamental conservation laws applied in the finite volume formulation. It uses the $k-\varepsilon$ turbulence model (Rodi 1984) to solve the turbulence kinetic energy and its dissipation. TEMPEST can accommodate non-Newtonian power law fluids, as well as fluids whose rheology depends upon solid concentrations (Mahoney and Trent 1995; Onishi et al. 1995). Transport of multiple liquid, gas, and solid constituent species can be performed.

A Tank SY-102 conceptual model used in TEMPEST simulates the operation of two mixer pumps each with two rotating jet nozzles, as stated above. TEMPEST simulated the movements of supernate and seven different solid size fractions (See Table 3.1). Tank SY-102 TEMPEST runs were full three-dimensional but covered one-half of the tank domain through symmetry of the pump jet 
operation. By using the latest known volume ratio (1:2) of the sludge to the supernate, the thicknesses of the sludge and supernate were assigned to be $1.17 \mathrm{~m}$ (46") (DiCenso et al. 1995) and 2.34 m (92"), respectively, in the model.

Main parameters for the SY-102 modeling are particle sizes and densitiès and solid volume fraction in the sludge, and those used to calculate solid settling velocities, viscosity and yield stress. Particle size distributions are represented using the seven discrete particle sizes shown in Table 3.1 reported by DiCenso et al. (1995). As discussed in Section 2, the extent to which the particles within these size distributions may actually be flocs rather than primary particles is difficult to quantify.

The bottom portion of the sludge is believed to be heavier than the rest of the sludge, and the solid volume fraction in the sludge is approximately 0.48-0.54 (DiCenso et al. 1995). Thus, we divided the sludge layers to two groups: 8" bottom layers (from the tank bottom to $20.3 \mathrm{~cm}$ above) have the solid volume fraction of 0.53 , while 38 " upper sludge layers $(20.3 \mathrm{~cm}$ to $1.17 \mathrm{~m}$ above the tank bottom) have the volume fraction of 0.44 . Thus the weighted average of the sludge volume fraction was 0.46 .

Due to the lack of specific information on the density of different size particles, all TEMPEST calculations assumed that the particles have a uniform density. The particle density of $1.80 \mathrm{~g} / \mathrm{mL}$ was reported by DiCenso et al. (1995). However, the density of approximately $2.03 \mathrm{~g} / \mathrm{L}$ is needed to match the measured bulk sludge density of $1.56 \mathrm{~g} / \mathrm{mL}$ with the solid fraction value of 0.53 for the bottom sludge layer. Thus we selected $2.03 \mathrm{~g} / \mathrm{mL}$ for the solid density. Note that most of the pure solids (e.g., $\mathrm{NaNO}_{3}(\mathrm{~s}), \mathrm{Al}(\mathrm{OH})_{3}(\mathrm{~s})$ ) are heavier than $1.8 \mathrm{~g} / \mathrm{mL}$, but aggregates are expected to be lighter than the pure solids, as discussed in Section 2 . With this density of $2.03 \mathrm{~g} / \mathrm{mL}$, the bottom sludge layers have a sludge density of $1.56 \mathrm{~g} / \mathrm{mL}$ with a solid volume fraction of 0.53 , while the upper sludge layers have a density of $1.47 \mathrm{~g} / \mathrm{mL}$ with a volume fraction of 0.44 .

Information is not available on the shape of different materials, so all particulates were assumed to be spherical. The unhindered settling velocities occurring under small solid concentrations for the seven particle sizes were provided to TEMPEST by the Stokes Law (Vanoni 1973) with spherical particles. These input settling velocities do not-include the effects of particle interaction such as particle flocculation or agglomeration. The TEMPEST model does include the effect of hindered settling, which occurs under high solid concentrations. The input unhindered settling velocity of each particle was internally adjusted to account for effects of hindered settling based on an equation of the form:

$$
\mathrm{v}_{s}=\mathrm{V}_{s o}(1-\beta)^{\mathrm{a}}
$$

where

$$
\begin{aligned}
\mathrm{V}_{\mathrm{s}} & =\text { hindered settling velocity } \\
\mathrm{V}_{\mathrm{so}} & =\text { input settling velocity (unhindered settling velocity) } \\
\beta & =\text { Cv/Cvmax } \\
\mathrm{C}_{\mathrm{v}} & =\text { solid volume fraction in slurry } \\
\mathrm{C}_{\mathrm{vmax}} & =\text { maximum solid volume fraction }(=0.53 \text { in this study) } \\
\mathrm{a} & =6.0
\end{aligned}
$$

To some extent the results for different assumptions for particle size, density, and shape of a minor component can be evaluated by calculating the settling velocity and determining an equivalent diameter for that particle.

Turbulent flow Reynolds stresses are modeled through an effective viscosity. The PrandtlKolmogrov hypothesis is used to relate the effective viscosity to a velocity and a length scale. In this approach, transport equations for the turbulent kinetic energy $(\mathrm{k})$ and the dissipation of turbulent kinetic energy $(\varepsilon)$ are solved by the $\mathrm{k}-\varepsilon$ model (Rodi 1984) to determine the effective turbulent (eddy) viscosity, $\mu_{\mathrm{T}}$ as 


$$
\mu_{T}=\dot{C}_{\mu} \rho k^{2} / \varepsilon
$$

where $C_{\mu}=$ constant equal to 0.09

$$
\begin{aligned}
& \rho=\text { fluid density } \\
& \mathrm{k}=\text { turbulent kinetic energy } \\
& \varepsilon=\text { dissipation of turbulent kinetic energy }
\end{aligned}
$$

For the TEMPEST calculations of Tank SY-102, a measured value for the supernate density $(1.03 \mathrm{~g} / \mathrm{mL}$ from DiCenso et al. 1995) and the estimated molecular viscosity of the supernate of $1 \mathrm{cP}$ (see Onishi and Hudson 1996) were used for the supernatant liquid.

The slurry molecular viscosity is then calculated by multiplying the molecular viscosity of the base fluid by a factor of "a" raised to the power " $b$ ":

$$
\mu=\mu_{\mathrm{T}} \mathrm{b}^{\beta}
$$

where $\mu_{\mathrm{T}}=$ base fluid viscosity ( $=1.03 \mathrm{cP}$ in this study)

$\mathrm{b}=$ ratio of the sludge viscosity to supernate viscosity

$\beta=\mathrm{C}_{\mathrm{v}} / \mathrm{C}_{\mathrm{vmax}}$

This equation, which does not explicitly include the strain rate, was selected in a previous effort to model the periodic rollover and gas release processes in Tank SY-101. (Trent and Michener 1993). The value of " $\mathrm{b}$ ". is determined by dividing a high viscosity intended to represent a very high solids concentration $\left(C_{v}=C_{v \max }=0.53\right)$ by a viscosity of supernatant $\left(C_{v}=0\right)$. As discussed in Section 2, reliable data on which to base the value of $C_{v \max }$ is not available, but $C_{v \max }$ of 0.53 was selected in this study to be a reasonable value to model SY-102 (DiCenso et al. 1995). The previous SY-101 modeling effort with $\mathrm{C}_{\mathrm{vmax}}=0.48$ produced results similar to observed tank phenomenon of sludge rollovers and pump jet mixing.

As discussed in Section 2, viscosity of the sludge varies significantly with strain rate, ranging from the viscosity of approximately $4,500 \mathrm{~Pa}-\mathrm{s}$ at $0.06 \mathrm{~s}^{-1}$ to $0.04 \mathrm{~Pa}-\mathrm{s}$ at $100 \mathrm{~s}^{-1}$ (see Figures 2.2 and 2.3). Measured shear stress as a function of the strain rate is shown in Figure 2.5. Preliminary SY-102 TEMPEST modeling predicted that the strain rate near the tip of the injected jet around the tank wall is on the order of $1-10 \mathrm{~s}^{-1}$. With these strain rates, the measured sludge viscosity is on the order of $10 \mathrm{~Pa}$-s. Furthermore, many values of viscosities of diluted sludge measured in this study at about $10 \mathrm{~s}^{-1}$ strain rate and those values (as consistency) extracted from DiCenso et al. (1995) are approximately $10-100 \mathrm{cP}$ for SY-102 tank waste by mixing SY-102 sludge with approximately twice the volume of its supernate (see Table 3.2).

Thus we selected the viscosity of the sludge $\left(C_{v}=C_{v m a x}=0.53\right)$ to be 12 Pas-s, as a base case. We also selected the sludge viscosity to be 4,500 Pas-s in some cases to include a very high viscosity condition. The variation of viscosity with the solid fraction for these two cases is shown in Figures 3.1 and 3.2 with some measured values of DiCenso et al. (1995). The molecular viscosity of the slurry is added to the turbulent viscosity and the sum is used in fluid dynamic calculations.

Table 3.2. Power Law Curve Fit Parameters for Tank SY-102 Wastes (DiCenso et al. 1995)

\begin{tabular}{|c|c|c||}
\hline $\begin{array}{c}\text { SY-102 } \\
\text { Waste }\end{array}$ & $\begin{array}{c}\text { Consistency } \\
\text { Pa-s }\end{array}$ & $\begin{array}{c}\text { Flow Behavior } \\
\text { Index }\end{array}$ \\
\hline Sample 1 & 0.013 & 0.808 \\
\hline Sample 2 & 0.014 & 0.791 \\
\hline
\end{tabular}




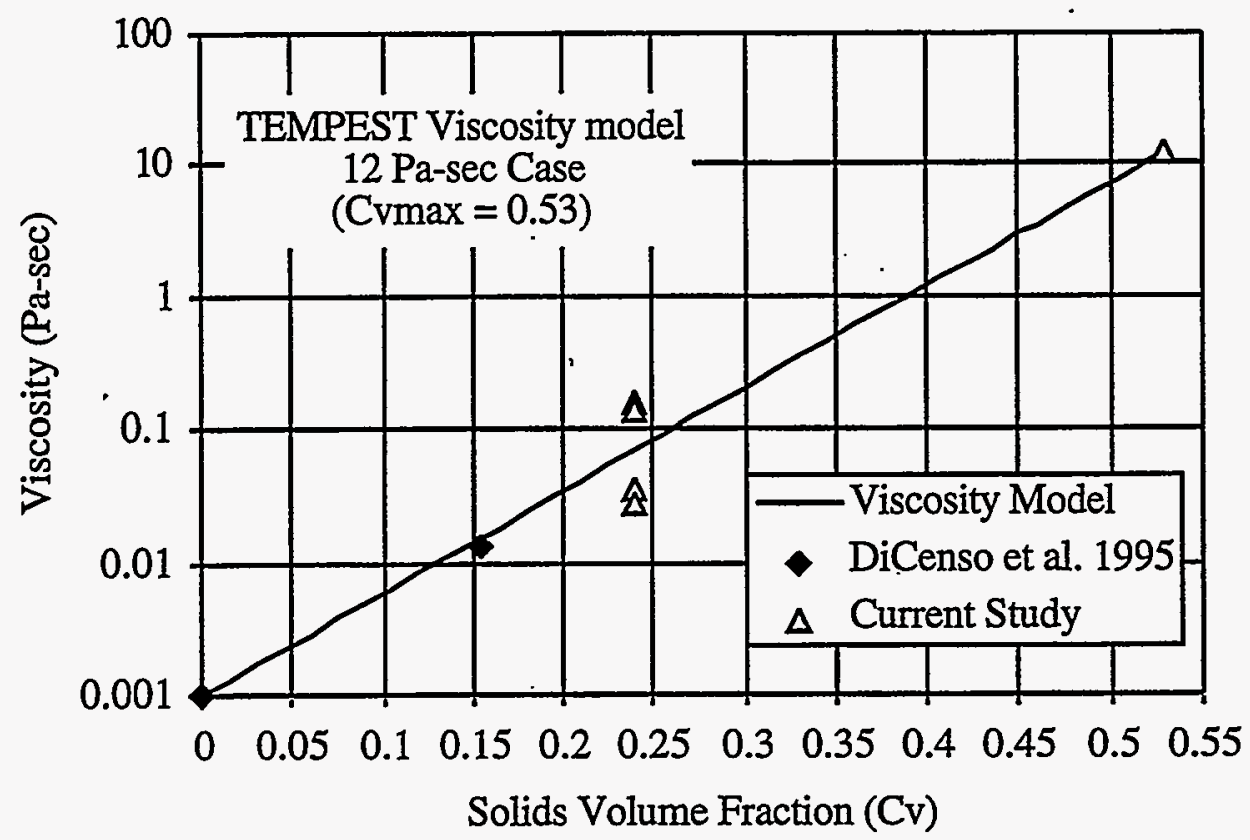

Figure 3.1. Variation of Viscosity with Solid Concentrations for the Sludge Viscosity of $12 \mathrm{~Pa}-\mathrm{s}$ and $\mathrm{C}_{\mathrm{vmax}}$ of 0.53 , With Some Measured Viscosity

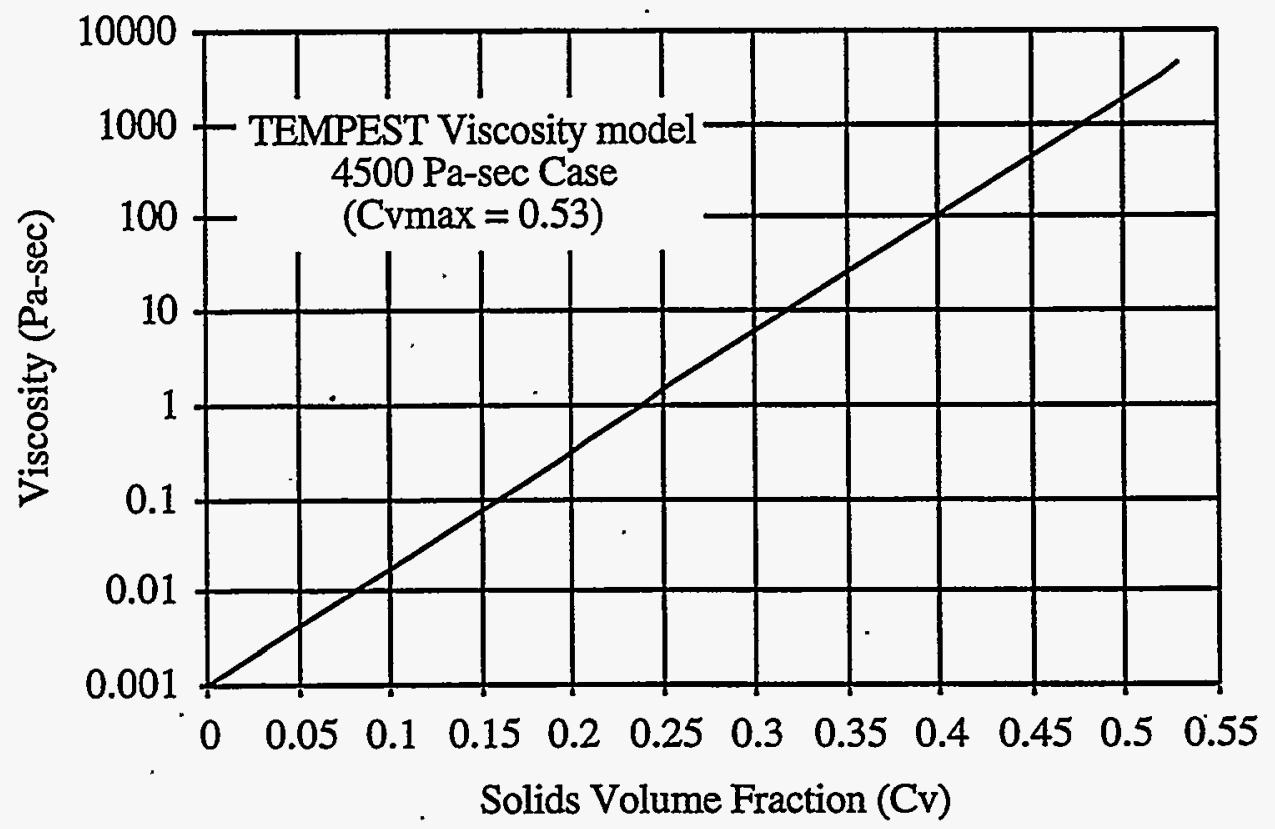

Figure 3.2. Variation of Viscosity with Solid Concentrations for the Sludge Viscosity of 4,500 Pa-s and $\mathrm{C}_{\mathrm{vmax}}$ of 0.53 
Currently TEMPEST has the following yield stress formulation, but it has had only limited success in the past:

$$
\tau_{\text {yield }}=b_{1} \beta^{b_{3}}\left\{\frac{1-e^{-b_{2} \gamma}}{b_{2} \gamma+s}\right\}(1-\alpha)
$$

where $\tau_{\text {yield }}=$ calculated yield stress

$$
\begin{aligned}
b_{1} & =\text { yield stress }(=0 \text { or } 1.5 \text { psi in this study) } \\
b_{2} & =\text { dimensionless strain coefficient }(=50 \text { in this study) } \\
b_{3} & =\text { dimensionless moisture exponent }(=25 \text { in this study) } \\
s & =\text { a small constant set at } 1 \times 10-30 \\
\alpha & =\text { volume fraction of gas }(=0 \text { in this study) } \\
\gamma & =\text { strain rate of fluid }
\end{aligned}
$$

The calculated yield stress is added to the stress imparted by the viscosity in response to fluid shear. Since the sludge in Tank SY-102 is believed to have very small yield stress (about $2.7 \mathrm{~Pa}$ indicted in Section 2), we have not included the yield stress in most of the simulation cases. Comparisons of the simulation results with and without yield stress for this tank and other tanks were reported in Whyatt et al. (1996), indicating that the effect of yield stress expressed in Eq. 3.4 on particle segregation was not large.

While TEMPEST has the capability to model diffusive effects on solutes and particles, diffusion process was eliminated in the modeling to compensate potential numerical diffusion effects on the tank waste transport.

Several SY-102 modeling cases with different tank conditions (e.g., sludge-supernate volume fraction), model parameter values (e.g., $C_{\text {vmax }}$, settling velocity, viscosity, and the yield stress) and grid setups (grid resolution, simulated tank domain, and jet rotation) are reported in Serne et al. (1996) and Whyatt et al. (1996).

\subsection{Model Applications and Result Evaluations}

We have tested many cases, and we will discuss the main TEMPEST simulation cases for Tank SY-102 modeling. These ten cases are

- Case 1: Base case having two rotating $60-\mathrm{ft} / \mathrm{s}$ jets located $20 \mathrm{ft}$ off-center and the sludge with its viscosity of $12 \mathrm{~Pa}-\mathrm{s}$ initially settled on the tank bottom

- Case 2: Case with the sludge and supernate initially fully mixed, otherwise the same as Case 1

- Case 3: Highly viscous sludge (4,500 Pa-s) case, otherwise the same as Case 1

- Case 4: Highly viscous sludge (4,500 $\mathrm{Pa}-\mathrm{s})$ case with the sludge and supernate initially fully mixed, otherwise the same as Case 1

- Case 5: A case with a rotating pump located at the tank center and the sludge with its viscosity of 12 Pa-s initially settled on the tank bottom

- Case 6: A case with a rotating pump located at the tank center and the sludge with its viscosity of $12 \mathrm{~Pa}-\mathrm{s}$ and supernate initially fully mixed

- Case 7: $45-\mathrm{ft} / \mathrm{s}$ (75\% of the designed the jet velocity used for Case 1) jet velocity case, otherwise the same as Case 1 
- Case $8: 30-\mathrm{ft} / \mathrm{s}$ (50\% of the designed the jet velocity used for Case 1 ) jet velocity case, otherwise the same as Case 1

- Case 9: $30-\mathrm{ft} / \mathrm{s}$ (50\% of the designed the jet velocity used for Case 1) jet velocity case, with the sludge and supernate initially fully mixed, otherwise the same as Case 1

- Case 10: $15 \mathrm{ft} / \mathrm{s}$ (25\% of the designed the jet velocity used for Case 1 ) jet velocity case, otherwise the same as Case 1

These ten cases have $C_{v \max }$ of 0.53 and do not include the yield stress. TEMPEST was run to cover two simulation hours for the ten cases with time steps ranging from several milliseconds to 20 milliseconds, taking computational time of a few days to a week for each case. As will be discussed, the simulations reached quasi-steady state conditions in less than two simulation hours.

\subsubsection{Grid Resolutions}

We simulated movements, settling and mixing of seven solids and supernate in Tank SY-102, with TEMPEST to evaluate if two pumps having four rotating $18.3-\mathrm{m} / \mathrm{s}(60-\mathrm{ft} / \mathrm{s})$ jets can sufficiently $\mathrm{mix}$ the sludge with supernate for the waste retrieval. To select grid resolution suitable to represent the complex sludge and supernate movements and still computationally fast enough to be practical, we tested various grid resolutions. The grid resolution selected for this study divides sludge and supernatant regions into 11,760 computational cells and was used for all simulation cases except Cases 5 and 6 . The finest grid resolution case we tested has 47,040 cells. The computational time to cover the same simulation time for the finer grid case is about one order of the magnitude greater than that needed for the coarser grid resolution. Comparisons of various grid resolutions indicated that overall flow movements and sludge setting patterns are relatively similar, but the fine grid resolution can define the jet spread pattern more sharply, especially near the tank bottom, and thus can potentially generate more accurate flow and sludge movements.

These effects of grid resolutions on waste movements can be seen by comparing predicted results of Case 1 (the base case) and the finer grid resolution case at the same 15-minute simulation time. At that time, the rotating jets are directed at 3 and 9 o'clock positions. Figures C.1 through C.3 in Appendix $\mathrm{C}$ show the predicted velocity and volume-fraction distributions of Solid 1 (the finest solid, with a diameter of 10-20 $\mu \mathrm{m}$ ), Solid 5 (the medium size solid of diameter 50-60 $\mu \mathrm{m}$ ) and Solid 7 (the coarsest solid; diameter of $100-175 \mu \mathrm{m}$ ), respectively in the vertical plane at the 3 o'clock position for the base (coarser) case. All plots of simulation results are presented in Appendix C, except color plots and selected few figures which are included in Section 3. Corresponding predicted results for the finer grid are shown in Figures C.4 through C.6. All these figures show that one of two rotating jets injected at $43 \mathrm{~cm}$ (17') above the tank bottom is hitting the tank wall and is bent upward along the tank wall, carrying the sludge on the tank bottom with it. They also show that the finest solid (Solid 1) is more uniformly mixed than the coarsest solid (Solid 7) in both the finer and coarser grid resolutions.

Predicted horizontal distributions of Solid 7 volume fraction are shown in Figs. C.7 through C.9 on the tank bottom, $43 \mathrm{~cm}$ (17") above (height of the jet injection nozzles) the tank bottom, and on the waste surface ( $3.5 \mathrm{~m}$ or $138^{\prime \prime}$ above the Tank Bottom), respectively for the coarser grid resolution. Corresponding results for the finer grid are shown in Figs. C.10 through C.12, also showing large scale circulations.

Comparisons of these figures show that the overall patterns of predicted velocity and solids distributions are very similar in these grid resolutions. Moreover, the predicted maximum variations of the volume fractions are very similar in the two grid resolution cases, as shown in Table 3.3.

The comparison also shows that the finer grid resolution produces more clearly defined and focused jets, especially on the tank bottom, generating faster maximum jet-induced velocity on the 
Table 3.3. Maximum Variations (\%) of Predicted Volume Fractions over the Entire Tank in Two Grid Resolution Cases

\begin{tabular}{|c|c|c|c|}
\hline & Solid 1 & Solid 5 & Solid 7 \\
\hline Coarse Grid & 0.60 & 8.6 & 39 \\
\hline Fine Grid & 0.76 & 9.9 & 43 \\
\hline
\end{tabular}

tank bottom. This higher bottom velocity in the finer resolution can potentially move the bottom sludge more easily than the slower bottom velocity predicted in the coarser grid.

Considering the computational time requirements for the SY-102 modeling, and since the overall solid accumulation and distribution patterns are similar for the both finer and coarser grids, we selected the coarser grid resolution for the study. We judged that the coarser grid resolution is adequate to obtain overall sludge mixing and accumulation patterns in Tank SY-102 and that it produced a somewhat conservative (smaller) estimate on the movement of the sludge located on the tank bottom.

\subsubsection{Simulation Results of Case 1: Base Case}

The Tank SY-102 simulation was intended to determine whether two mixer pumps with four rotating $18.3-\mathrm{m} / \mathrm{s}(60-\mathrm{ft} / \mathrm{s})$ jets can sufficiently $\mathrm{mix}$ the sludge with supernate for the waste retrieval, and to determine the validity of the complete mixing assumed under the preliminary assessment (Onishi and Hudson 1996). To achieve these objectives, TEMPEST was used to predict the potential tank waste movements (both the sludge and supernate) during the jet mixer operation in the doubleshell tank. As stated above, TEMPEST simulated jet-induced mixing and movements of the seven solids (see Table 3.1) and supernate. Two rotating jets with $0.5 \mathrm{rpm}$ were assigned at $20 \mathrm{ft}$ off the tank center.

Among the ten cases evaluated, Case 1 is considered to be the representative case of the SY-102 pump jet mixing and thus is our base case. This case has the coarser grid resolution, as discussed above, and has the viscosity variation with bulk solid concentrations shown in Figure 3.1. Case 1 has solid volume fractions of 0.53 in the bottom 8" sludge layers and 0.44 in the upper 38" thick sludge. The volume fractions of the seven solids are shown in Table 3.4.

As stated in Section 3.2, the 8" bottom and 38" upper sludge layers have the bulk densities of 1.56 and $1.47 \mathrm{~g} / \mathrm{mL}$, respectively, in the model as the initial condition. The supernate has the initial thickness of 92 " overlying the sludge layers, thus the thickness of the total tank wastes.(combination of the sludge and supernate) is $138^{\prime \prime}$ in the model.

Table 3.4. Assigned Initial Volume Fractions in the Sludge Layers

\begin{tabular}{||l|c|c|c|c|c|c|c|c||}
\hline \hline & Solid 1 & Solid 2 & Solid 3 & Solid 4 & Solid 5 & Solid 6 & Solid 7 & Total \\
\hline $\begin{array}{l}\text { Upper 38-inch } \\
\text { Sludge }\end{array}$ & 0.034 & 0.038 & 0.034 & 0.081 & 0.148 & 0.086 & 0.018 & 0.439 \\
\hline $\begin{array}{l}\text { Bottom 8-inch } \\
\text { Sludge }\end{array}$ & 0.041 & 0.050 & 0.041 & 0.104 & 0.171 & 0.099 & 0.023 & 0.529 \\
\hline
\end{tabular}


We ran the model for two simulation hours. As the rotating jet mixed the sludge and supernate, all the solid concentrations kept changing with time. The model predicted that there is hardly any settling of fine solids, but that there is some settling of the coarser solids, as expected.

Three-dimensional distributions of predicted volume fractions of Solid 7 (the coarsest solid) in the half of the tank after one second (basically showing the initial condition), three minutes, six minutes, ten minutes, and two simulation hours are shown in Figures 3.3 through 3.7 with volume fractions color coded (red for above 0.016 and blue for below 0.004). These figures show the boundary of the three dimensional simulation area (a half of the tank with the depth from the tank bottom to the supernate surface). The tank center at the bottom and the supernate surface are marked by small circles in left portions of the figures. Horizontal distributions of Solid 7 volume fraction and velocity shown in these figures are predicted values on the tank bottom. The predicted volume fraction are also shown in a vertical plane (orienting from 1:30 o'clock position to 7:30 o'clock position) containing the rotating pump.

Figure 3.3 shows the tank bottom is all red, since the initial volume fraction of Solid 7 on the tank bottom is 0.023 (see Table 3.4), which is greater than 0.016 . Furthermore, the distributions of Solid 7 volume fraction in the vertical planes show $1 / 3$ of the total depth is in red, representing 46 " of the initial sludge layer, while the top $2 / 3$ is blue, showing 92 " of the overlying supernate in the tank. As pump jet mixing progresses, the color changes on the tank bottom and the vertical plane in these figures. At each time plane presented in these figures, the two rotating jets are directed toward the 3 and 9 o'clock positions. At these times, the jets hit the tank wall and center, and spread to the rest of the tank.

TEMPEST, as shown in these figures, predicted the following overall behavior of the pump jet mixing of SY-102 under the conditions and parameters used for the modeling:

As the sludge-mixing time progresses,

- the jet containing the sludge slurry is rapidly mixing the sludge and supernate,

- the solid volume fraction on the tank bottom are reduced through mixing,

- vertical distributions become increasingly uniform,

- the finer the solid particle, more uniform its distribution becomes,

- the solids are mostly removed and suspended from the tank bottom by the direct hits by the jets,

- the suspended solids (especially the coarser solids) in the tank areas not directly hit by the jets start to settle, until they will be directly hit and resuspended again by the rotating jets,

- thus the solid settling areas keep changing, depending on the directions of the rotating jets at any given time over two minute rotating intervals, except possibly more stable setting areas in the furthest corners of the tank,

- mixing efficiency is related to the frequency of the jet rotation, i.e., the faster the rotation, the shorter the required time to achieve near full mixing, as long as the jets are hitting each tank spot with a reasonable time duration during the rotation, and

- much of the mixing is achieved in 10-20 minutes (20-40 sweeps by the rotating jets) within most areas of the tank, and the final, quasi-steady-state is reached in less than one to two hours.

The current SY-102 modeling revealed that the sludge and supernate will be well mixed in less than two hours with the bulk of the mixing achieved in 10-20 minutes. Especially the very fine solids (e.g., Solid 1 with 10-20 $\mu \mathrm{m}$ diameters and Solid 2 with 20-30 $\mu \mathrm{m}$ diameters) have very 


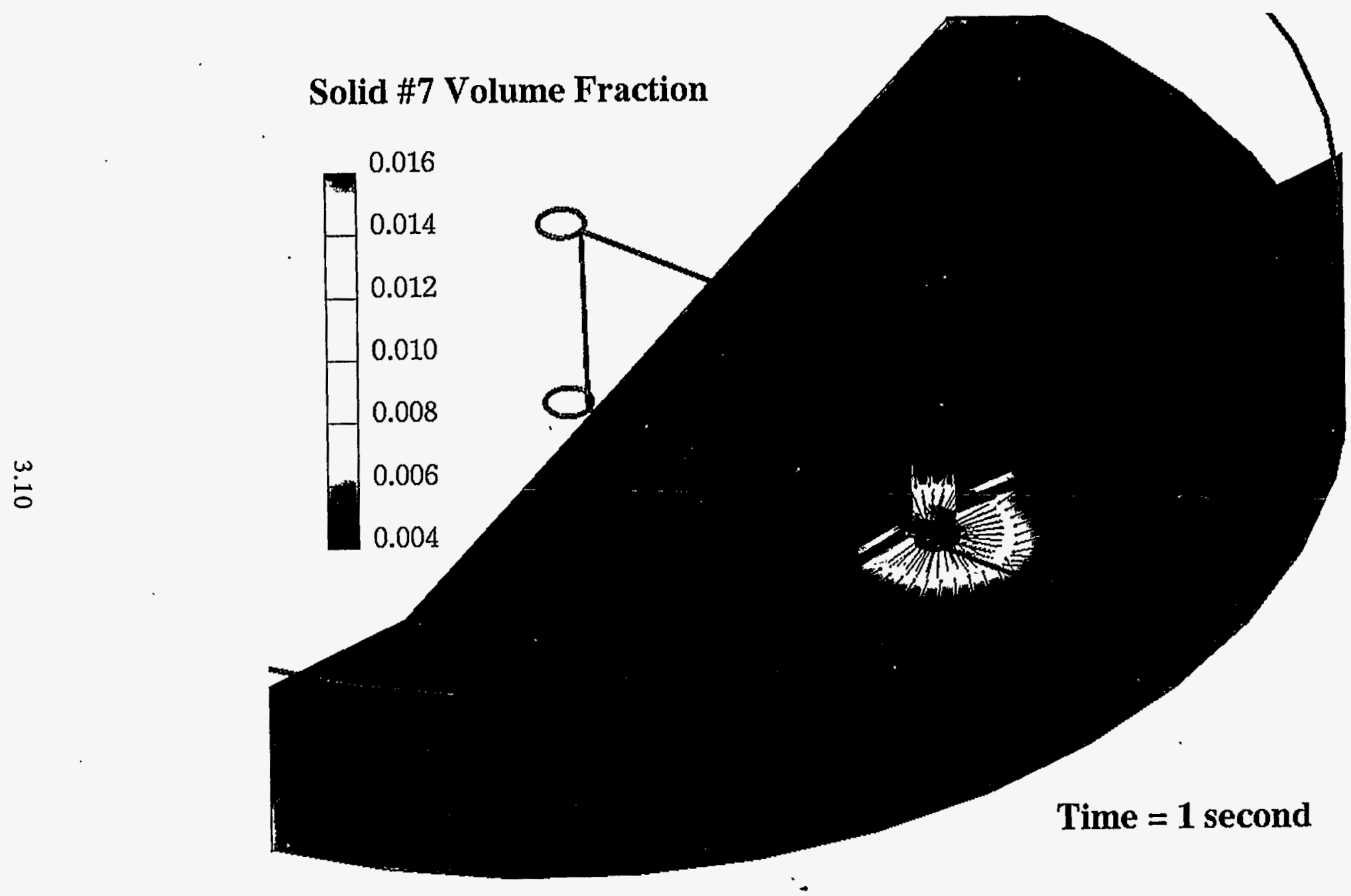

Figure 3.3. Initial Distributions of Velocity $(\mathrm{m} / \mathrm{s}$ ) on the Tank Bottom and Three Dimensional Distribution (on tank bottom and in two vertical planes) of Solid 7 Voliume Fraction for Case 1 


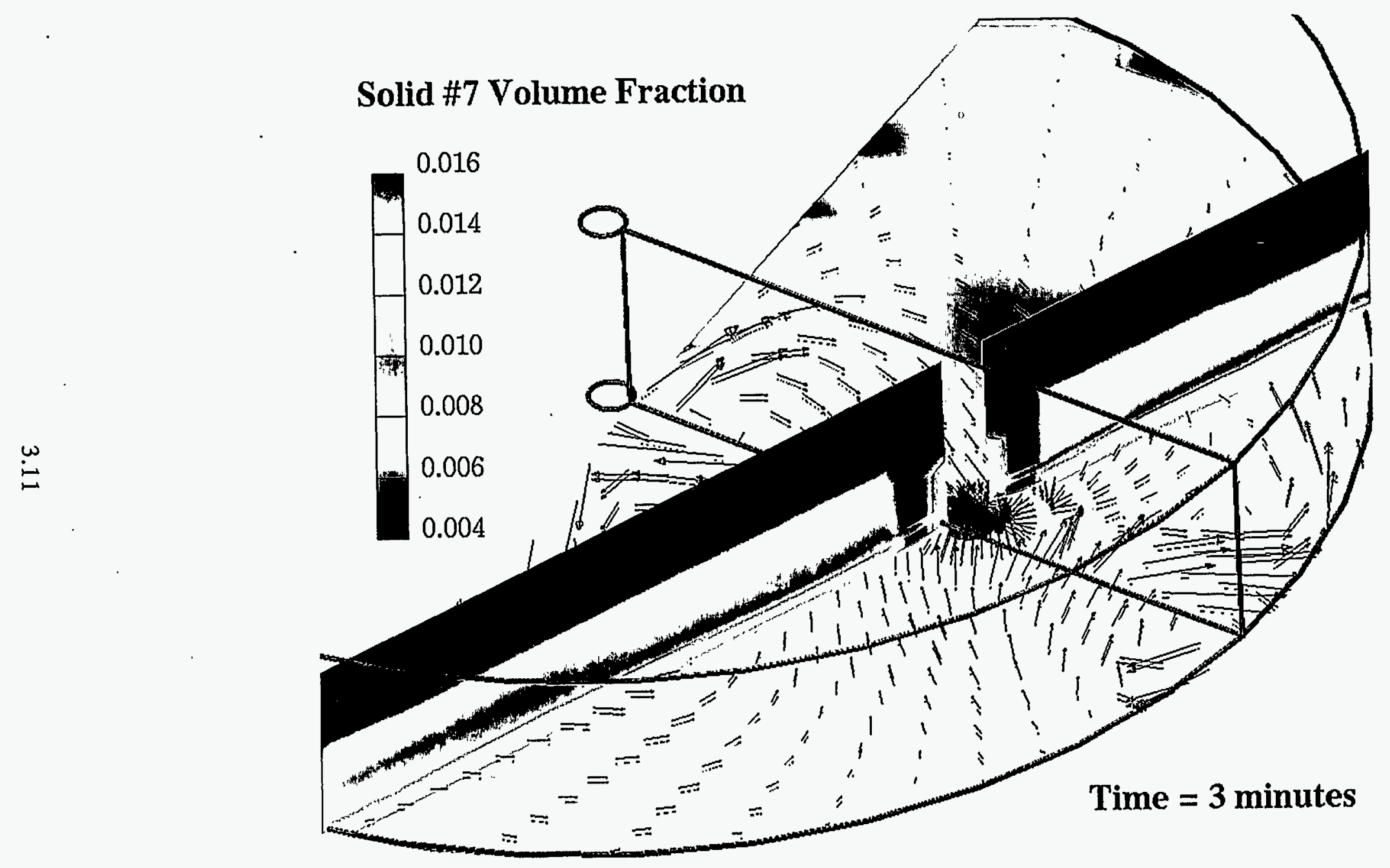

Figure 3.4. Predicted Distributions of Velocity $(\mathrm{m} / \mathrm{s})$ on the Tank Bottom and Three Dimensional Distribution (on tank bottom and in two vertical planes) of Solid 7 Volume Fraction at Three Simulation Minutes for Case 1 


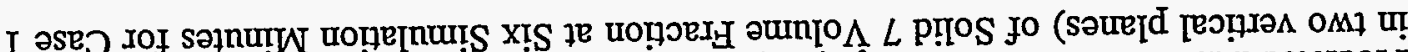

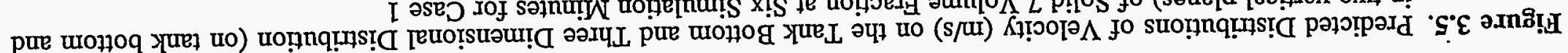

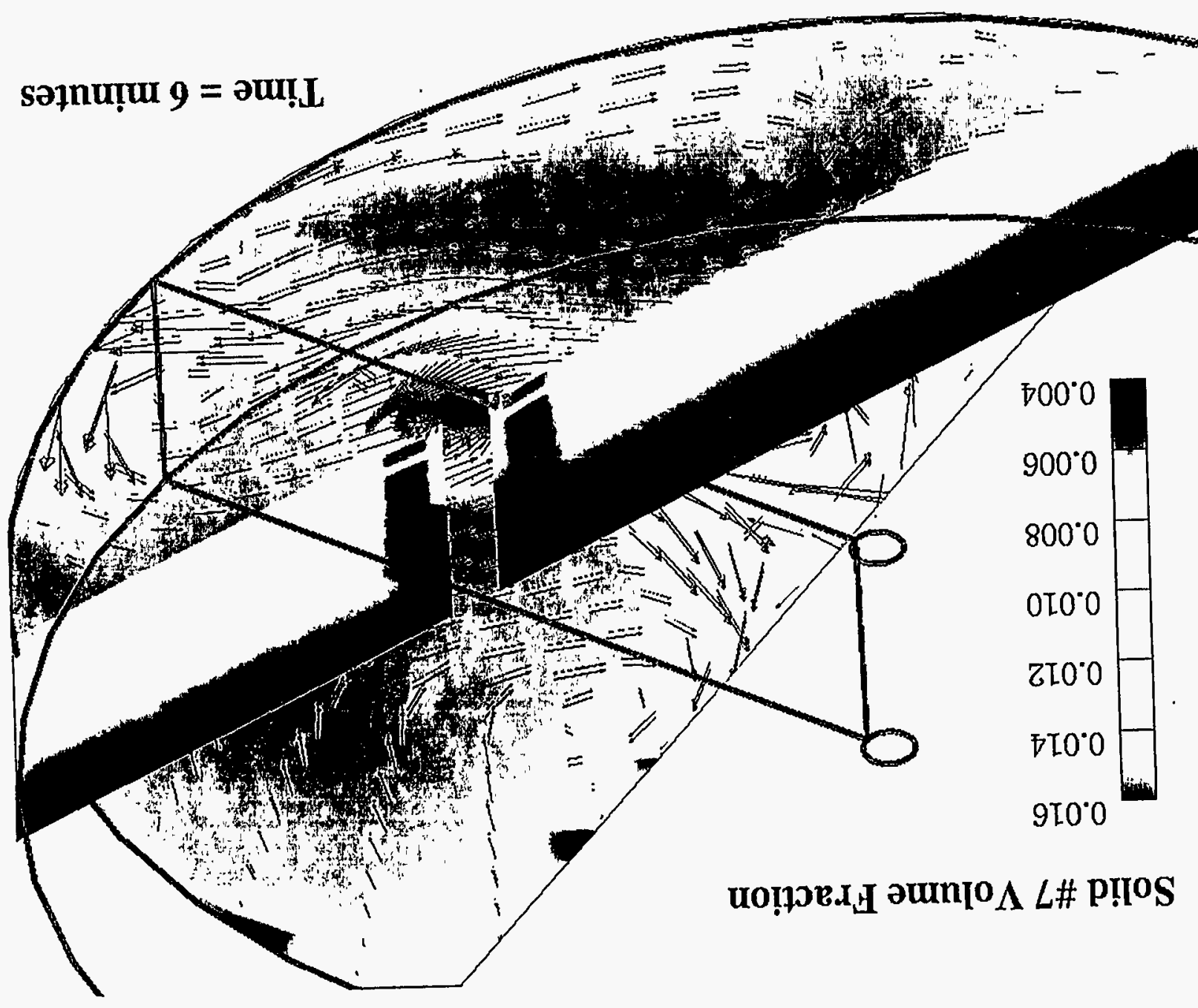




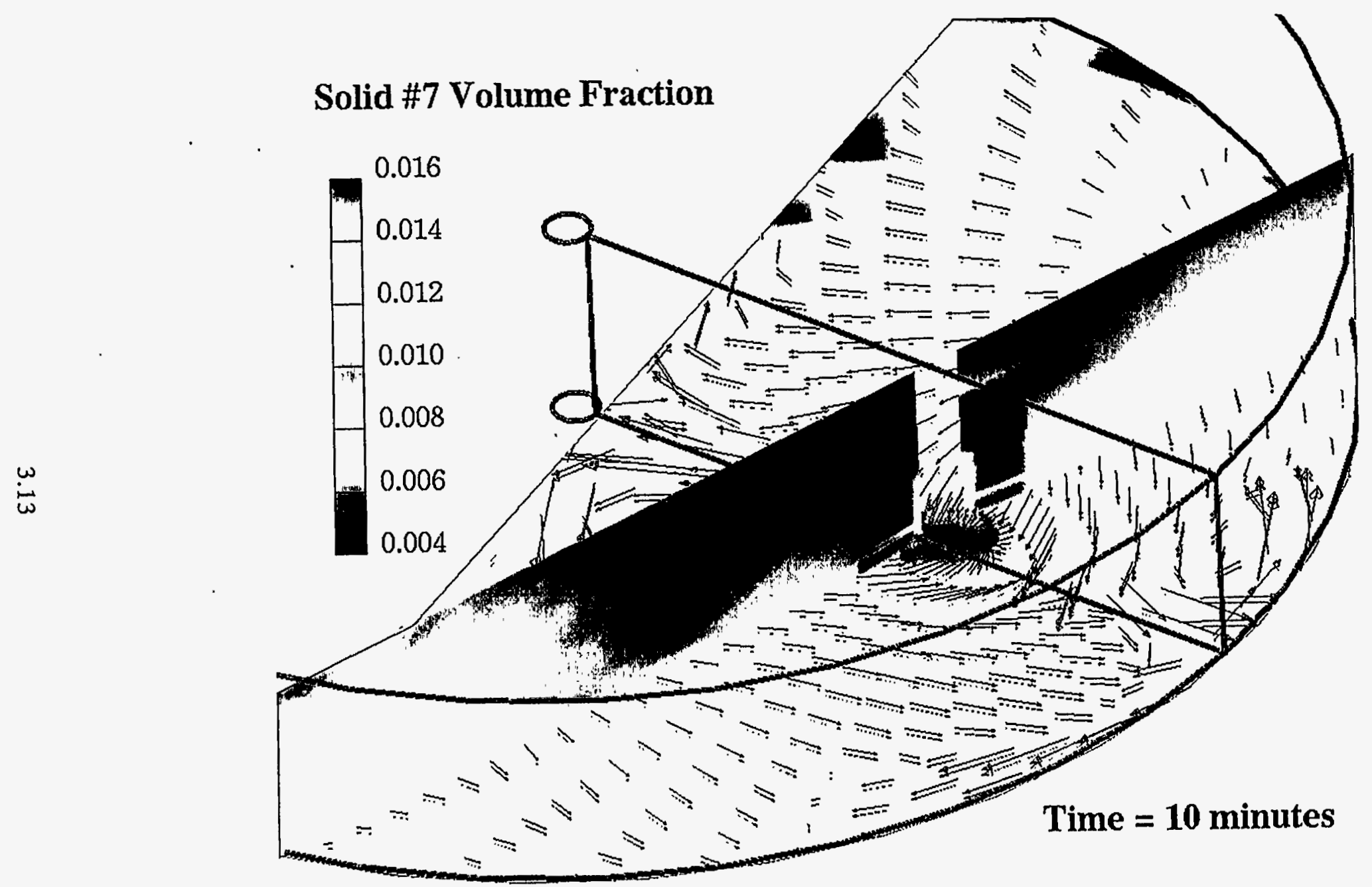

Figure 3.6. Predicted Distributions of Velocity $(\mathrm{m} / \mathrm{s})$ on the Tank Bottom and Three Dimensional Distribution (on the tank bottom and in two vertical planes) of Solid 7 Volume Fraction at Ten Simulation Minutes for Case 1 


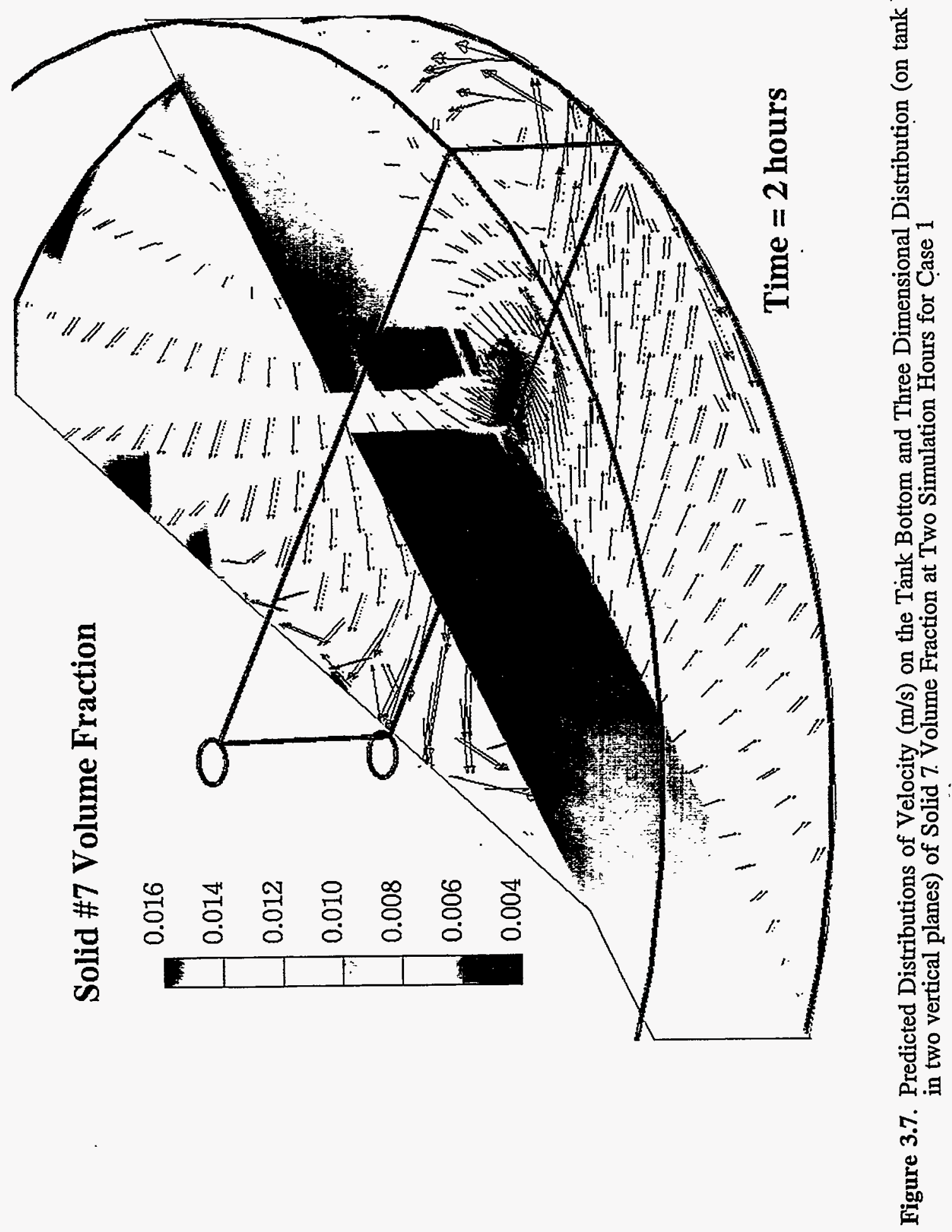


uniform distributions within the entire tank. Recall that the current modeling used the DiCenso et al. (1995)'s solid size distribution, which is much more coarser than our measurements indicate (see Section 2). This modeling results, thus validates the complete mixing assumption made for the chemical modeling of the preliminary assessment (Onishi and Hudson 1996). Moreover the preliminary assessment concludes that main solids to have dissolution/precipitation reactions were Nacontaining solids, especially $\mathrm{NaNO}_{3}(\mathrm{~s})$. Since $\mathrm{NaNO}_{3}(\mathrm{~s})$ is expected to be dissolved or precipitated between 15 minutes and one hour (Onishi and Hudson 1996), the current model prediction that the bulk of the mixing will be achieved in 10 minutes to two hours supports the use of the equilibrium chemical modeling performed under the preliminary assessment (see Onishi et al. (1996) for $\mathrm{NaNO}_{3}(\mathrm{~s})$ and $\mathrm{NaNO}_{2}(\mathrm{~s})$ dissolution/precipitation kinetic modeling). Aluminum-containing solids, e.g., $\mathrm{Al}_{2} \mathrm{Si}_{4} \mathrm{O}_{10}$ on the other hand may take a very long period time to complete the solid dissolution/ precipitation. Thus, their chemical reactions may not significantly alter the composition of the sludge and supernate within 10 minutes to two hours of the mixing. Note that preliminary assessment predicted that change on $\mathrm{Al}$ solid (gibbsite) amount caused by the waste mixing and heating was very small. Thus, the current time-dependent, three-dimensional tank modeling effort confirms the validity of the previous chemical modeling, and supports the conclusion of the preliminary assessment that the SY-102 waste mixing and heating do not adversely impact the waste retrieval operation.

The extent of the sludge mobilization area and time required to achieve the needed degree of the sludge mixing are controlled by many factors, not the least of which are yield stress, viscosity, and flocculation and break-up of solid aggregates and associated changes in aggregate density and settling velocity, as discussed in Section 2. Since TEMPEST has limited success of handling yield stress (especially for the sludge to resist initial movement induced by the flow), we assigned the yield stress for the current simulation to be zero, as discussed in Section 3.2. As discussed in Section 2, the SY-102 sludge is believed to have a very small yield stress (approximately $2.7 \mathrm{~Pa}$ ); thus this assumption of no yield stress was judged reasonable. Should there actually be a significant yield stress in the sludge, there may be more resistance to sludge mobilization than currently modeled. With the yield stress, it may also take more time to mobilize the sludge and may reduce the tank area of mobilized sludge. Even when the flow-induced force exerting on the solids is greater than the yield stress, it also takes some time for the sludge to move, as the sludge is more and more mixed with the supernate to reduce its viscosity to move it easier and faster (see Figure 3.1). We evaluated the extent of the sludge mobilization area by the rotating jets, based on not only the TEMPEST-predicted sludge distributions but also on predicted velocity distributions and general information on potential critical velocity for resuspension (Vanoni 1973) and yield stress for other tanks, as we discuss later.

We will now discuss the model results in detail.

At two simulation hours, the two rotating jets are directed at 3 and 9 o'clock positions. Predicted velocity and Solid 1 distributions at two simulation hours in the vertical Plane 21 (9 o'clock position) and Plane 15 (11 o'clock position) are shown in Figures C.13 and C.14, respectively. Distributions of Solids 5 and 7 at this time are shown in Figures C. 15 through C.18, which show that the $60 \mathrm{ft} / \mathrm{s}$ jet entrains the sludge and supernate from both above and below the jet and is spreading out toward the tank center at this time. As the jet spreads, it reaches the tank bottom and mobilizes the sludge on the bottom as well. Around the tank center, the jet directed at 9 o'clock collides with a jet located $20 \mathrm{ft}$ off-center on the opposite side of the tank directed at $30^{\prime}$ clock, thus the entrained mixture of the sludge and supernate moves upward to reach the waste surface 138" above the tank bottom. This near-surface flow moves toward the tank center and the 12 o'clock position, then moves downward to supplement the wastes entrained by the jet. This produces large-scale, slow-moving eddies/ circulations in the tank, enhancing the mixing of the wastes (see Figures C.13 and C.14).

Horizontal velocity and volume fraction distributions at the tank bottom, 17" above the tank bottom, and 138" (the waste surface) above the tank bottom shown in Figures C.19 through C.27 for Solids 1,5 , and 7 confirm these patterns of jet spreading, hitting the tank wall, and vertical and horizontal flow circulations. Predicted solid fraction distributions of Solids 1 through 7 are presented in Figures C.28 through C.27, respectively. 
Although ways that the eddies/circulations are produced vary from time to time depending on the rotating jet directions, the rotating jets continuously induce a large-scale flow circulations to achieve mixing. However, it should also be noted that the velocity outside of the jets is not very large (mostly much less than $1 \mathrm{~m} / \mathrm{s}$ ) so that larger solids can start to increase their concentrations near the tank bottom through settling, until the rotating jets hit these areas and mobilize the solids again. This intermittent mobilization by the jets and settling of larger particles is repeated throughout the jet mixing operation. Finer solids (e.g., Solid 1), however, maintain uniform distributions throughout the tank, as comparisons of Figures C.13 through C.34 clearly indicate.

As shown in Figures C.28 through C.34, these seven solids settles in four areas, two centering around the horizontal circulation produced by the jet reflection at the tank wall and tank center and the other two along the farthest tank wall area (11 and 7 o'clock positions) due to the difficulties of jets reaching the farthest corners of the tank. The former two areas are also the transient areas where the longest time has passed for the solids to settle, since the rotating jets hit there last time and keep appearing and disappearing over each two-minute jet rotation. On the other hand, the latter solid accumulation areas are more stable over time. Although the solid accumulation patterns are similar among various size solids, the amounts of solid accumulation on the tank bottom are significantly different. The finer the solid, the more uniform its distribution is, as expected. The degrees of solid volume fraction variations over the entire tank and on the tank bottom are shown in Table 3.5. Note that, the although Solid 7 accumulates on the tank bottom more readily, its. initial share of the total solids is quite small (only 3\%), as shown in Table 3.1. Predicted maximum solid volume fractions and segregation factors for all solids at two simulation hours over the initial sludge concentrations on the tank bottom are shown in Table 3.6.

As shown in this table, relative concentrations of finer solids (Solids 1 through 4) were slightly reduced (up to 12\%) from the initial condition, while coarser solids (Solids 6 and 7) increased their relative volume fractions among solids on the tank bottom, due to settling of the coarser solids. However, the relative increases of the coarser solid fractions are not significant (only 10 to $20 \%$ increases). Thus, even at the tank bottom, the solid composition does not change much from the initial condition.

Table 3.5. Maximum Variations (\%) of Predicted Volume Fractions over the Entire Tank and on the Tank Bottom for Case 1

\begin{tabular}{|l|c|c|c|c|c|c|c|}
\hline & Solid 1 & Solid 2 & Solid 3 & Solid 4 & Solid 5 & Solid 6 & Solid 7 \\
\hline $\begin{array}{l}\text { Variations over } \\
\text { Entire Tank }\end{array}$ & 0.51 & 1.6 & 3.4 & 5.6 & 8.1 & 16 & 37 \\
\hline $\begin{array}{l}\text { Variations within } \\
\text { Tank Bottom }\end{array}$ & 0.34 & 1.1 & 2.4 & 3.9 & 5.7 & 11 & 27 \\
\hline
\end{tabular}

Table 3.6. Maximum Volume Fractions and Segregation Factors on the Tank Bottom for Case 2

\begin{tabular}{||l|l|l|l|l|l|l|l|l||}
\hline & Solid 1 & Solid 2 & Solid 3 & Solid 4 & Solid 5 & Solid 6 & Solid 7 & $\begin{array}{c}\text { Total } \\
\text { Solids }\end{array}$ \\
\hline $\begin{array}{l}\text { Volume } \\
\text { Fraction at 2 hr }\end{array}$ & 0.012 & 0.013 & 0.012 & 0.029 & 0.054 & 0.033 & 0.0087 & 0.162 \\
\hline $\begin{array}{l}\text { Segregation } \\
\text { Factors }\end{array}$ & 0.93 & 0.88 & 0.95 & 0.92 & 1.0 & 1.1 & 1.2 & \\
\hline
\end{tabular}


If the particle size distribution (mostly finer than $20 \mu \mathrm{m}$ ) measured under this study more .accurately represents the actual sludge solid size distribution, then almost all solids will behave like Solid 1 , thus the sludge and supernate will be mixed uniformly throughout the tank without any measurable settling of the solids to the tank bottom.

Another way to examine the potential sludge mobilization by the jets are to examine the velocity distributions. If we assume that the dynamic pressure (velocity head) is a main force to overcome the yield stress to initiate the sludge movement and that the jet density hitting the non-moving sludge is approximately $1.3 \mathrm{~g} / \mathrm{mL}$, then the jet flows with $0.2,0.5,1.0,1.5$, and $2.0 \mathrm{~m} / \mathrm{s}$ velocities exert approximately $26,160,650,1,500$, and $2,600 \mathrm{~Pa}$ of the force on the sludge to move it. Thus, velocity contours of these values may correspond to the areas where the sludge of these corresponding yield stresses start to move. At 55.8 simulation minutes (or approximately one simulation hour), the jets are directed at $2: 30$ and $7: 30$ clock positions. The predicted velocity contours of $0.2,0.5,1.0,1.5$, and $2.0 \mathrm{~m} / \mathrm{s}$ along the vertical plane of 7:30 clock position are shown in Figure C.35, while the velocities on the tank bottom are presented in Figure C.36. These figures show that most of the tank area along the jet center line on the tank bottom is above $0.5 \mathrm{~m} / \mathrm{s}$.

To show the potential tank bottom areas (within 4" of the tank bottom) of sludge mobilization for various yield stress values, we constructed the velocity and potential yield stress contours (converted from the predicted velocity, as we discussed) on the tank bottom for the rotating jets. This map was generated by the jet velocity shown in Figure C.36 and rotating them in the tank. The resulting contour maps are shown in Figure 3.8 (and repeated in Figure C.37). This figure shows that about half of the tank bottom experiences a jet velocity greater than $2 \mathrm{~m} / \mathrm{s}$ (or the fluid force exceeding approximately $2,600 \mathrm{~Pa}$ ), and only small corners of the tank farthest from the pump (34 and $32 \mathrm{ft}$ away) have the rotating jet velocity not exceeding $0.2 \mathrm{~m} / \mathrm{s}$ or $0.5 \mathrm{~m} / \mathrm{s}$, or equivalent of 26 and $160 \mathrm{~Pa}$ of the force to act on the sludge bank. Thus if the sludge yield stress is below $26 \mathrm{~Pa}$, then the sludges in all tank bottom areas except those marked less than $0.2 \mathrm{~m} / \mathrm{s}$ (or $26 \mathrm{~Pa}$ ) will be mobilized. As reported in Section 2, SY-102 sludge yield stress was estimated to be $2.7 \mathrm{~Pa}$, much smaller than $26 \mathrm{~Pa}$, based on our rheology measurements.

Once the solids are mobilized, the velocity (or shear stress) must be above some critical values to remain suspended. The critical velocity of water flow needed to erode the sediment in the natural environment varies significantly with the sediment sizes. It varies approximately $0.2 \mathrm{~m} / \mathrm{s}$ for the most easily erodible medium sand (diameter of $0.3 \mathrm{~mm}$ ) to $0.4 \mathrm{~m} / \mathrm{s}$ for the coarse sand (diameter of $2 \mathrm{~mm}$ ), to $1.3 \mathrm{~m} / \mathrm{s}$ for the medium gravel (diameter of $1 \mathrm{~cm}$ ), to $2.5 \mathrm{~m} / \mathrm{s}$ for $1 \mu \mathrm{m}$ clay (Vanoni 1975 ; Simons and Senturk 1977). Some of these values are very approximate, especially the critical velocity to erode clay-sized materials, because of its cohesive nature. The particle densities of most natural sediments are about $2.65 \mathrm{~g} / \mathrm{mL}$, and water density at $20^{\circ} \mathrm{C}$ is $1 \mathrm{~g} / \mathrm{mL}$. Thus the density difference in the natural environment is $1.65 \mathrm{~g} / \mathrm{mL}$. The density difference between Tank SY-102 solids and supernate is about $1 \mathrm{~g} / \mathrm{mL}$. Thus the critical velocity for the tank sludge may be somewhat similar to those in the natural environment. Thus, the tank bottom areas with a velocity of less than $0.2 \mathrm{~m} / \mathrm{s}$ (or corresponding yield stress of $26 \mathrm{~Pa}$ ) in Figures 3.8 and $\mathrm{C} .37$ could be regarded as solid deposition areas. If these solids exhibit cohesiveness, the critical velocity for deposition would be considerably smaller than the critical velocity for erosion (Onishi et al. 1995). Thus even $0.2 \mathrm{~m} / \mathrm{s}$ contour (or 26 $\mathrm{Pa}$ contour) may not be a representative boundary of the potential deposition area.

Figures 3.8 and C.37 show that most areas have velocity (and the corresponding yield stress) above $0.5 \mathrm{~m} / \mathrm{s}$ (and $160 \mathrm{~Pa}$ ), indicating that there are only very small areas (more than $34 \mathrm{ft}$ away from the pump) where the solids may settle on a quasi permanent basis after approximately two hours of jet mixing. The TEMPEST modeling indicates that the rotating jets are judged to be very effective to mix the sludge and supernate in less than two hours, with the bulk of them mixed in 10-20 minutes. Note that the current TEMPEST modeling did not account for cohesiveness, flocculation and break-up of the aggregates, and their effects on the density, viscosity and fall velocity. Additional knowledge and information on solid characteristics, including yield stress, cohesiveness and aggregation, viscosity, and other factors can further improve the accuracy of assessing the potential sludge mobilization and deposition areas. 


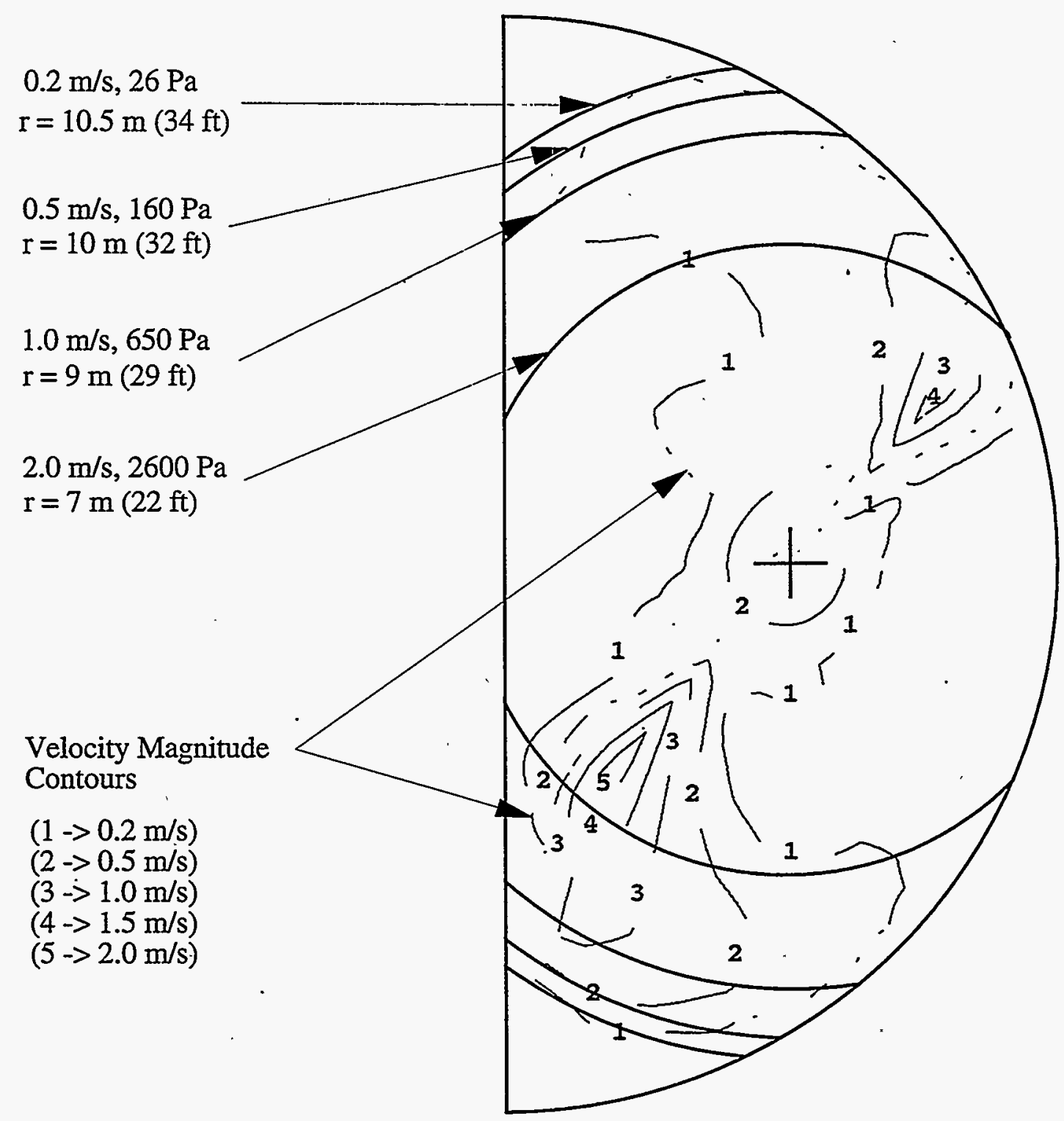

Figure 3.8. Estimated Tank Bottom Areas for Possible Sludge Mobilization at Different Velocity and Yield Stress Values for Case 1 (60 ft/s jets) 


\subsubsection{Case 2: Fully Mixed Case}

TEMPEST simulation results shown in Section 3.3.2 (Case 1) with the sludge initially setting on the tank bottom, indicated that the most of the sludge in the tank will be mobilized less one hour of the continuous pump jet mixing operation. Case 2 discusses how the tank waste moves and accumulates within Tank SY-102 during the subsequent pump jet mixing operation after the tank wastes are completely mixed. To test this operational condition, we assumed that the sludge and supernate are initially completely mixed and uniformly distributed within the tank. With the ratio of sludge to supernate volumes at 1:2, we assigned initial solid volume fractions for the seven solid size fractions to be the values shown in Table 3.7. Otherwise, the modeling conditions are identical to those of Case 1.

We ran TEMPEST for two simulation hours. Predicted velocity and solid fraction distributions in vertical Plane 21 ( 9 o'clock position) and on the tank bottom for Solids 1,5 and 7 at two simulation hours are shown in Figures C.38 through C.43. As comparisons of these figures with corresponding results ( Figs. C.13, C.15, C.17, C.19, C.22, and C.25) of Case 1 (the base case for this study) clearly indicate, the predicted results of the fully mixed case (Case 2 ) and the initially stratified case (Case 1) are very similar after two hours of simulations.

The maximum volume fraction differences over the entire tank and on the tank bottom for Cases 1 and 2 are shown in Table 3.8, indicating very similar results in the two cases. For both Cases 1 and. 2 , very fine solids (Solid 1) are uniformly distributed, but the coarsest solid (Solid 7) shows some effects of settling. As stated previously, although Solid 7 accumulates on the tank bottom more readily, its initial share of the total solids is quite small (only 3\%), as shown in Table 3.1.

Comparisons of the predicted maximum solid volume fractions on the tank bottom for Cases 1 and 2 , are shown in Table 3.9, indicating that these two cases have very similar solid compositions on the tank bottom after two simulation hours. These solid compositions are also very similar to the initial solid compositions assigned for Case 2, as the fully mixed conditions. Segregation factors for all solids at two simulation hours over the initial sludge concentrations on the tank bottom for Case 2 are also very similar to those of Case 1.

As discussed above, the TEMPEST simulation results for Cases 2 are very similar to those of Case 1 at two simulation hours. The comparisons of Cases 1 and 2 support our evaluations of the base case (Case 1) that for the given sludge rheology values assigned for Cases 1 and 2, most of

Table 3.7. Initial Volume Fractions of Seven Solid Size Fractions

\begin{tabular}{|l|c|c|c|c|c|c|c|c||}
\hline & Solid 1 & Solid 2 & Solid 3 & Solid 4 & Solid 5 & Solid 6 & Solid & $\begin{array}{c}\text { Total } \\
\text { Solids }\end{array}$ \\
\hline $\begin{array}{l}\text { Initial Volume } \\
\text { Fraction }\end{array}$ & 0.0123 & 0.0133 & 0.0123 & 0.0297 & 0.0487 & 0.0283 & 0.0067 & 0.151 \\
\hline
\end{tabular}

Table 3.8. Predicted Maximum Variations (\%) of Predicted Volume Fractions over the Entire Tank and on the Tank Bottom at 2 Simulation Hours for Case 2

\begin{tabular}{|l|c|c|c|}
\hline & Solid 1 & Solid 5 & Solid 7 \\
\hline Case 1 (Base Case) & 0.51 & 8.1 & 37 \\
\hline Case 2 (Fully Mixed) & 0.57 & 7.7 & 36 \\
\hline
\end{tabular}


Table 3.9. Comparison of the Predicted Maximum Volume Fractions over the Entire Tank at Two Simulation Hours for Cases 1 and 2

\begin{tabular}{||c|c|c|c|c|c|c|c|c||}
\hline & Solid 1 & Solid 2 & Solid 3 & Solid . 4 & Solid 5 & Solid 6 & Solid & $\begin{array}{c}\text { Total } \\
\text { Solids }\end{array}$ \\
\hline Case 1 & $\mathbf{0 . 0 1 2}$ & 0.013 & 0.012 & 0.029 & 0.054 & 0.033 & 0.0087 & $0: 162$ \\
\hline Case 2 & 0.012 & 0.014 & 0.013 & 0.031 & 0.052 & 0.032 & 0.0093 & 0.163 \\
\hline
\end{tabular}

the sludge currently settled on the tank bottom will be mobilized by the rotating jets located at $20 \mathrm{ft}$ off center, and that its final quasi-state conditions will be achieved within one to two hours.

\subsubsection{Cases 3 and 4: High Viscosity (4,500 Pa-s), Stratified and Fully Mixed Sludge Cases}

Viscosity of the SY-102 sludge varies significantly with strain rate, ranging from approximately 5,000 Pa-s at $0.06 \mathrm{~s}^{-1}$ to $0.1 \mathrm{~Pa}-\mathrm{s}$ at $100 \mathrm{~s}^{-1}$, as discussed in Section 2. To assess the potential jet mixing, and sludge movement and settling under very high viscosity conditions as bounding calculations, we imposed the viscosity variation with the bulk solid concentrations represented in Figure 3.2 (having the maximum viscosity of 4,500 $\mathrm{Pa}$-s), as Cases 3 and 4 conditions. For Case 3 , all the sludge was assumed to stay on the tank bottom initially. For Case 4, the sludge and supernate were assumed to be initially fully mixed and uniformly distributed in the tank. Thus, except for the viscosity values, Case 3 conditions are the same as those of Case 1, and Case 4 conditions are the same as those of Case 2.

We ran the TEMPEST for two simulation hours for these two cases. For Case 3, some of the predicted velocity and solid fraction distributions in vertical Plane 21 (9 o'clock position) and on the tank bottom for Solids 1,5 and 7 at two simulation hours are shown Figures C. 44 through C.49. Corresponding results for the base case (Case 1) are shown in Figures C.13, C.19, C.15, C.22, C.17, and C.25. These figures reveal that, in spite of a large sludge viscosity difference between Cases 1 and 3 , the predicted distributions of the velocity and solid concentrations and the solid accumulation patterns are very similar at two simulated hours.

For Case 4, predicted velocity and Solid 1, 5 and 7 distributions in vertical Plane 21 (9 o'clock position) and on the tank bottom are shown in Figures C.50 through C.55. These results are very similar to corresponding predictions of Cases 1,2 and 3, indicating that 1) two-hour simulation time is sufficiently long for even this high-viscous sludge (Cases 3 and 4 ) to reach the same final quasisteady state conditions and 2) the high viscosity did not measurably change the final conditions from those of the $12 \mathrm{~Pa}-\mathrm{s}$ viscosity cases (Cases 1 and 2).

The maximum volume fraction differences over the entire tank and on the tank bottom for Cases 1,3 , and 4 are shown in Table 3.10, indicating very similar results among these three cases. The com-* parison of the predicted maximum solid volume fractions on the tank bottom for Cases 1,3 , and 4 shown in Table 3.11 also confirms that these three cases have very similar solid compositions and segregation factors on the tank bottom after two simulation hours.

These figures and tables show that the predicted quasi-steady-state final results of Cases 3 and 4, having very high sludge viscosity $(4,500 \mathrm{~Pa}-\mathrm{s})$, are very similar to those of Case 1 having $12 \mathrm{~Pa}-\mathrm{s}$ sludge viscosity. This is because, as the jet mixes the sludge and supernate, the viscosity of the mixture becomes much less than the original sludge viscosity, as Figures 3.1 and 3.2 clearly indicate. Thus, although the jets originally penetrate into and mobilize the sludge more slowly for Case 3 , they eventually mix the most of the sludge and supernate; thus as the jet mixing progresses, the difference in the viscosity of the two cases becomes much smaller; from 4,500 $\mathrm{Pa}$-s for Case 3 and $12 \mathrm{~Pa}$-s for 
Table 3.10. Comparison of Predicted Maximum Variations (\%) of Predicted Volume Fractions over the Entire Tank and on the Tank Bottom at Two Simulation Hours for Cases 1, 3, and 4

\begin{tabular}{||l|c|c|c||}
\hline & Solid 1 & Solid 5 & Solid 7 \\
\hline Case 1: over Entire Tank & 0.51 & 8.1 & 37 \\
\hline Case 3: over Entire Tank & 0.52 & 7.8 & 36 \\
\hline Case 4: over Entire Tank & 0.57 & 7.6 & 35 \\
\hline Case 1: on Tank Bottom & 0.34 & 5.7 & 27 \\
\hline Case 3: on Tank Bottom & 0.34 & 5.4 & 26 \\
\hline Case 4: on Tank Bottom & 0.40 & 5.3 & 25 \\
\hline
\end{tabular}

Table 3.11. Comparison of the Predicted Maximum Volume Fractions on Tank Bottom at Two Simulation Hours for Cases 1, 3 and 4

\begin{tabular}{||l|c|c|c|c|c|c|c|c||}
\hline \hline & Solid 1 & Solid 2 & Solid 3 & Solid 4 & Solid 5 & Solid 6 & Solid 7 & $\begin{array}{c}\text { Total } \\
\text { Solids }\end{array}$ \\
\hline Case 1 & 0.012 & 0.013 & 0.012 & 0.029 & 0.054 & 0.033 & 0.0087 & 0.162 \\
\hline Case 3 & 0.012 & 0.013 & 0.012 & 0.029 & 0.053 & 0.033 & 0.0087 & 0.161 \\
\hline Case 4 & 0.012 & 0.013 & 0.013 & 0.031 & 0.052 & 0.032 & 0.0092 & 0.162 \\
\hline
\end{tabular}

Case 1 to approximately $10-20 \mathrm{cP}$ for both cases. For Case 4 with initially fully mixed conditions, the viscosity of the mixture was already similar to the viscosity of the mixture after Case 1 sludge was almost fully mixed. Thus, it is expected that Case 4 results will be similar to those final quasi steadystate conditions of Case 1 (and, thus also of Case 2).

However, the close evaluations on the velocity and solid distributions for Case 3 show some small differences between Cases 1 and 3. For example, the comparison of Figure C.19 with C.51 shows that the maximum velocity on the tank bottom is slightly greater for Case 3 , due probably to the slightly more viscous jet mixture pulling the bottom flow more than in the Case 1 mixture. The variations of solids, especially the coarsest Solid 7, also show that solid distributions for Case 3 have slightly more. uniform distributions in the tank, including those on the tank bottom, again due to the slightly higher viscosity of the mixture.

However, these velocity and solid distribution differences between Cases 1 and 3 are practically negligible. Thus, even with the assumed high viscosity of the sludge for Cases 3 and 4 , the bulk of the sludge will be mobilized, provided that viscosity varies as shown in Figure 3.2 and that the yield stress is minimum, as the current rheology measurements for SY-102 sludge indicate.

\subsubsection{Cases 5 and. 6: Center-Located Pump with Stratified and Fully Mixed Sludge Cases}

For two cases (Cases 5 and 6), we placed the pump at the center of the tank with two $60-\mathrm{ft} / \mathrm{s}$ jets rotating at $0.5 \mathrm{rpm}$ in opposite directions. We assigned the viscosity variation to be the same as Case 1 (base case), as shown in Figure 3.1. Case 5 assumed the sludge to be initially settled on the tank bottom, while Case 6 assumed the sludge and supernate were initially fully mixed. 
We ran the TEMPEST for two simulation hours for these two cases also. As in the off-center pump cases (Cases 1 through 4), waste distributions reached their quasi-steady state conditions much less than two simulation hours, as evidenced by the fact that Cases 5 and 6 produced basically the same distributions of velocity and solids by two simulation hours. At two simulation hours, the rotating jets are pointing at 3 and 9 o'clock positions. For Case 5, some of the predicted velocity and solid fraction distributions in vertical Plane 21 ( 9 o'clock position) and on the tank bottom for Solids 1,5 and 7 at two simulation hours are shown Figures C.56 through C.61. Predicted distributions of velocity and solid volume fractions at two simulation hours for Case 6 are shown in Figures C.62 through C.67. As seen in these figures, finer solids are much more uniformly distributed throughout the tank than the coarser solids, as expected.

Compared to the two off-center pump cases, the pump located at the tank center mixes wastes somewhat less vigorously, resulting in approximately 30-90\% more nonuniform distributions of the solids within the tank. This is shown in Table 3.12, which compares the maximum volume fraction differences over the entire tank and on the tank bottom for Cases 1,5 , and 6 . Table 3.13 presents the predicted maximum solid volume fractions on the tank bottom for Cases 1,5 , and 6 at two simulation hours.

\subsubsection{Cases 7 Through 10: Reduced Jet Velocity Cases}

We evaluated the potential sludge mixing, movement, and deposition under reduced jet velocities. We reduced the jet velocity to $75 \%$ (or $45 \mathrm{ft} / \mathrm{s}$ ), $50 \%$ (or $30 \mathrm{ft} / \mathrm{s}$ ), and $25 \%$ (or $15 \mathrm{ft} / \mathrm{s}$ ) of the normal $18 \mathrm{~m} / \mathrm{s}(60 \mathrm{ft} / \mathrm{s})$ velocities of the rotating jets. Cases 7,8 and 10 are $75 \%, 50 \%$ and $25 \%$ jet velocity cases with the sludge initially settled on the tank bottom. Case 9 deals with the $50 \%$ jet velocity with the sludge and supernate initially fully mixed and uniformly distributed. Viscosity variations for all these cases were assumed to be represented by that shown in Figure 3.1.

Table 3.12. Comparison of Predicted Maximum Variations (\%) of Predicted Volume Fractions over the Entire Tank and on the Tank Bottom at Two Simulation Hours for Cases 1, 5, and 6

\begin{tabular}{||l|c|c|c||}
\hline & Solid 1 & Solid 5 & Solid 7. \\
\hline Case 1: over Entire Tank & 0.51 & 8.1 & 37 \\
\hline Case 5: over Entire Tank & 0.87 & 11.4 & 49 \\
\hline Case 6: oyer Entire Tank & 0.89 & 11.4 & 49 \\
\hline Case 1: on Tank Bottom & 0.34 & 5.7 & 27 \\
\hline Case 5: on Tank Bottom & 0.61 & 11 & 36 \\
\hline Case 6: on Tank Bottom & 0.82 & 8.2 & 37 \\
\hline
\end{tabular}

Table 3.13. Comparison of the Predicted Maximum Volume Fractions on Tank Bottom at Two Simulation Hours for Cases 1, 5, and 6

\begin{tabular}{|l|c|c|c|c|c|c|c|c||}
\hline & Solid 1 & Solid 2 & Solid 3 & Solid 4 & Solid ' 5 & Solid 6 & Solid 7 & $\begin{array}{c}\text { Total } \\
\text { Solids }\end{array}$ \\
\hline Case 1 & 0.012 & 0.013 & 0.012 & 0.029 & 0.054 & 0.033 & 0.0087 & 0.162 \\
\hline Case 5 & 0.012 & 0.013 & 0.012 & 0.029 & 0.053 & 0.034 & 0.0094 & 0.162 \\
\hline Case 6 & 0.012 & 0.014 & 0.013 & 0.031 & 0.053 & 0.033 & 0.010 & 0.166 \\
\hline
\end{tabular}


As before, the predicted distributions of the solid/supernate mixture reached its final, quasisteady-state well before two simulation hours for all reduced jet velocity cases. Reaching the final state within two hours was also conformed by both Case $8(50 \%$ jet velocity with initially stratified sludge) and Case 9 (50\% jet velocity with fully mixed initial conditions) model results having almost same results, as shown in Figures C.68 and C.69 for Case 8 and Figures C.70 and C.71 for Case 9.

Velocity reductions by up to $75 \%$ did not significantly change finer solid distributions (e.g., Solid 1) from those of Case 1 having $60 \mathrm{ft} / \mathrm{s}$ jet velocity (see Figure C.13), as evidenced by Solid 1 vertical distributions ( 9 o'clock position) at two simulation hours shown in Figures C.72 through C.74 for Cases 7, 8, and 10, respectively. However, the coarser solids, especially Solid 7, did show effects of reduced velocities. Figures C.75 and C.76 present predicted vertical ( 9 o'clock position) and horizontal (tank bottom) for 75\% velocity Case 7. The predicted results of Solid 7 for Case 10 with the $25 \%$ jet velocity are shown in Figures C.77 and C.78. The 50\% jet velocity cases were presented previously. Comparisons of these results with corresponding Solid 7 results for Case 1 (Figures C.13 and C.25) show that reduced jet velocities increase the nonuniformity of the coarse solid distributions vertically, due probably to less suspending/mixing coarser solids in the sludge layer, and increase the solids settling. This increasing nonuniformity on vertical directions and coarse solid volume fraction on the tank bottom are shown in the maximum variations (\%) of predicted volume fractions in Table 3.14, and the maximum volume fractions themselves on the tank bottom (within bottom 4") in Table 3.15.

These solid volume fraction changes are due to the velocity distribution changes. Compare the jet velocity contours of $0.2,0.5,1.0,1.5$ and $2.0 \mathrm{~m} / \mathrm{s}$ in a vertical plane ( 9 o'clock position) at two. simulation hours for Cases 7, 8, and 10, as shown in Figures C.79, C.80, and C.81, respectively. Like the $60 \mathrm{ft} / \mathrm{s}$ jets (Case 1), the $45 \mathrm{ft} / \mathrm{s}$ (Case 7) and $30 \mathrm{ft} / \mathrm{s}$ (Case 8) jets reach the tank center with a velocity greater than $1.0 \mathrm{~m} / \mathrm{s}$. However, unlike $60 \mathrm{ft} / \mathrm{s}$ jets, 45 . and $30 \mathrm{ft} / \mathrm{s}$ jets do not hit the tank bottom with velocities greater than $1.0 \mathrm{~m} / \mathrm{s}$ but fast enough to move the bottom flow toward the tank center along the 9 o'clock position. For the $15 \mathrm{ft} / \mathrm{s}$ jets, the velocity does not even reach $0.2 \mathrm{~m} / \mathrm{s}$ on the tank bottom. Thus when the jet in the modeled tank area collides with an opposing jet located in other half of the tank, some of its jet flow is bent down and moves along the tank bottom toward the pump ( 3 o'clock direction), exhibiting significantly different magnitude and direction of flow movement on the tank bottom.

Table 3.14. Comparison of Maximum Variations (\%) of Predicted Volume Fractions over Entire Tank and Tank Bottom at Two Simulation Hours, Cases 1, 7, 8, 9, 10

\begin{tabular}{|l|c|c|c||}
\hline \multicolumn{1}{|c|}{} & Solid 1 & Solid 5 & Solid 7 \\
\hline Case 1: over Entire Tank & 0.51 & 8.1 & 37 \\
\hline Case 7 (75\% Jets): over Entire Tank. & 0.77 & 11 & 47 \\
\hline Case 8 (50\% Jets): over Entire Tank & 1.2 & 16 & 61 \\
\hline Case 9 (50\% Jets): over Entire Tank & 1.2 & 16. & 61 \\
\hline Case 10 (25\% Jets): over Entire Tank & 2.2 & 27 & 82 \\
\hline Case 1: on Tank Bottom & 0.34 & 5.7 & 27 \\
\hline Case 7 (75\% Jets): on Tank Bottom & 0.60 & 7.8 & 35 \\
\hline Case 8 (50\% Jets): on Tank Bottom & 0.77 & 10 & 43 \\
\hline Case 9 (50\% Jets): on Tank Bottom & 0.81 & 10 & 42 \\
\hline Case 10 (25\% Jets): on Tank Bottom & 0.68 & 8.5 & 33 \\
\hline
\end{tabular}


Table 3.15. Comparison of the Predicted Maximum Volume Fractions on Tank Bottom at Two Simulation Hours for Cases 1, 7, 8, 9, and 10

\begin{tabular}{||c|c|c|c|c|c|c|c|c||}
\hline & Solid 1 & Solid 2 & Solid 3 & Solid 4 & Solid 5 & Solid 6 & Solid 7 & $\begin{array}{c}\text { Total } \\
\text { Solid s }\end{array}$ \\
\hline Case 1 & 0.012 & 0.013 & 0.012 & 0.029 & 0.054 & 0.033 & 0.0087 & 0.162 \\
\hline Case 7 & 0.012 & 0.013 & 0.012 & 0.030 & 0.054 & 0.034 & 0.0096 & 0.165 \\
\hline Case 8 & 0.012 & 0.014 & 0.012 & 0.030 & 0.056 & 0.037 & 0.011 & 0.172 \\
\hline Case 9 & 0.012 & 0.014 & 0.013 & 0.032 & 0.055 & 0.036 & 0.012 & 0.173 \\
\hline Case 10 & 0.012 & 0.014 & 0.013 & 0.032 & 0.060 & 0.042 & 0.015 & 0.187 \\
\hline
\end{tabular}

As for Case 1 (see Figures 3.8 and C.37), contour maps of the velocity and the force acting on the sludge bank are constructed from the predicted velocity distributions for the reduced jet velocity cases to estimate approximate areas of potential sludge mobilization areas. They are shown in Figures 3.9 (Figure C.82), 3.10 (C.83), and 3.11 (C.84) for 45, 30, and $15 \mathrm{ft} / \mathrm{s}$ jet velocity cases, respectively. The 45 and $30 \mathrm{ft} / \mathrm{s}$ jets show only minor differences in these two cases for the potential sludge mobilization areas for 160 and $26 \mathrm{~Pa}$ yield stress conditions. However, these areas are significantly smaller than those for the $60 \mathrm{ft} / \mathrm{s}$ jets (Case-1). For 26-Pa yield stress, jets mobilize 25 and $23 \mathrm{ft}$ from the pump location for Cases 7 and 8, while in the $60 \mathrm{ft} / \mathrm{s}$ case it is $34 \mathrm{ft}$ away. Recall a discussion in Section 2 stating that the SY-102 sludge is estimated to have a very small yield stress (approximately $2.7 \mathrm{~Pa}$ ) based on the current rheology measurements. According to Figure $3.11(\mathrm{C} .84)$, the entire tank bottom (up to 4 " above the bottom) has less than $0.2 \mathrm{~m} / \mathrm{s}$ velocity for the $15 \mathrm{ft} / \mathrm{s}$ jet case, so sludge settling on the entire tank bottom with 26-Pa yield stress may occur. These estimates are approximate, as discussed at the end of Section 3.3.2. 
$0.2 \mathrm{~m} / \mathrm{s}, 26 \mathrm{~Pa}$

$\mathrm{r}=8 \mathrm{~m}(25 \mathrm{ft})$

$0.5 \mathrm{~m} / \mathrm{s}, 160 \mathrm{~Pa}$

$\mathrm{r}=7 \mathrm{~m}(22 \mathrm{ft})$

Velocity Magnitude Contours

$(1 \rightarrow 0.2 \mathrm{~m} / \mathrm{s})$

$(2 \rightarrow 0.5 \mathrm{~m} / \mathrm{s})$

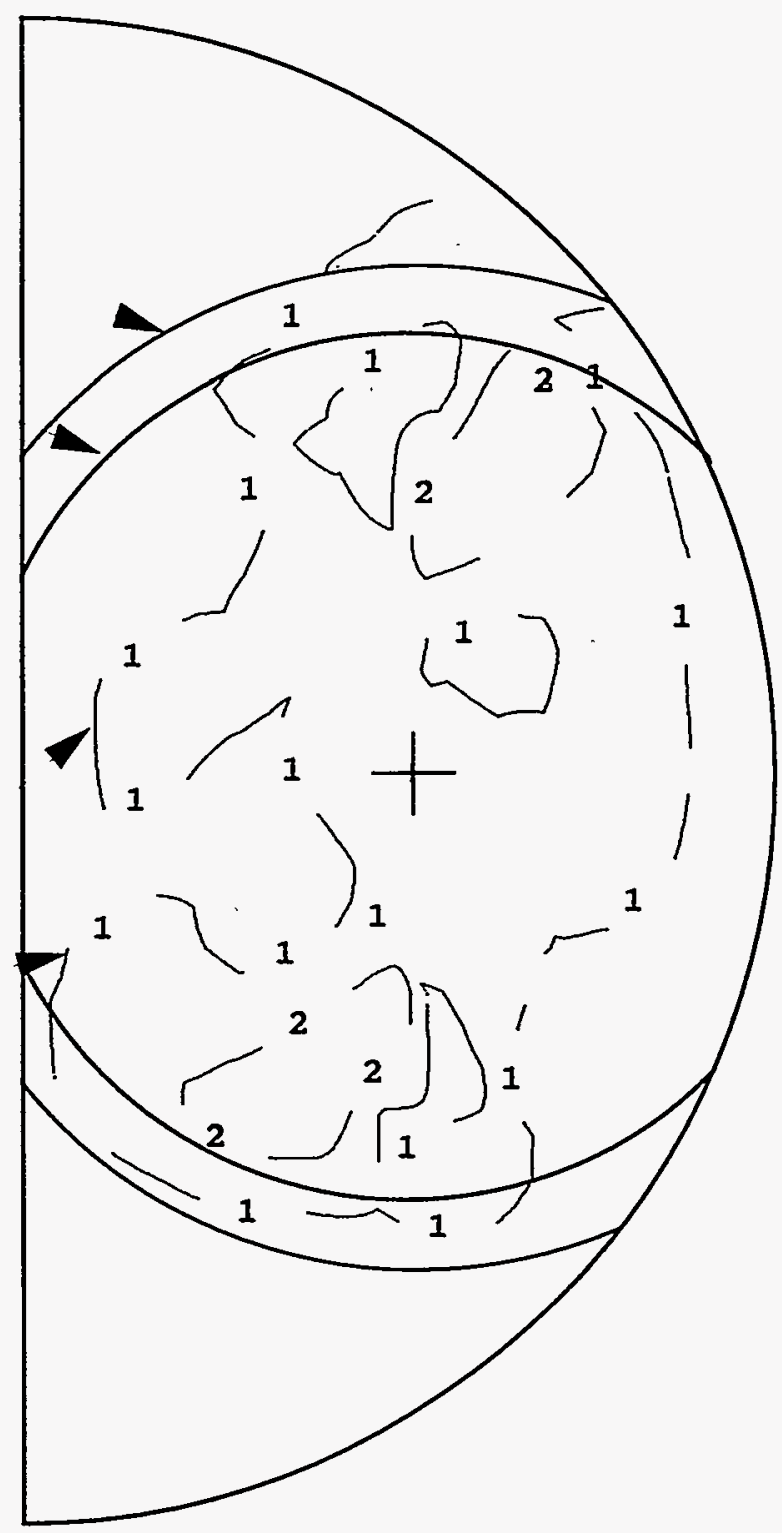

Figure 3.9. Estimated Tank Bottom Areas for Possible Sludge Mobilization at Different Velocity and Yield Stress Values for Case $7 .(45 \mathrm{ft} / \mathrm{s}$ jets) 
$0.2 \mathrm{~m} / \mathrm{s}, 26 \mathrm{~Pa}$

$\mathrm{r}=7 \mathrm{~m}(23 \mathrm{ft})$

$0.5 \mathrm{~m} / \mathrm{s}, 160 \mathrm{~Pa}$

$\mathrm{r}=6 \mathrm{~m}(18 \mathrm{ft})$

Velocity Magnitude Contours

$$
\begin{aligned}
& (1->0.2 \mathrm{~m} / \mathrm{s}) \\
& (2->0.5 \mathrm{~m} / \mathrm{s})
\end{aligned}
$$

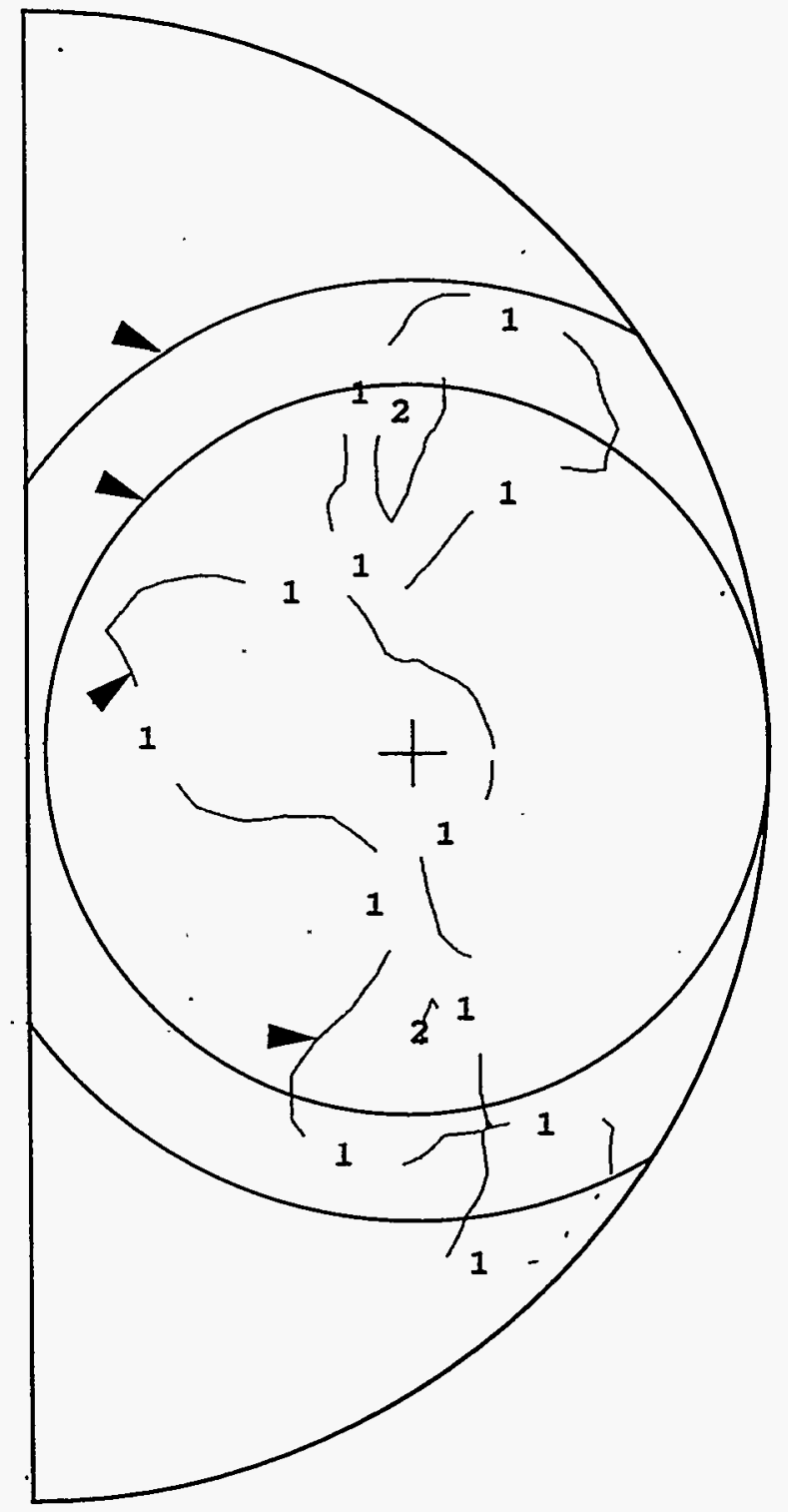

Figure 3.10. Estimated Tank Bottom Areas for Possible Sludge Mobilization at Different Velocity and Yield Stress Values for Case 8 and 9 (30 ft/s jets) 
$0.100 \mathrm{~m} / \mathrm{s}, 6.5 \mathrm{~Pa}$

$\mathrm{r}=9.5 \mathrm{~m}(31 \mathrm{ft})$

$0.125 \mathrm{~m} / \mathrm{s}, 10 \mathrm{~Pa}$

$\mathrm{r}=8 \mathrm{~m}(27 \mathrm{ft})$

$0.150 \mathrm{~m} / \mathrm{s}, 15 \mathrm{~Pa}$

$\mathrm{r}=7 \mathrm{~m}(23 \mathrm{ft})$

$0.175 \mathrm{~m} / \mathrm{s}, 20 \mathrm{~Pa}$

$\mathrm{r}=6 \mathrm{~m}(20 \mathrm{ft})$

$0.200 \mathrm{~m} / \mathrm{s}, 26 \mathrm{~Pa}$

$\mathrm{r}=3 \mathrm{~m}(9 \mathrm{ft})$

Velocity Magnitude

Contours

$(1 \rightarrow 0.100 \mathrm{~m} / \mathrm{s})$
$(2 \rightarrow 0.125 \mathrm{~m} / \mathrm{s})$
$(3 \rightarrow 0.150 \mathrm{~m} / \mathrm{s})$
$(4 \rightarrow 0.175 \mathrm{~m} / \mathrm{s})$
$(5 \rightarrow 0.200 \mathrm{~m} / \mathrm{s})$

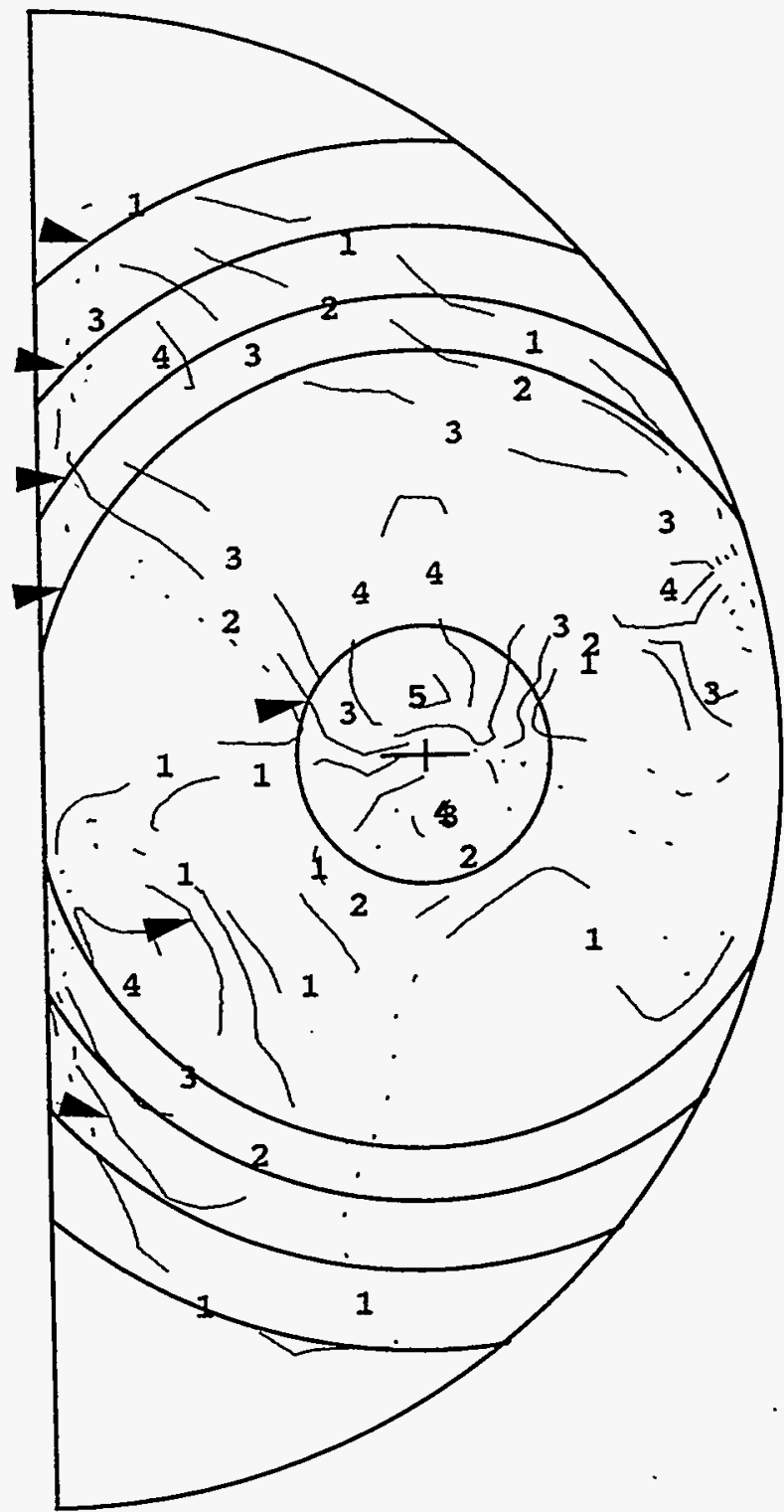

Figure 3.11. Estimated Tank Bottom Areas for Possible Sludge Mobilization at Different Velocity and Yield Stress Values for Case 10 (15 ft/s jets) 
$\because$ 


\subsection{Summary and Conclusions}

This second phase (the detailed assessment) of the SY-102 waste retrieval study was performed to close uncertainty issues on Tank SY-102 waste retrieval and help establish a technical basis for mobilization of the slurry, waste retrieval, and slurry transport. The uncertainties on the preliminary assessment were primarily related to lack of data on waste characteristics and the assumption of the tank being fully mixed. To resolve these issues, this Phase II study 1) identified solid phases of the SY102 waste using TEM; 2) conducted laboratory experiments to measure rheology, zeta potential to evaluate solid agglomeration, and sedimentation; 3) measured particle size distributions of the wastes; and 4) simulated time-varying, three-dimensional, tank pump jet mixing.

The Phase II study results confirm the validity of the assumptions and parameter selections used in the preliminary assessment (Onishi and Hudson 1996) and support its conclusion that the SY-102 waste mixing and heating do not adversely impact the waste retrieval operation. Specific conclusions of this study are described below.

Using TEM, the morphology of the agglomerates was found to be dominated by large-size (2-5 $\mu \mathrm{m} \times 10-30 \mu \mathrm{m}) \mathrm{NaOH}$ rods surrounded by finer primary particles of amorphous silica and iron hydroxide and needle-like $\mathrm{FeO}(\mathrm{OH})$ agglomerates and sharp-edged plate-like $\gamma$ - $\mathrm{Al}_{2} \mathrm{O}_{3}$ particles. The crystalline phases observed included micrometer-sized $\gamma-\mathrm{Al}_{2} \mathrm{O}_{3}$, needle-like $\mathrm{FeO}(\mathrm{OH})$ and $\mathrm{Al}_{2} \mathrm{Si}_{4} \mathrm{O}_{10}$ and to a lesser extent rod-like particles of hydroxylapatite, $\mathrm{Ca}_{5}(\mathrm{PO})_{3}(\mathrm{OH})$ and single crystalline gibbsite, $\mathrm{Al}(\mathrm{OH})_{3}$. The preliminary assessment study (Onishi and Hudson 1996) assumed the all $\mathrm{Al}$ solids to be gibbsite for the chemical reaction modeling. The current TEM identified $\mathrm{Al}$ solids to be $\gamma-\mathrm{Al}_{2} \mathrm{O}_{3}, \mathrm{Al}_{2} \mathrm{Si}_{4} \mathrm{O}_{10}$, and gibbsite. Gibbsite and $\gamma-\mathrm{Al}_{2} \mathrm{O}_{3}$ are chemically similar. Moreover, existence of $\gamma-\mathrm{Al}_{2} \mathrm{O}_{3}$ would slightly increase the amount of water in the solution, as compared with gibbsite. Moreover, since the sludge has a much smaller amount of $\mathrm{Si}$ than the amount of $\mathrm{Al}$, the majority of $\mathrm{Al}$ is not expected to form a solid with $\mathrm{Si}$. Thus, the preliminary assessment with gibbsite may have slightly underestimated the amount of solids being dissolved, resulting in a slightly more conservative estimate (a worse case) for the retrieval operation.

Size analysis results show a volume-averaged mean of $6.435 \pm 1.025 \mu \mathrm{m}(0.422 \pm 0.04 \mu \mathrm{m}$ number-averaged mean) for particles dispersed in deionized water and a volume-averaged mean of $7.245 \pm 1.16 \mu \mathrm{m} \cdot\left(0.866 \pm 0.087 \mu \mathrm{m}\right.$ number-averaged mean) for $1 \underline{\mathrm{M}} \mathrm{NaNO}_{3}$ solution. These results suggest that as the solution becomes more ionic, the size of the aggregates tend to grow. Sonication was shown to break up the larger aggregates.

The zeta potential measurements for the SY-102 waste sample show that the isoelectric point is approximately at $\mathrm{pH}$ of 3.2 suggesting that the silica phase is controlling the surface charge development of the SY-102 waste. This is consistent with the TEM results that show a significant presence of the colloidal amorphous silica in the aggregates.

Rheological measurements of the SY-102 waste show a rheopectic behavior at low shear rates $\left[<O\left(1 \mathrm{~s}^{-1}\right)\right]$ and a thixotropic behavior at high shear rates $\left[\sim O\left(10 \mathrm{~s}^{-1}\right)\right]$. These results suggest that if the slurry is moving at a slow enough rate, aggregation of the primary particles and smaller aggregates, to form larger aggregates, is promoted. In fact, the continuous increase in the viscosity at the lowest shear rate suggests that gelation may be possible, if operated at these shear rates for a sustained period of time. Gelation may be avoided by operating at higher shear rates than $1 \mathrm{~s}^{-1}$. Since the period of jet rotation in SY-102 is expected to be two minutes, gelation is considered not to be important. As the fluid is sheared faster, the rate of disaggregation due to higher shearing stresses increases and balances the rate of agglomeration at shear rate $\sim O\left(1 \mathrm{~s}^{-1}\right)$. At a shear rate of $10 \mathrm{~s}^{-1}$, the disaggregation rate exceeds the agglomeration rate and as a result a thixotropic behavior is observed. And finally, at a high enough rate, the agglomerates have reached an equilibrium size distribution, mainly controlled by the chemistry. 
The undiluted sample was found to have strong pseudoplasticity (shear-thinning) behavior which could be modeled as a power-law fluid, where the consistency factor is $1.5 \mathrm{~Pa} . \mathrm{s}^{\mathrm{n}}$ and behavior index is 0.19 or a Bingham plastic fluid, where the yield stress is $2.7 \mathrm{~Pa}$ and viscosity is $5 \mathrm{mPa}$.. The relative viscosity of the sample was found to decrease when diluted with $\mathrm{NaNO}_{3}$ solution (same ionic strength was maintained) consistent with the empirical model by Chong et al. (1971). Based on the behavior of non-colloidal suspensions we speculate that the reduced viscosity associated with the higher shear rate data for 10 and $100 \mathrm{~s}^{-1}$ are as a result of $5 \%$ and $14 \%$ increase in the maximum packing fractions over the low shear rate limit, respectively. These viscosities were found to be significantly lower than the previous results reported by DiCenso et al. (1995). Thus, the computational modeling results, which are based on the viscosities presented by DiCenso et al., will be conservative.

In the preliminary assessment (Onishi and Hudson 1996), the viscosity of $0.4-1.2 \mathrm{cP}$ for the supernate was used to estimate a critical velocity for solid deposition in the slurry pipeline for temperatures of ranging from 25 to $100^{\circ} \mathrm{C}$. The viscosity of the sludge/supernate mixture was assumed to be $4.8-14 \mathrm{cP}$ to determine an expected pressure drop in the slurry pipeline. The current study estimated that the viscosities of the supernate and the sludge/supernate mixture is approximately 1 and 2 $\mathrm{cP}$, respectively. Thus the preliminary assessment for the pipeline transport was conservative. Thus, the current study supports the preliminary assessment conclusion that is the sludge/supernate mixture will be successfully transferred through RCSTS.

The compressive yield stress was also found to be approximately $80.6 \mathrm{gm} / \mathrm{cm}^{2}(7.9 \mathrm{kPa}$ or 1.2 psi). This value is significantly higher than the shear strength presented by DiCenso et al. (1995). This difference is attributed to DiCenso's approach for measuring the shear strength, which is based on shear vane viscometry. We believe that shear vane results may be grossly over-estimating the shear strength of the waste. Further, the volume fraction of the sludge at a depth of 45 " below the sludgesupernatant layer interface was estimated to be $51 \%$.

The three-dimensional computer code TEMPEST was used to determine whether two pumps with four rotating 60 - $\mathrm{ft}$ jets located $20 \mathrm{ft}$ off-center in Tank SY-102 can sufficiently mix the sludge and supernatant liquid for waste retrieval. The simulation results were also used to evaluate the validity of the previous preliminary assessment's assumption of the completely mixing in SY-102 Tank, and if necessary, to further evaluate the potential chemical equilibrium and kinetic reactions and associated rheology changes by incorporating spatial and temporal distributions of the tank wastes.

TEMPEST simulated the movement of supernate and sludge with seven different solid size fractions under ten different conditions. These ten cases include two sets of viscosity variations, two sets of initial sludge distributions, two different pump locations, and four jet velocities. TEMPEST predicted that the rotating jets would achieve much of the mixing in 10-20 minutes (10-20 sweeps of the rotating jets) in most areas of the tank and that the final, quasi-steady-state is reached in less than two hours, regardless of different viscosity values used in the modeling. Especially the very fine solids (e.g., Solid 1 with 10-20 $\mu \mathrm{m}$ diameters and Solid 2 with $20-30 \mu \mathrm{m}$ diameters) have very uniform distributions within the entire tank. Since the TEMPEST model used more coarser solids reported by DiCenso et al. (1995) than the current measurements of sludge size distributions indicate, the current mixing model results potentially underestimate the degree of the waste mixing.

Thus, the current time-dependent, three-dimensional tank modeling effort confirms the validity of the chemical modeling of the preliminary assessment (Onishi and Hudson 1996) and supports the its conclusion that the SY-102 waste mixing and heating do not adversely impact the. waste retrieval operation. Recall that the current modeling used the DiCenso et al. (1995)'s solid size distribution, which is much more coarser than our measurements indicate, further supporting the validity of the complete mixing`assumption.

Moreover the preliminary assessment concludes that main solids to have dissolution/precipitation reactions were Na-containing solids, especially $\mathrm{NaNO}_{3}(\mathrm{~s})$. Since $\mathrm{NaNO}_{3}(\mathrm{~s})$ is expected to be dissolved or precipitated between 15 minutes and one hour (Onishi and Hudson 1996), the current 
model prediction that the bulk of the mixing will be achieved in 10 minutes to two hours supports the use of the equilibrium chemical modeling performed under the preliminary assessment.

This study also provided estimates of the potential sludge mobilization areas with various yield stress levels. The current TEMPEST modeling did not account for cohesiveness, flocculation and break-up of the aggregates, but since the SY-102 sludge is estimated to have very small yield stress (approximately $2.7 \mathrm{~Pa}$ ), the assumption of no yield stress imposed in this modeling was judged reasonable. Should there actually be a significant yield stress in the sludge, there may be more resistance to sludge mobilization than currently modeled, so it may take longer to mobilize the sludge and may reduce the area of mobilized sludge. Additional knowledge and information on solid characteristics, including yield stress, cohesiveness and aggregation, viscosity, and other factors can further improve the accuracy of assessing the potential sludge mobilization and deposition areas.

The SY-102 model also predicted that the solids are mostly suspended from the tank bottom by the direct hits by the rotating jets and are moved by large scale vertical and horizontal eddies induced in the tank. The suspended solids (especially the coarser solids) in the tank areas not directly hit by the jets settle until they are hit and resuspended again by the rotating jets; thus the solid settling areas keep changing with time over two-minute rotating intervals, except possibly the more stable settling areas in the farthest corners of the tank. Since the sludge is mostly mobilized by the direct hits of the jets, the mixing efficiency is related to the frequency of the jet rotation, i.e., the faster the rotation, the shorter the time required to achieve complete mixing, as long as the jets hit each spot for a reasonable duration during each jet rotation.

The jets with $0.5 \mathrm{rpm}$ are mixing sludge and supernate rapidly and effectively. The finer the solid, the more uniform its distribution is within the tank, as expected. However, even the coarsest solids (Solid 7 having the diameter of $100-175 \mu \mathrm{m}$ ) are reasonably well-mixed. The variations of the predicted solid volume fractions and the maximum volume fractions on tank bottom (within 4" of the tank bottom) under the final, quasi-steady-state conditions for all ten cases are summarized in Tables 4.1 and 4.2. As shown in these tables, when a pump is placed at the center of the tank or the pump jet velocities are reduced from $60 \mathrm{ft} / \mathrm{s}$ to 15,30 , or $45 \mathrm{ft} / \mathrm{s}$, the model predicted less uniformity of the solids within the tank, especially for the coarsest solids.

Table 4.1. Summary of Maximum Variations (\%) of Predicted Volume Fractions over the Entire Tank after Two Simulation Hours for All Ten Cases

\begin{tabular}{||c|c|c|c||}
\hline & Solid 1 & Solid 5 & Solid 7 \\
\hline Case 1 & 0.51 & 8.1 & 37 \\
\hline Case 2 & 0.57 & 7.7 & 36 \\
\hline Case 3 & 0.52 & 7.8 & 36 \\
\hline Case 4 & 0.57 & 7.6 & 35 \\
\hline Case 5 & 0.87 & 11.4 & 49 \\
\hline Case 6 & 0.89 & 11.4 & 49 \\
\hline Case 7 & 0.77 & 11 & 47 \\
\hline Case 8 & 1.2 & 16 & 61 \\
\hline Case 9 & 1.2 & 16 & 61 \\
\hline Case 10 & 2.2 & 27 & 82 \\
\hline
\end{tabular}


Table 4.2. Summary of the Predicted Maximum Volume Fractions on Tank Bottom at Two Simulation Hours after Two Simulation Hours for All Ten Cases

\begin{tabular}{||l|c|c|c|c|c|c|c|c||}
\hline & Solid 1 & Solid 2 & Solid 3 & Solid 4 & Solid 5 & Solid 6 & Solid 7 & Total \\
\hline $\begin{array}{l}\text { Stratified Initial Upper } \\
\text { 38-in. Sludge }\end{array}$ & 0.034 & 0.038 & 0.034 & 0.081 & 0.148 & 0.086 & 0.018 & 0.439 \\
\hline $\begin{array}{l}\text { Stratified Initial Bottom } \\
\text { 8-in. Sludge }\end{array}$ & 0.041 & 0.050 & 0.041 & 0.104 & 0.171 & 0.099 & 0.023 & 0.529 \\
\hline Initially Fully Mixed & 0.0123 & 0.0133 & 0.0123 & 0.0297 & 0.0487 & 0.0283 & 0.0067 & 0.151 \\
\hline Case 1 & 0.012 & 0.013 & 0.012 & 0.029 & 0.054 & 0.033 & 0.0087 & 0.162 \\
\hline Case 2 & 0.012 & 0.014 & 0.013 & 0.031 & 0.052 & 0.032 & 0.0093 & 0.163 \\
\hline Case 3 & 0.012 & 0.013 & 0.012 & 0.029 & 0.053 & 0.033 & 0.0087 & 0.161 \\
\hline Case 4 & 0.012 & 0.013 & 0.013 & 0.031 & 0.052 & 0.032 & 0.0092 & 0.162 \\
\hline Case 5 & 0.012 & 0.013 & 0.012 & 0.029 & 0.053 & 0.034 & 0.0094 & 0.162 \\
\hline Case 6 & 0.012 & 0.014 & 0.013 & 0.031 & 0.053 & 0.033 & 0.010 & 0.166 \\
\hline Case 7 & 0.012 & 0.013 & 0.012 & 0.030 & 0.054 & 0.034 & 0.0096 & 0.165 \\
\hline Case 8 & 0.012 & 0.014 & 0.012 & 0.030 & 0.056 & 0.037 & 0.011 & 0.172 \\
\hline Case 9 & 0.012 & 0.014 & 0.013 & 0.032 & 0.055 & 0.036 & 0.012 & 0.173 \\
\hline Case 10 & 0.012 & 0.014 & 0.013 & 0.032 & 0.060 & 0.042 & 0.015 & 0.187 \\
\hline
\end{tabular}




\subsection{References}

Brinker, C. J. and G. W. Brinker. 1990. Scherer Sol-Gel Science, The Physics and Chemistry of SolGel Processing. Academic Press, New York.

Chang, C. and P. A. Smith. 1996. "Flow-Induced Microstructures in a System of Nuclear Waste Suspensions." Rheologica Acta. 35.

Chang C., and R. L. Powell. 1993. "Dynamic Simulation of Bimodal Suspensions of Hydrodynamically Interacting Spherical Particles.” J. Fluid Mech. Vol. 253, pp. 1-25.

Chong, J. S., E. B. Christiansen, and A. D. Baer. 1971. "Rheology of Concentrated Suspensions." J. Appl. Polymer Sci., 15, pp. 2007-2021.

DiCenso, A. T., L. C. Amato, and W. I. Winters. 1995. Tank Characterization Report for DoubleShell Tank 241-SY-102. WHC-SD-WM-ER-366 Rev. 0, Westinghouse Hanford Company, Richland, Washington.

Felmy, A. R. 1990. GMIN: A Computerized Chemical Equilibrium Model Using a Constrained Minimization of the Gibbs Free Energy. PNL-7281, Pacific Northwest Laboratory, Richland, Washington.

Heckroodt, R. O., and W. Ryan. 1978. Trans. Brit. Ceram. Soc., 77, 177.

LaFemina, J. P. 1995a. Tank Waste Treatment Science: Report for the Third Quarter FY 1995. Pacific Northwest Laboratory, Richland, Washington.

LaFemina, J. P. 1995b. Tank Waste Treatment Science Task Quarterly Report for January-March 1995. PNL-10763, pp. 7.1-7.31. Pacific Northwest Laboratory, Richland, Washington.

Landman, K. A., L. R. White, and R. Buscall. 1988. "The Continuous-Flow Gravity Thickener: Steady State Behavior." AlChE J., No. 34, p. 239.

Liu, J., L. R. Pederson, and L. Q. Wang. 1995. Solid-Phase Characterization in Flammable-GasTank Sludges by Electron Microscopy. PNL-10723, Pacific Northwest Laboratory, Richland, Washington.

Mahoney, L. A., and D. S. Trent. 1995. Correlation Models for Waste Tank Sludge and Slurries. PNL10695, Pacific Northwest National Laboratory, Richland, Washington.

Onishi, Y., H. C. Graber, and D. S. Trent. 1993. "Preliminary Modeling of Wave-Enhanced Sediment and Contaminant Transport in New Bedford Harbor." In Nearshore and Estuarine Cohesive Sediment Transport, AJ Mehta, ed. Vol. 42, Book Series on Coastal and Estuarine Studies, American Geophysical Union, pp. 541-557.

Onishi, Y., H. C. Reid, and D. S. Trent. 1995. Dilution Physics Modeling: Dissolution/Precipitation Chemistry. PNL-10815, Pacific Northwest National Laboratory, Richland, Washington.

Onishi, Y., and J. D. Hudson. 1995. Waste Mixing and Diluent Selection for the Planned Retrieval of Hanford Tank 241-SY-102: A Preliminary Assessment. PNNL-10927, Pacific Northwest National Laboratory, Richland, Washington.

Onishi, Y., H. C. Reid, and D. S. Trent, and J. D. Hudson. 1996. "Tank Waste Modeling with Coupled Chemistry and Hydrothermal Dynamics." Proceedings of the 1996 National Heat Transfer Conference. HTC-Vol. 9, American Nuclear Society Houston, Texas, pp. 262-269. 
Parks, G. A. 1965. "The Isoelectric Point of Solid Oxides, Solid Hydroxides and Aqueous Hydroxyl Complex Systems." Chem. Rev. 62, 2, 177-198.

Rector, D. R. and B. C. Bunker. 1995. "Effect of Colloidal Aggregation on the Sedimentation and Rheological Properties of Tank Waste." TWRSPP-95-027, Pacific Northwest Laboratory, Richland, Washington.

Rodi, W. 1984. "Turbulence Models and Their Application in Hydraulics - a State of the Art Review." Institut fur Hedromechanik, University of Karlsruhe, Germany.

Russel, W. B., D. A. Saville, and W. R. Schowalter. 1989. pp. 456-506. Colloidal Dispersions. Cambridge University Press, New York.

Serne, R. J., G. A. Whyatt, S. V. Mattogold, Y. Onishi, P. M. Doctor, B. N. Bjornstad, M. R. Powell, L. M. Liljegren, J. H. Westsik Jr, N. J. Aimo, K. P. Recknagle, G. R. Golcar, G. R. Holden, D. W. Jeppson, and G. S. Berney. 1996. Fluid Dynamics, Particulate Segregation, Chemical Processes, Natural Ore Analog and Tank Inventory Discussions that Relate to the Potential for Criticality in Hanford Tanks. WHC-SD-WM-TI757 Rev. 0, Westinghouse Hanford Company, Richland, Washington.

Shapiro, A. P., and R. F. Probstein. '1992. "Random Packing of Spheres and Fluidity Limits of Monodisperse and Bidisperse Suspensions." Phys. Rev. Lett. 68, pp. 1422-1425.

Simons, D. B., and F. Senturk. 1977. Sediment Transport Technology. Water Resource Publications, Denver, Colorado.

Trent, D. S., and L. L. Eyler. 1994. TEMPEST: A Computer Program for Three-Dimensional Time Dependent Computational Fluid Dynamics. PNL-8857 Vol. 1, Version T, Mod 2, Pacific Northwest Laboratory, Richland, Washington.

Vanoni, V. A., ed. 1975. Sedimentation Engineering. ASCE Manuals and Reports on Engineering Practice. ASCE. New York.

Whyatt, G. A., R. J. Serne, S. V. Mattogold, Y Onishi, M. R. Powell, J. H. Westsik,Jr, L. M. Liljegren, G. R. Golcar, K. P. Recknagle, P. M. Doctor, V. G. Zhirnov, J. Dixon, D. W. Jeppson, and G. S. Berney. 1996. The Potential for Criticality in Hanford Tanks Resulting from Retrieval of Tank Wastes. PNNL-11304, Pacific Northwest National Laboratory, Richland, Washington. 
Appendix A

Electron Microscopy Results 



\section{Appendix A}

\section{Electron Microscopy Results}

The transmission electron microscopy (TEM) samples were prepared by dispersing a drop of the SY-102 waste slurry on the TEM copper grids covered with carbon films. This work was performed on a JOEL 1200EX analytical TEM equipped a Tracor TN 5500 X-ray electron energy-dispersive spectrometer (EDS) and operated at $120 \mathrm{kV}$. The microstructural characterization and chemical analysis were carried out by the following procedures:

1. The morphology, distribution, and sizes of particles were evaluated by electron imaging. A lowmagnification TEM micrograph of the sample reveals different agglomerates, as shown in Figure A.1. Most of the morphology is dominated by the large-size $(2-5 \mu \mathrm{m} \times 10-30 \mu \mathrm{m}) \mathrm{NaOH}$ rods, which are accompanied by smaller particles with different contrasts, sizes, and shapes. The large-size $\mathrm{NaOH}$ rods are not stable under the electron beam and change contrast, size, and shape in seconds. Figure A.2 is an enlargement of the central part of the image in Figure A.1, showing a mm-sized sharp-edged $\dot{\gamma}-\mathrm{Al}_{2} \mathrm{O}_{3}$ particle surrounded by dark contrast amorphous $\mathrm{FeO}(\mathrm{OH})$ agglomerates containing fine particles, also seen in Figure A.3. There are also needle-like $\mathrm{FeO}(\mathrm{OH})$ particles along with a light contrast sharp-edged $\gamma-\mathrm{Al}_{2} \mathrm{O}_{3}$ particle in Figure A.3. Figure A.4 shows the dark contrast irregular U-containing agglomerates attached to the end of the $\mathrm{NaOH}$ rod in Figure A.1. An $\mathrm{Al}_{2} \mathrm{Si}_{4} \mathrm{O}_{10}$ particle with different contrast on the surface in Figure A.5 and sharp-edged rectangular $(300 \mathrm{~nm} \times 800 \mathrm{~nm})$. Al/Cr-containing particle in Figure A.6 are also observed.

2. The chemical composition of the particles was identified by EDS: The overall EDS (Figure A.7) shows that $\mathrm{SY}-102$ waste is mostly composed of $\mathrm{Na}$ plus $\mathrm{Si}, \mathrm{Al}, \mathrm{Fe}, \mathrm{Cr}$, and $\mathrm{U}$. The large rods in Figure A.1 are Na-containing materials according to the corresponding EDS (Figure A.8). The light contrast agglomerates at the bottom of Figure A.2 are amorphous Si-rich materials (Figure A.9). Figure A.2 shows a micrometer-sized sharp-edged Al (Figure A.10) particle surrounded by amorphous Fe-containing materials (right profile on Figure A.11). The light contrast plate in Figure A.3 is also Al-rich, but the needle-like crystallites with length less than $1 \mu \mathrm{m}$ are Fe-rich (left profile on Figure A.11). The U-containing materials are amorphous (Figure A.12), such as the dark contrast irregular agglomerates attached to the end of the rod shown in Figure A.4. There is no plutonium detected. The particle with different contrast on the surface in Figure A.5 is Al- and Si-rich (Figure A.13). The sharp-edged rectangular particle in Figure A.6 is Al- and Cr-rich, according to the EDS (Figure A.14). Ca is always associated with $\mathrm{P}$ in small rod-like particles (results not shown).

3. The crystal structures of the particles were studied by electron diffraction pattern. The $\mathrm{NaOH}$ rods are too thick to give any diffraction pattern, but multi-crystalline pattern could be observed after exposure to the electron beams from which $\mathrm{NaOH}$ could be identified. The light contrast silica in Figure A.2, the dark contrast iron hydroxide in Figure A.3, and U-containing agglomerates in Figure A.4 are all amorphous (i.e., no diffraction was observed). Single crystalline diffraction patterns were recorded from sharp-edged $\gamma-\mathrm{Al}_{2} \mathrm{O}_{3}$ particles in Figure A.2 and A.3 (Figure A.15), the needle-like $\mathrm{FeO}(\mathrm{OH})$ particles in Figure A.3 (Figure A.16), and the $\mathrm{Al}_{2} \mathrm{Si}_{4} \mathrm{O}_{10}$ particles in Figure A.5 (Figure A.17).

For phase identification, the EDS and the corresponding diffraction pattern were compared with JCPDS-EDD Data Base published by JCPDS International Center for Diffraction Data. The following results were found:

- The multi-crystalline Na phase matches sodium hydroxide hydrate, $\mathrm{Na}(\mathrm{OH}) ! 4 \mathrm{H}_{2} \mathrm{O}$. 
- The single crystalline $\mathrm{Al}$ plates reveal a $\gamma$ aluminum oxide phase, $\gamma-\mathrm{Al}_{2} \mathrm{O}_{3}$.

- The $\mathrm{Al}$ and Si particle with different contrast on the surface is identified as aluminum silicate, $\mathrm{A}_{2} \mathrm{Si}_{4} \mathrm{O}_{10}$.

- The needle-like Fe crystallites are one of the iron oxide hydroxide crystal formation known as akaganeite, $\mathrm{M}(\mathrm{FeO}(\mathrm{OH}))$.

- The other minor crystalline phases observed include: a) small rod-like particles of hydroxylapatite, $\mathrm{Ca}_{5}(\mathrm{PO})_{3}(\mathrm{OH})$, and b) single crystalline gibbsite, $\mathrm{Al}(\mathrm{OH})_{3}$.

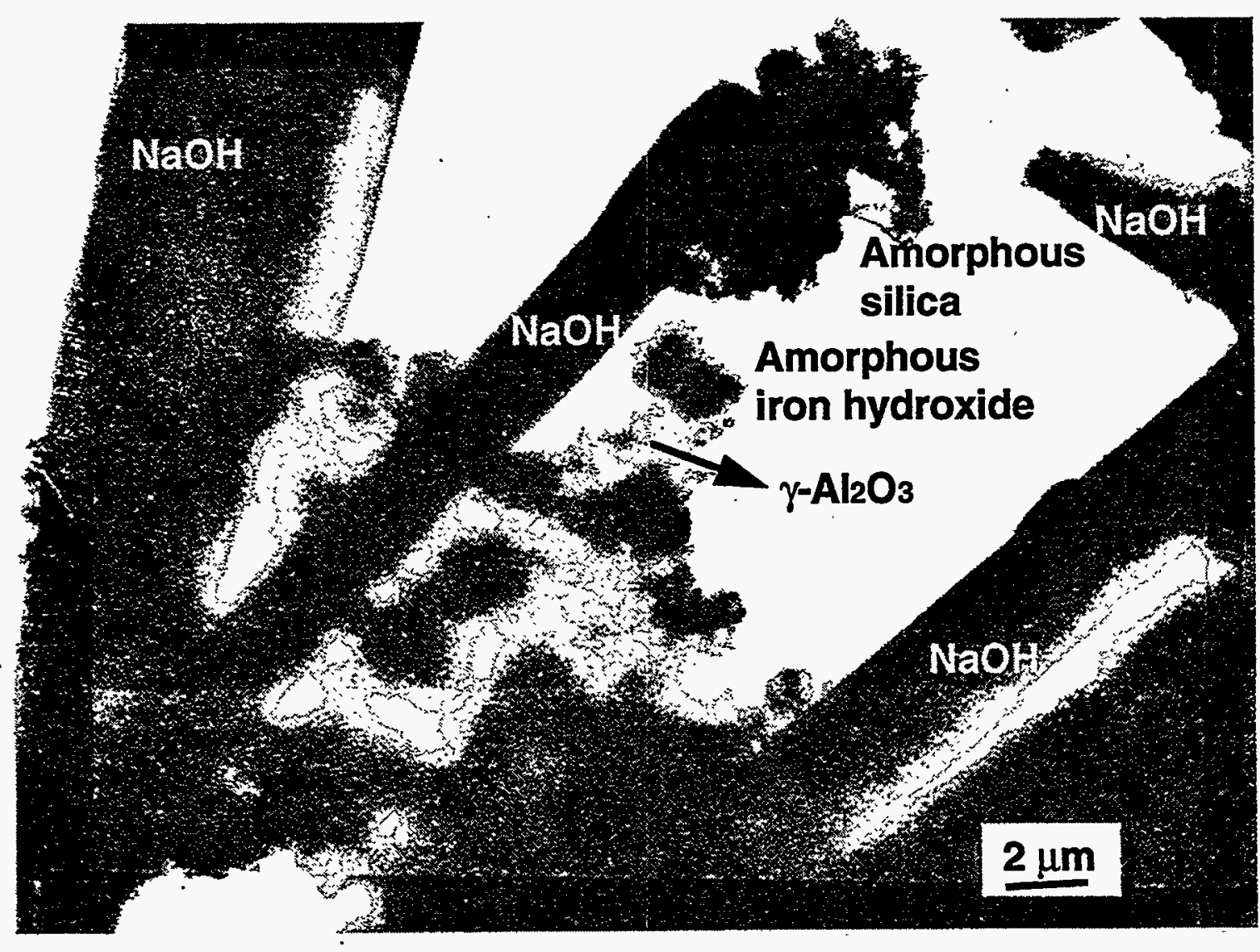

Figure A.1. A Low-Magnification TEM Image of SY-102 Waste Sample (2000X). This image shows the general morphology of soluble and insoluble particles. The large rod-like particles are sodium hydroxide (salt); other phases include crystalline alumina, iron hydroxide, and amorphous iron hydroxide and silica. 


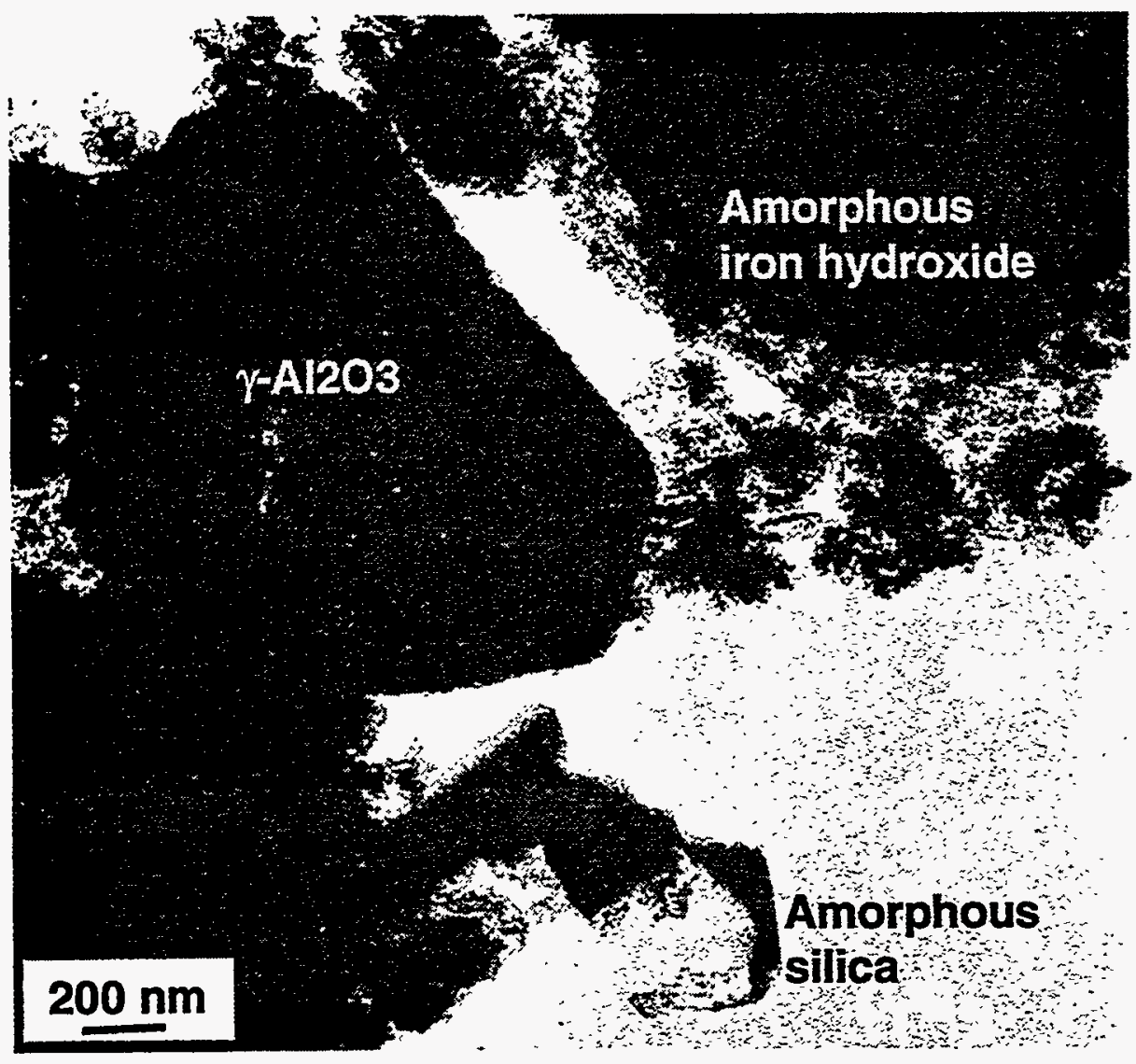

Figure A.2. TEM Image of $\mathrm{g}-\mathrm{Al}_{2} \mathrm{O}_{3}$, Amorphous Silica, and Amorphous Iron Hydroxide Agglomerates 


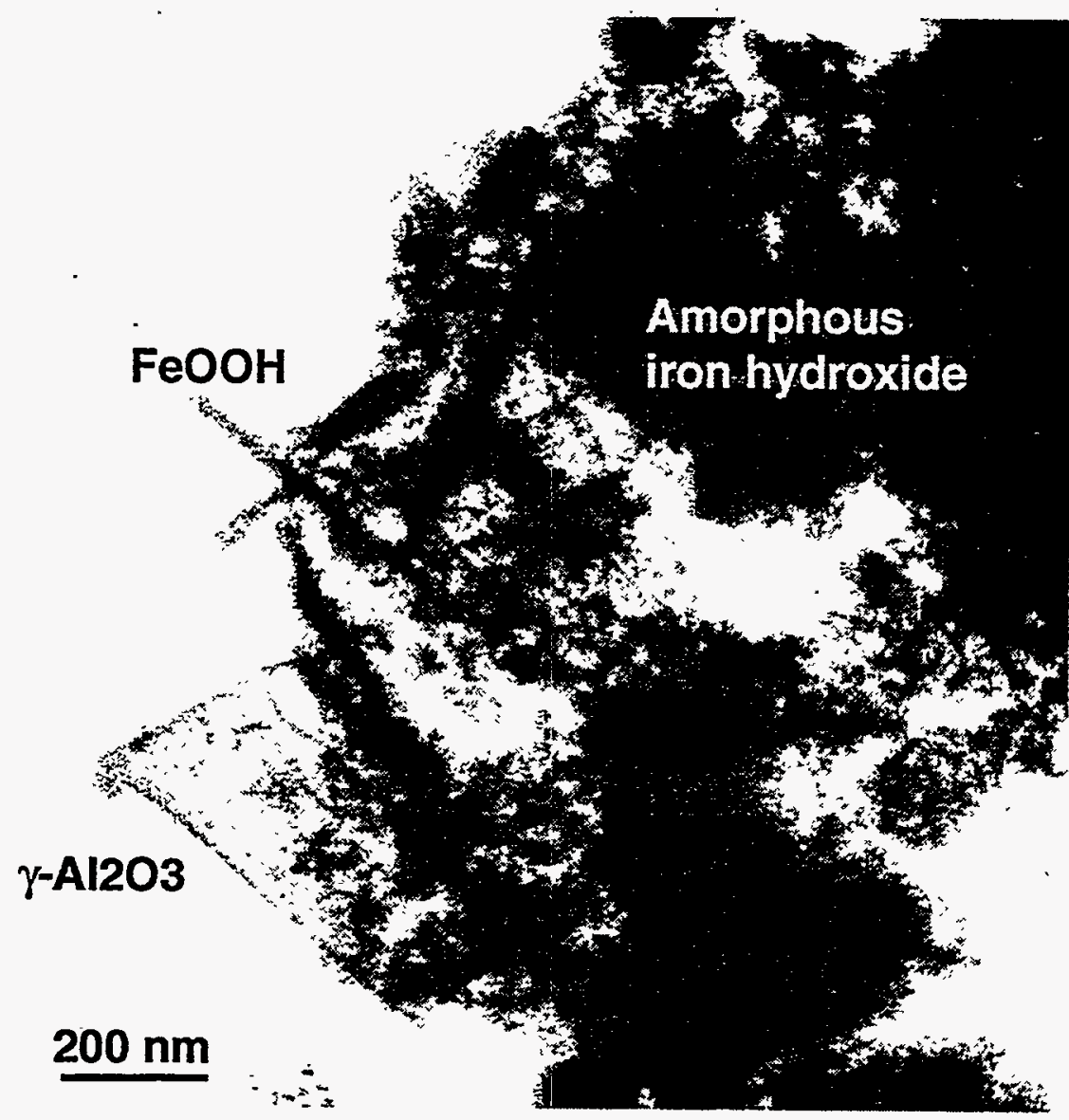

Figure A.3. TEM Image of Rod-Like $\mathrm{FeOOH}, \mathrm{g}-\mathrm{Al}_{2} \mathrm{O}_{3}$, and Amorphous Iron Hydroxide Agglomerates 


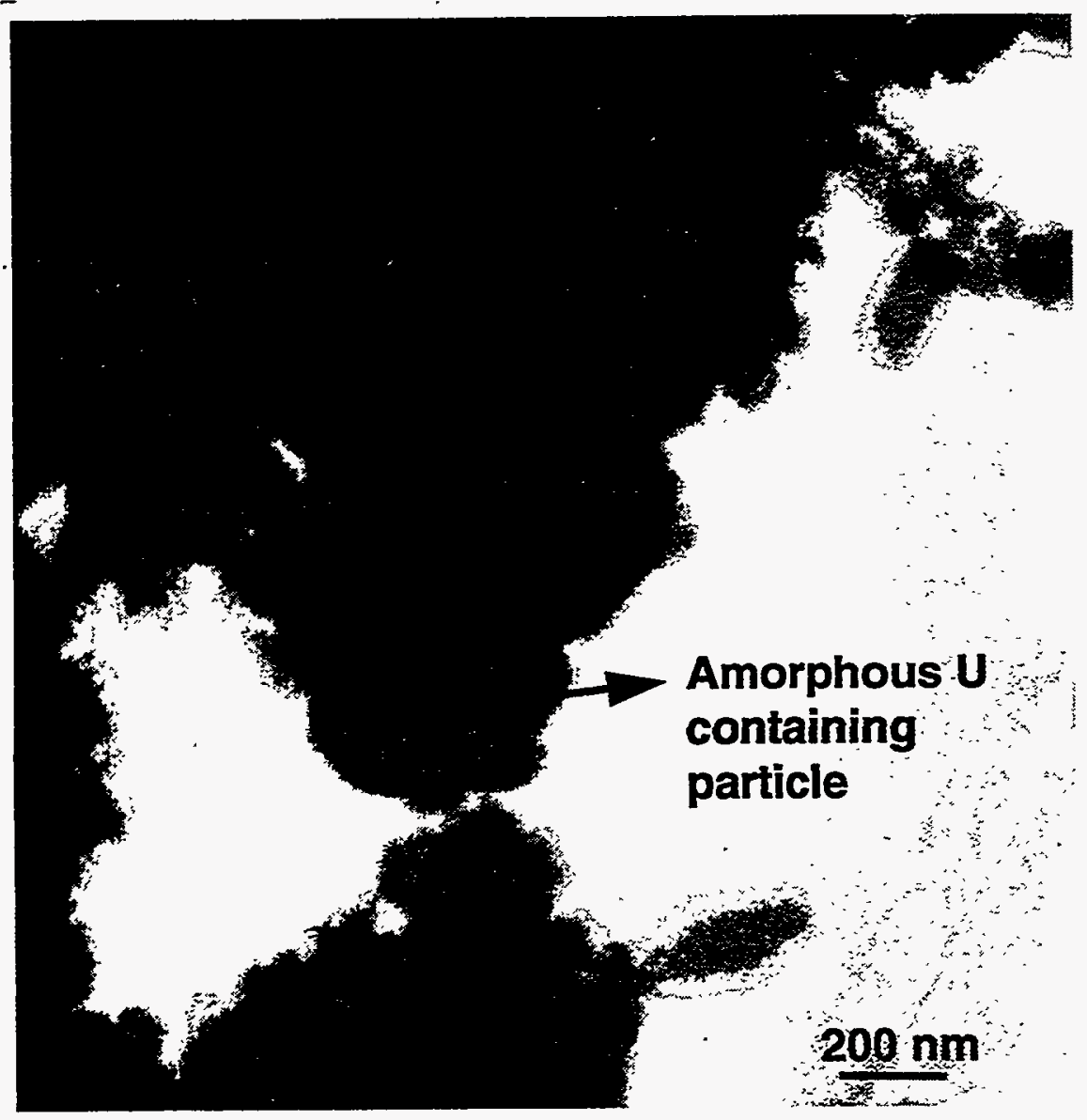

Figure A.4. TEM Image of Amorphous U-Containing Particles 


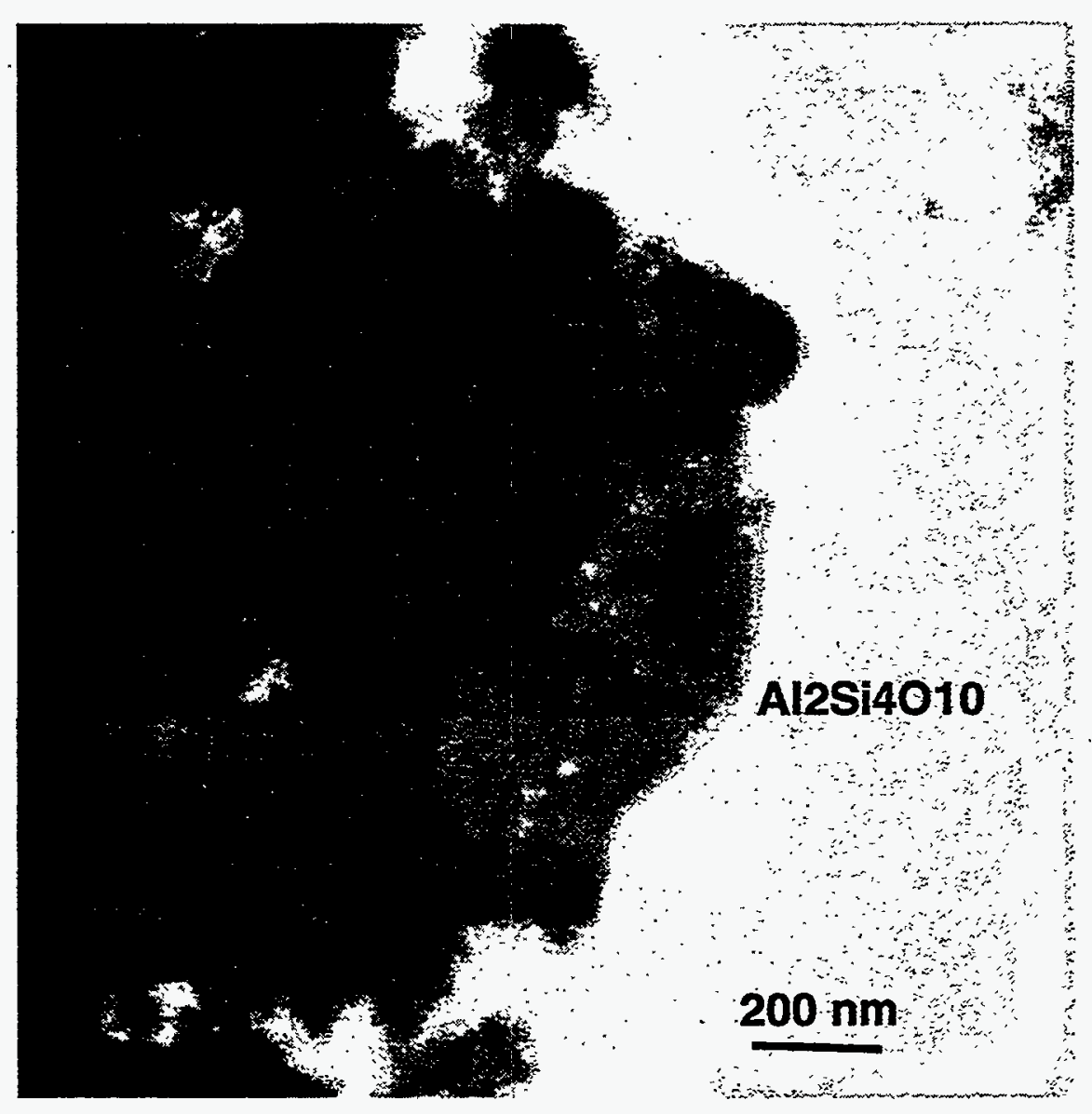

Figure A.5. TEM Image of Aluminosilicate, $\mathrm{Al}_{2} \mathrm{Si}_{4} \mathrm{O}_{10}$ 


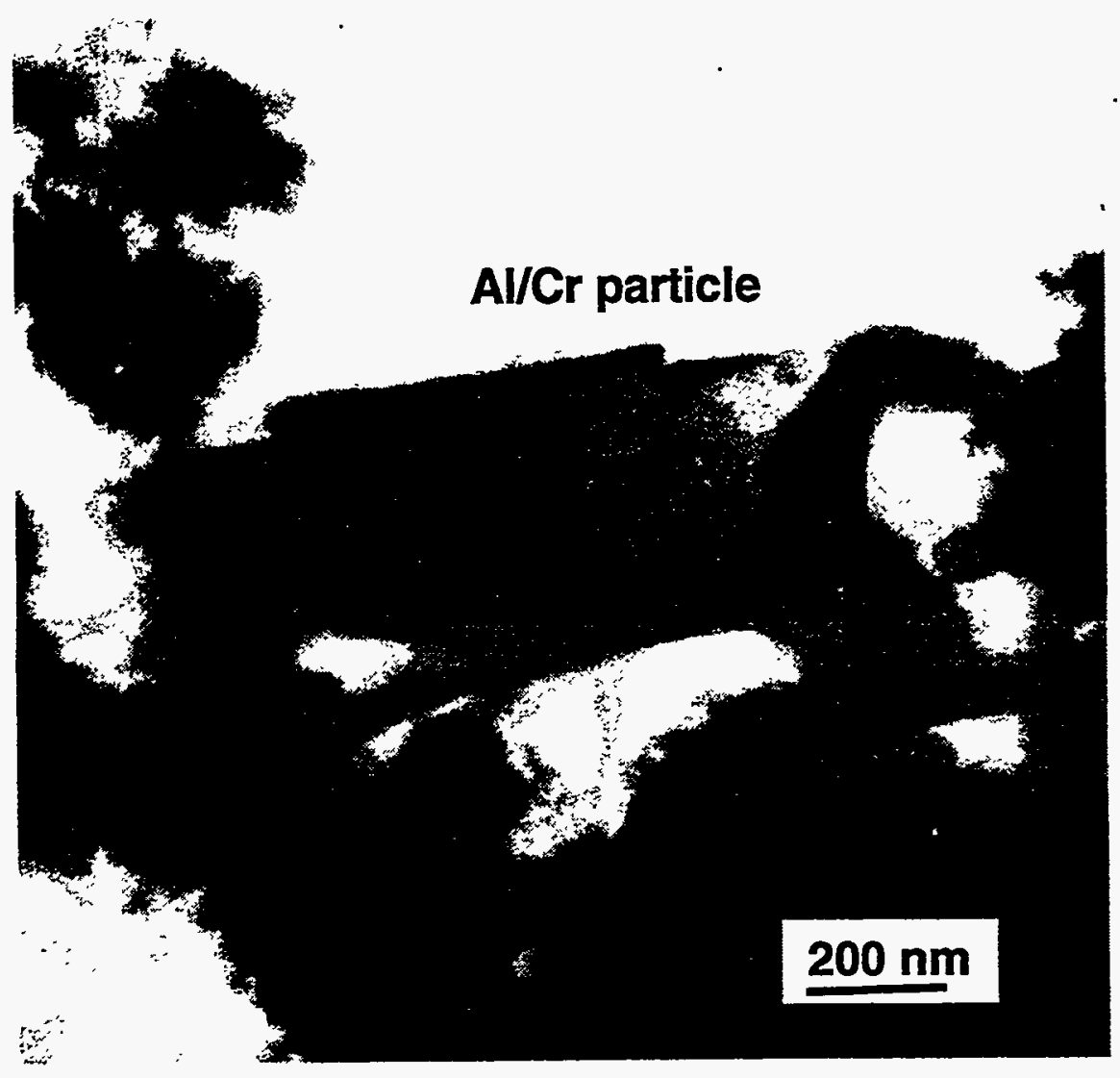

Figure A.6. TEM Image of Particle Containing Both $\mathrm{Al}$ and $\mathrm{Cr}$. The crystalline structure has not been determined. 


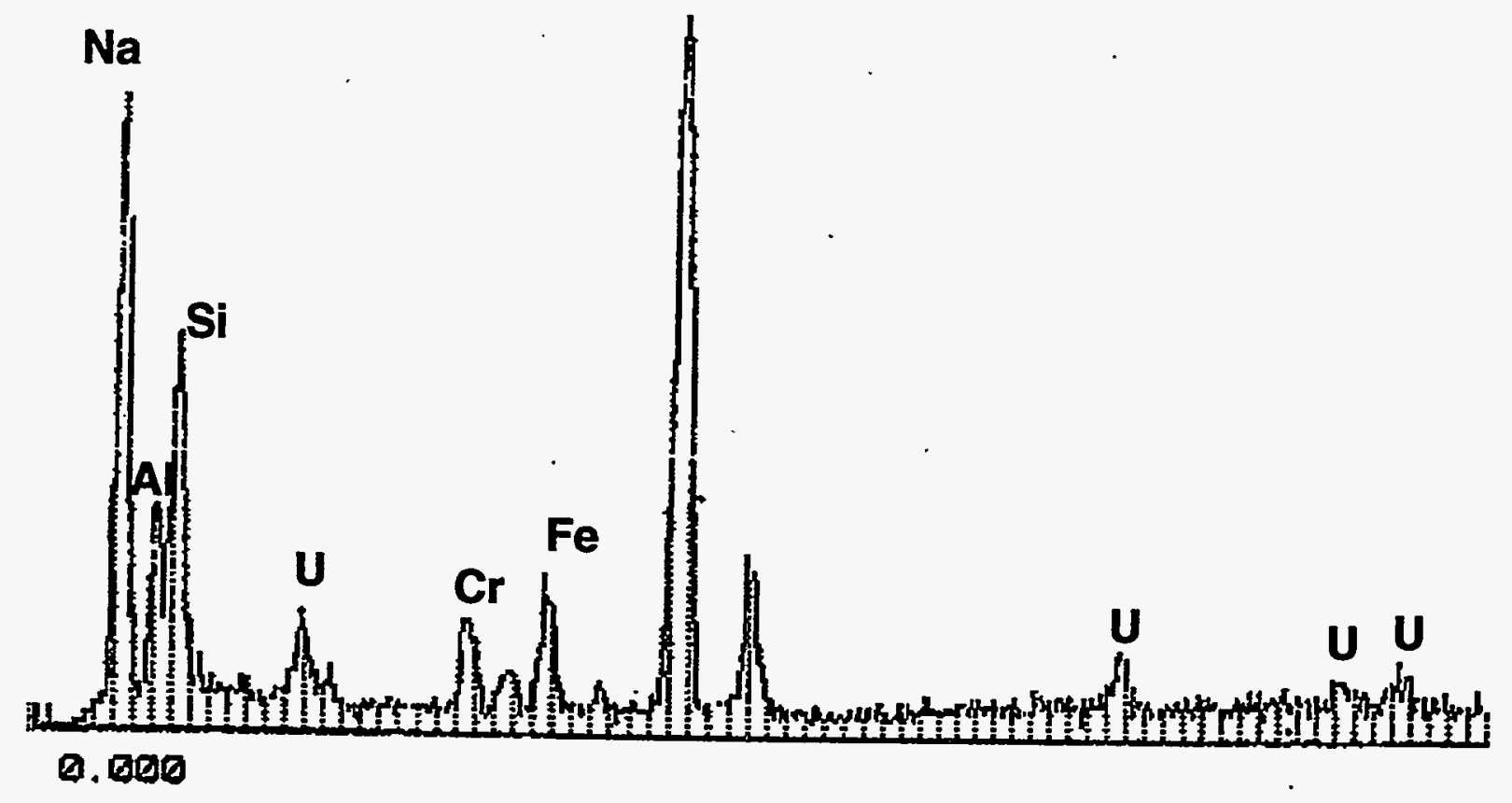

Figure A.7. EDS Spectrum of Figure A.1. This spectrum shows that the general composition is rich in $\mathrm{Na}, \mathrm{Al}, \mathrm{Si}, \mathrm{Fe}, \mathrm{Cr}$, and $\mathrm{U}$

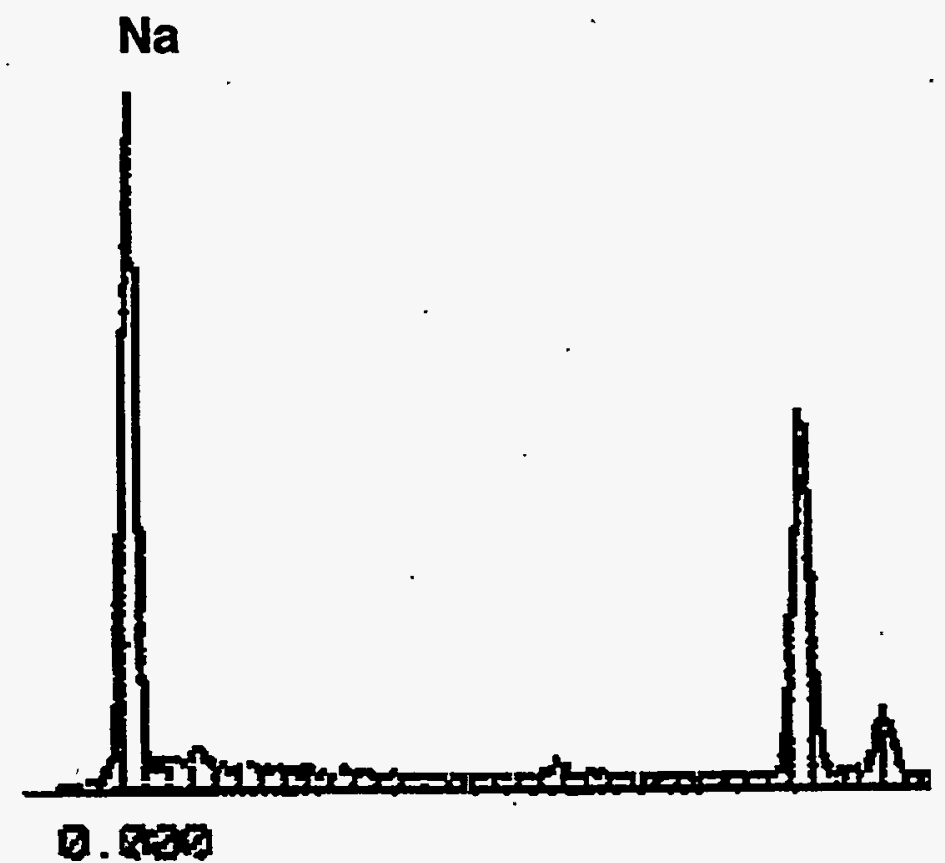

Figure A.8. EDS Spectrum of $\mathrm{NaOH}$ Rod-Like Particles in Figure A.1 


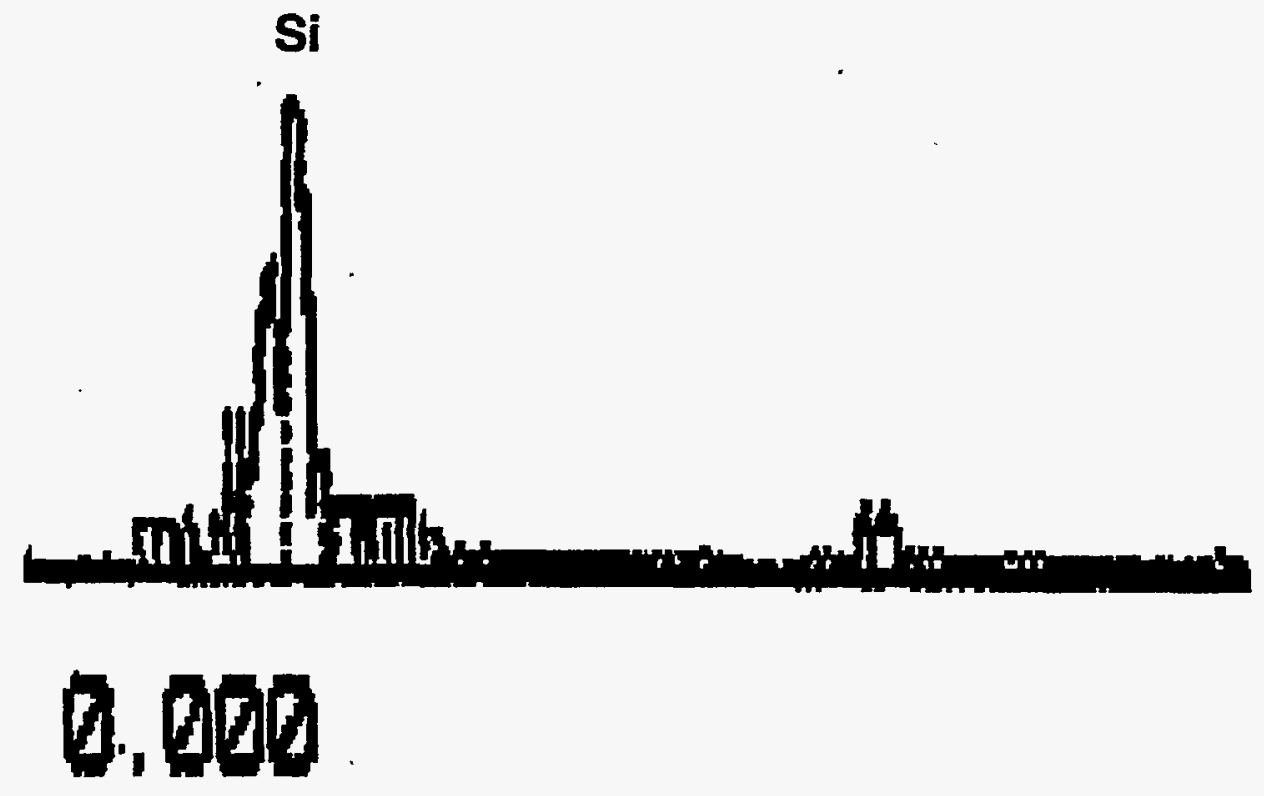

Figure A.9. EDS Spectrum of Amorphous Silica Particles in Figures A.1 and A.2

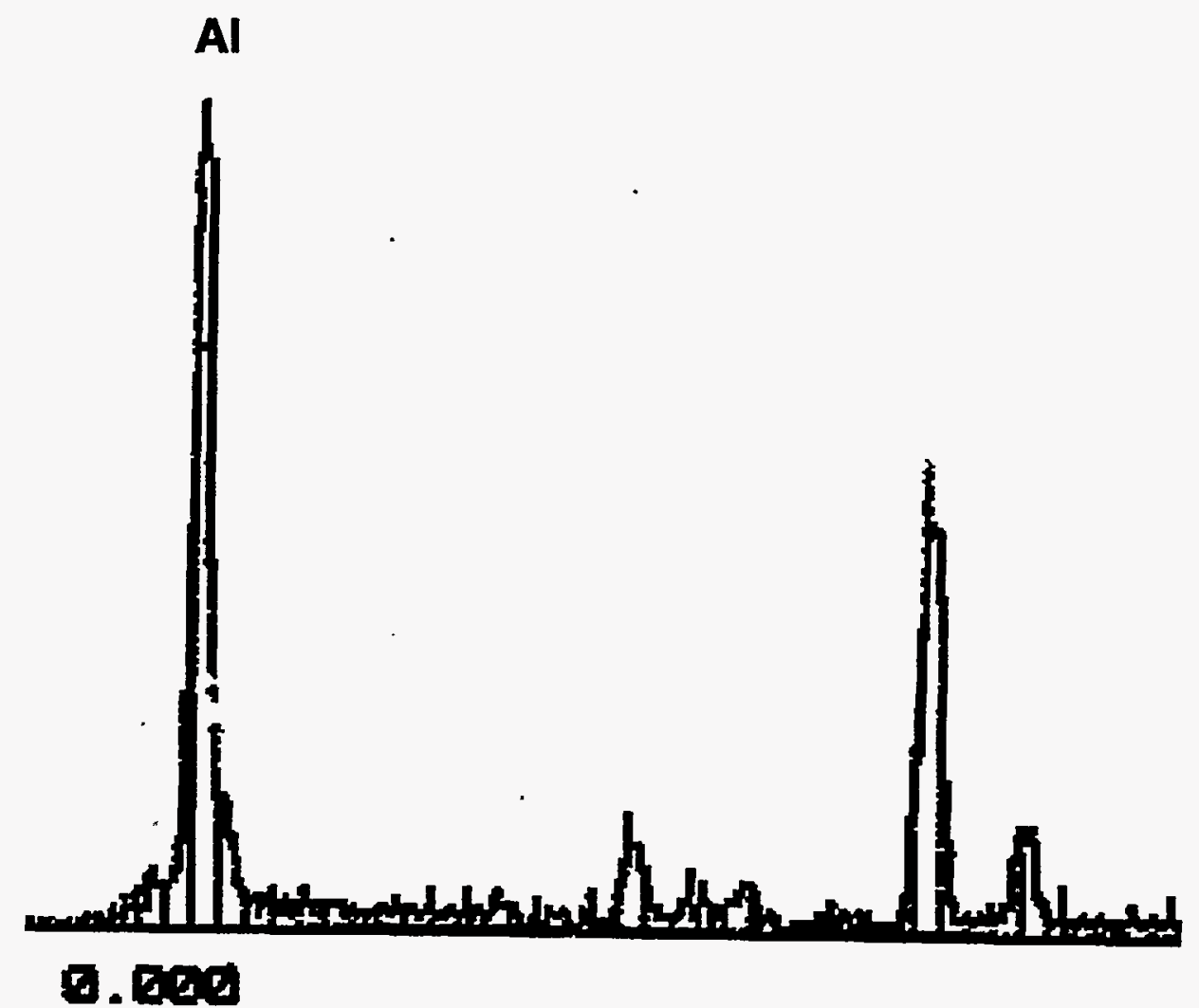

Figure A.10. EDS Spectrum of $\mathrm{g}-\mathrm{Al}_{2} \mathrm{O}_{3}$ as Shown in Figures A.2 and A.3 


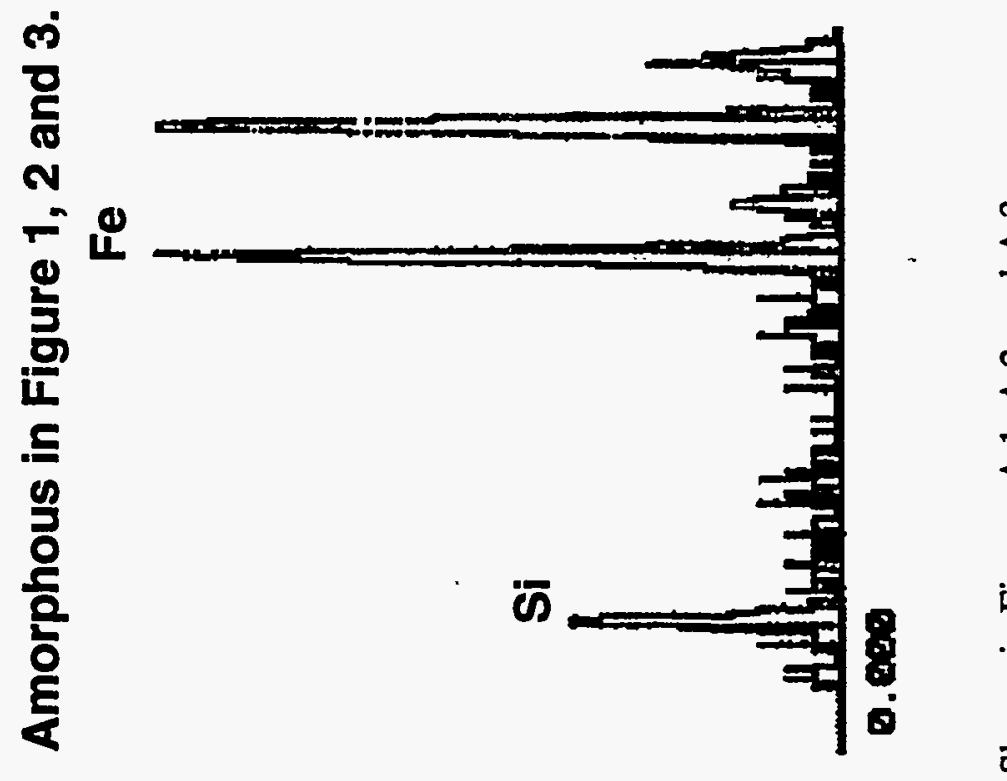

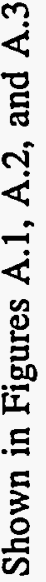

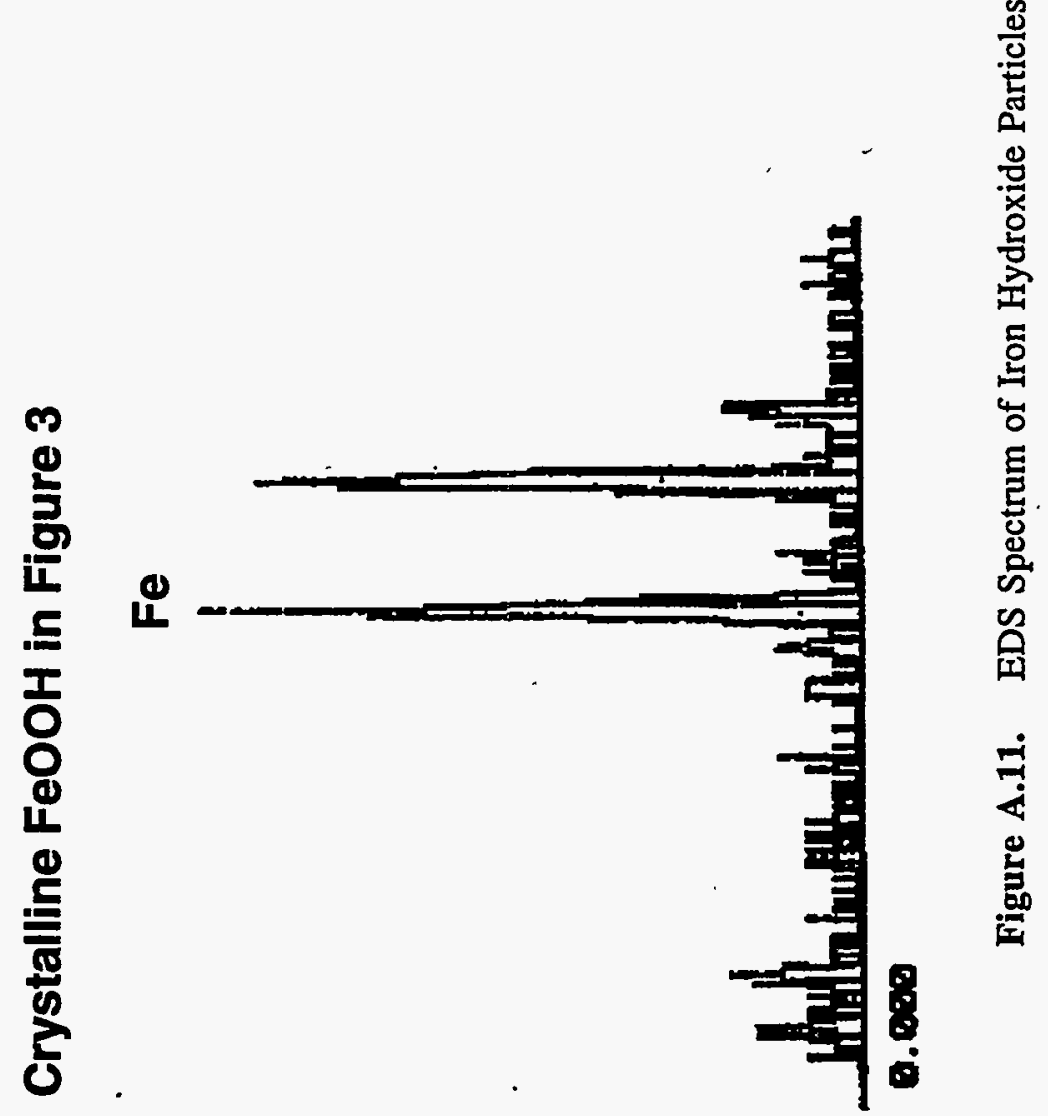

A. 10 


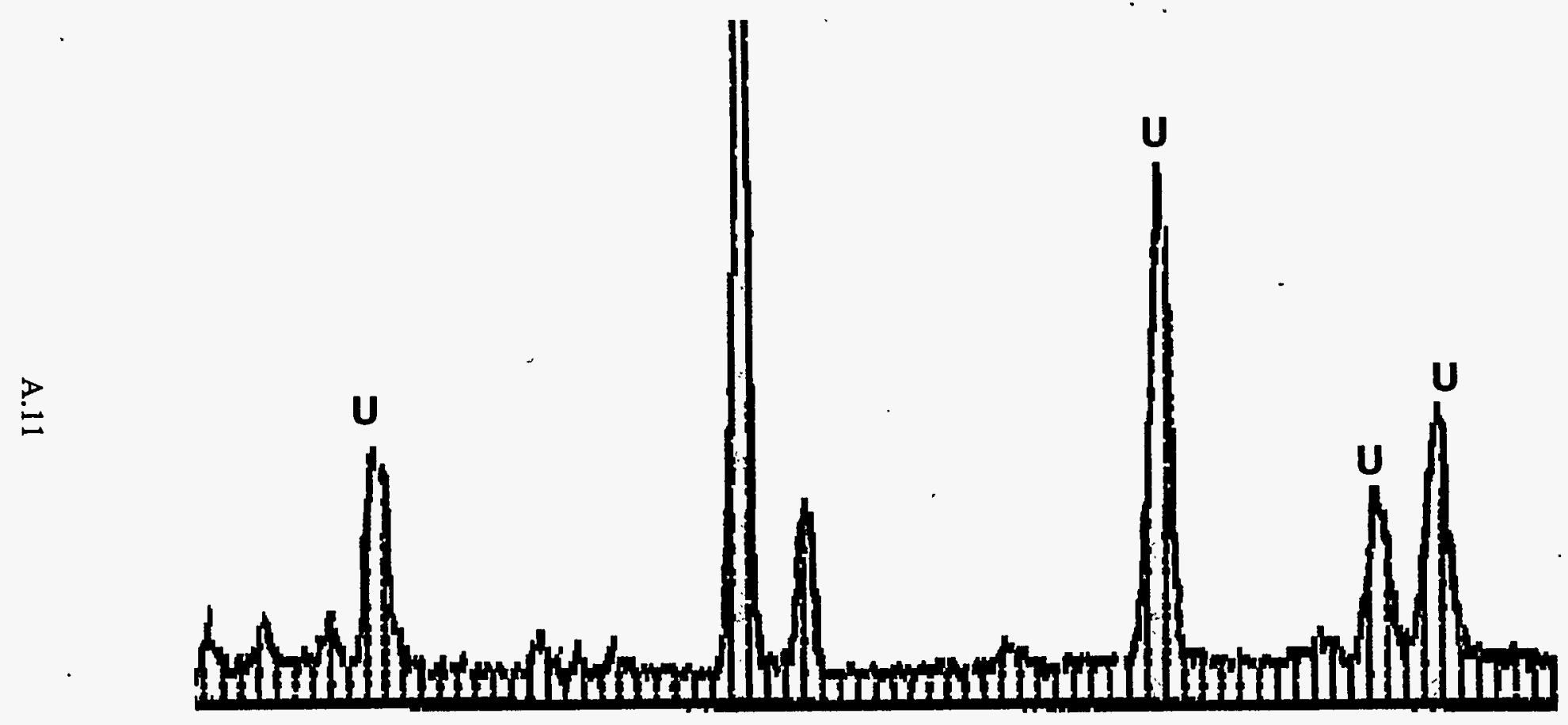

Figure A.12. EDS Spectrum of Amorphous U-Containing Particles 


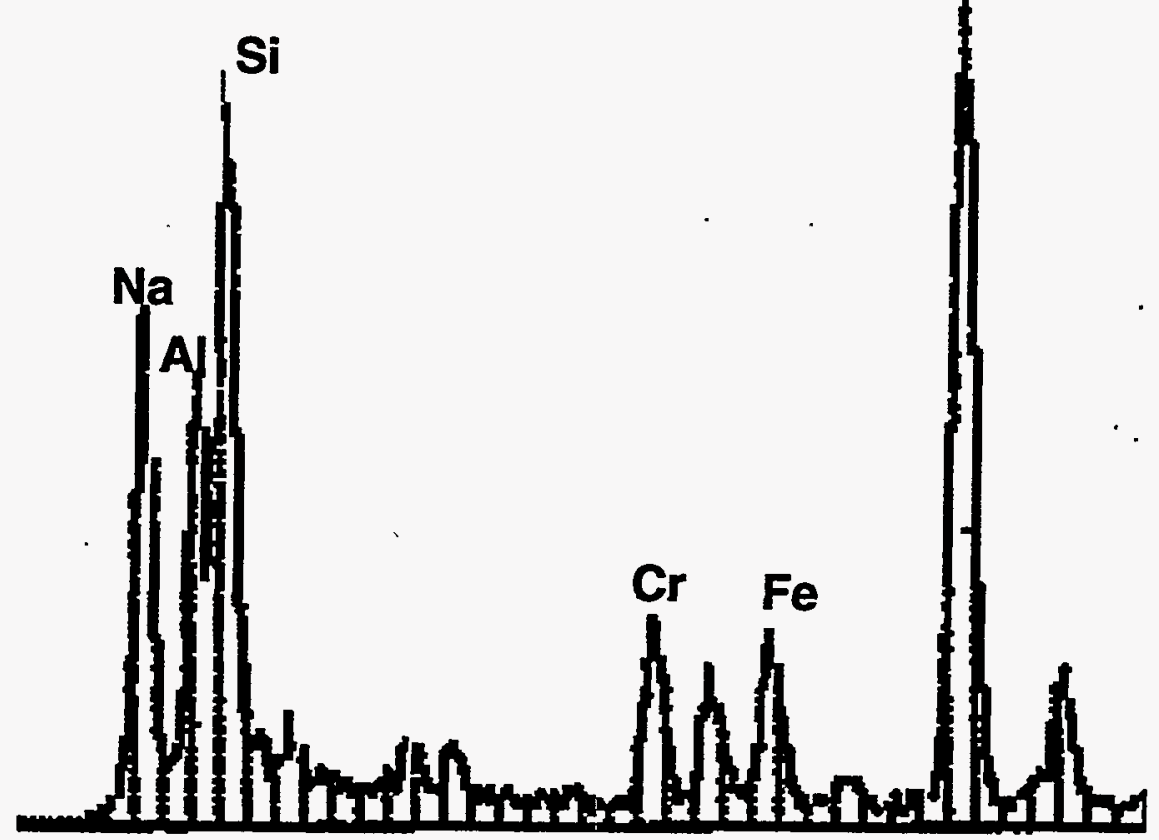

E. $2+r_{1}$

Figure A.13. EDS Spectrum of Aluminosilicate Particles in Figure A.5

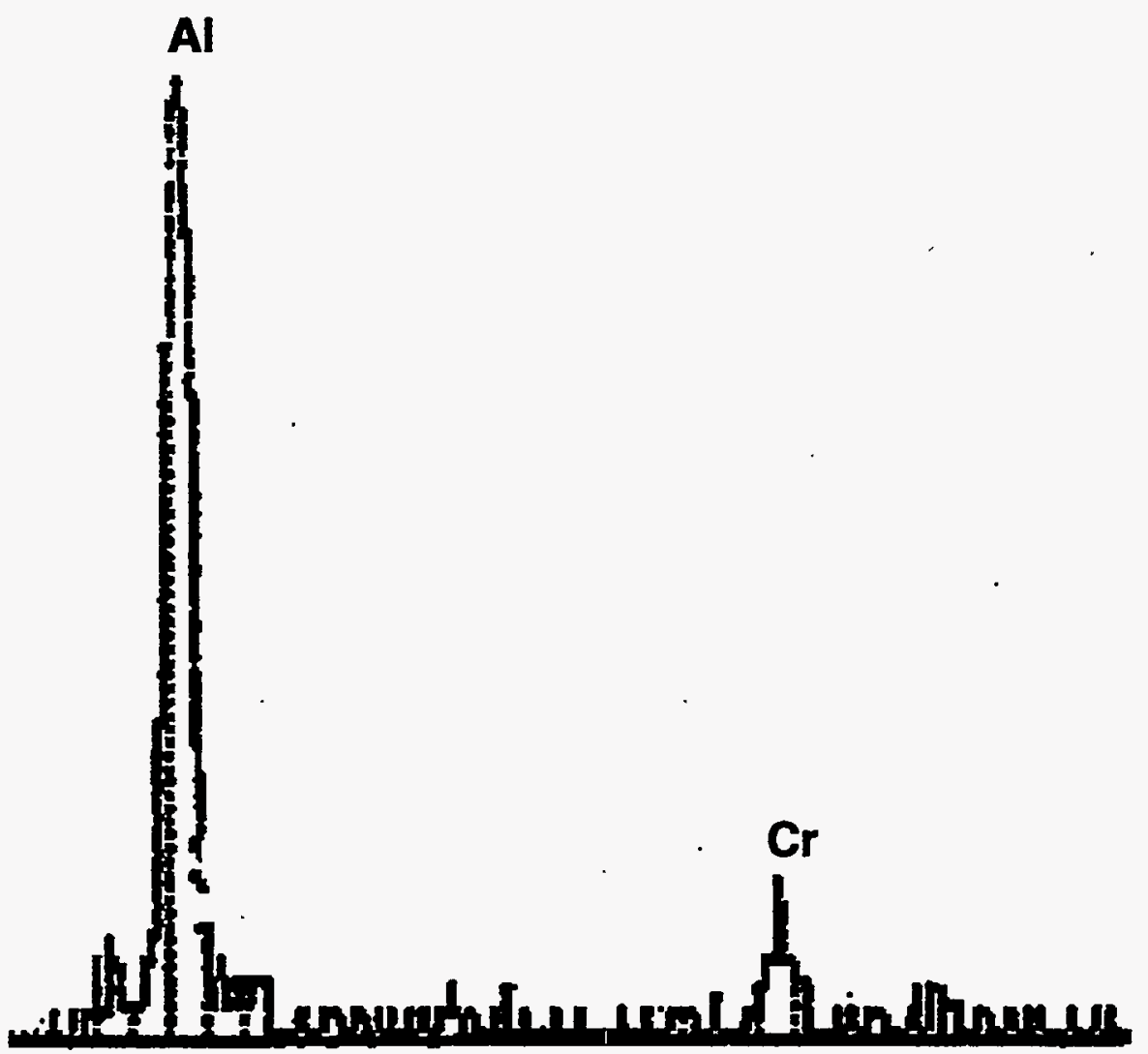

Figure A.14. EDS Spectrum of Al/CrParticle in Figure A.6 


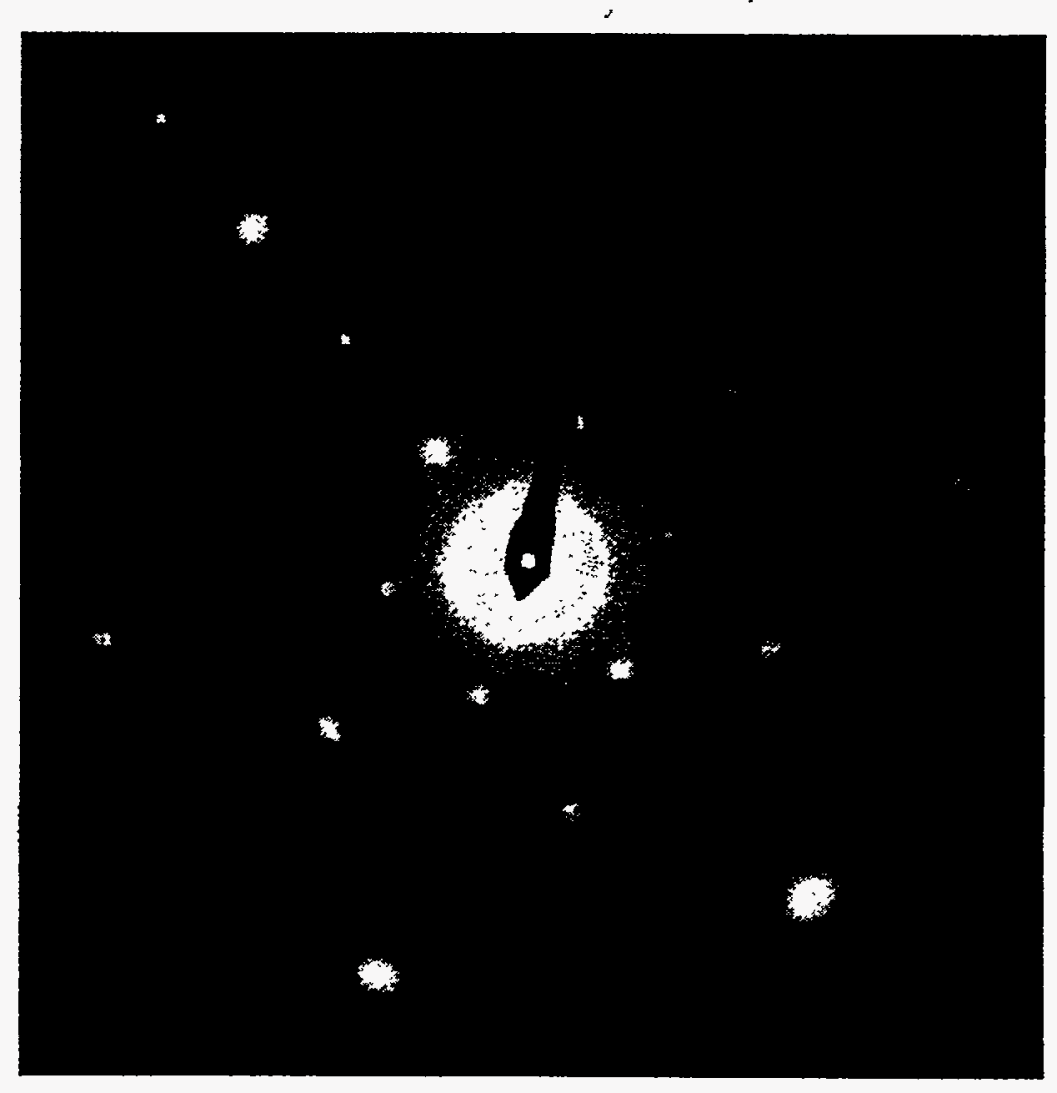

Figure A.15. Selected Area Diffraction (SAD) Pattern from g- $\mathrm{Al}_{2} \mathrm{O}_{3}$ Shown in Figure A.2 


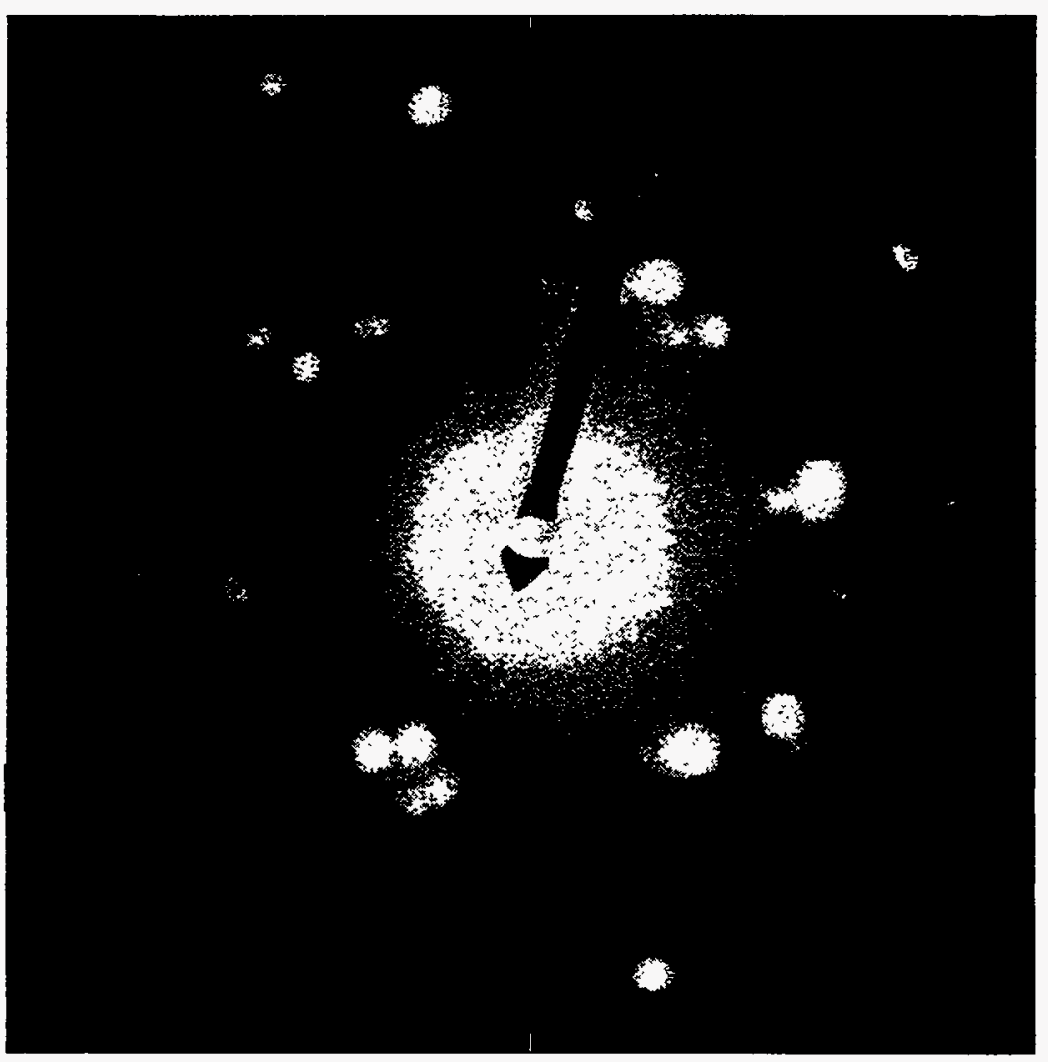

Figure A.16. SAD Pattern from FeOOH Particles Shown in Figure A.3 


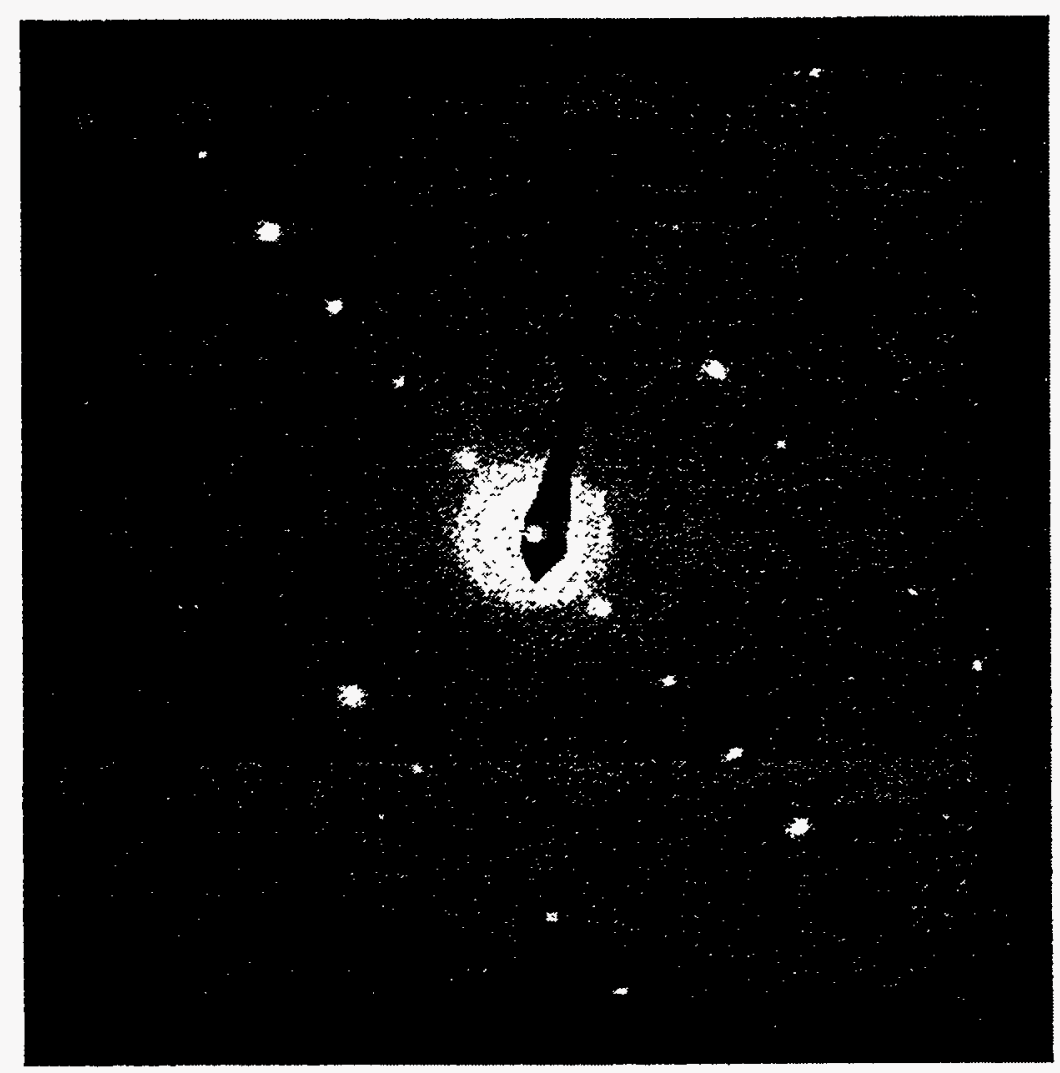

Figure A.17. $\mathrm{SAD}$ Pattern from $\mathrm{Al}_{2} \mathrm{Si}_{4} \mathrm{O}_{10}$ Particles Shown in Figure A.5 


\section{Appendix B}

\section{Particle Size Analysis Results}




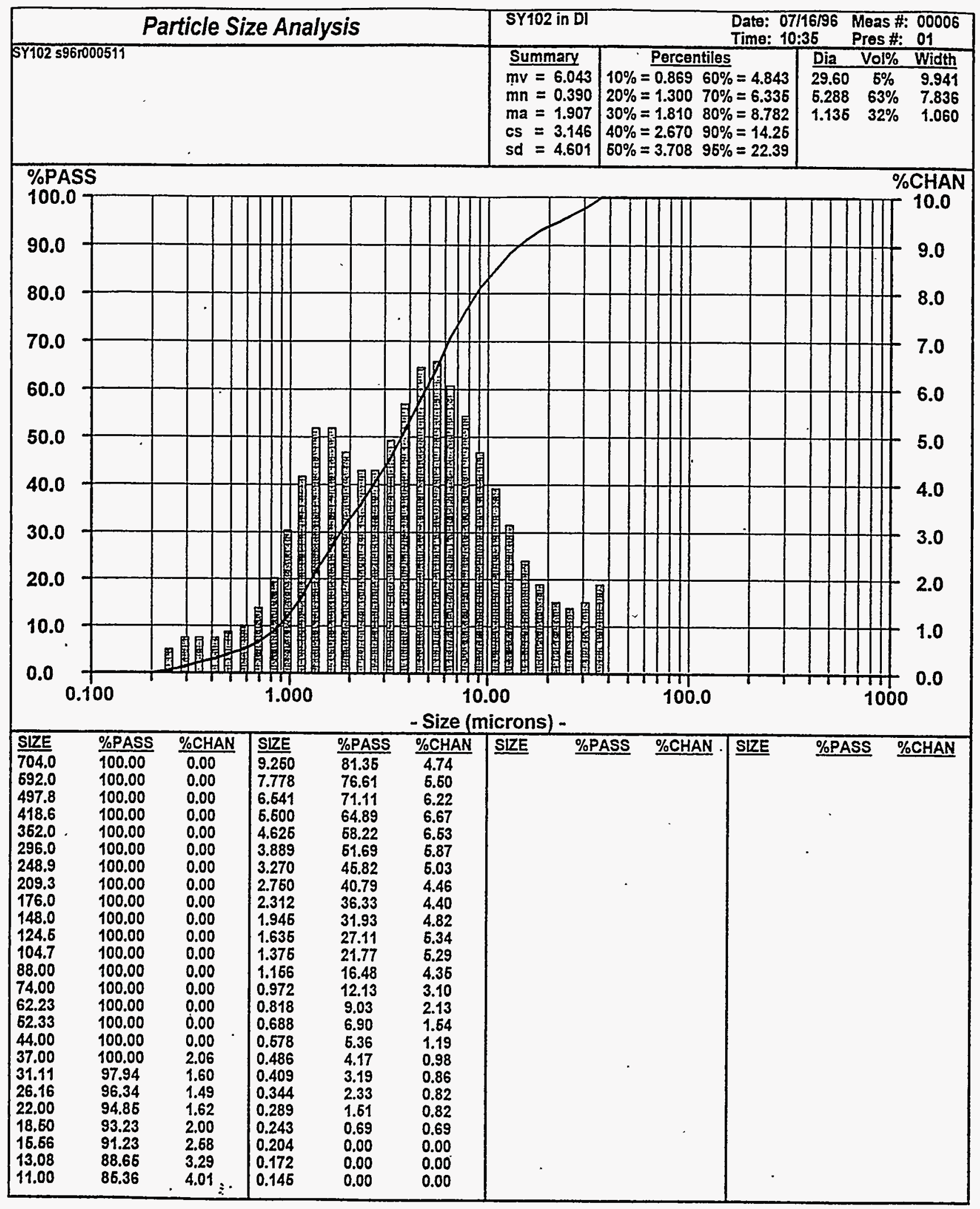

Figure B.1. Particle Size Distribution of SY-102 Sample in DI Water (s96r000511 Sample)

B.1 ${ }^{\prime}$ 


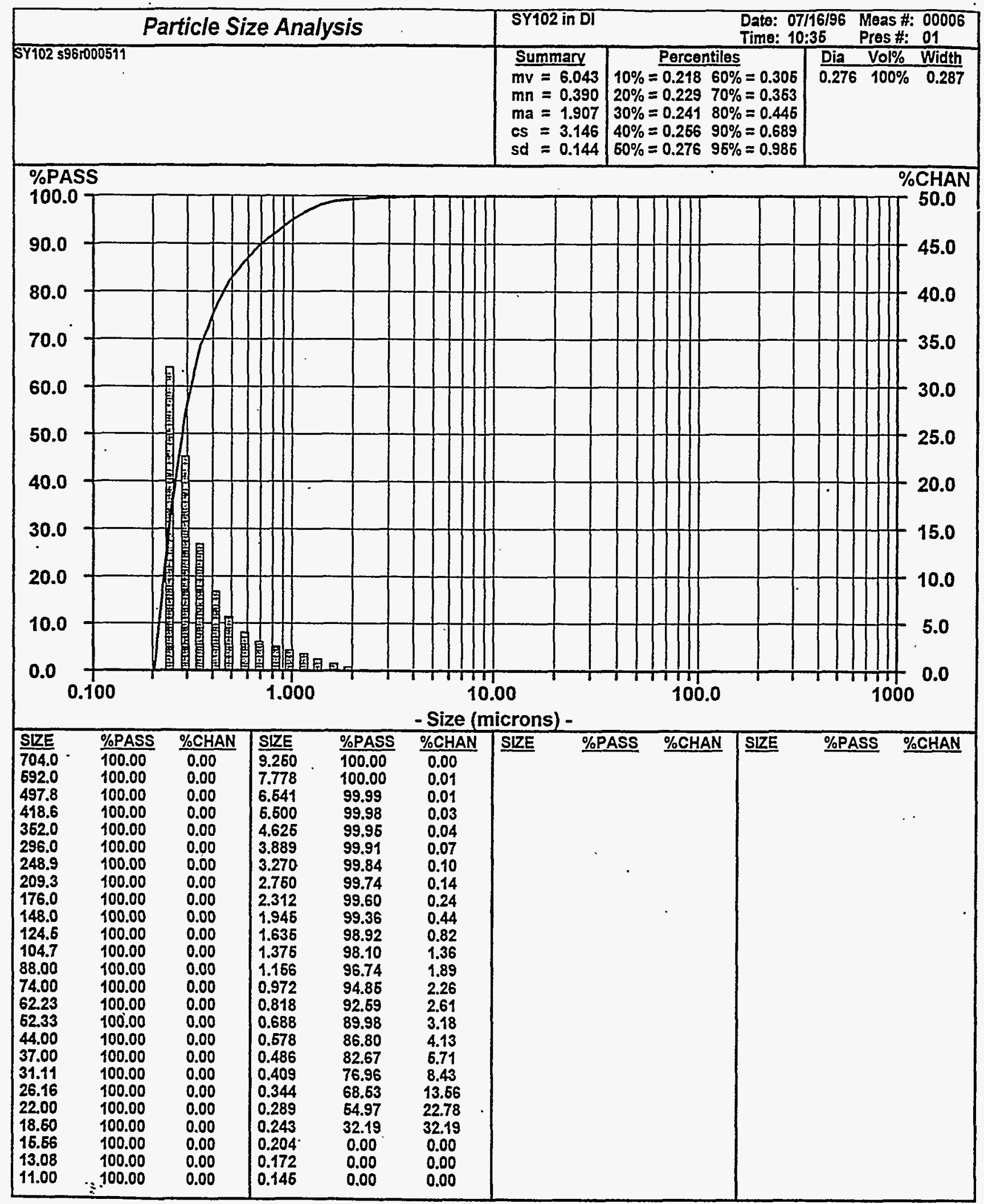

Figure B.2. Particle Size Distribution of SY-102 Sample in DI Water Sonicated for 300 seconds (s96r000511 Sample) 


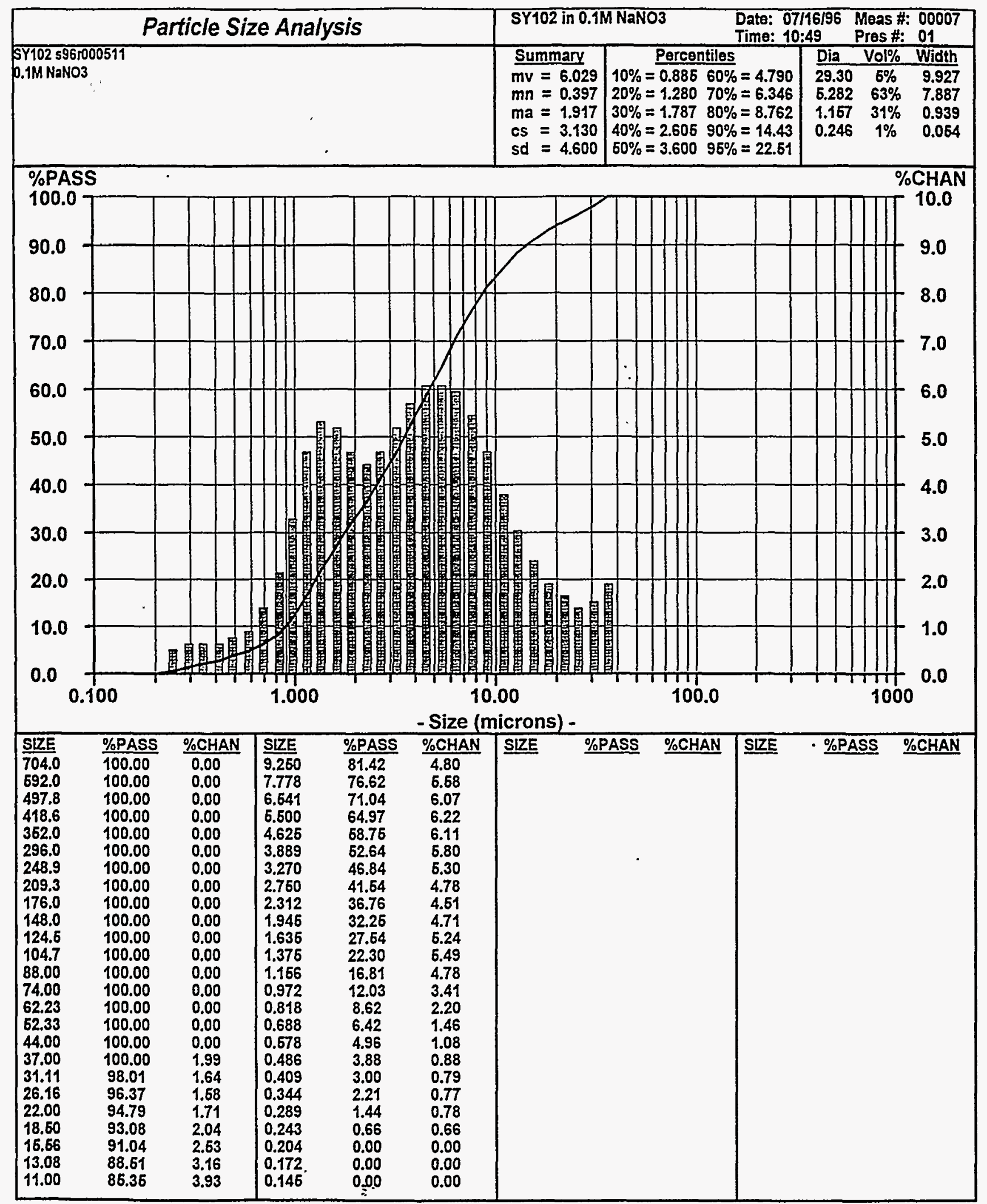

Figure B.3. Particle Size Distribution of SY-102 Sample in 0.1 M NaNO $\mathrm{Nal}_{3}$ Solution (s96r000511 Sample). 


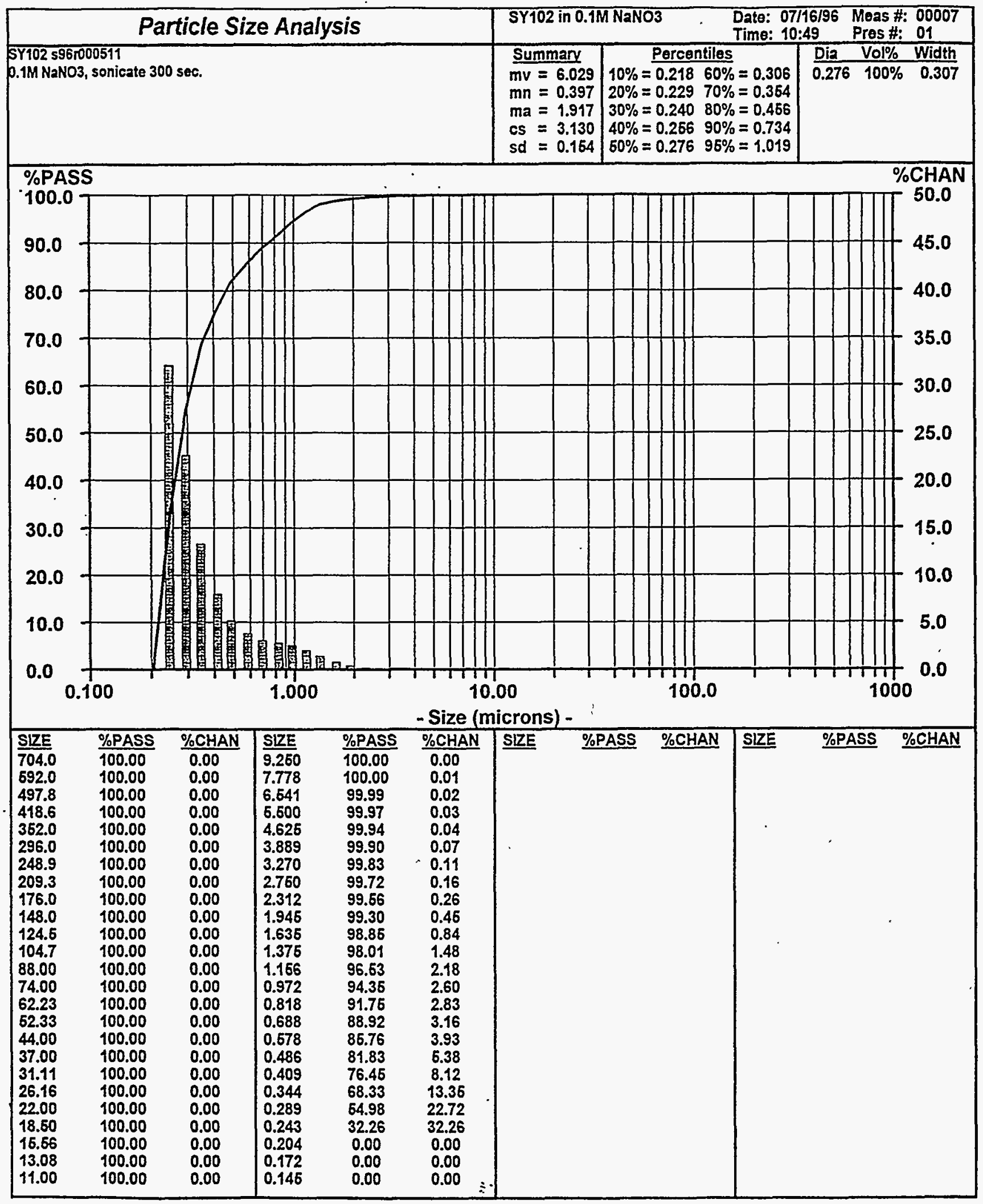

Figure B.4. Particle Size Distribution of SY-102 Sample in $0.1 \mathrm{M} \mathrm{NaNO}_{3}$ Solution Sonicated for 300 s (s96r000511 Sample) 


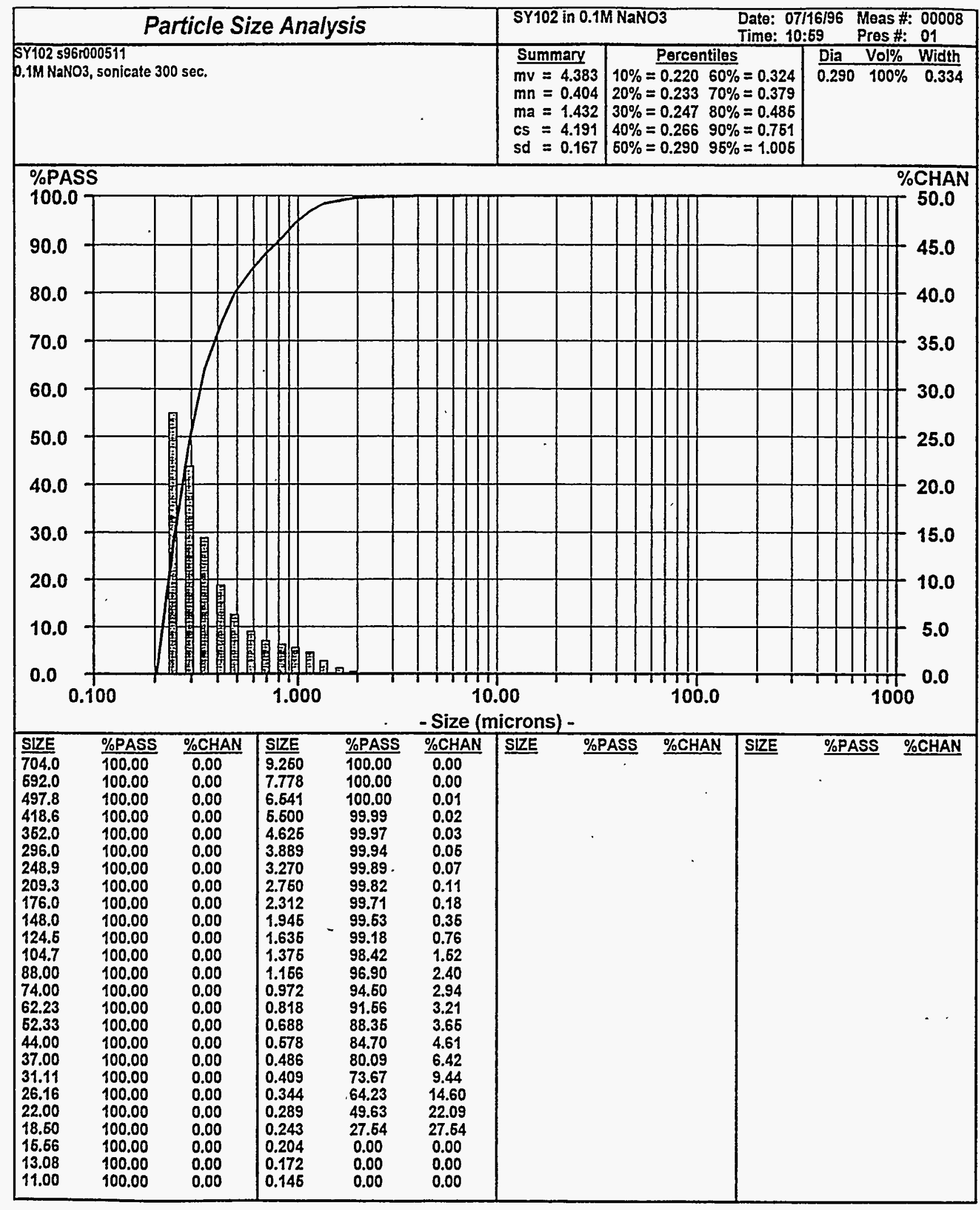

Figure B.5. Particle Size Distribution of SY-102 Sample in $0.1 \underline{\mathrm{M}} \mathrm{NaNO}_{3}$ Solution Sonicated for 300 s (s96r0.00511 Sample) - Rerun 


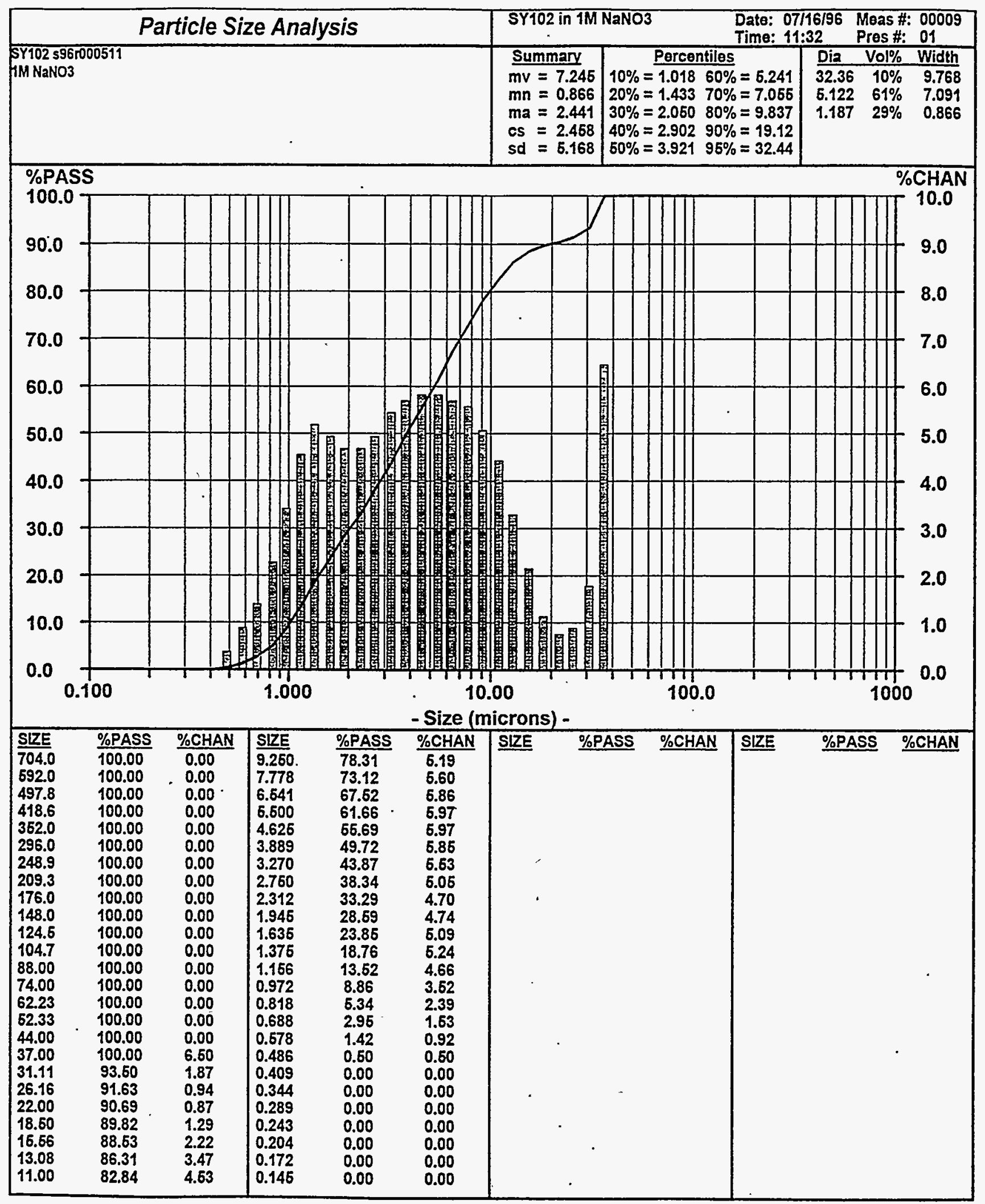

Figure B.6. Particle Size Distribution of SY-102 Sample in $1 \underline{\mathrm{M}} \mathrm{NaNO}_{3}$ Solution . (s96r000511 Sample) 


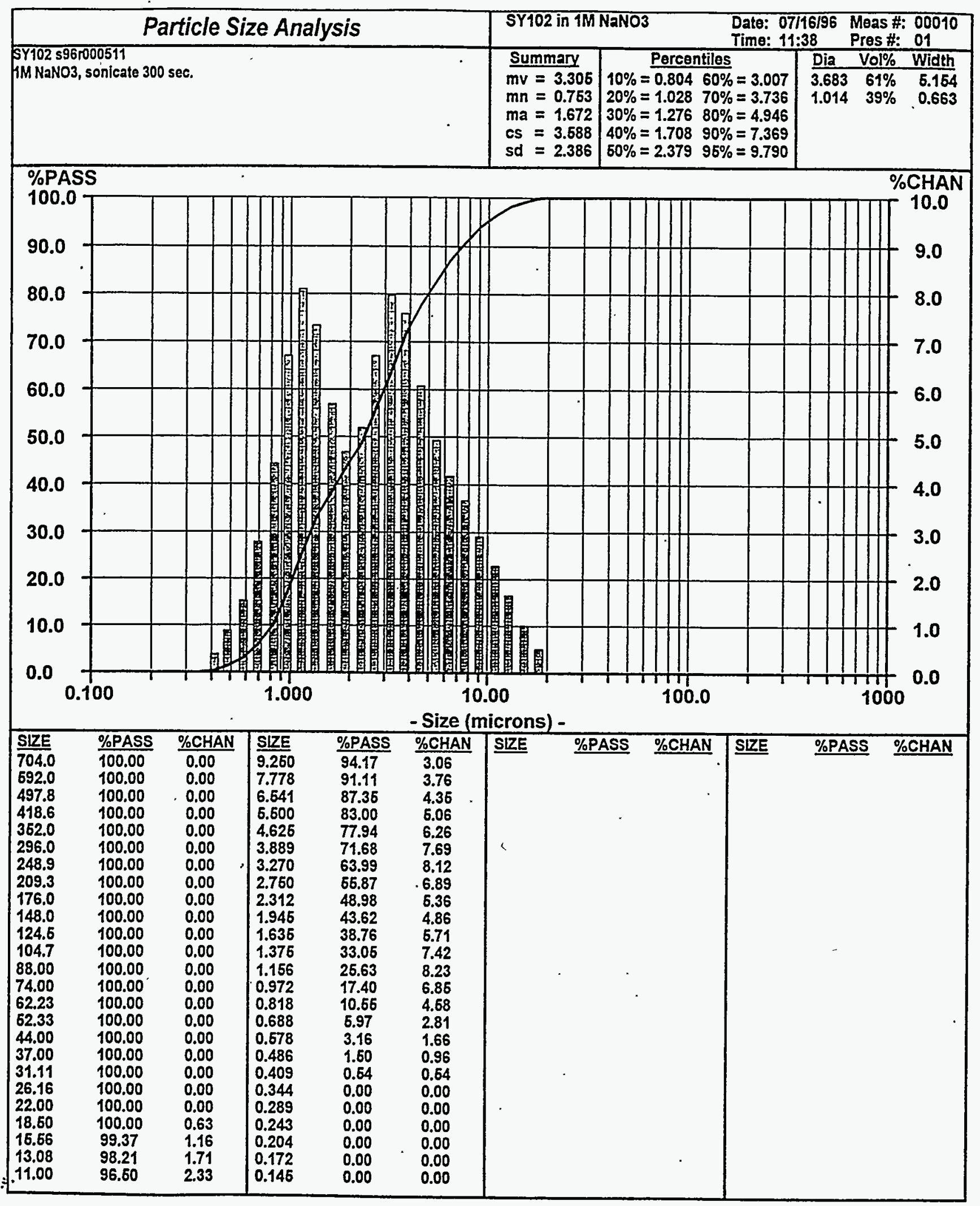

Figure B.7. Particle Size Distribution of SY-102 Sample in $1 \underline{\mathrm{M}} \mathrm{NaNO}_{3}$ Solution Sonicated for $300 \mathrm{~s}$ (s96r000511 Sample) 


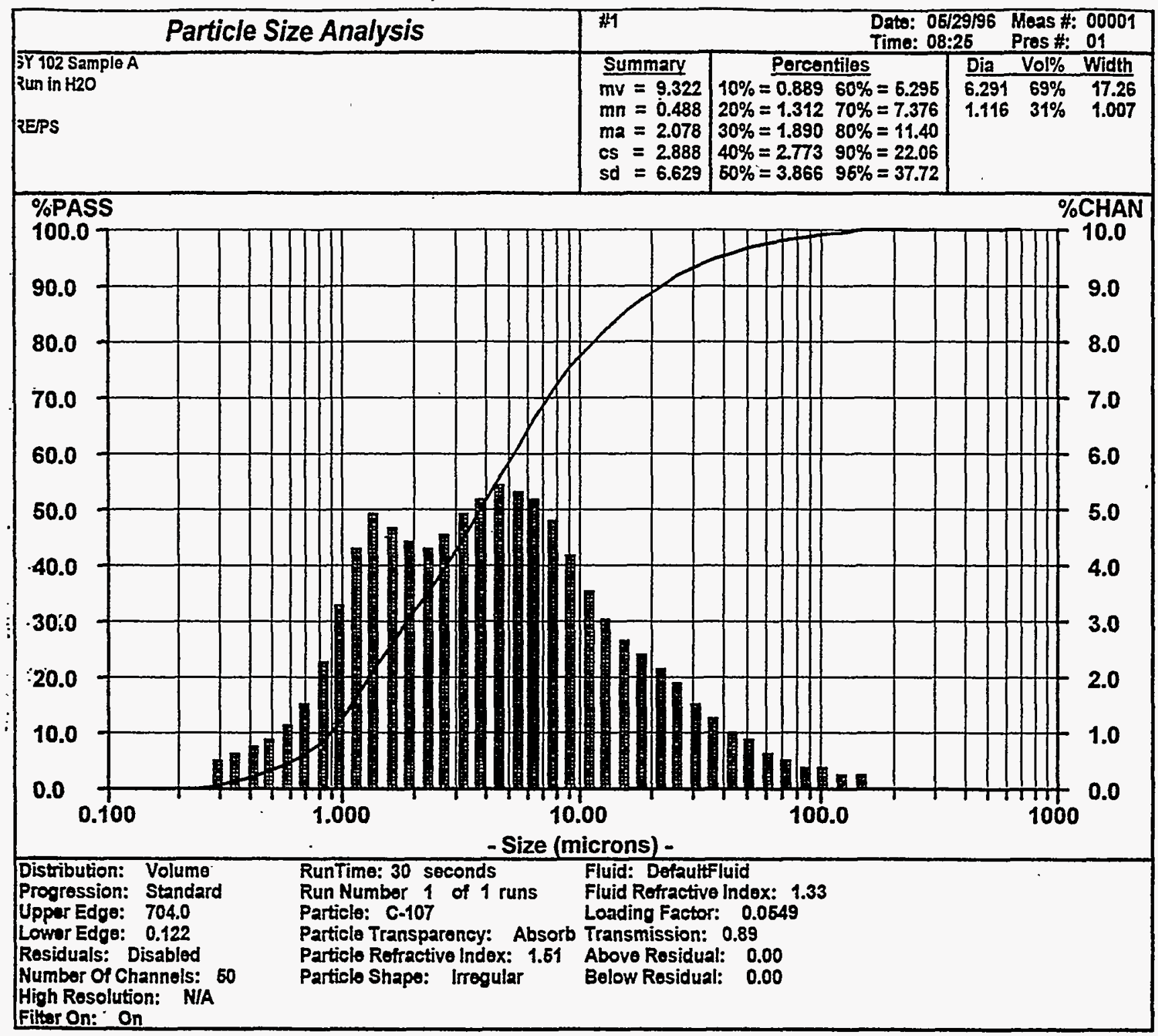

Figure B.8. Particle Size Distribution of SY-102 Sample in DI Water (Vial A Sample-Run \#1) 


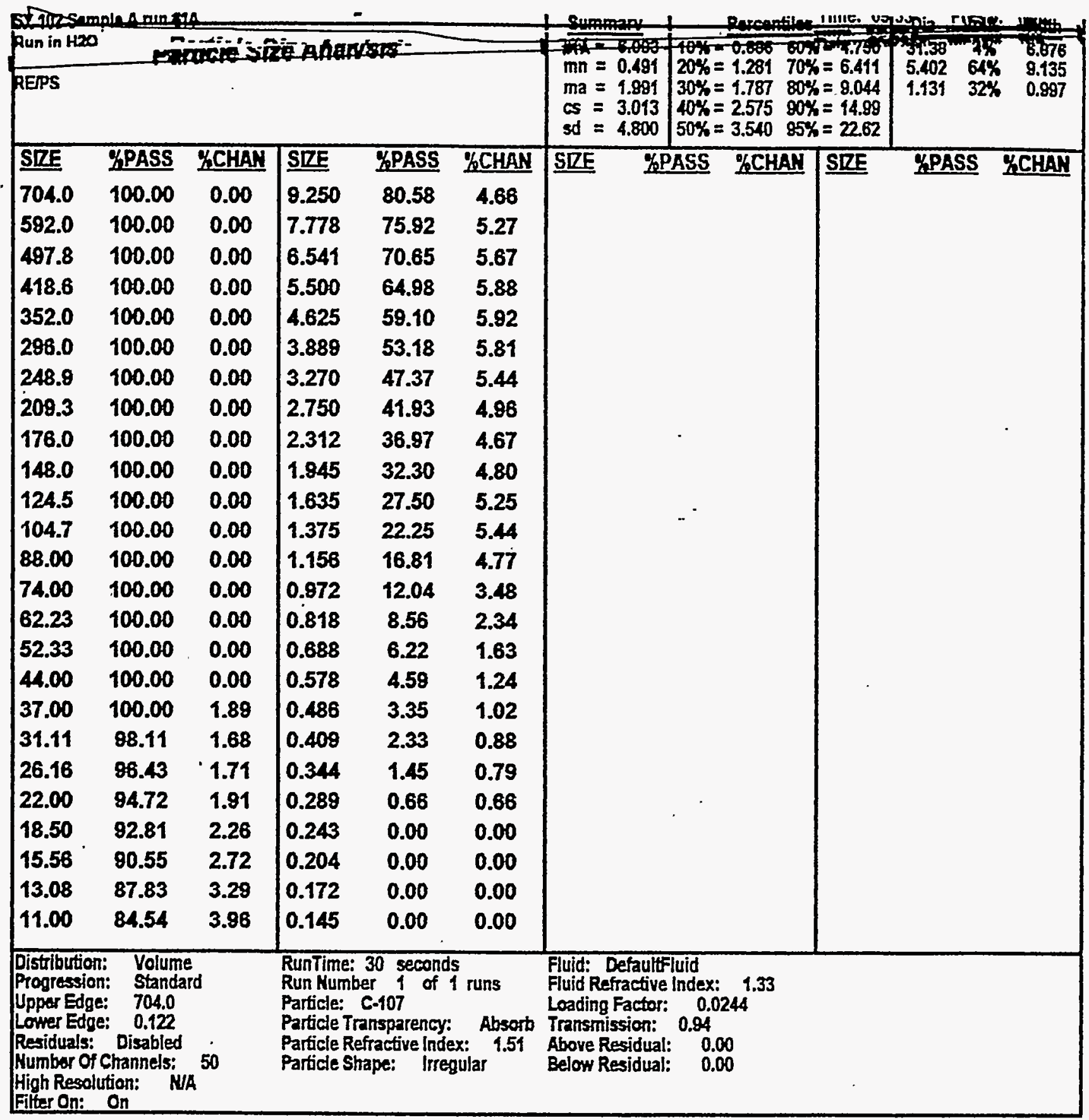

Figure B.8 (contd). Particle Size Distribution of SY-102 Sample in DI Water (Vial A Sample - Run \#1) 


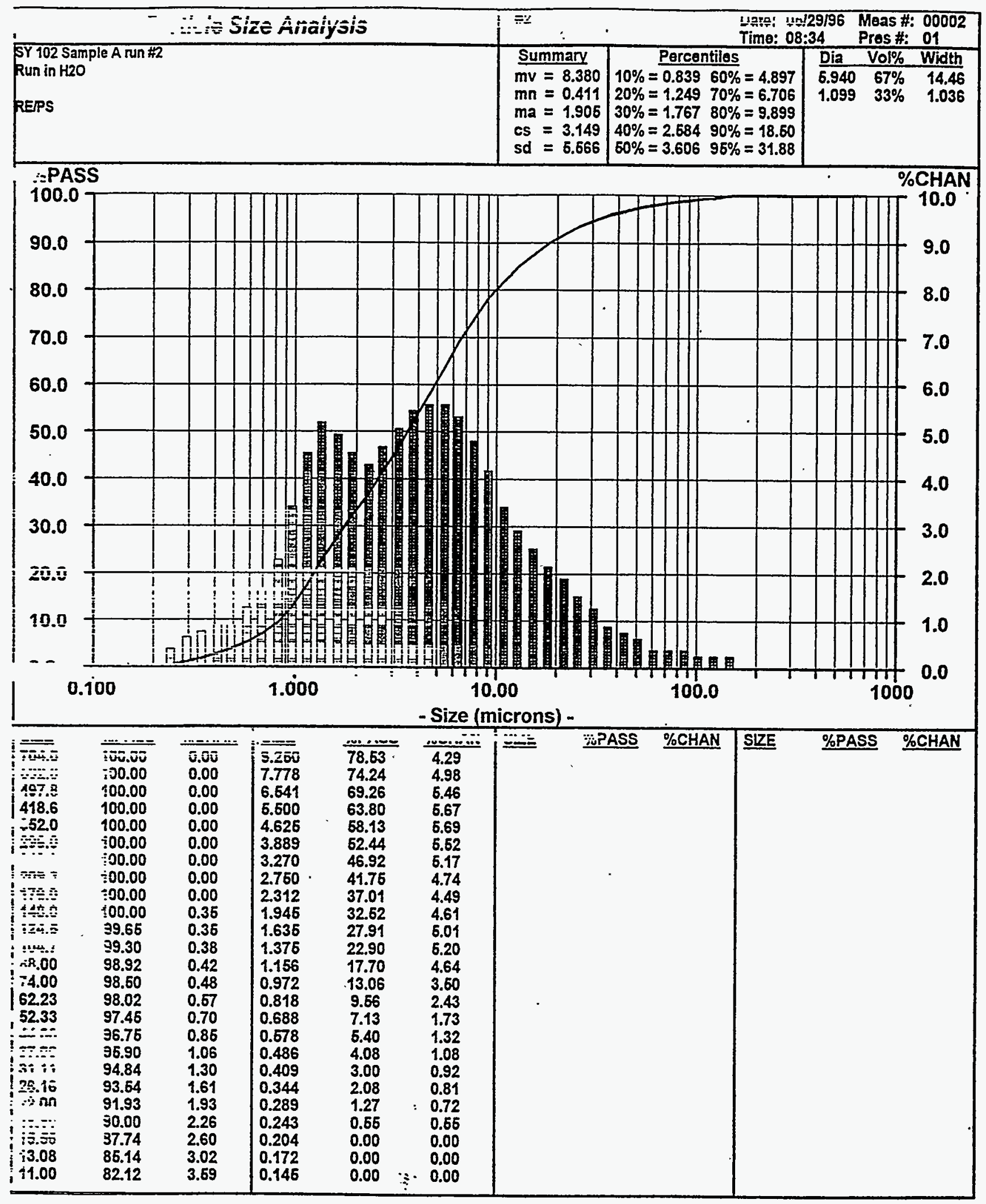

Figure B.9. Particle Size Distribution of SY-102 Sample in DI Water (Vial A Sample - Run\#2) 


\begin{tabular}{|c|c|c|c|c|c|c|c|c|c|c|}
\hline \multicolumn{6}{|c|}{ Particle Size Analysis } & \multicolumn{5}{|c|}{$\begin{array}{l}\text { Date: 06/29/96 Meas \#: } 00002 \\
\text { Time: 08:34 Pros \#: } 01\end{array}$} \\
\hline \multicolumn{6}{|c|}{$\begin{array}{l}\text { SY } 102 \text { Sample A nun H2 } \\
\text { Run in } \mathrm{H} 20 \\
\text { REPS }\end{array}$} & $\begin{array}{l}\text { Summany } \\
\mathrm{mv}=8.380 \\
\mathrm{mn}=0.411 \\
\mathrm{ma}=1.905 \\
\mathrm{cs}=3.149 \\
\mathrm{sd}=6.666\end{array}$ & \multicolumn{2}{|c|}{$\begin{array}{l}\text { Porcentiles } \\
10 \%=0.83960 \%=4.897 \\
20 \%=1.249 \quad 70 \%=6.706 \\
30 \%=1.767 \quad 80 \%=9.899 \\
40 \%=2.68490 \%=18.60 \\
60 \%=3.606 \quad 96 \%=31.88\end{array}$} & \multirow{2}{*}{\multicolumn{2}{|c|}{$\begin{array}{ccc}\text { Die } & \text { Vol\% } & \text { Width } \\
5.940 & 67 \% & 14.46 \\
1.099 & 33 \% & 1.036 \\
& & \\
& & \\
\text { \%PASS } & \text { \%CHAN } \\
\end{array}$}} \\
\hline SIZE & \%PASS & $\%$ CHAN & SIZE & \%PASS & \%CHAN & \multirow[t]{26}{*}{ SIZE $\quad \%$ PAS } & \multirow[t]{26}{*}{ S \%CHAN } & \multirow[t]{26}{*}{ SIZE } & & \\
\hline 704.0 & 100.00 & 0.00 & 9.250 & 78.53 & 4.29 & & & & \multirow{25}{*}{$r$} & \\
\hline 592.0 & 100.00 & 0.00 & 7.778 & 74.24 & 4.98 & & & & & \\
\hline 497.8 & 100.00 & 0.00 & 6.541 & 69.26 & 5.46 & & & & & \\
\hline 418.6 & 100.00 & 0.00 & 5.500 & 63.80 & 5.67 & & & & & \\
\hline 352.0 & 100.00 & 0.00 & 4.625 & 58.13 & 5.69 & & & & & \\
\hline 296.0 & 100.00 & 0.00 & 3.889 & 52.44 & 5.52 & & & & & \\
\hline 248.9 & 100.00 & 0.00 & 3.270 & 46.92 & 5.17 & & & & & \\
\hline 209.3 & 100.00 & 0.00 & 2.750 & 41.75 & 4.74 & & & & & \\
\hline 176.0 & 100.00 & 0.00 & 2.312 & 37.01 & 4.49 & & & & & \\
\hline 148.0 & 100.00 & 0.35 & 1.945 & 32.52 & 4.61 & & & & & \\
\hline 124.5 & 99.65 & 0.35 & 1.635 & 27.91 & 5.01 & & & & & \\
\hline 104.7 & 99.30 & 0.38 & 1.375 & 22.90 & 5.20 & & & & & \\
\hline 88.00 & 98.92 & 0.42 & 1.156 & 17.70 & 4.64 & & & & & \\
\hline 74.00 & 98.50 & 0.48 & 0.972 & 13.06 & 3.50 & & & & & \\
\hline 62.23 & 98.02 & 0.57 & 0.818 & 9.56 & 2.43 & & & & & \\
\hline 52.33 & 97.45 & 0.70 & 0.688 & 7.13 & 1.73 & & & & & \\
\hline 44.00 & 96.75 & 0.85 & 0.578 & 5.40 & 1.32 & & & & & \\
\hline 37.00 & 95.90 & 1.06 & 0.486 & 4.08 & 1.08 & & & & & \\
\hline 31.11 & 94.84 & 1.30 & 0.409 & 3.00 & 0.92 & & & & & \\
\hline 26.16 & 93.54 & 1.61 & 0.344 & 2.08 & 0.81 & & & & & \\
\hline 22.00 & 91.93 & 1.93 & 0.289 & 1.27 & 0.72 & & & & & \\
\hline 18.50 & 90.00 & 2.26 & 0.243 & 0.55 & 0.55 & & & & & \\
\hline 15.56 & 87.74 & 2.60 & 0.204 & 0.00 & 0.00 & & & & & \\
\hline 13.08 & 85.14 & 3.02 & 0.172 & 0.00 & 0.00 & & & & & \\
\hline 11.00 & 82.12 & 3.59 & 0.145 & 0.00 & 0.00 & & & & & \\
\hline \multicolumn{3}{|c|}{$\begin{array}{l}\text { Distribution: Volume } \\
\text { Progression: Standard } \\
\text { Upper Edge: } 704.0 \\
\text { Lower Edge: 0.122 } \\
\text { Residuals: Disabled } \\
\text { Number Of Channels: } 60 \\
\text { High Rosolution: NIA } \\
\text { Filter On: On } \\
\end{array}$} & \multicolumn{3}{|c|}{$\begin{array}{l}\text { RunTime: } 30 \text { seconds } \\
\text { Run Number } 1 \text { of } 1 \text { runs } \\
\text { Particle: C-107 } \\
\text { Particle Transparency: Absorb } \\
\text { Particle Refractive Index: } 1.61 \\
\text { Particle Shapo: Irrogular }\end{array}$} & \multicolumn{3}{|c|}{$\begin{array}{l}\text { Fluid: DofaulfFluid } \\
\text { Fluid Refractive Index: } 1.33 \\
\text { Loading Factor: } 0.0620 \\
\text { Transmission: } 0.90 \\
\text { Above Residual: } 0.00 \\
\text { Bolow.Residual: } 0.00\end{array}$} & & \\
\hline
\end{tabular}

Figure B.9 (Contd). Particle Size Distribution of SY-102 Sample in DI Water (Vial A Sample Run \#2) 


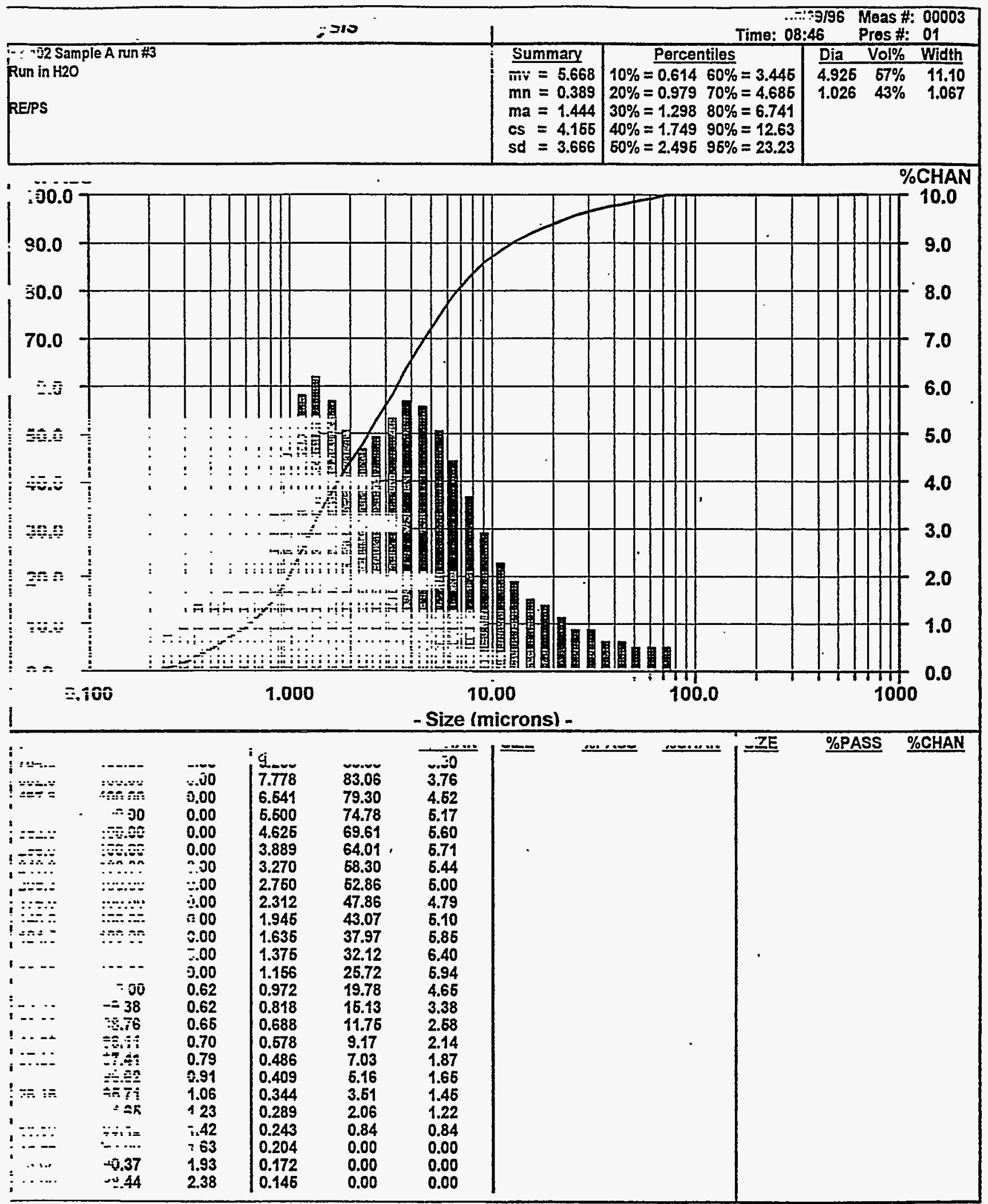

Figure B.10. Particle Size Distribution of SY-102 Sample in DI Water (Vial A Sample - Run \#3) 


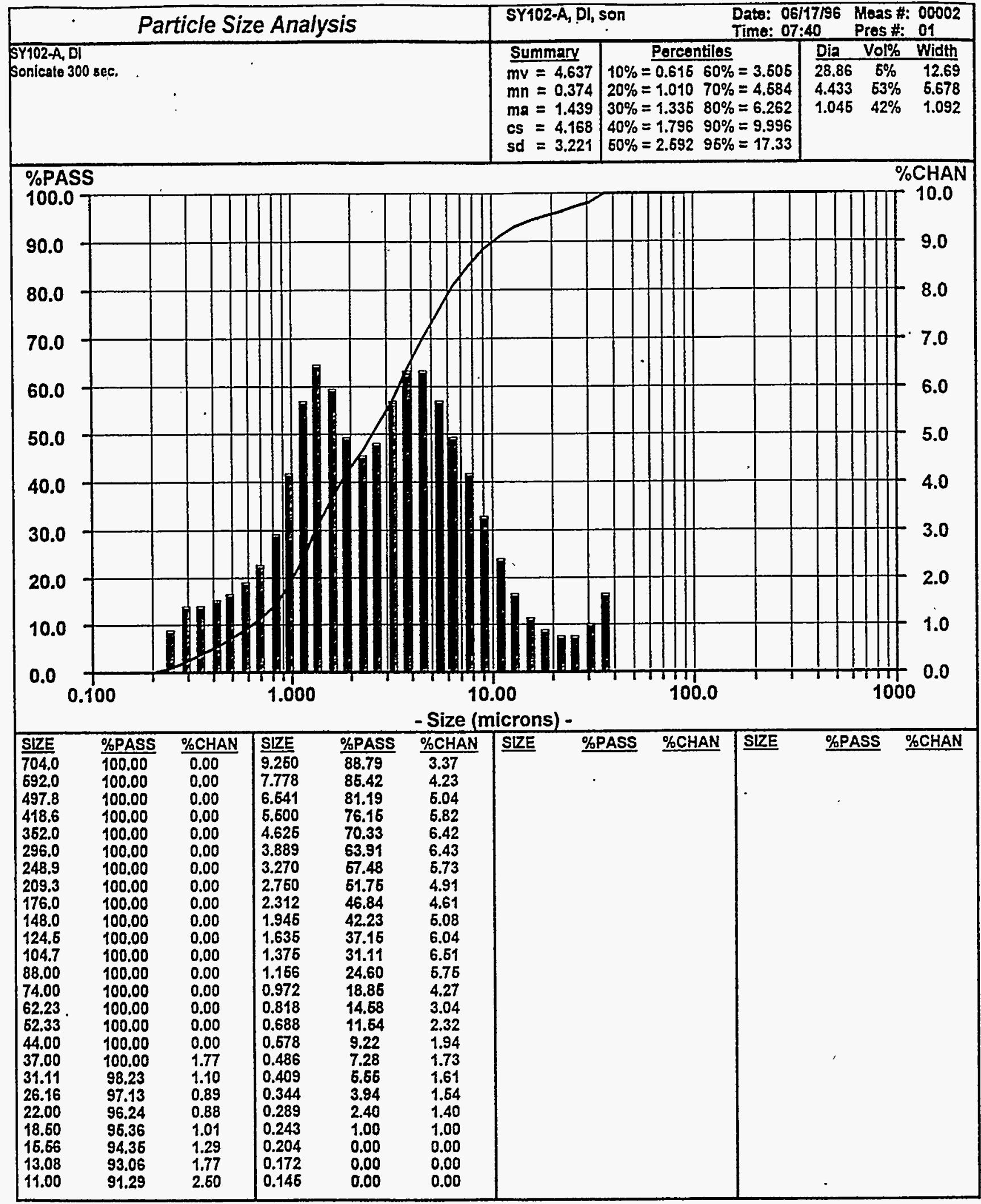

Figure B.11. Particle Size Distribution of SY-102 Sample in DI Water (Vial A Sample - Sonicated for $300 \mathrm{~s}$ ) 


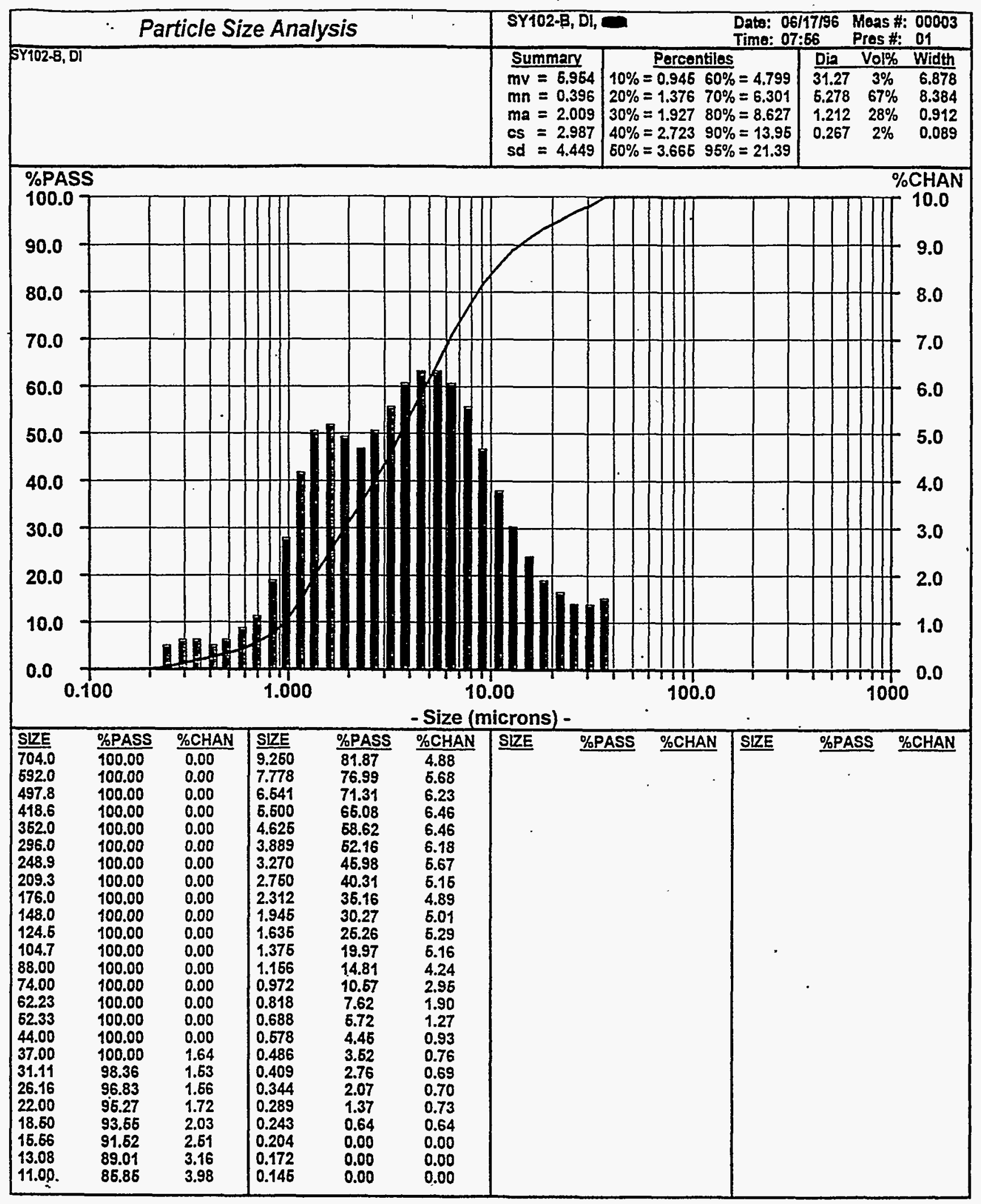

Figure B.12. Particle Size Distribution of SY-102 Sample in DI Water (Vial B Sample) 


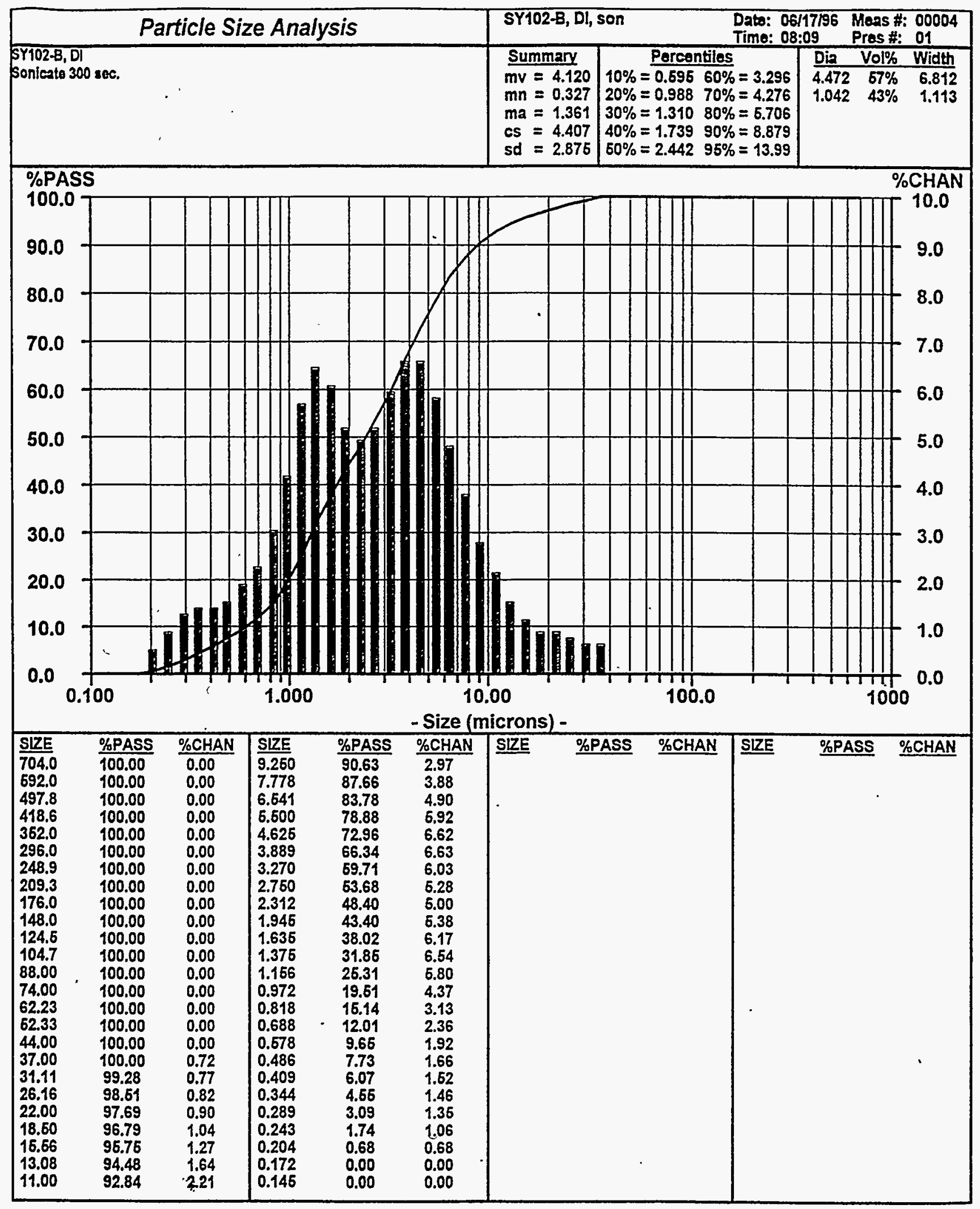

Figure B.13. Particle Size Distribution of SY-102 Sample in DI Water (Vial B Sample - Sonicated for $300 \mathrm{~s}$ ) 


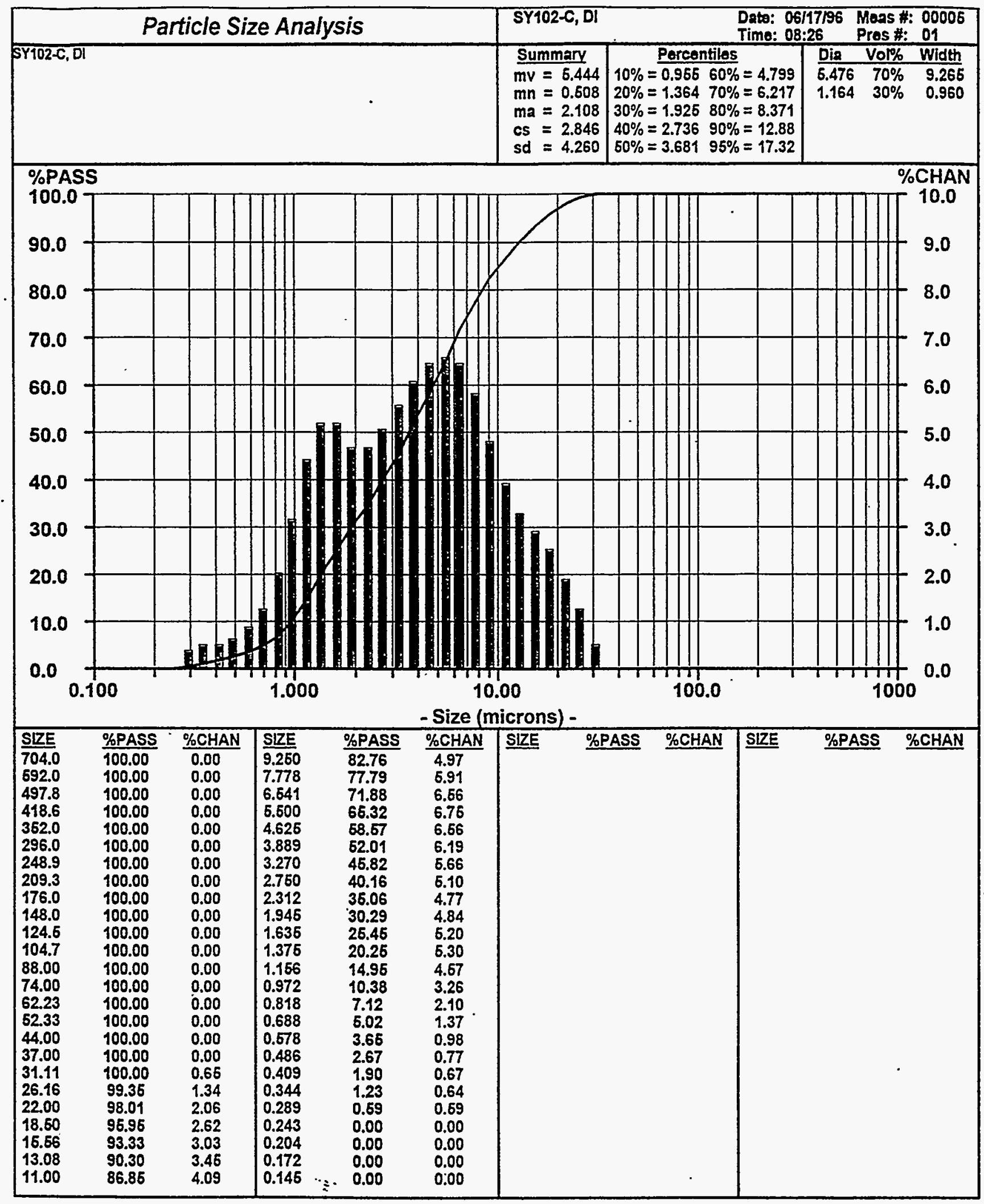

Figure B.14. Particle Size Distribution of SY-102 Sample in DI Water (Vial C Sample) 


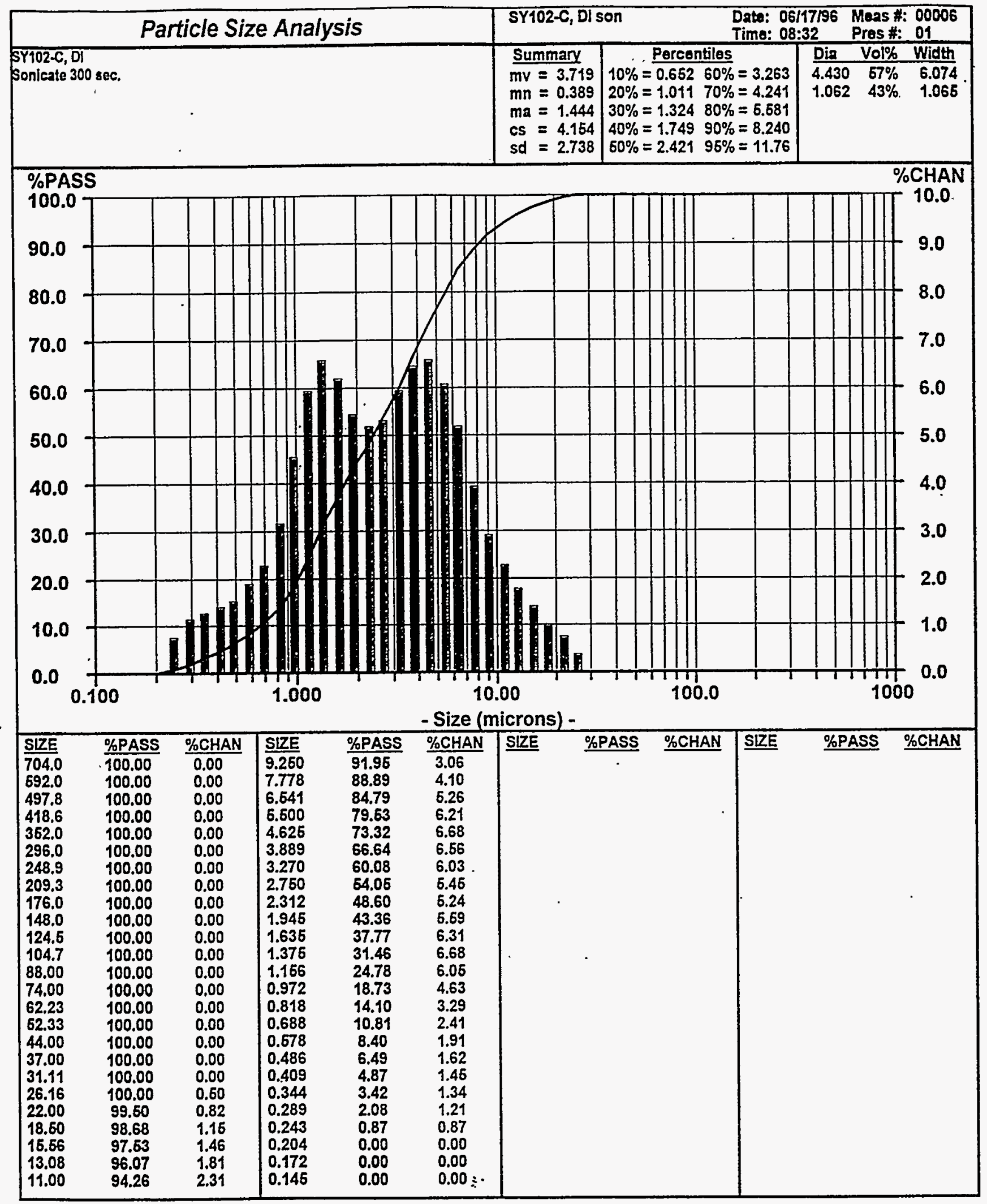

Figure B.15. Particle Size Distribution of SY-102 Sample in DI Water (Vial C Sample - Sonicated for $300 \mathrm{~s}$ ) 


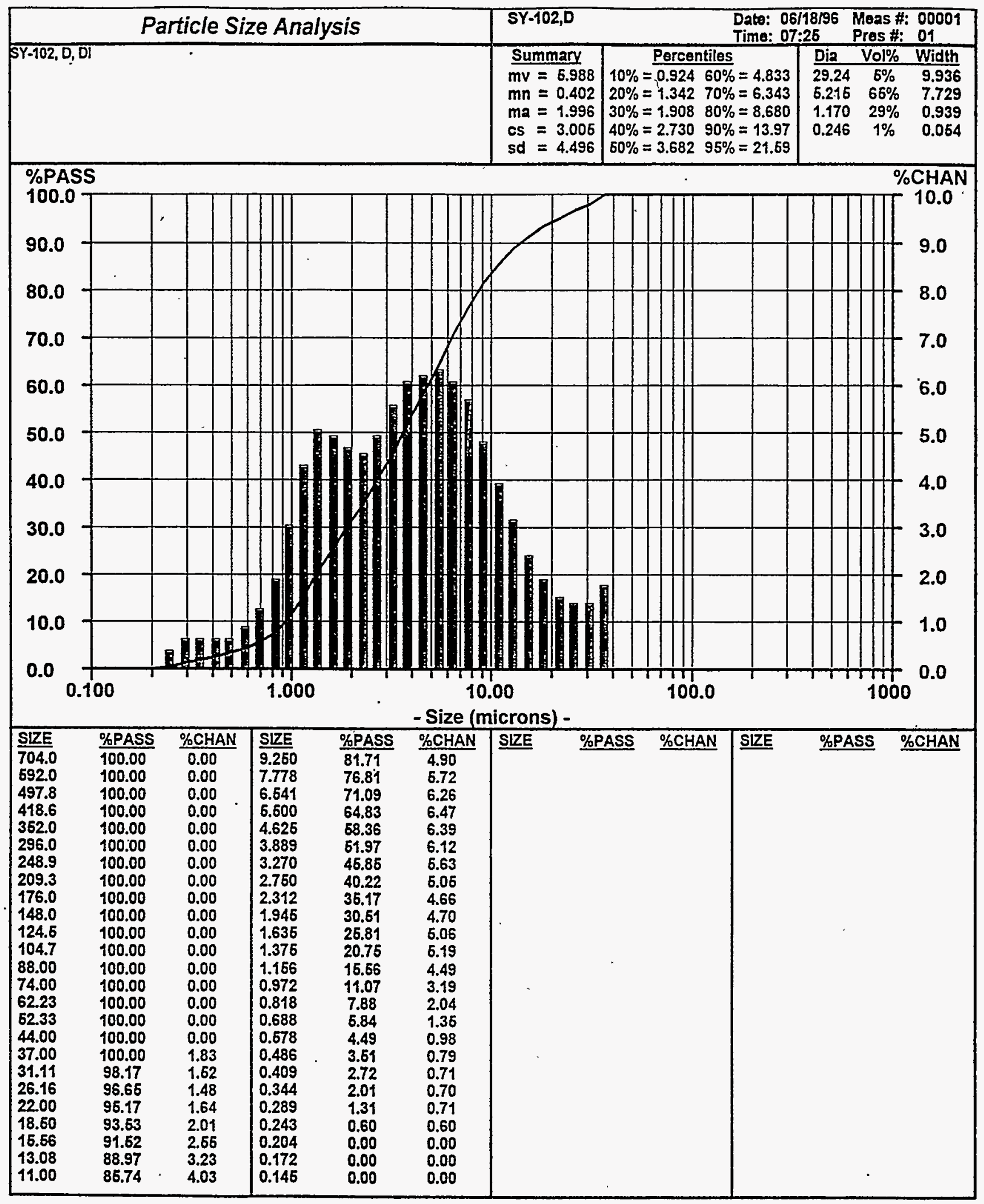

Figure B.16. Particle Size Distribution of SY-102 Sample in DI Water (Vial D Sample) 


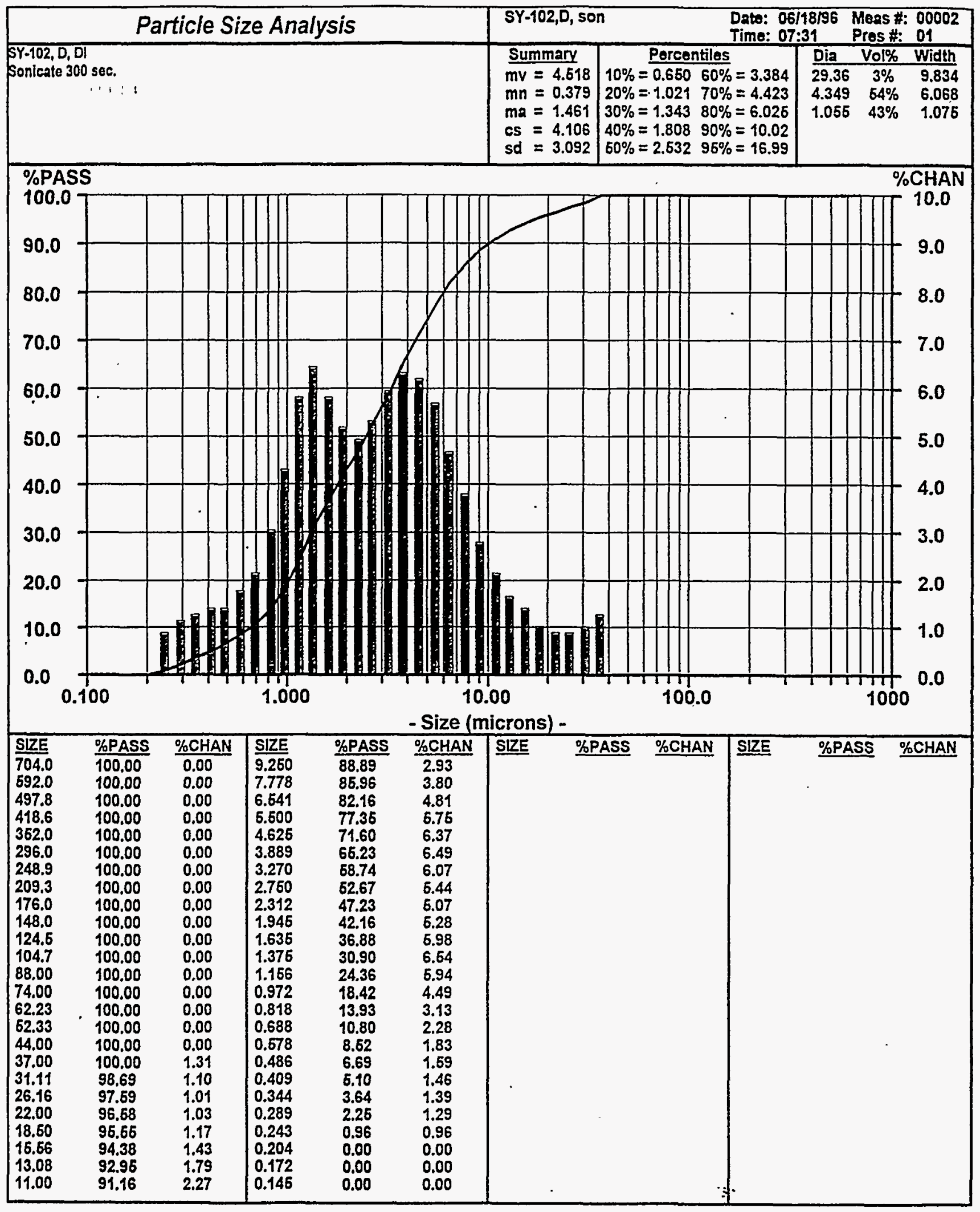

Figure B.17. Particle Size Distribution of SY-102 Sample in DI Water (Vial-D Sample - Sonicated for $300 \mathrm{~s}$ ) 


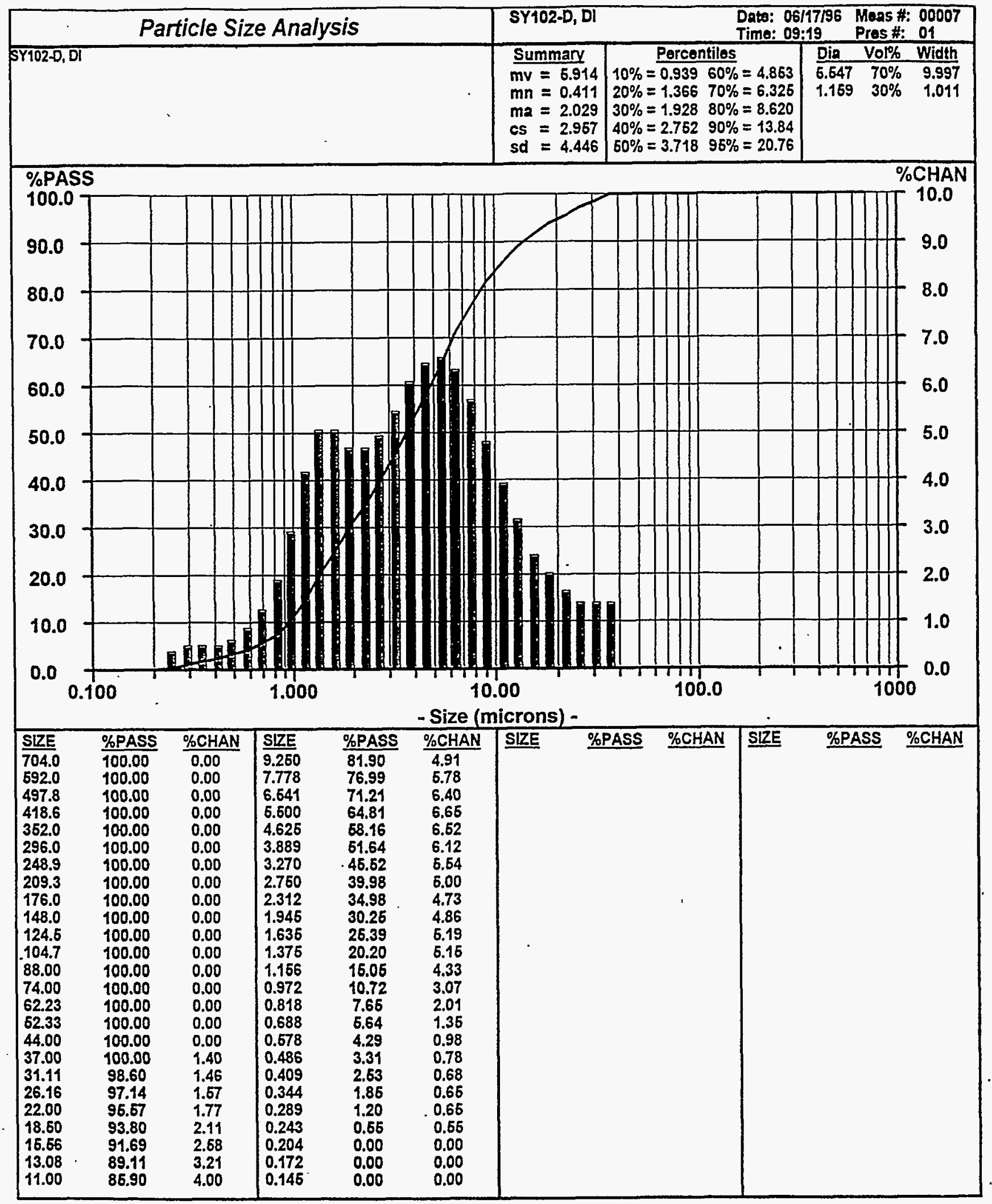

Figure B.18. Particle Size Distribution of SY-102 Sample in DI Water (Vial D Sample - Different Aliquot) 


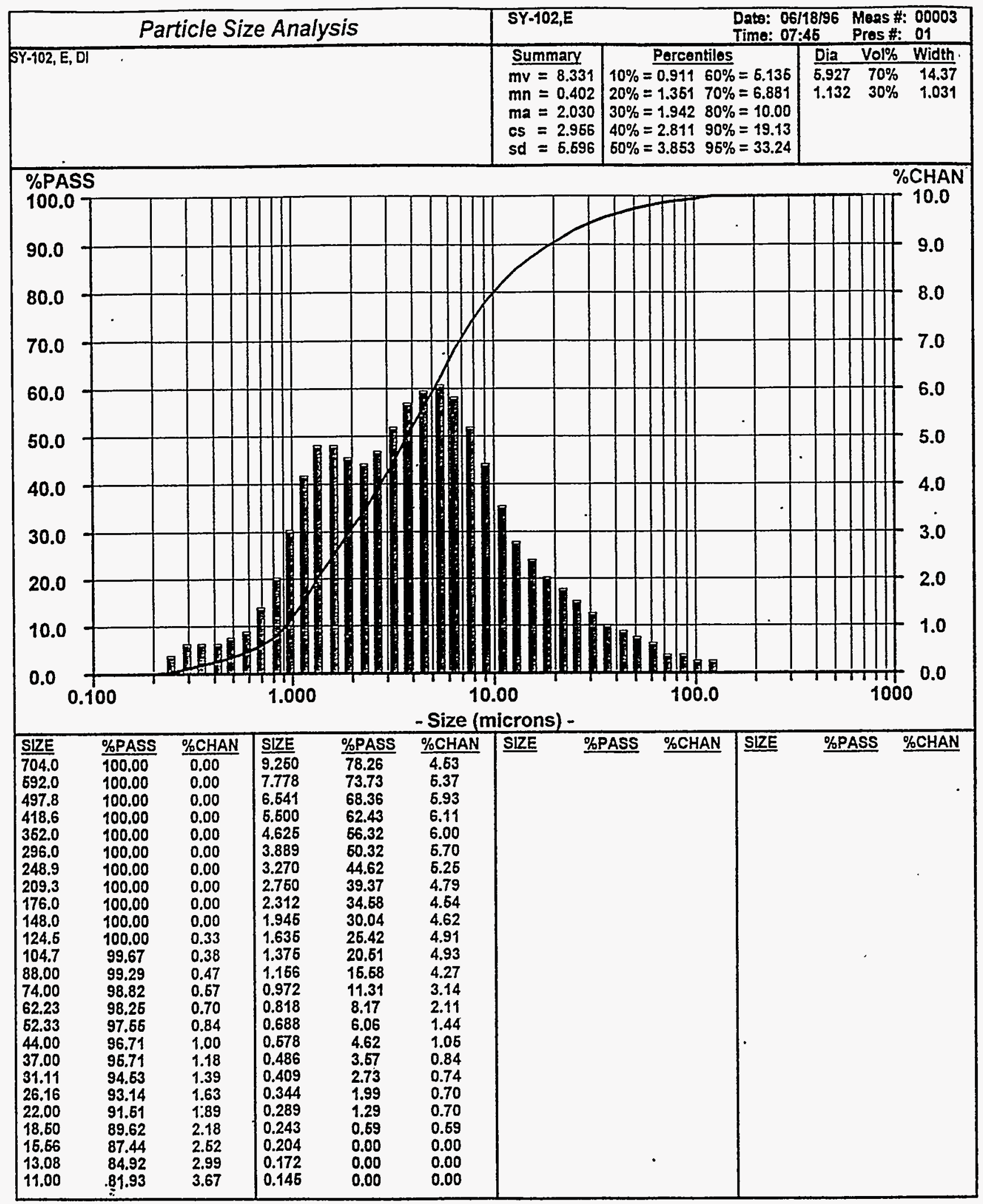

Figure B.19. Particle Size Distribution of SY-102 Sample in DI Water (Vial E Sample) 


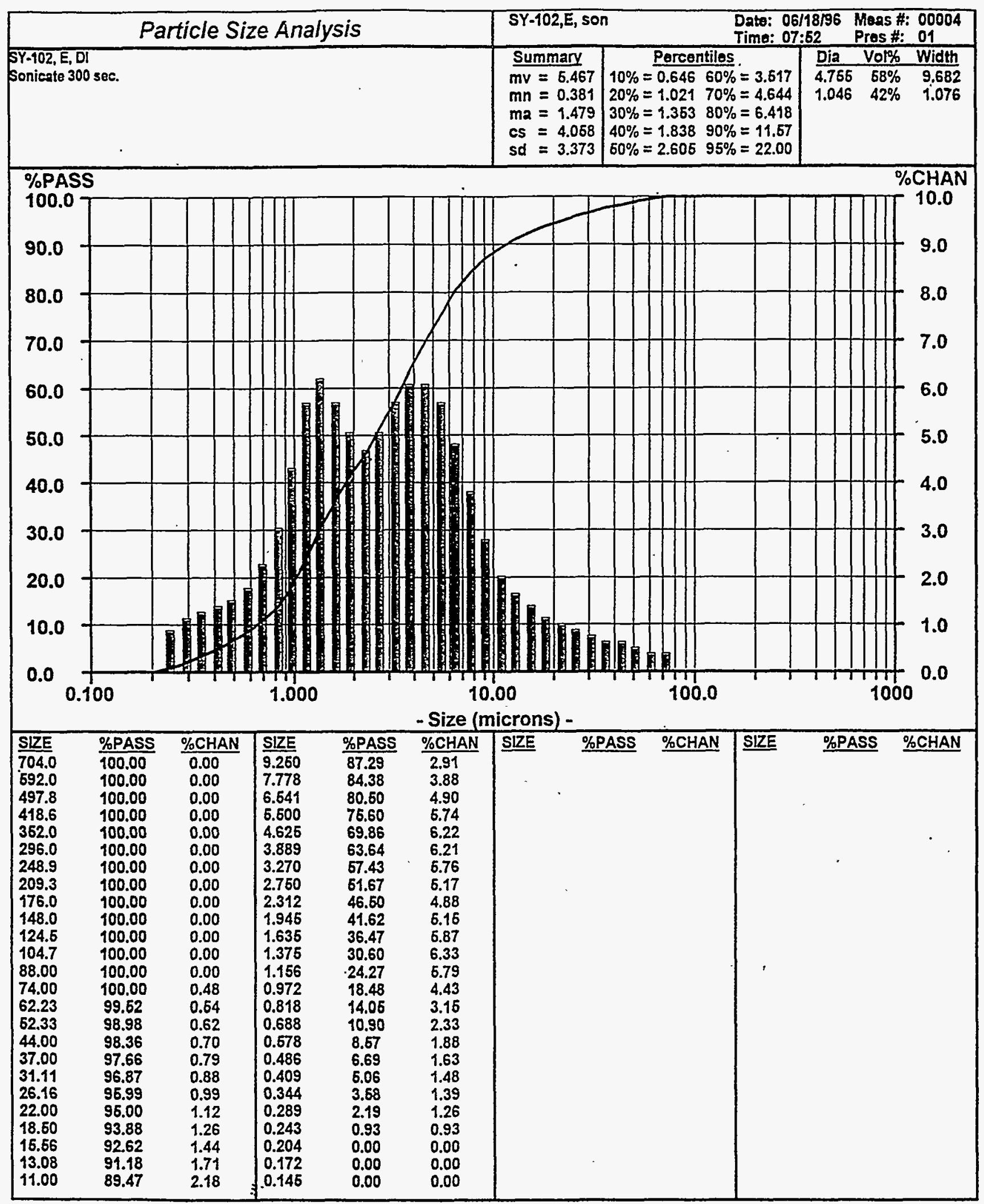

Figure B.20. Particle Size Distribution of SY-102 Sample in DI Water (Vial E Sample Sonicated for $300 \mathrm{~s}$ ) 
Appendix C

Pump Jet Mixing Simulation Results 



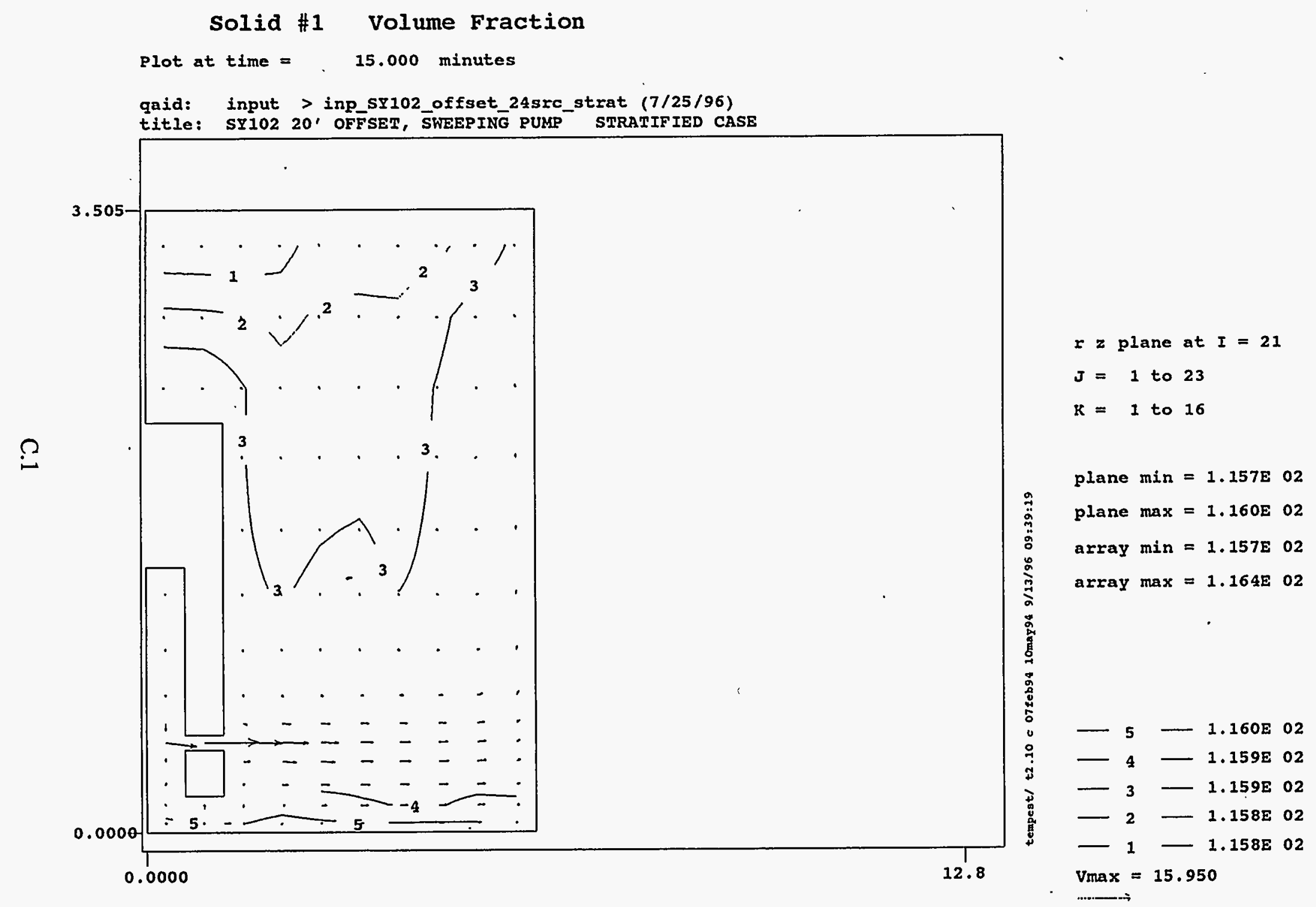

Figure C.1. Predicted Velocity $(\mathrm{m} / \mathrm{s})$ and Distribution of Solid 1 Volume Fraction (non-dimension) on Vertical Plane 21 (9 o'clock position) at 15 Simulation Minutes for the Coarser Grid Resolution Case 


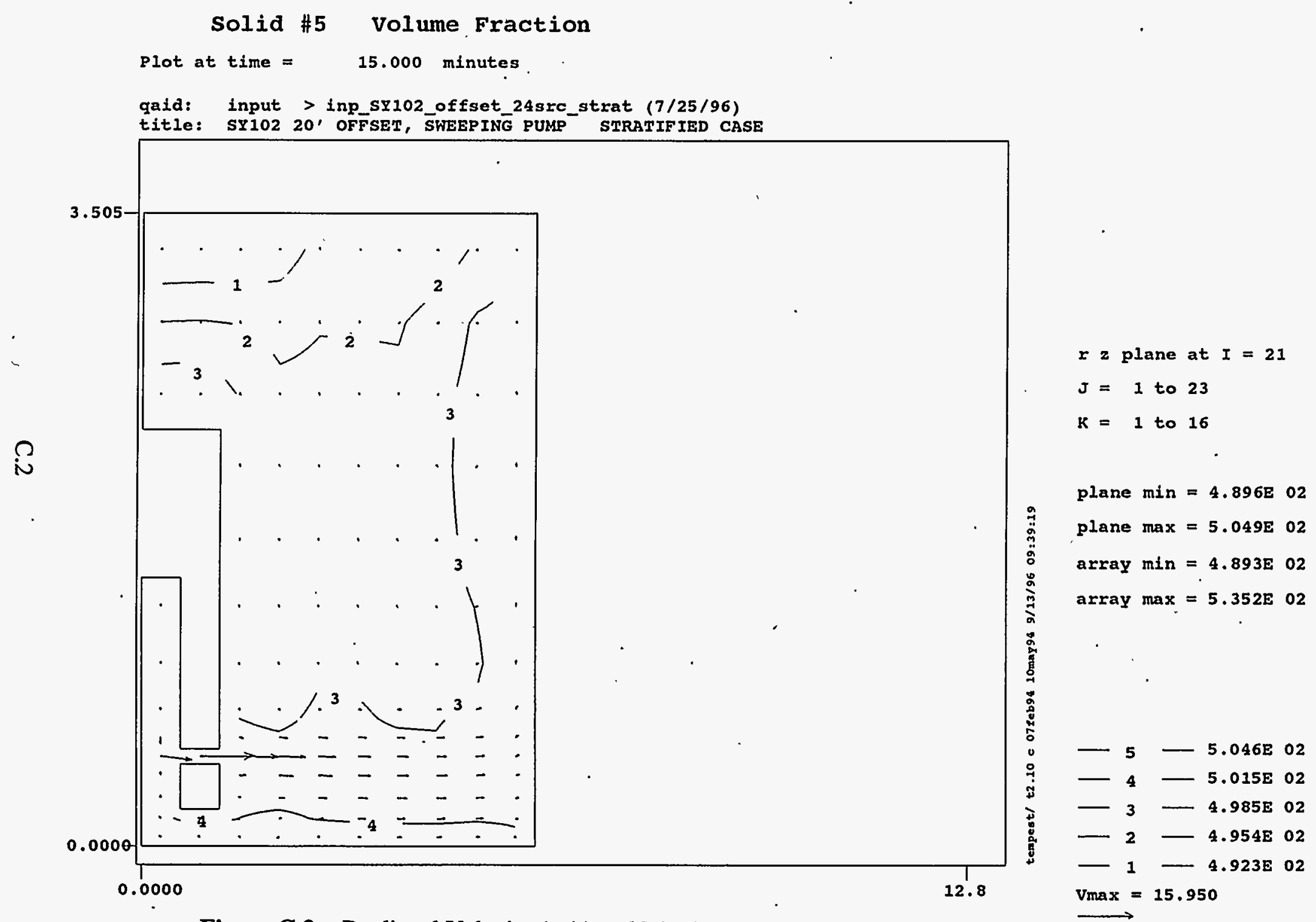

Figure C.2. Predicted Velocity $(\mathrm{m} / \mathrm{s})$ and Distribution of Solid 5 Volume Fraction on Vertical Plane 21 (9 o'clock position) at 15 Simulation Minutes for the Coarser Grid Resolution Case 


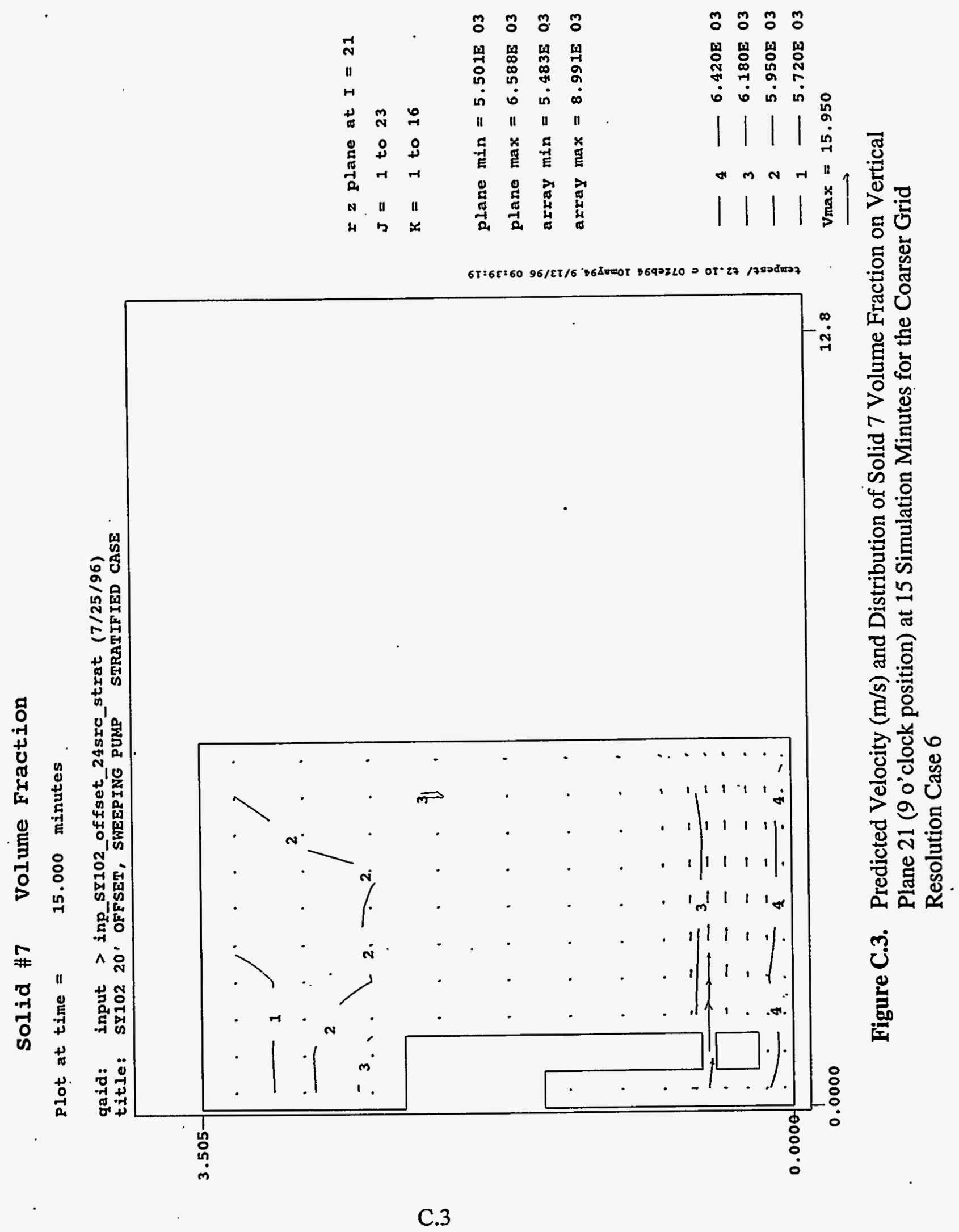




\section{Solid \#1 Volume Fraction}

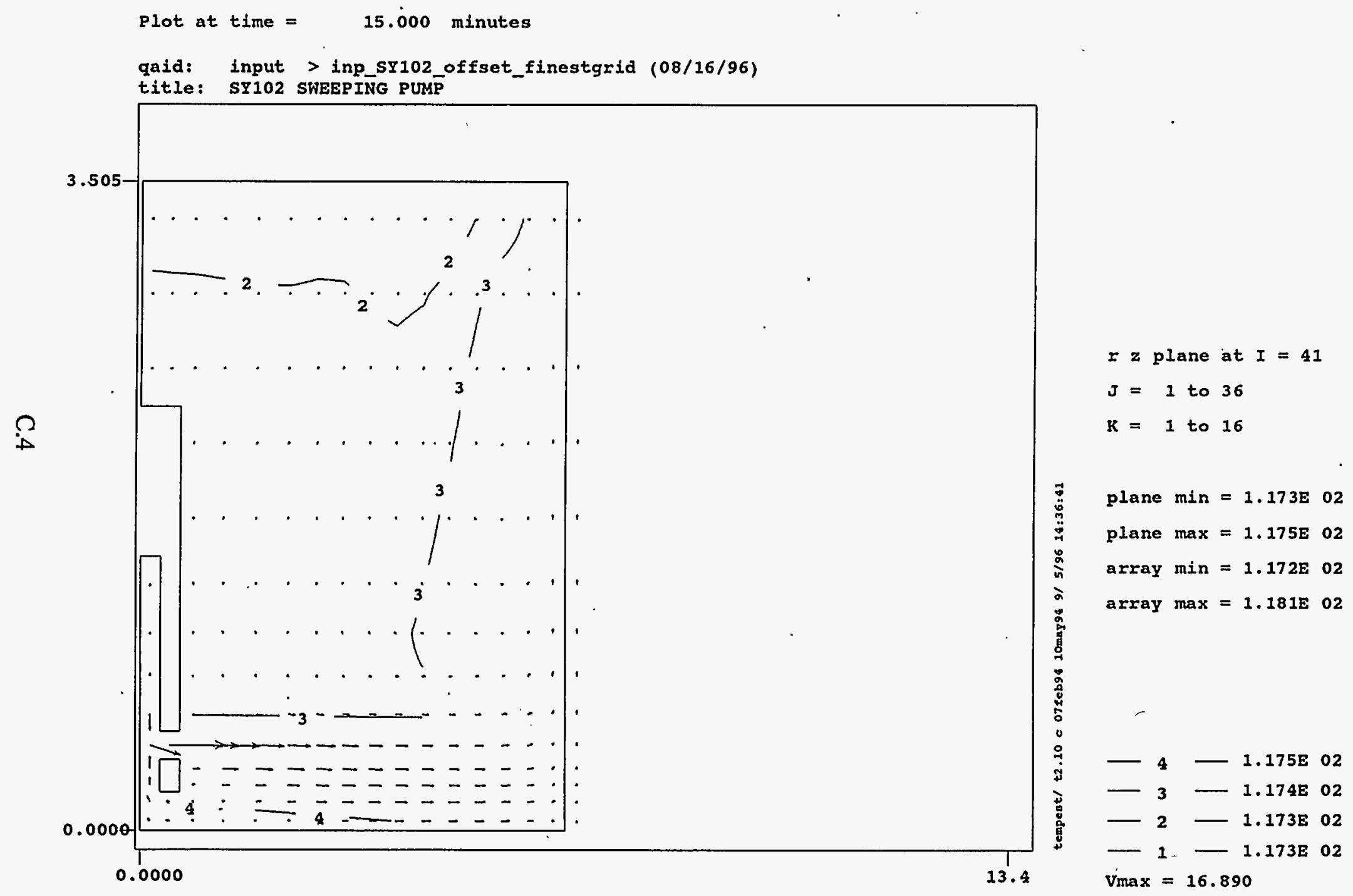

Figure C.4. Predicted Velocity $(\mathrm{m} / \mathrm{s})$ and Distribution of Solid 1 Volume Fraction on Vertical Plane 41 (9 o'clock position) at 15 Simulation Minutes for the Finer Grid Resolution Case 


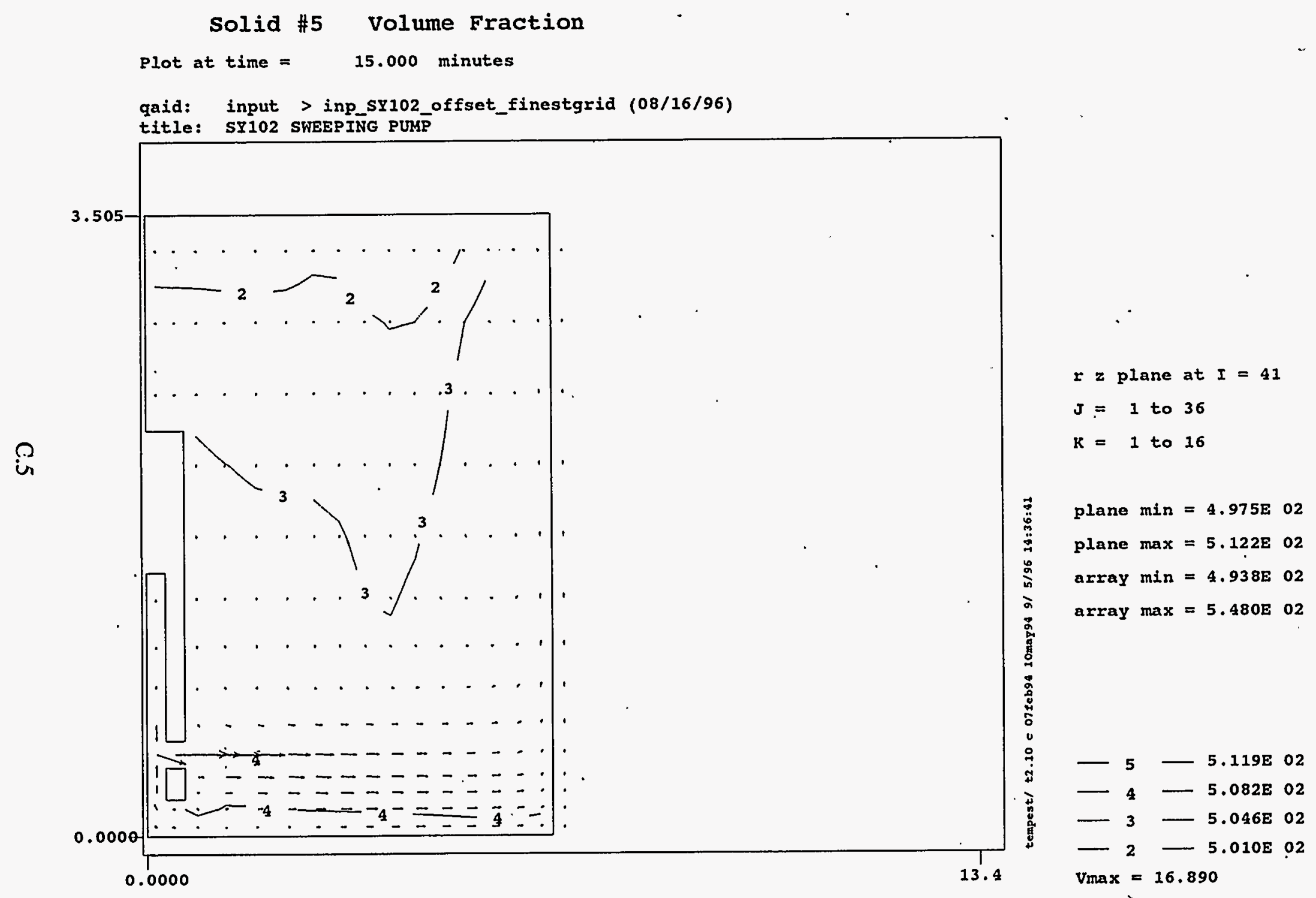

Figure C.5. Predicted Velocity $(\mathrm{m} / \mathrm{s})$ and Distribution of Solid 5 Volume Fraction on Vertical Plane 41 (9 o'clock position) at 15 Simulation Minutes for the Finer Grid

Resolution Case 


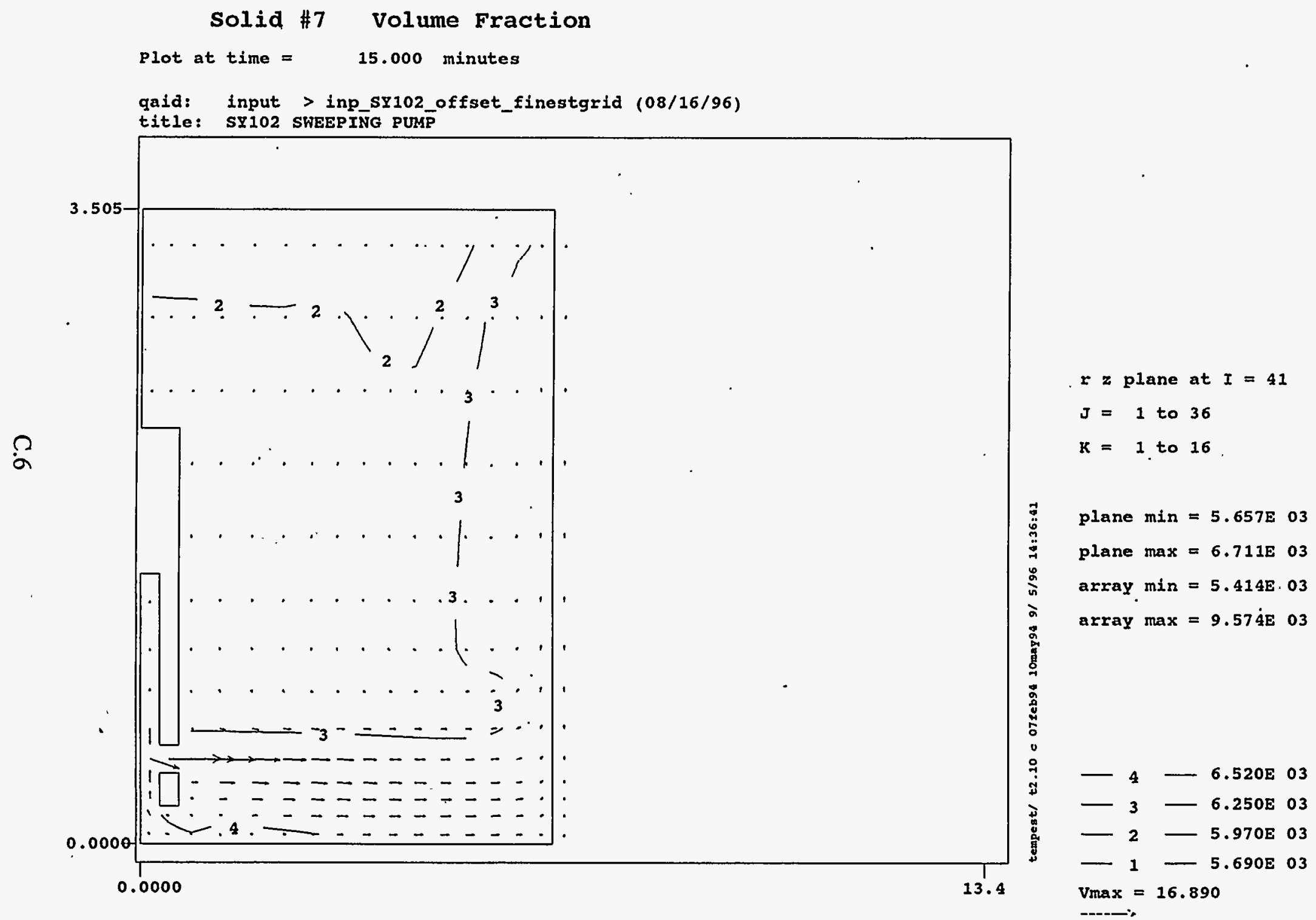

Figure C.6. Predicted Velocity $(\mathrm{m} / \mathrm{s})$ and Distribution of Solid 7 Volume Fraction on Vertical Plane (9 o'clock position) at 15 Simulation Minutes for the Finer Grid Resolution Case 


\section{Plot at time $=$ \\ qaid: input $>$ inp_sY102 offset_24src_strat (7/25/96) \\ title: SY102 20, OFFSET, SWEEPING PUMP STRATIFIED CASE \\ Solid \#7 volume Fraction}

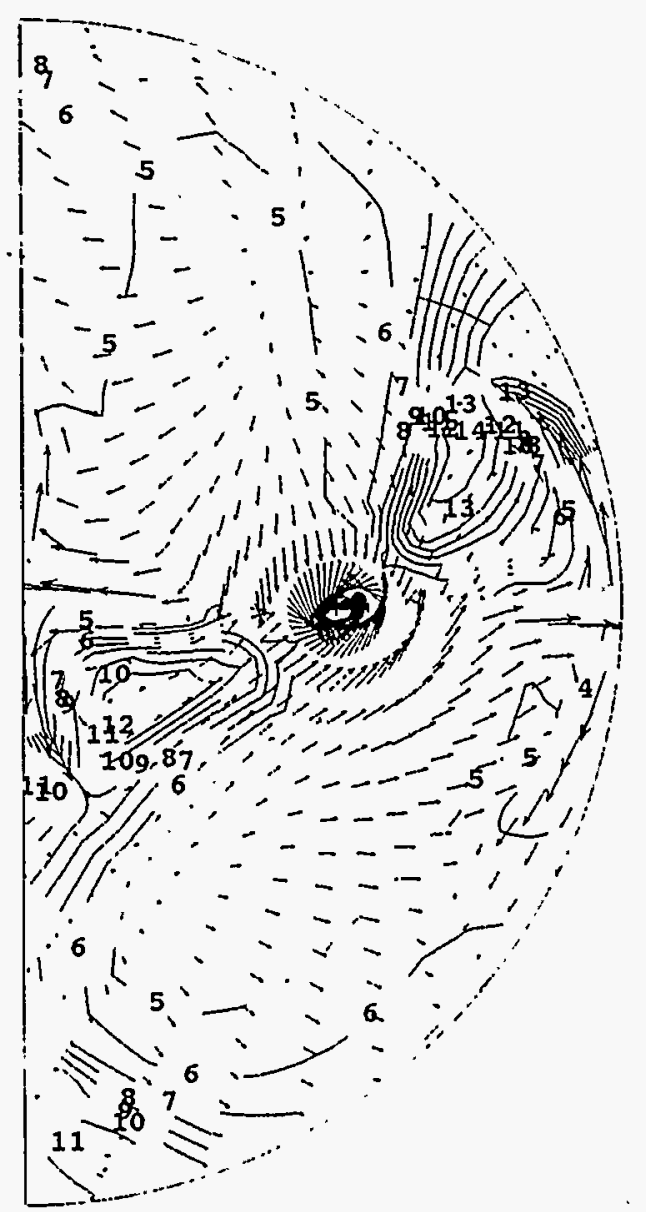

$r \times$ plane at $K=2$

$J=2$ to 23

$I=1$ to 42

plane $\min =6.360 \mathrm{E} 03$ plane $\max =8.991 \mathrm{E} 03$ array $\min =5.483 \mathrm{E} 03$ array $\max =8.991 \mathrm{E} 03$

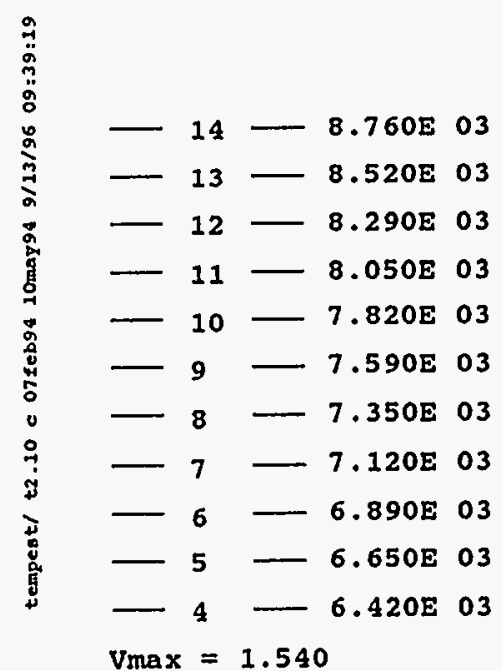

Figure C.7. Predicted Velocity $(\mathrm{m} / \mathrm{s})$ and Horizontal Distribution of Solid 7 Volume Fraction on the Tank Bottom at 15 Simulation Minutes for the Coarser Grid Resolution Case 
Plot at time $=\quad 15.000$ minutes
qaid: input $>$ inp_sY102_offset_24src_strat $(7 / 25 / 96)$
title: SY102 20, OFFSET, SWEEPING PUMP STRATIFIED CASE

Solid \#7 Volume Fraction

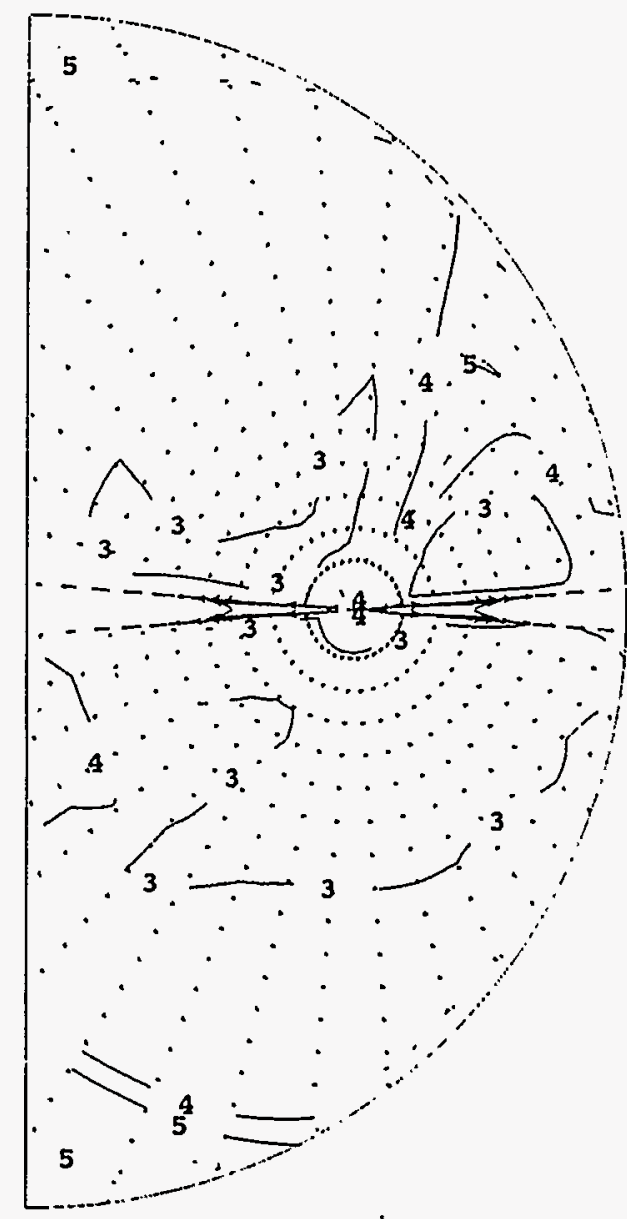

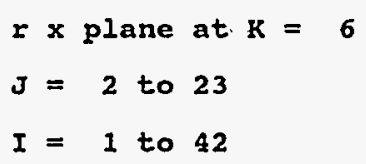

Figure C.8. Predicted Velocity $(\mathrm{m} / \mathrm{s})$ and Horizontal Distribution of Solid 7 Volume Fraction at $43 \mathrm{~cm}$ (17 inches) (height of the jet injection nozzles) above Tank Bottom at 15 Simulation Minutes for the Coarser Grid Resolution Case 
qaid: input > inp ST102 offset 24sre_strat $(7 / 25 / 96)$

title: SY102 20' OFFSEY, SWBEPING PUMP STRATIFIED CASE

\section{Solid \#7 volume Fraction}

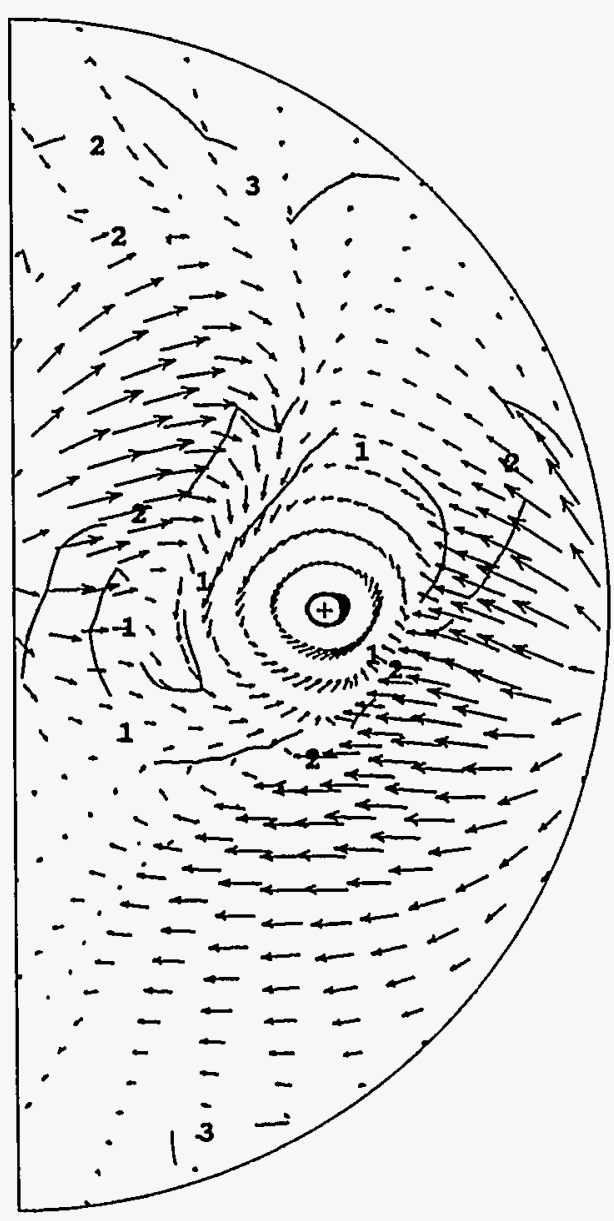

$x \times$ plane at $k=15$

$J=2$ to 23

$I=1$ to 42

plane $\min =5.483 \mathrm{~B} 03$ plane max $=6.370 \mathrm{E} 03$ array $\min =5.483 \mathrm{~B} 03$ array $\max =8.991 \mathrm{~B} \quad 03$

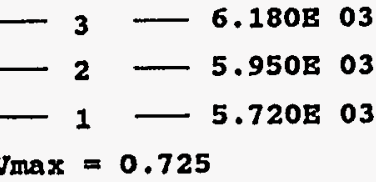

Figure C.9. Predicted Velocity $(\mathrm{m} / \mathrm{s})$ and Horizontal Distribution of Solid 7 Volume Fraction on the Tank Waste Surface ( $3.5 \mathrm{~m}$ or 138 inches above the tank bottom) at 15 Simulation Minutes for the Coarser Grid Resolution Case 
qaid: input > inp_sY102_offset_finestgrid $(08 / 16 / 96)$

title: SY102 SWEEPING PUMP

\section{Solid \#7 Volume Fraction}

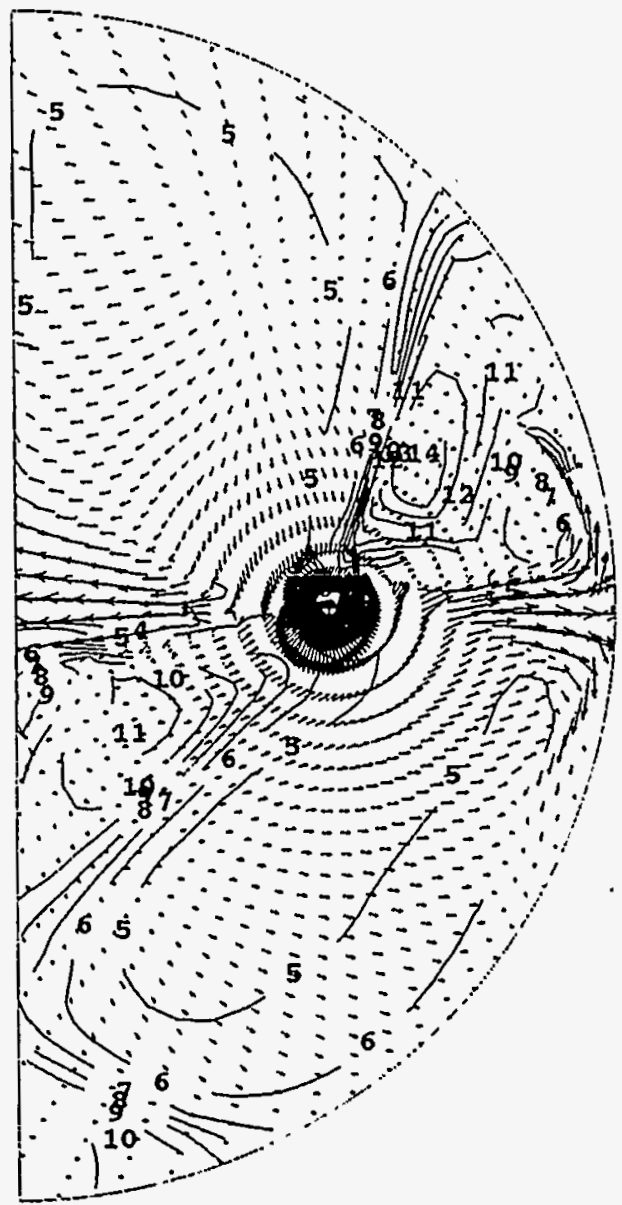

$\mathrm{x}$ plane at $\mathrm{K}=2$

$J=2$ to 36

$I \doteq 1$ to 82

plane $\mathrm{min}=6.329 \mathrm{E} 03$ plane $\max =9.574 \mathrm{E} 03$ array $\min =5.414 \mathrm{E} 03$ array $\max =9.574 \mathrm{E} 03$

Figure C.10. Predicted Velocity $(\mathrm{m} / \mathrm{s})$ and Horizontal Distribution of Solid 7 Volume Fraction on the Tank Bottom at 15 Simulation Minutes for the Finer Grid Resolution Case 


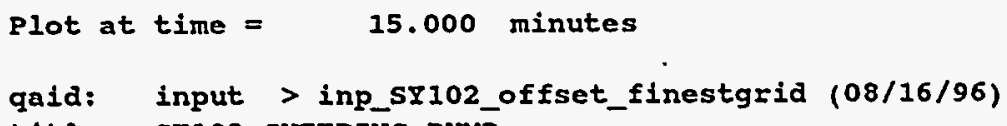

\section{Solid \#7 Volume Fraction}
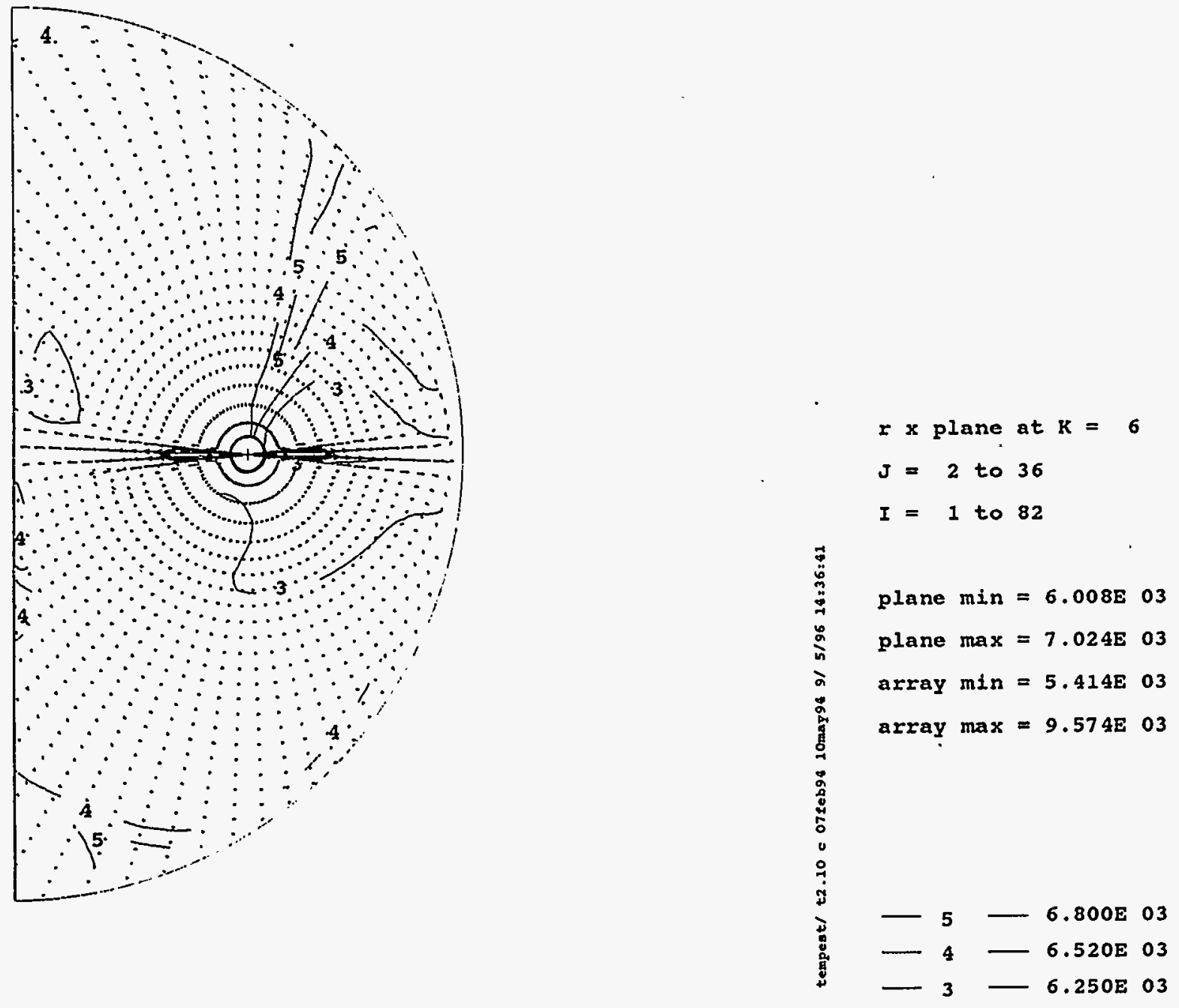

$\longrightarrow$

Figure C.11. Predicted Velocity $(\mathrm{m} / \mathrm{s})$ and Horizontal Distribution of Solid 7 Volume Fraction at $43 \mathrm{~cm}$ above Tank Bottom at 15 Simulation Minutes for the Finer Grid Resolution Case 
qaid: input > inp SY102_offset_finestgrid (08/16/96) title: SY102 SWEEPING PUMP

\section{Solid \#7 Volume Fraction}

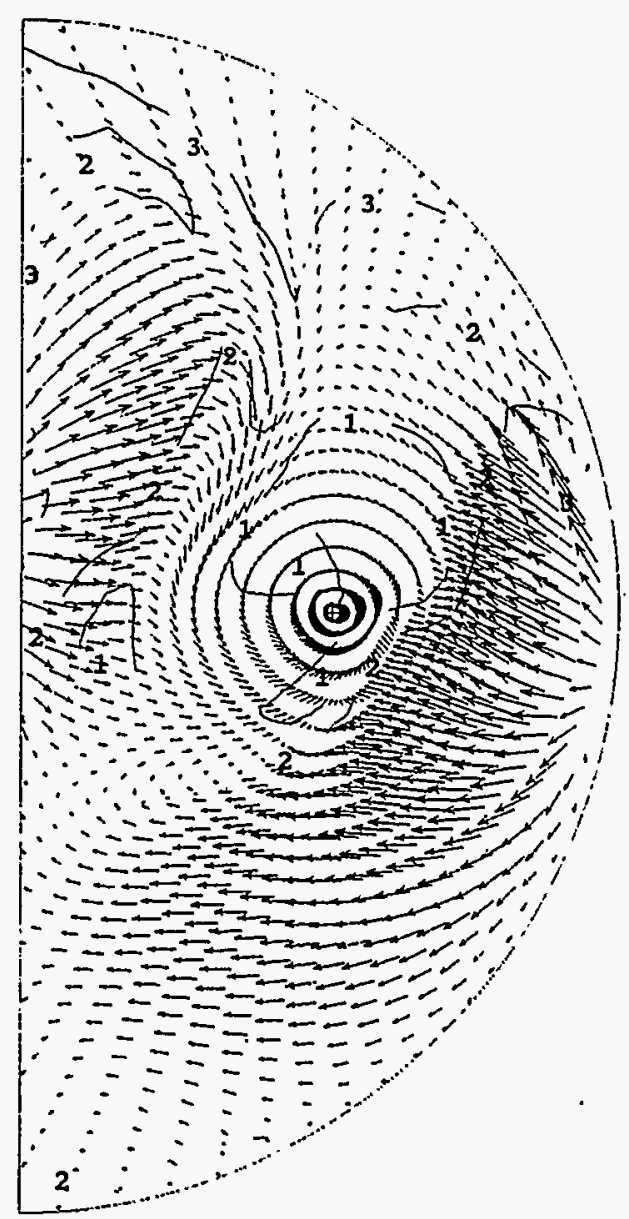

$r \times$ plane at $K=15$

$J=2$ to 36

$I=1$ to 82

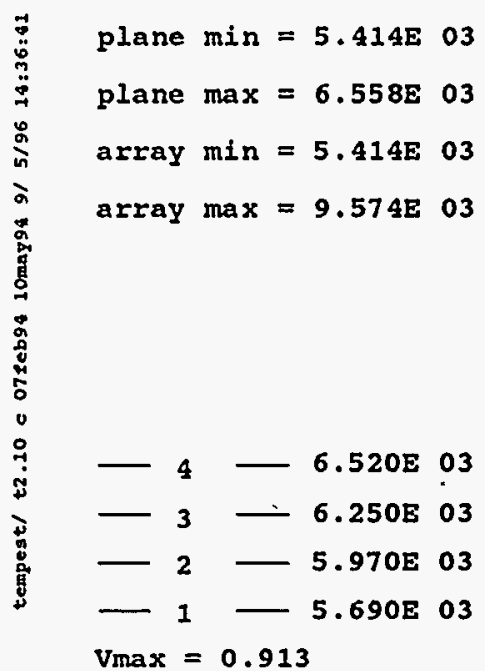

$V \max =0.913$

Figure C.12. Predicted Velocity $(\mathrm{m} / \mathrm{s})$ and Horizontal Distribution of Solid 7 Volume Fraction on the Tank Waste Surface at 15 Simulation Minutes for the Finer rind Dannlutionn Cnos 


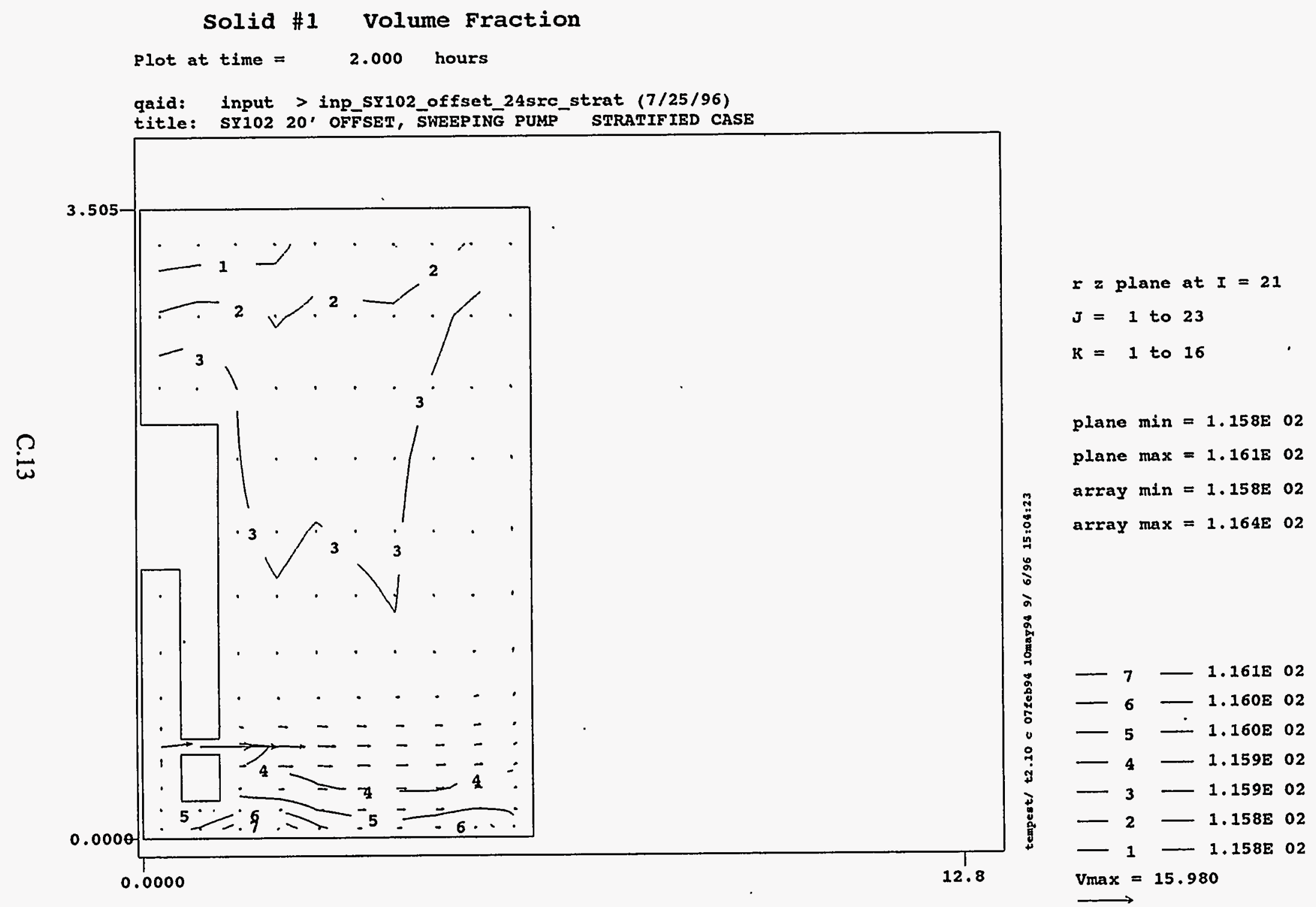

Figure C.13. Predicted Velocity $(\mathrm{m} / \mathrm{s})$ and Distribution of Solid 1 Volume Fraction on Vertical Plane 21 (9:00 Clock Position) at Two Simulation Hours for Case 1 


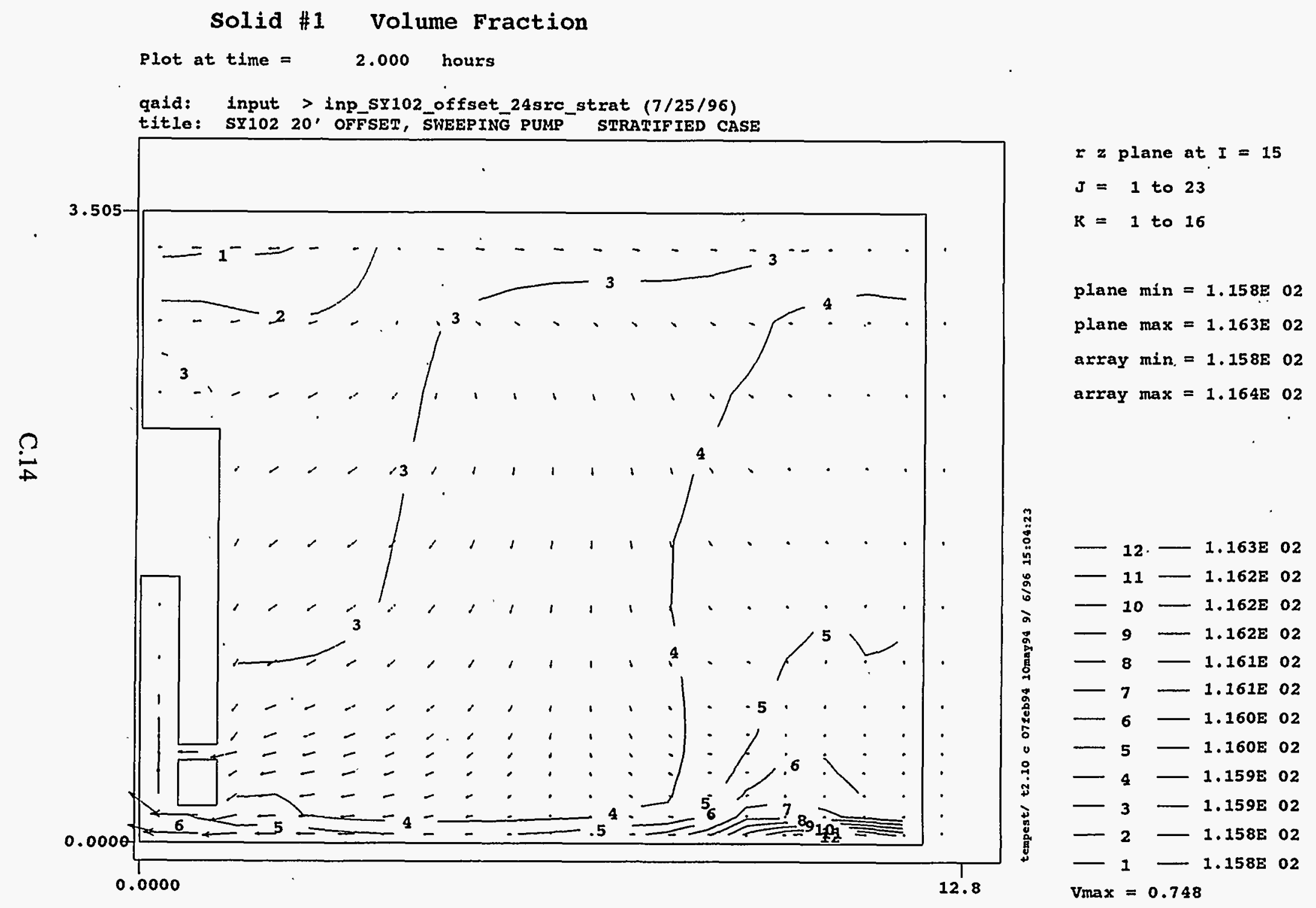

Figure C.14. Predicted Velocity $(\mathrm{m} / \mathrm{s})$ and Distribution of Solid 1 Volume Fraction on Vertical Plane 15 (11 o'clock position) at Two Simulation Hours for Case 1 


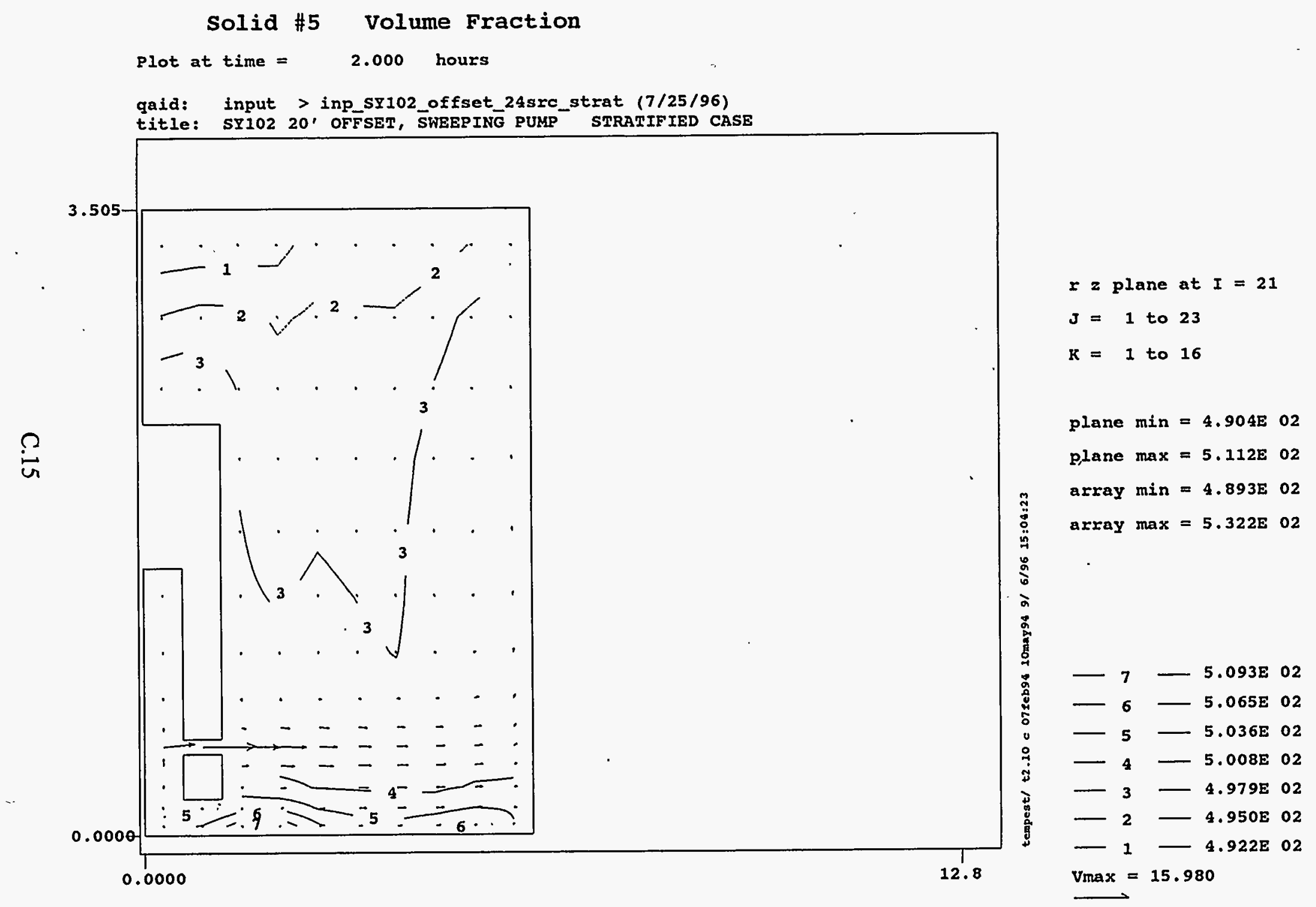

Figure C.15. Predicted Velocity $(\mathrm{m} / \mathrm{s})$ and Distribution of Solid 5 Volume Fraction on Vertical Plane 21 (9 o'clock position) at Two Simulation Hours for Case 1 


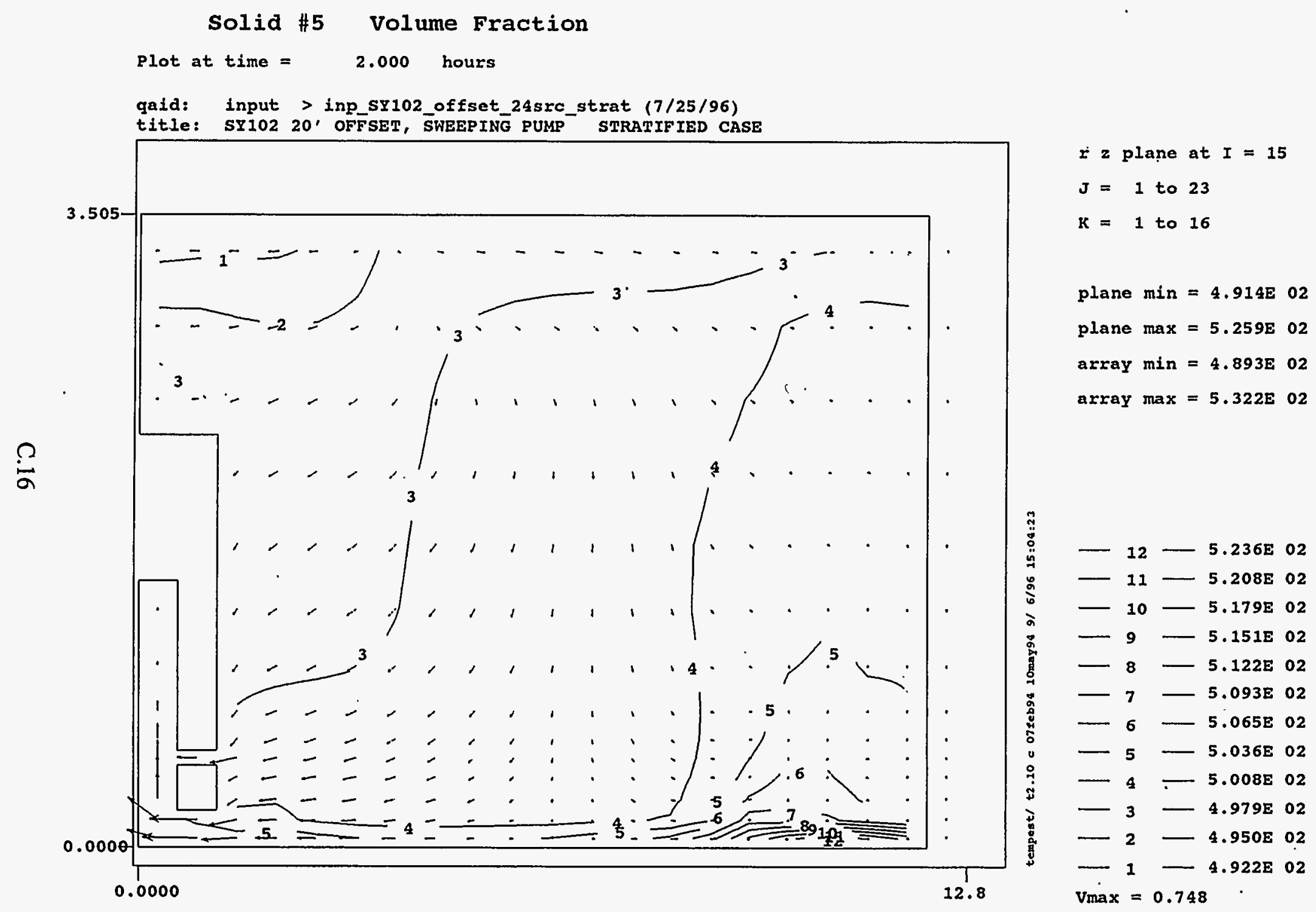

Figure C.16. Predicted Velocity $(\mathrm{m} / \mathrm{s})$ and Distribution of Solid 5 Volume Fraction on Vertical Plane 15'(11 o'clock position) at Two Simulation Hours for Case 1 


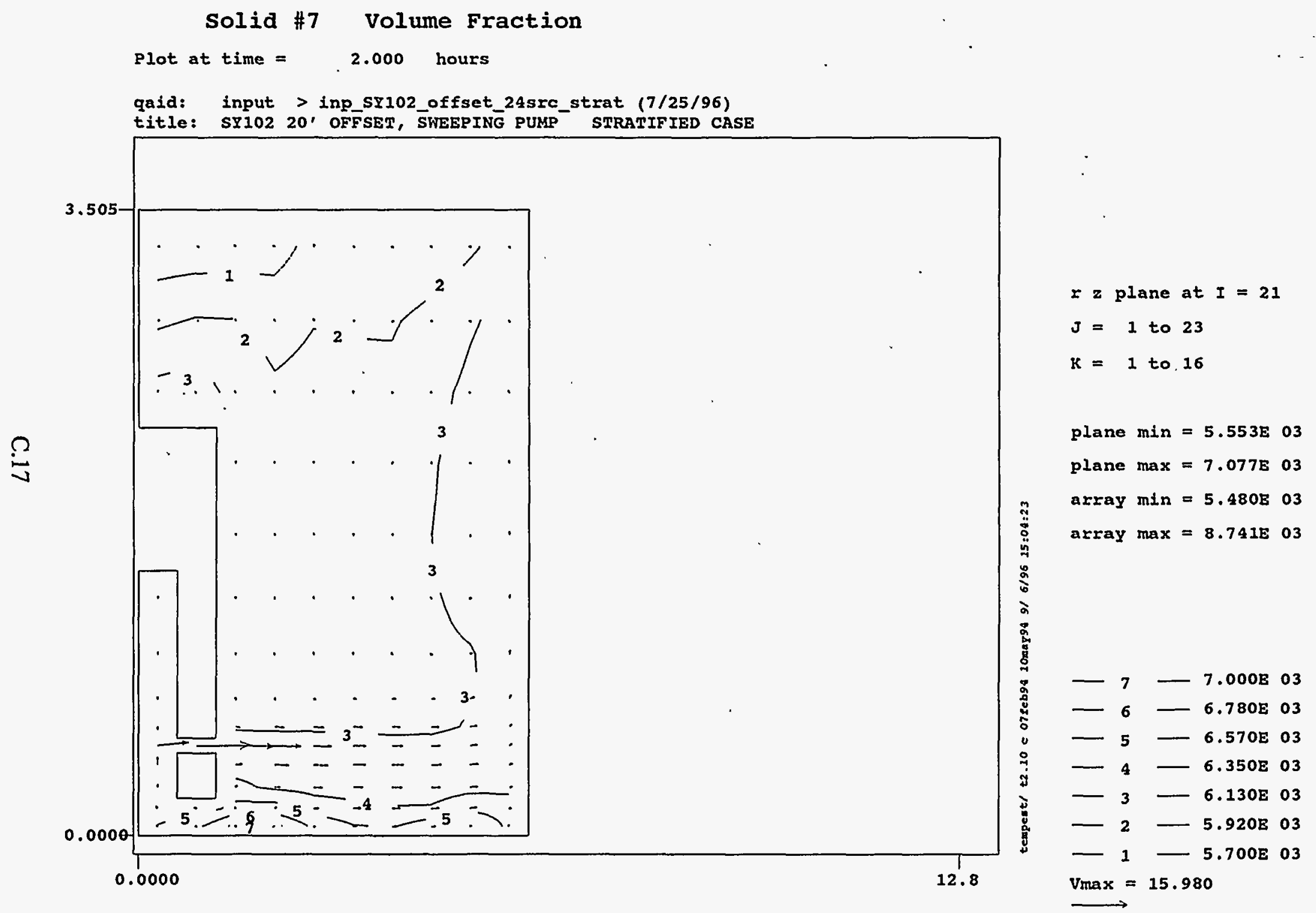

Figure C.17. Predicted Velocity $(\mathrm{m} / \mathrm{s})$ and Distribution of Solid 7 Volume Fraction on Vertical Plane 21 (9 o'clock position) at Two Simulation Hours for Case 1 


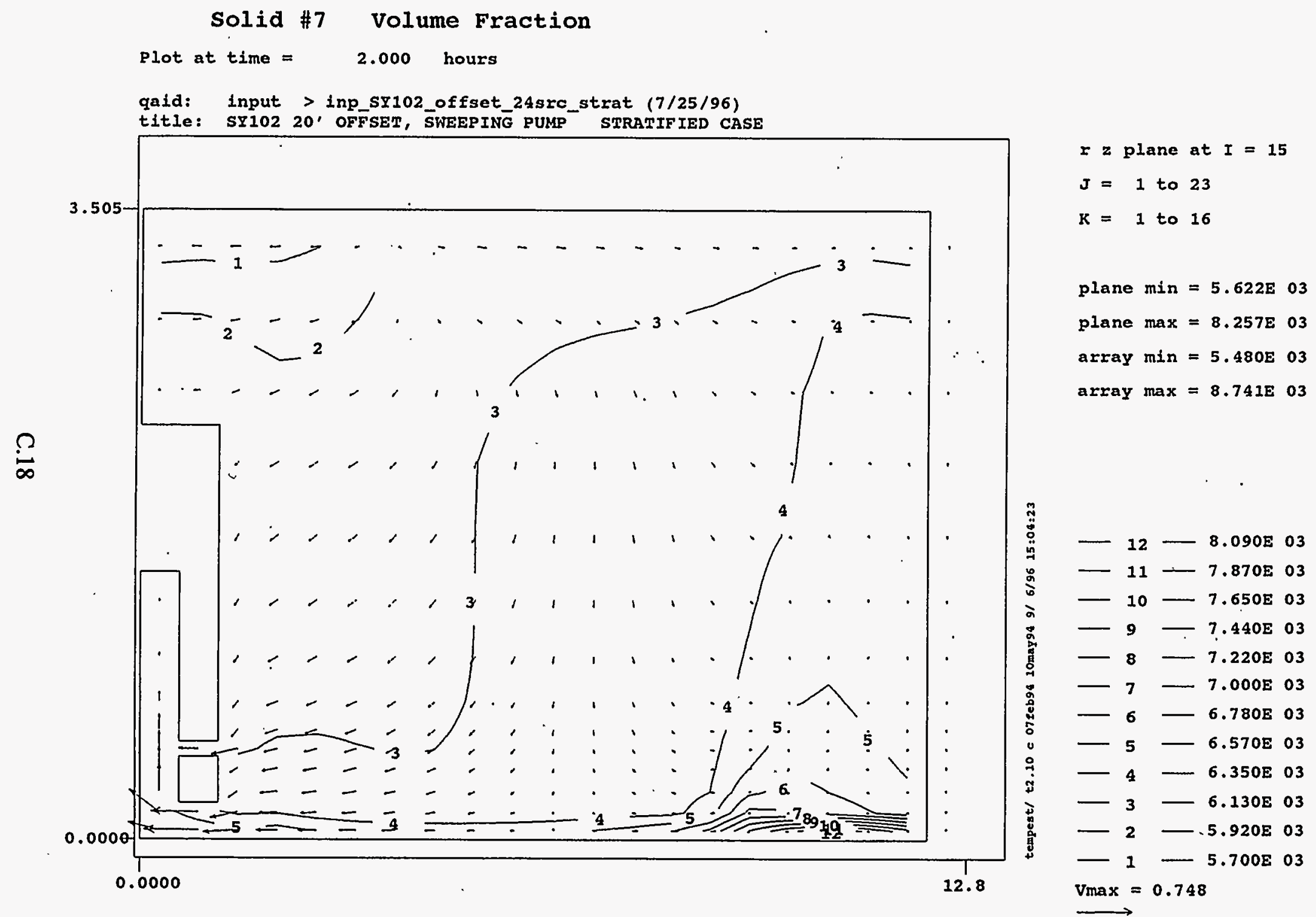

Figure C.18. Predicted Velocity $(\mathrm{m} / \mathrm{s})$ and Distribution of Solid. 7 Volume Fraction on Vertical Plane 15 (11 o'clock position) at Two Simulation Hours for Case 1 
Plot at time $=\quad 2.000$ hours

qaid: input > inp_SY102_offset_24src_strat $(7 / 25 / 96)$

title: ST102 20' OFFSET, SWEEPING PUMP STRATIFIED CASE

\section{Solid \#1 volume Fraction}

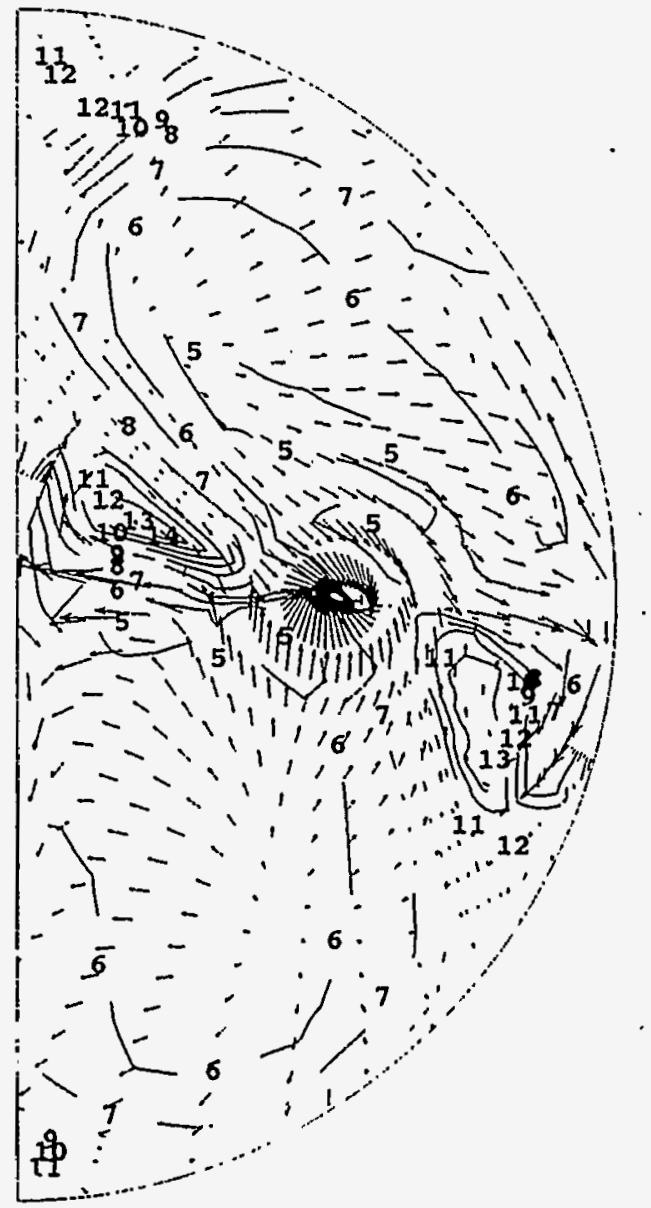

$r$ plane at $k=2$

$J=2$ to 23

$I=1$ to 42

plane $\min =1.160 \mathrm{E} 02$ plane $\max =1.164 \mathrm{E} 02$ array $\min =1.158 \mathrm{E} 02$ array $\max =1.164 \mathrm{E} 02$

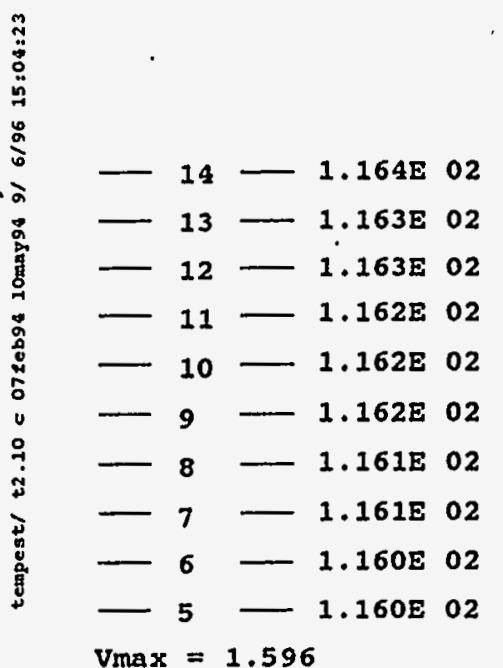

Figure C.19. Predicted Velocity $(\mathrm{m} / \mathrm{s})$ and Horizontal Distribution of Solid 1 Volume Fraction on the Tank Bottom at Two Simulation Hours for Case 1 


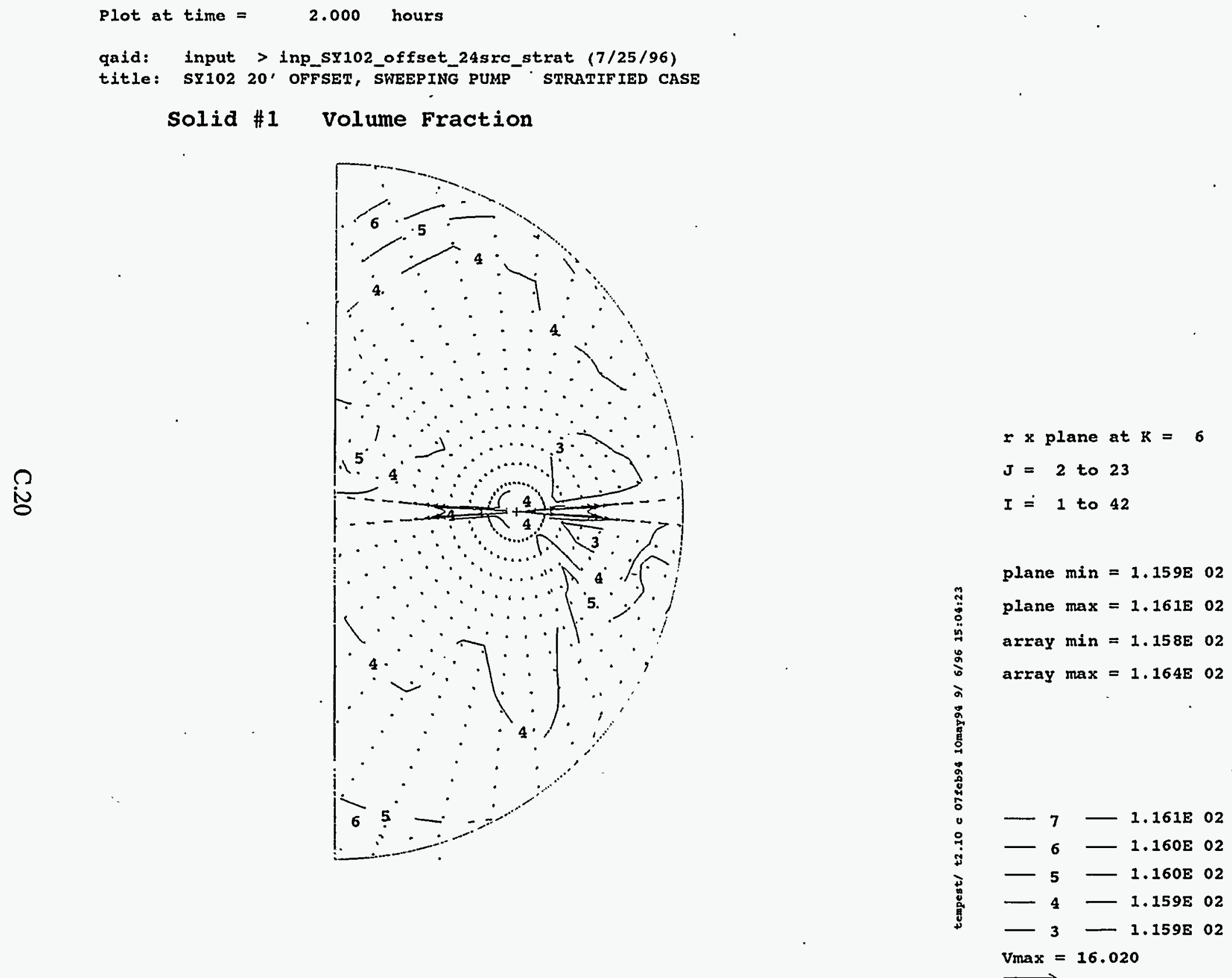

Figure C.20. Predicted Velocity $(\mathrm{m} / \mathrm{s})$ and Horizontal Distribution of Solid 1 Volume Fraction at $43 \mathrm{~cm}$ (17 inches) (height of the jet injection nozzles) above Tank Bottom at Two Simulation Hours for Cace 1 
qaid: input $>$ inp SY102 offset 24src_strat $(7 / 25 / 96)$

title: SY102 20, OFFSET, SWEEPING PUMP STRATIFIED CASE

\section{Solid \#1 Volume Fraction}

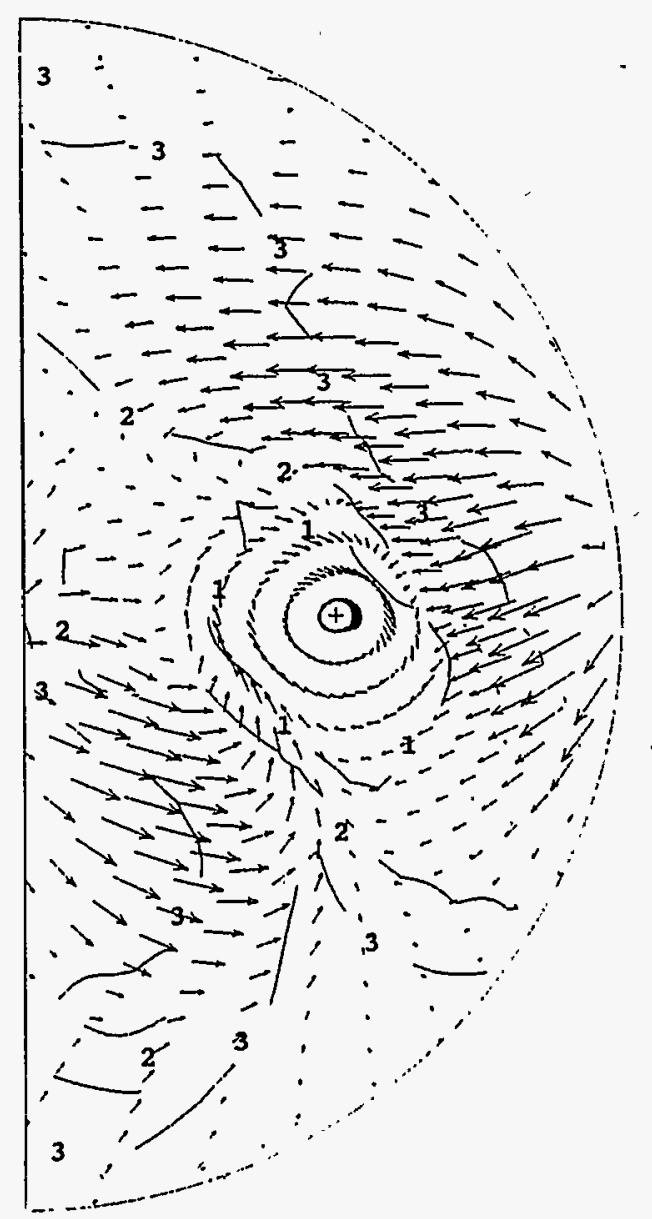

$r \times$ plane at $k=15$

$J=2$ to 23

$I=I$ to 42

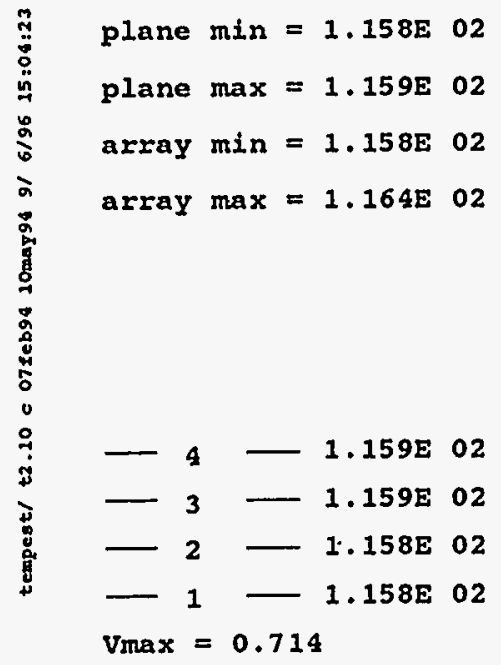

$v_{\max }=0.714$

Figure C.21. Predicted Velocity $(\mathrm{m} / \mathrm{s})$ and Horizontal Distribution of Solid 1 Volume Fraction on the Tank Waste Surface ( $3.5 \mathrm{~m}$ or 138 inches above the tank bottom) at Two Simulation Hours for Case 1 
Plot at time $=2.000$ hours
qaid: input > inp_SY102_offset_24src_strat (7/25/96)
title: SY102 20, OFFSET, SWEEPING PUMP STRATIFIED CASE

Solid \#5 Volume Fraction

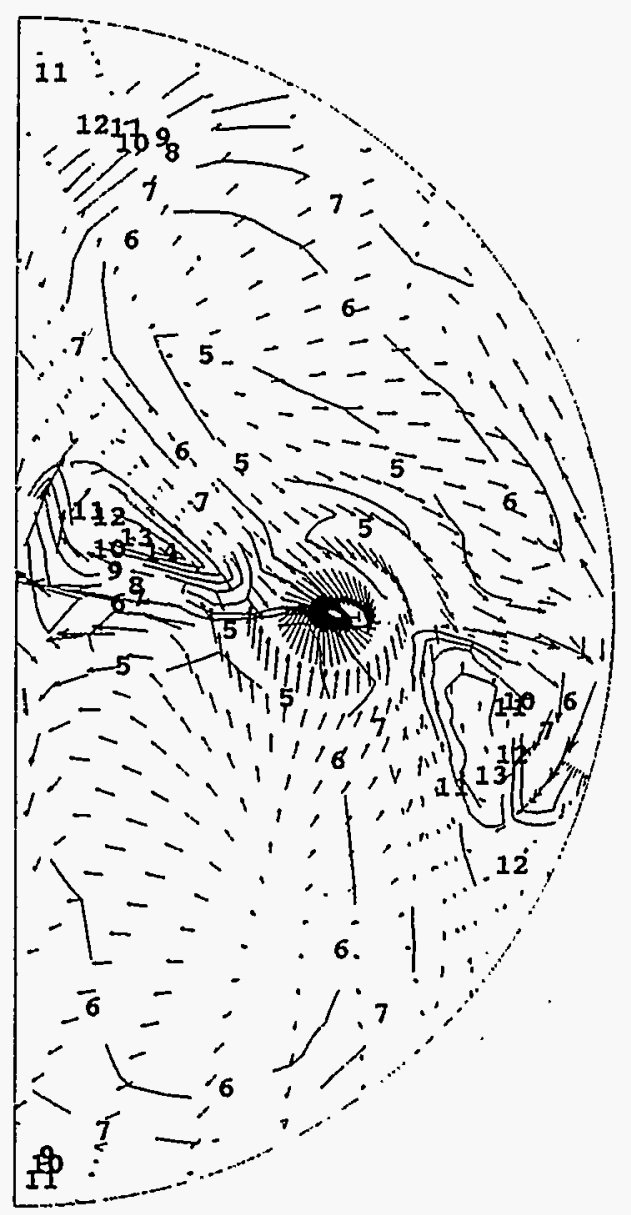

I $x$ plane at $k=2$

$J=2$ to 23

$I=1$ to 42

plane $\min =5.019 \mathrm{E} 02$ plane $\max =5.322 \mathrm{E} 02$ array $\min =4.893 \mathrm{E} 02$ array $\max =5.322 \mathrm{E} 02$

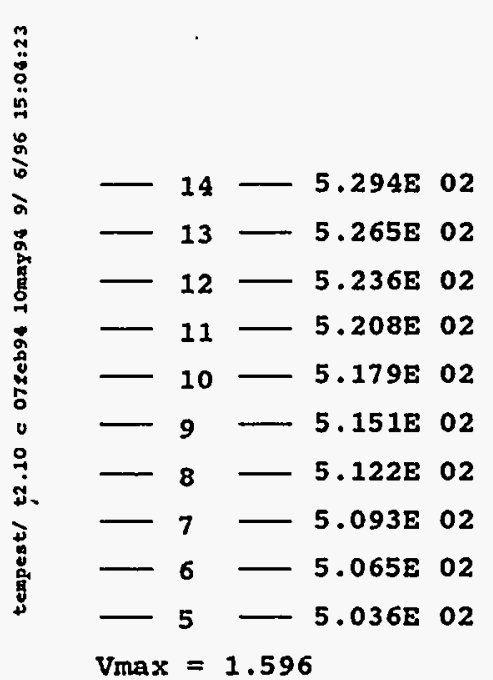

Figure C.22. Predicted.Velocity $(\mathrm{m} / \mathrm{s})$ and Horizontal Distribution of Solid 5 Volume Fraction on the Tank Bottom at Two Simulation Hours for Case 1 
Plot at time $=\quad 2.000$ hours
qaid: input $>$ inp_SY102_offset_24src_strat $(7 / 25 / 96)$
title: SY102 20, OFFSET, SWEEPING PUMP STRATIFIED CASE

Solid \#5 volume Fraction

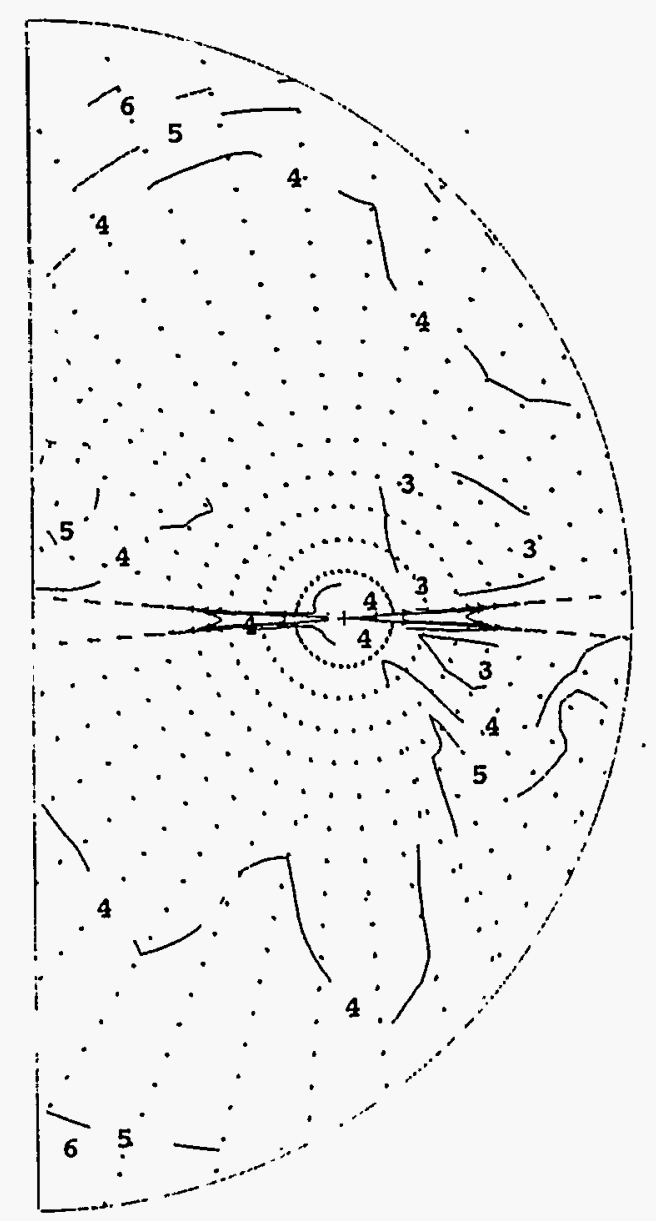

$x$ plane at $K=6$

$J=2$ to 23

$I=1$ to 42

plane min $=4.966 \mathrm{E} 02$ plane $\max =5.096 \mathrm{E} 02$ array $\min =4.893 \mathrm{E} 02$ array $\max =5.322 \mathrm{E} 02$

$-7-5.093 \mathrm{E} 02$

$-6-5.065 \mathrm{E} 02$

- $5-5.036 \mathrm{E} 02$

$-4-5.008 \mathrm{E} 02$

- $3-4.979 \mathrm{E} 02$

$V \max =16.020$

Figure C.23. Predicted Velocity $(\mathrm{m} / \mathrm{s})$ and Horizontal Distribution of Solid 5 Volume Fraction at $43 \mathrm{~cm}$ (17 inches) (height of the jet injection nozzles) above Tank Bottom at Two Simulation Hours for Case 1 
qaid: input > Inp_sY102_offset_24src_strat (7/25/96)

title: SY102 20' OFFSET, SWEEPING PUMP STRATIFIED CASE

\section{Solid \#5 Volume Fraction}

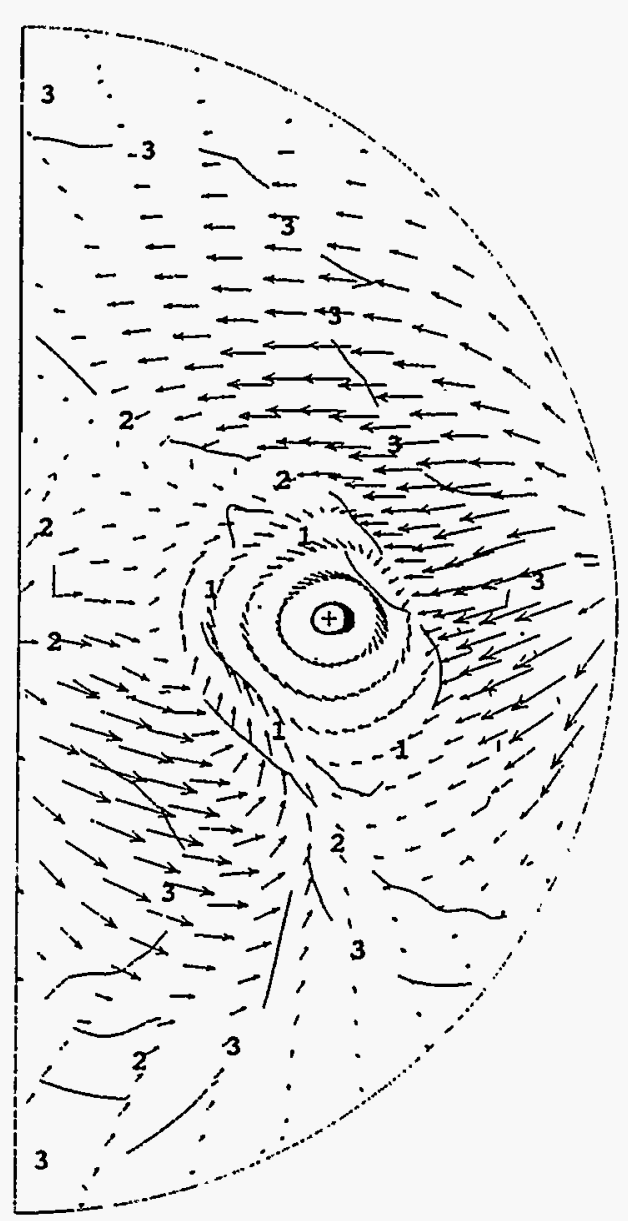

r plane at $k=15$

$J=2$ to 23

$I=1$ to 42

plane $\min =4.893 \mathrm{E} 02$

plane $\max =5.011 E 02$

array $\min =4.893 \mathrm{E} 02$

array $\max =5.322 \mathrm{E} 02$

$-4-5.008 \mathrm{E} 02$

$3-4.979 \mathrm{E} 02$

- $2-4.950 \mathrm{E} 02$

$-1-4.922 \mathrm{E} 02$

$V_{\max }=0.714$

Figure C.24. Predicted Velocity $(\mathrm{m} / \mathrm{s})$ and Horizontal Distribution of Solid 5 Volume Fraction on the Tank Waste Surface ( $3.5 \mathrm{~m}$ or 138 inches above the tank bottom) at Two Simulation Hourc for Cace 1 
qaid: input > inp_sI102_offset_24src_strat $(7 / 25 / 96)$

title: SY102 $20^{\circ}$ OFFSET, SWEEPING PUMP STRATIFIED, CASE

\section{Solid \#7 Volume Fraction}
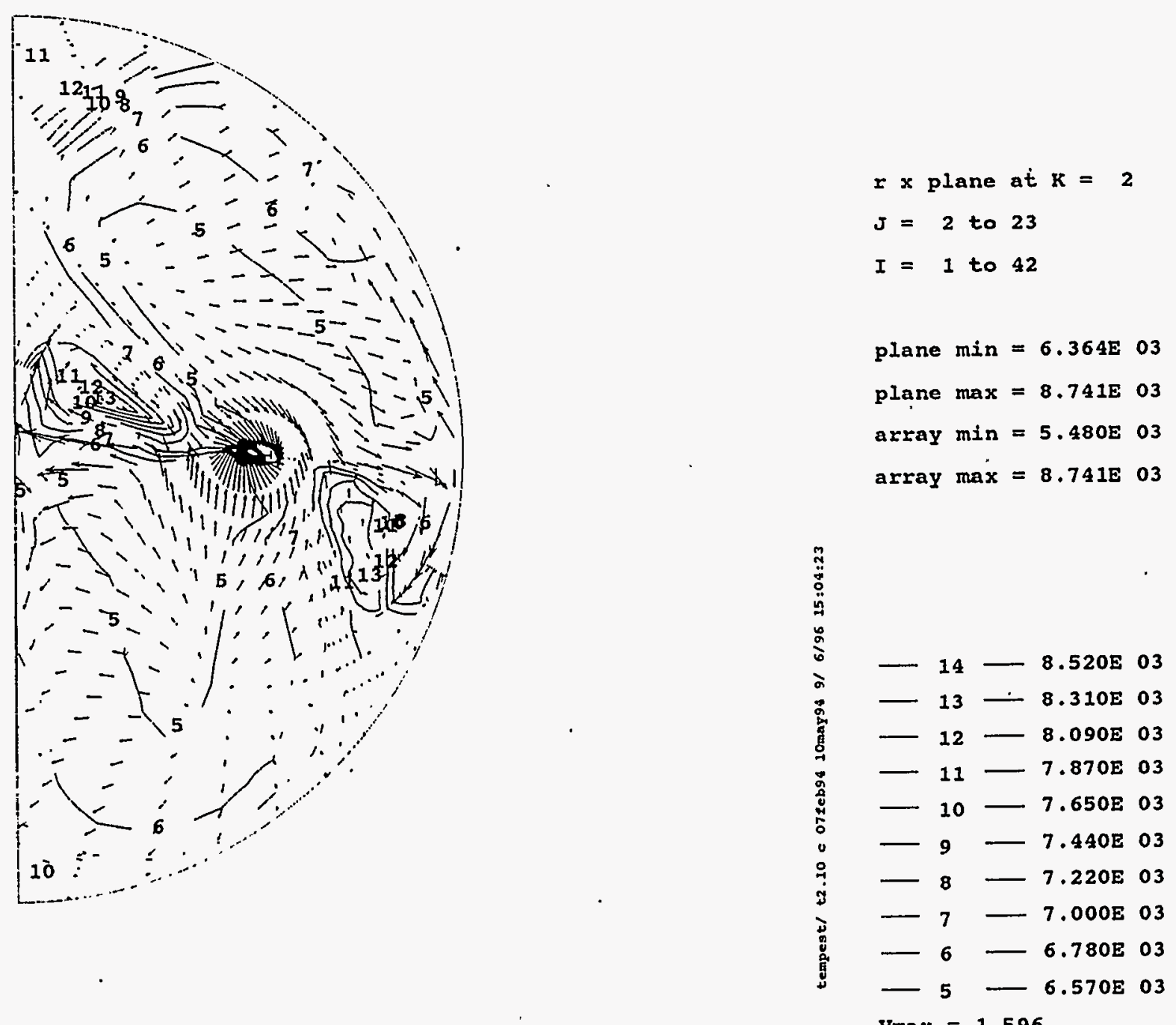

$V \max =1.596$

Figure C.25. Predicted Velocity $(\mathrm{m} / \mathrm{s})$ and Horizontal Distribution of Solid 7 Volume Fraction on the Tank Bottom at Two Simulation Hours for Case 1 


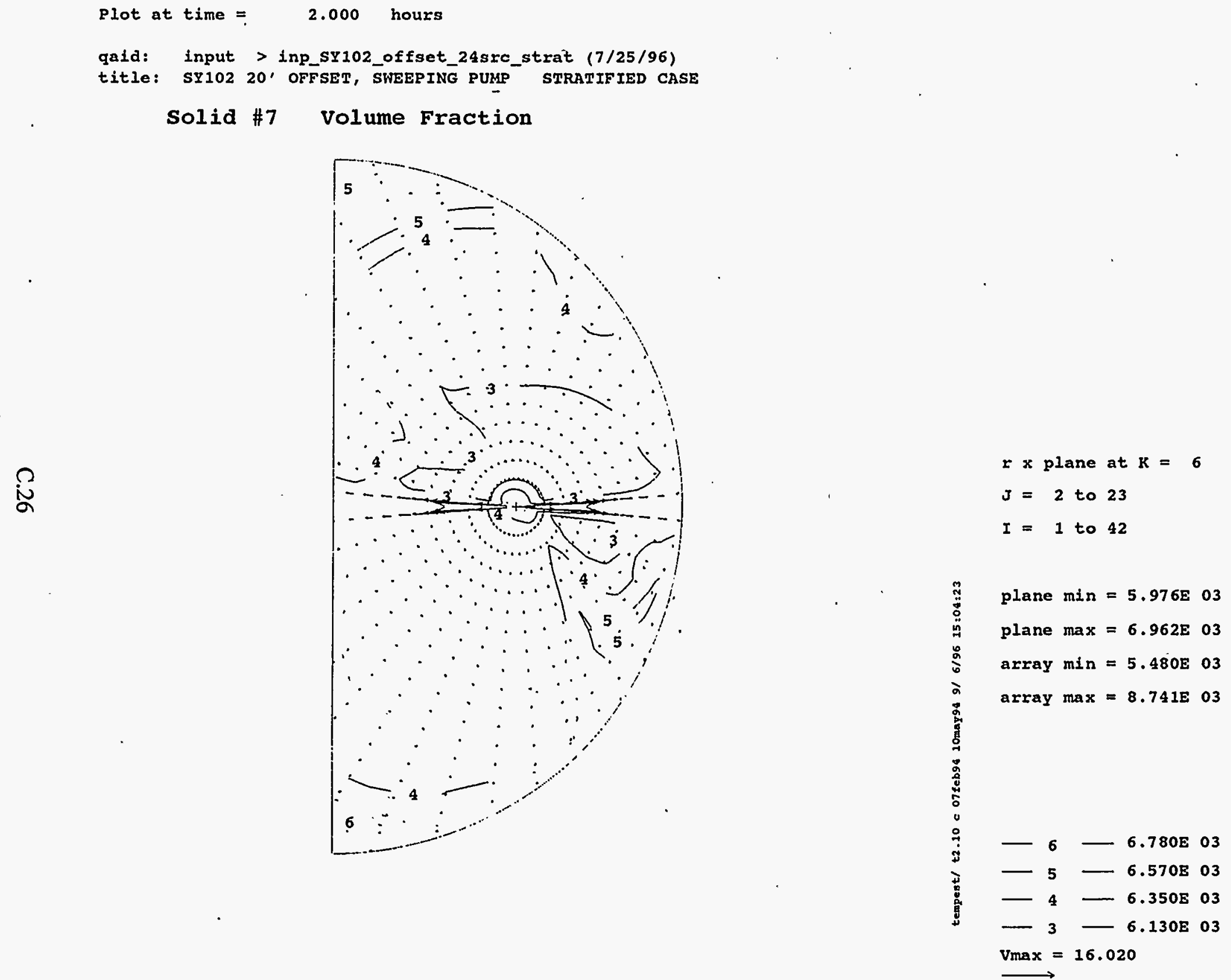

Figure C.26. Predicted Velocity $(\mathrm{m} / \mathrm{s})$ and Horizontal Distribution of Solid 7 Volume Fraction at $43 \mathrm{~cm}$ (17 inches) (height of the jet injection nozzles) above Tank Bottom at Two Simulation Hours for Case 1 
qaid: input > inp SY102 offset_24sre strat (7/25/96)

title: SY102 20' OFFSET, SWEEPING PUMP STRATIFIED CASE

\section{Solid \#7 Volume Fraction}

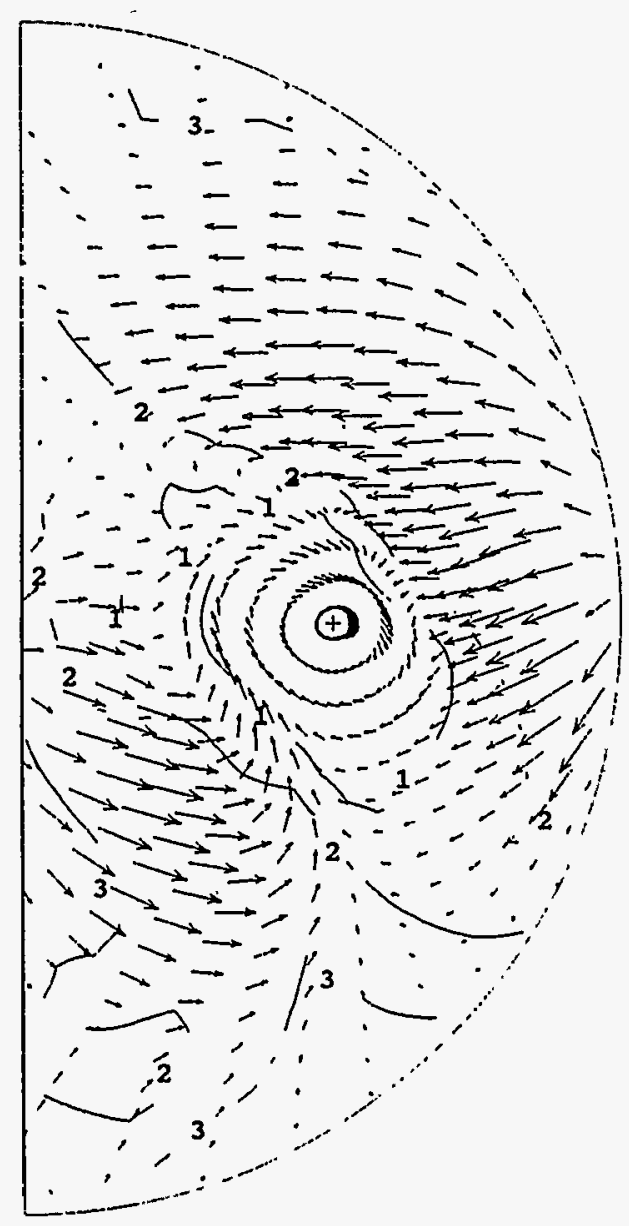

$r \times$ plane at $k=15$

$J=2$ to 23

$I=1$ to 42

plane $\min =5.480 \mathrm{E} 03$

plane $\max =6.308 \mathrm{E} 03$

array $\min =5.480 \mathrm{E} 03$

array $\max =8.741 \mathrm{E} 03$

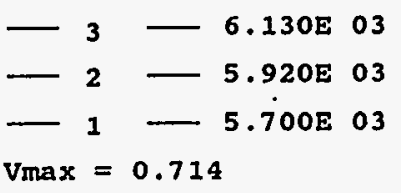

$V_{\max }=0.714$

Figure C.27. Predicted Velocity $(\mathrm{m} / \mathrm{s})$ and Horizontal Distribution of Solid 7 Volume Fraction on the Tank Waste Surface ( $3.5 \mathrm{~m}$ or 138 inches above the tank bottom) at Two Simulation Hours for Case 1 
Plot at time $=0.000$ hours
qaid: input > inp_SY102_offset_24src_strat $(7 / 25 / 96)$
title: SY102 20, OFFSET, SWEEPING PUMP STRATIFIED CASE

Solid \#1 Volume Fraction
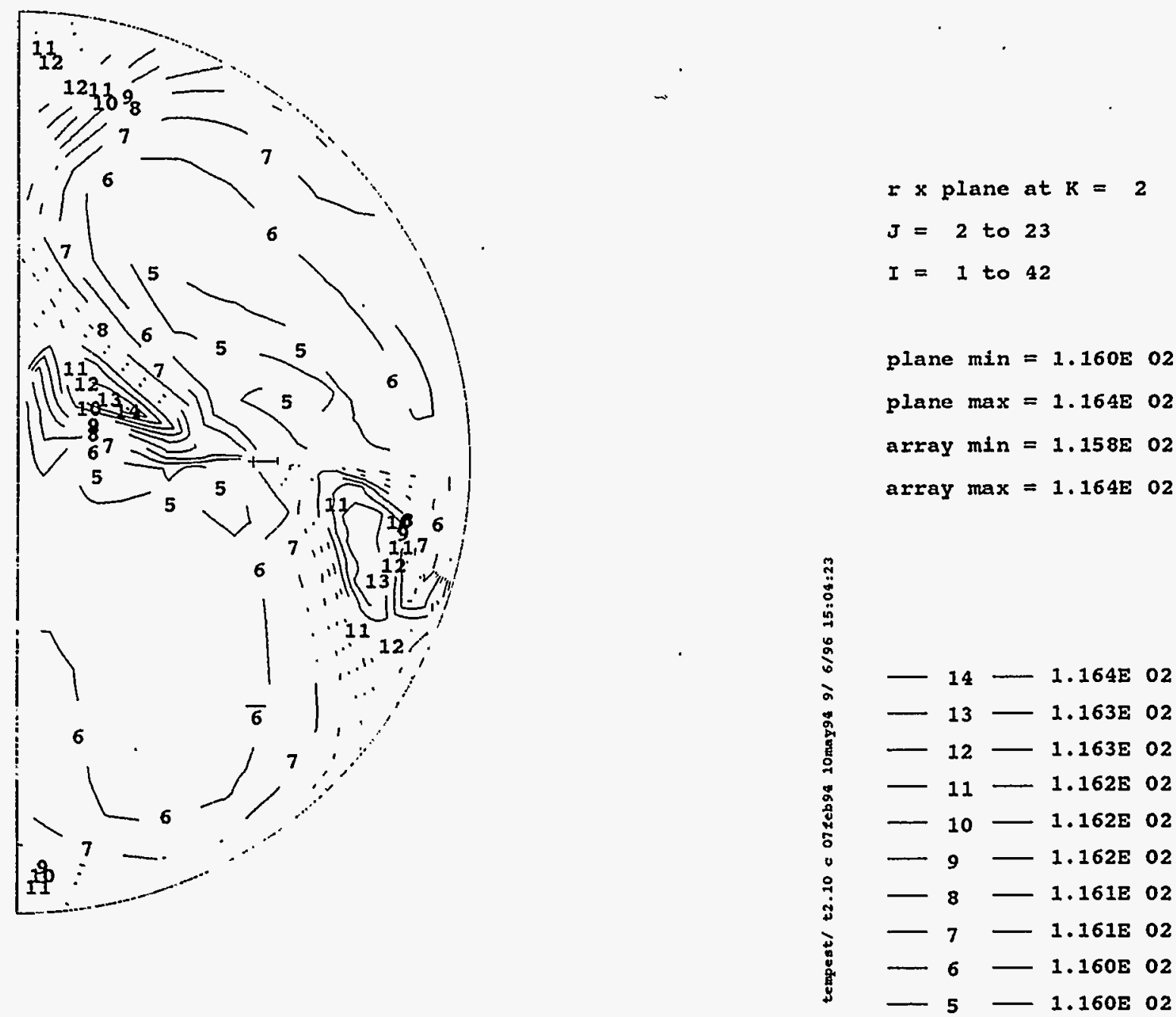

Figure C.28. Predicted Horizontal Distribution of Solid 1 Volume Fraction on the Tank Bottom at Two Simulation Hours for Case 1 
qaid: input > inp_sY102_offset_24src_strat (7/25/96)

title: SY102 20' OFFSET, SWEEPING PUMP STRATIFIED CASE

\section{Solid \#2 volume Fraction}

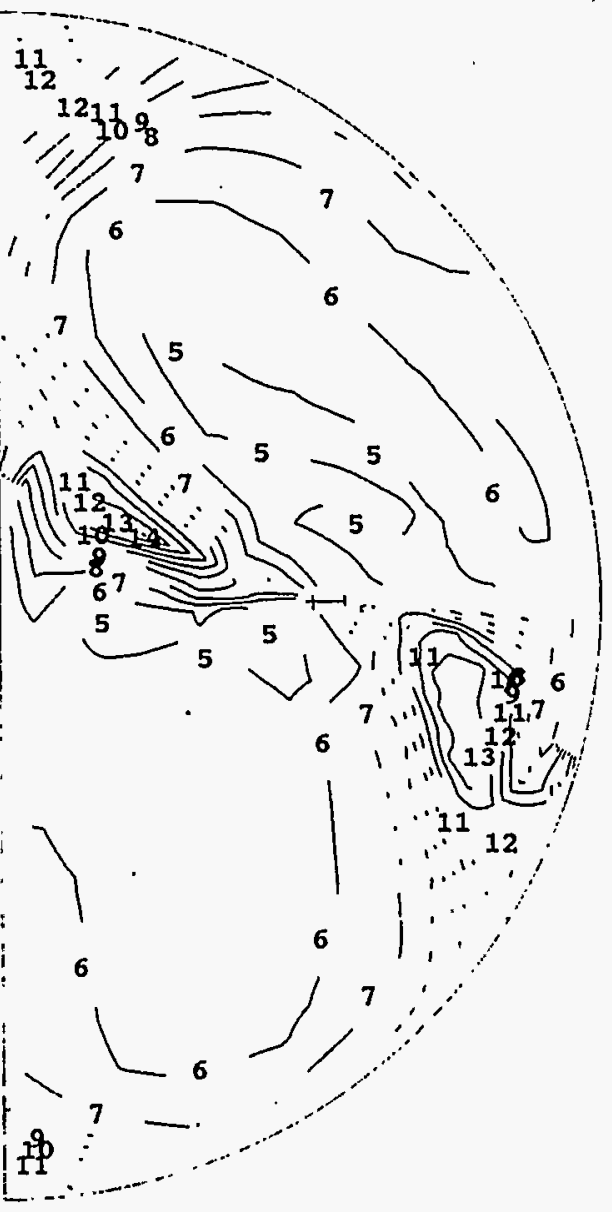

$r \times$ plane at $\mathrm{k}=2$

$J=2$ to 23

$I=1$ to 42

plane $\min =1.321 \mathrm{E} 02$ plane max $=1.336 \mathrm{E}$ 02 array $\min =1.314 \mathrm{E} 02$ axray $\max =1.336 \mathrm{E} 02$

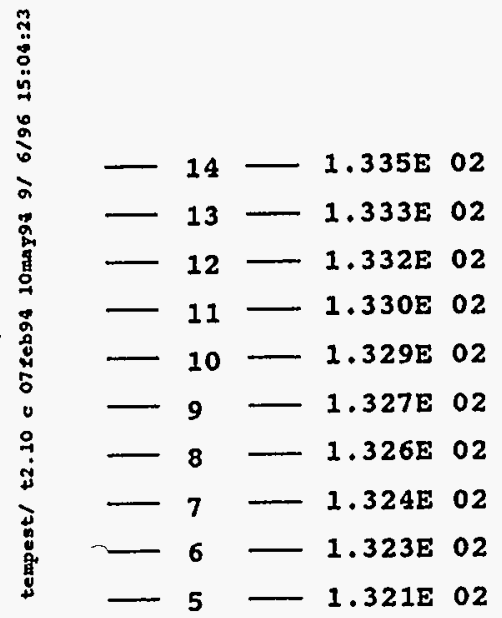

Figure C.29. Predicted Horizontal Distribution of Solid 2 Volume Fraction on the Tank Bottom at Two Simulation Hours for Case 1 

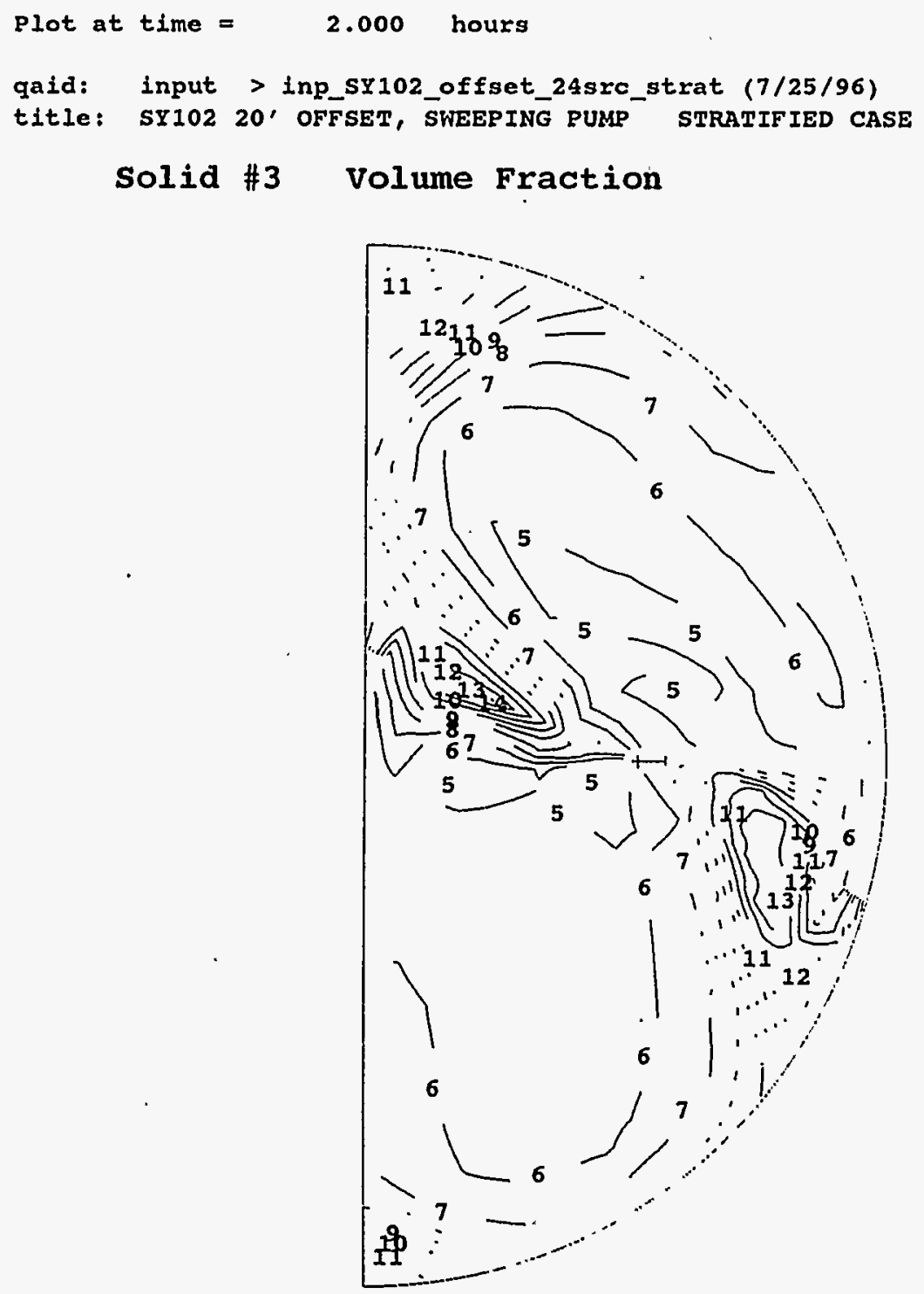
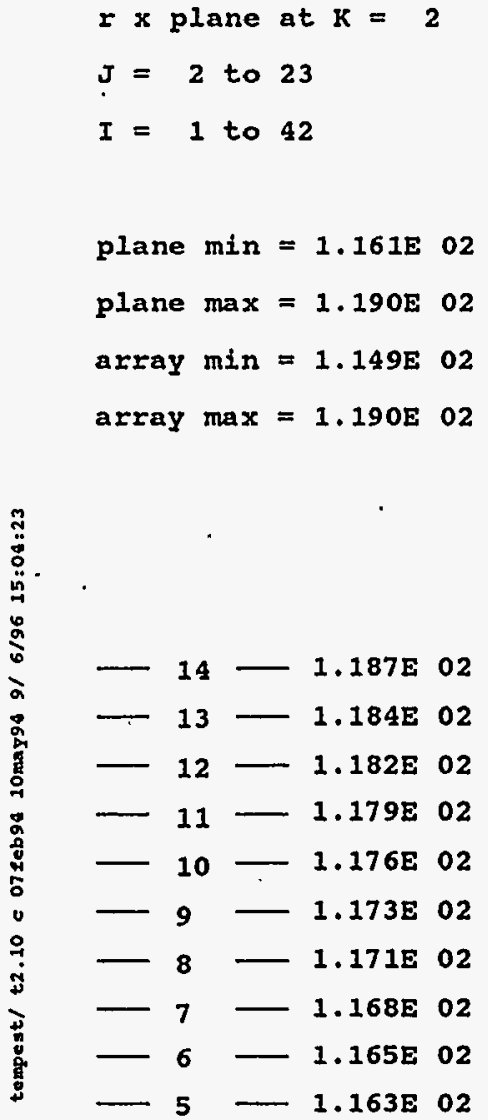

Figure C.30. Predicted Horizontal Distribution of Solid 3 Volume Fraction on the Tank Bottom at Two Simulation Hours for Case 1 
Plot at time $=\quad 2.000$ hours

qaid:" input > inp_sY102_offset_24src_strat $(7 / 25 / 96)$

title: SY102 20' OFFSET, SWEEPING PUMP STRATIFIED CASE

Solid \#4. Volume Fraction

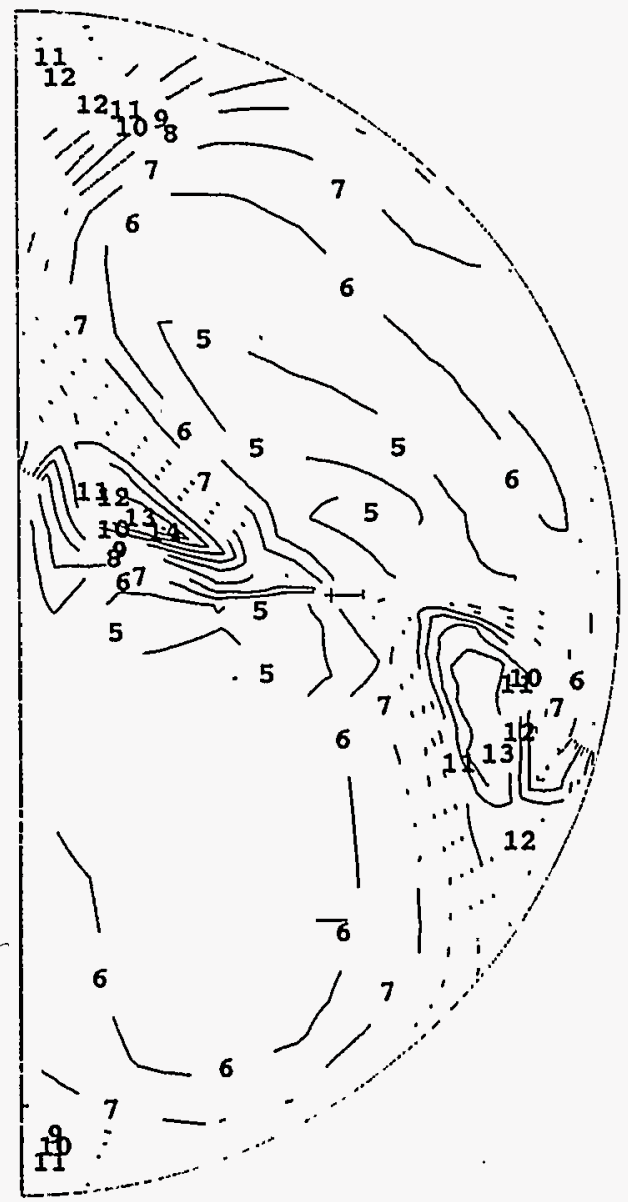

$x$ plane at $K=2$

$J=2$ to 23

$I=1$ to 42

plane $\min =2.802 \mathrm{E} 02$

plane $\max =2.916 \mathrm{E} 02$

array $\min =2.754 \mathrm{E} 02$

array max $=2.916 \mathrm{E} 02$

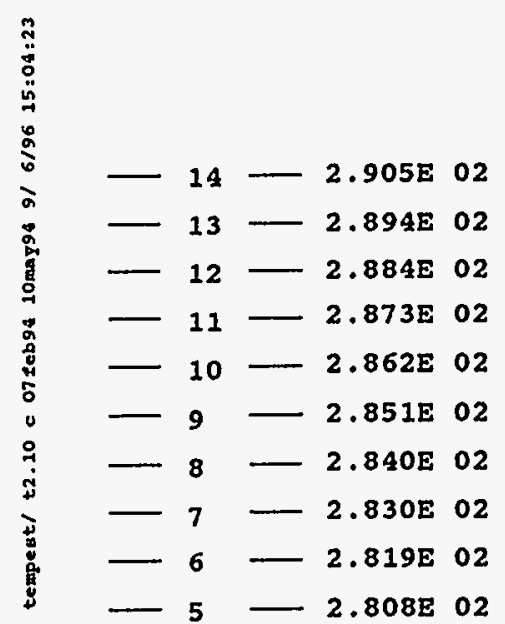

Figure C.31. Predicted Horizontal Distribution of Solid 4 Volume Fraction on the Tank Bottom at Two Simulation Hours for Case 1 
qaid: input > inp_sy102_offset_24src_strat $(7 / 25 / 96)$

title: SY102 $20^{\prime}$ OFFSET, SWEEPING PUMP STRATIFIED CASE

Solid \#5

Volume Fraction

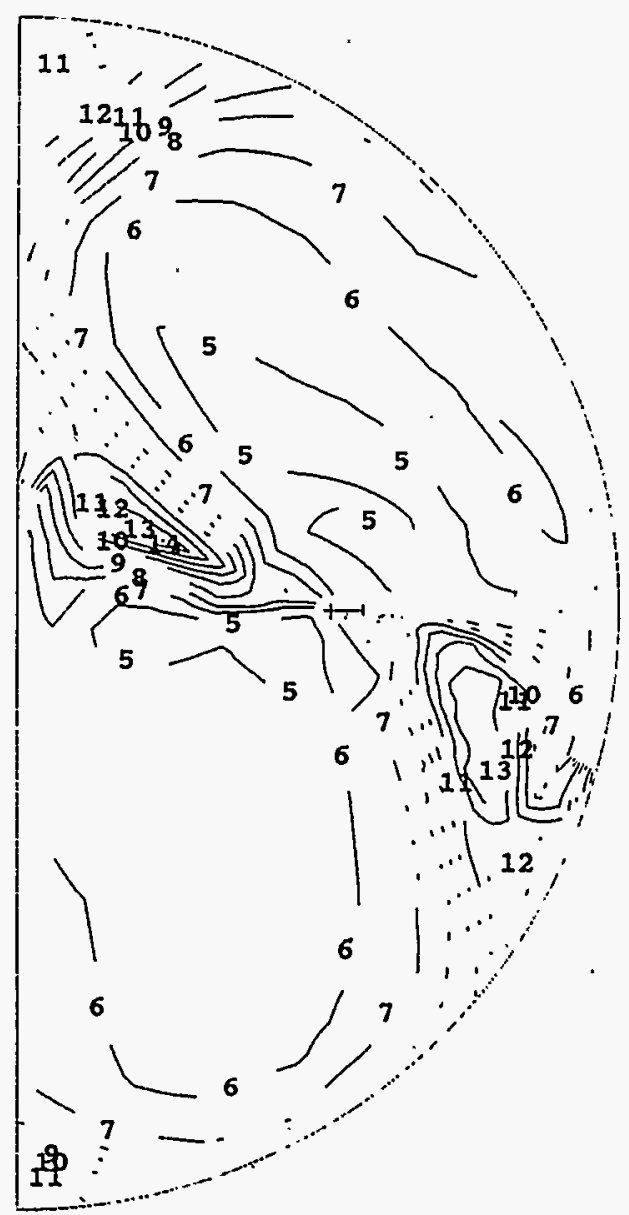

I $x$ plane at $k=2$

$J=2$ to 23

$I=1$ to 42

plane $\min =5.019 \mathrm{E} 02$ plane $\max =5.322 \mathrm{E} 02$ array $\mathrm{min}=4.893 \mathrm{E} 02$ array $\max =5.322 \mathrm{E} \quad 02$

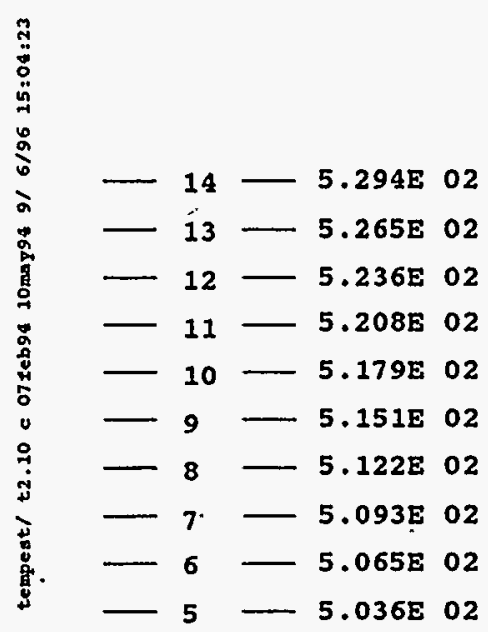

Figure C.32. Predicted Horizontal Distribution of Solid 5 Volume Fraction on the Tank Bottom at Two Simulation Hours for Case 1 


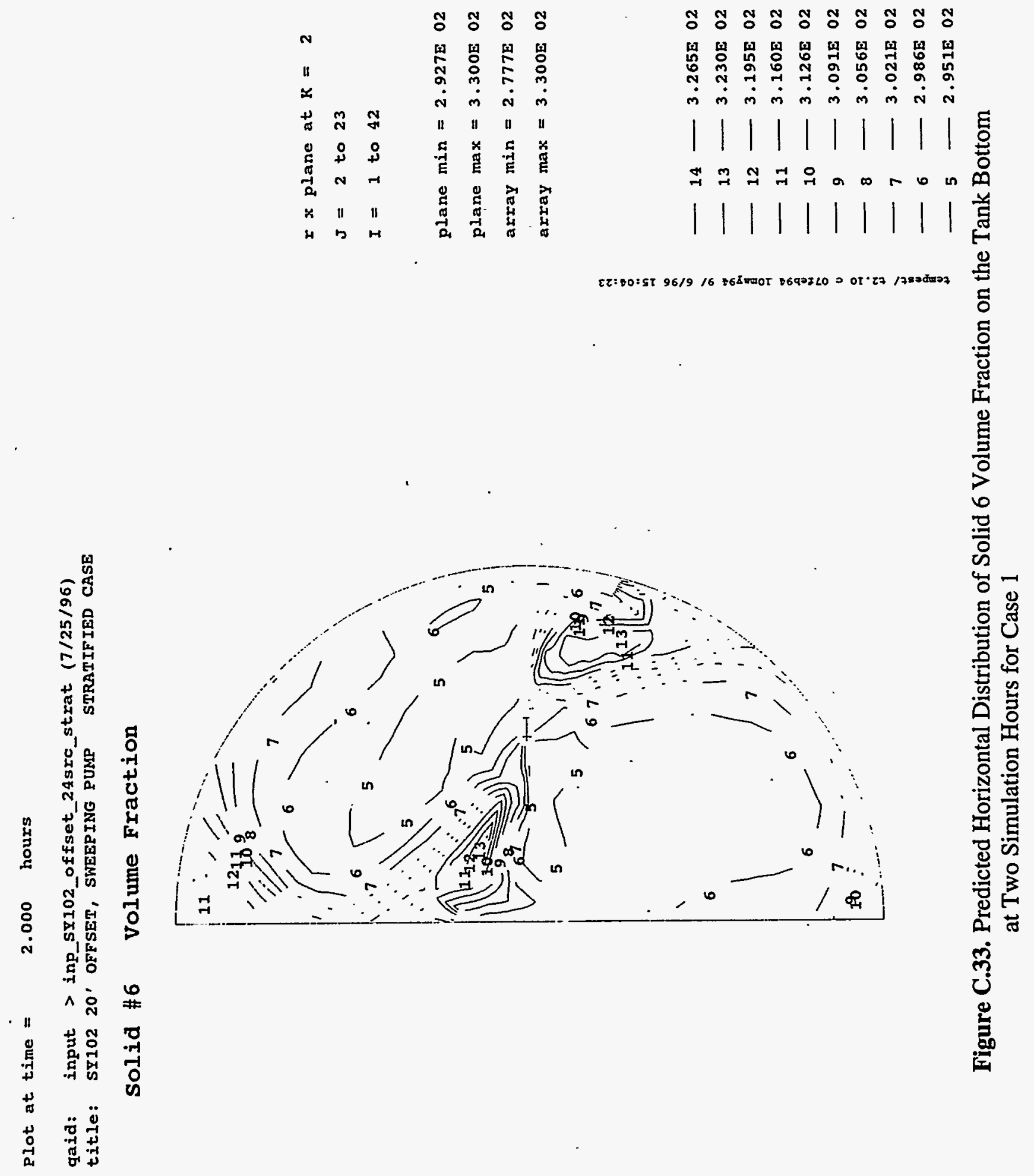




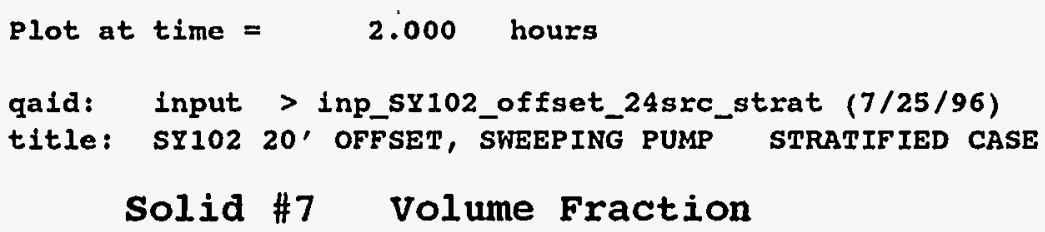

\section{Solid \#7 Volume Fraction}

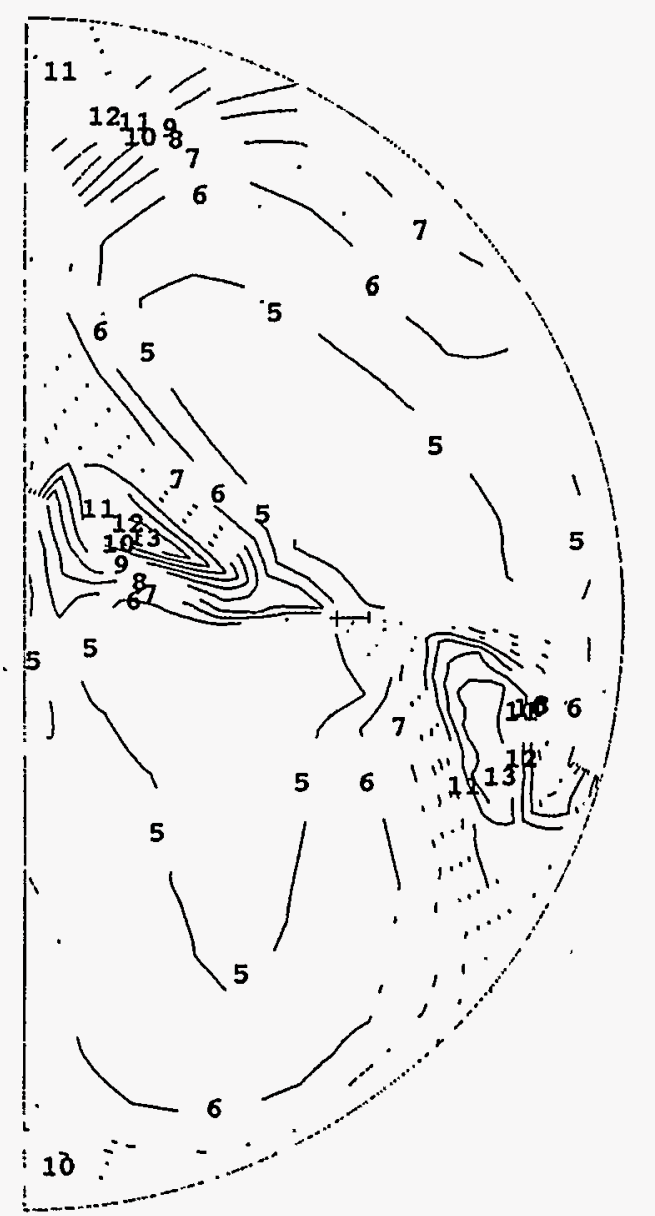

$x$ plane at $k=2$

$J=2$ to 23

$I=1$ to 42

plane $\min =6.364 \mathrm{E} 03$ plane $\max =8.741 \mathrm{E} 03$ array $\min =5.480 \mathrm{E} 03$ array $\max =8.741 \mathrm{E} 03$

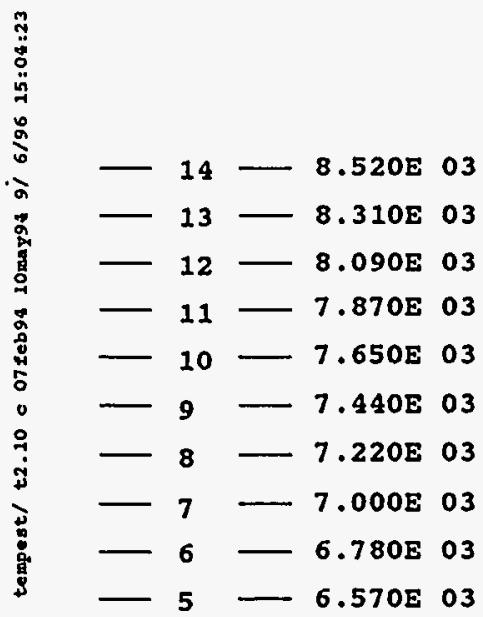

Figure C.34. Predicted Horizontal Distribution of Solid 7 Volume Fraction on the Tank Bottom at Two Simulation Hours for Case 1 


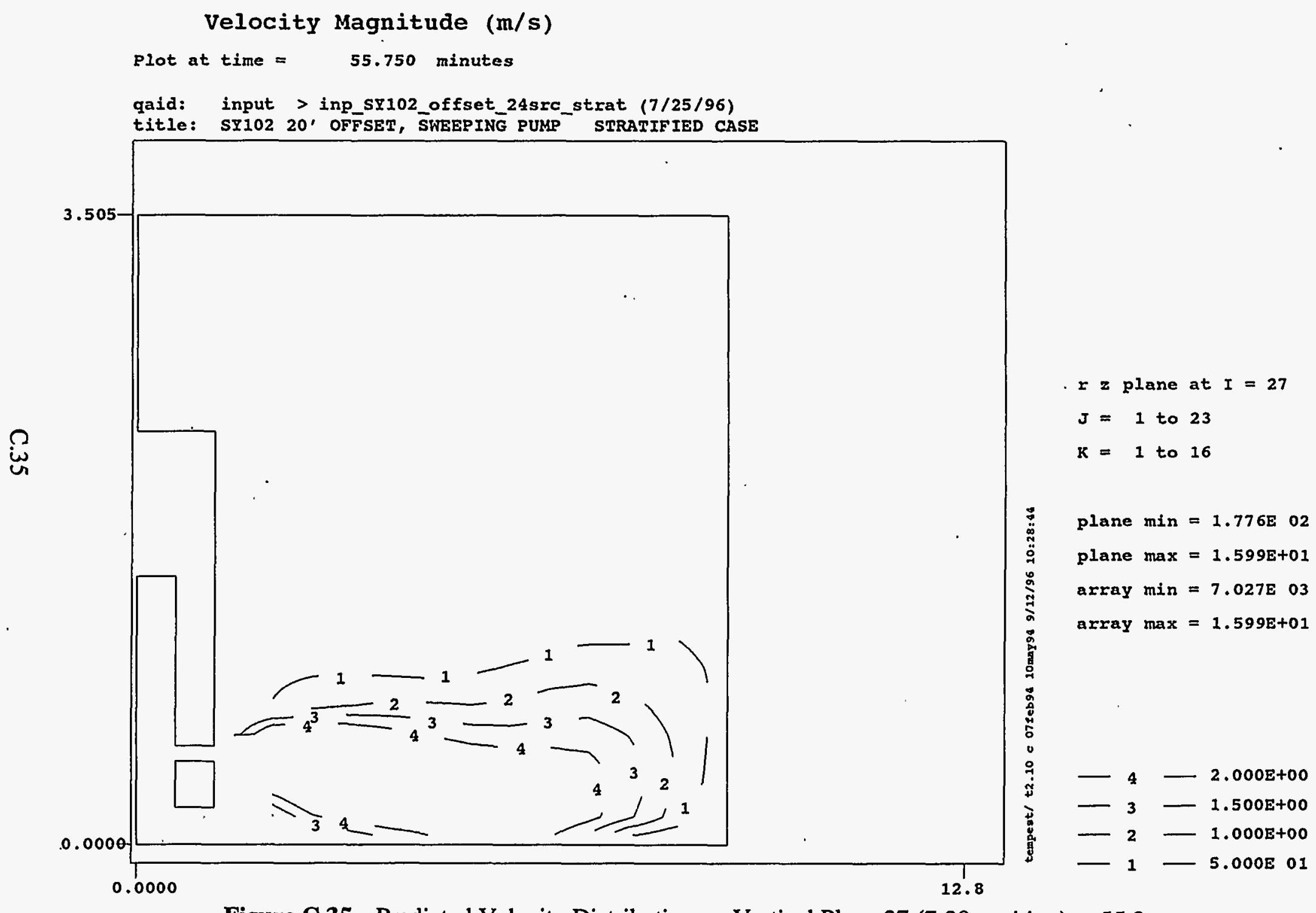

Figure C.35. Predicted Velocity Distribution on Vertical Plane 27 (7:30 position) at 55.8

Simulation Minutes for Case 1 


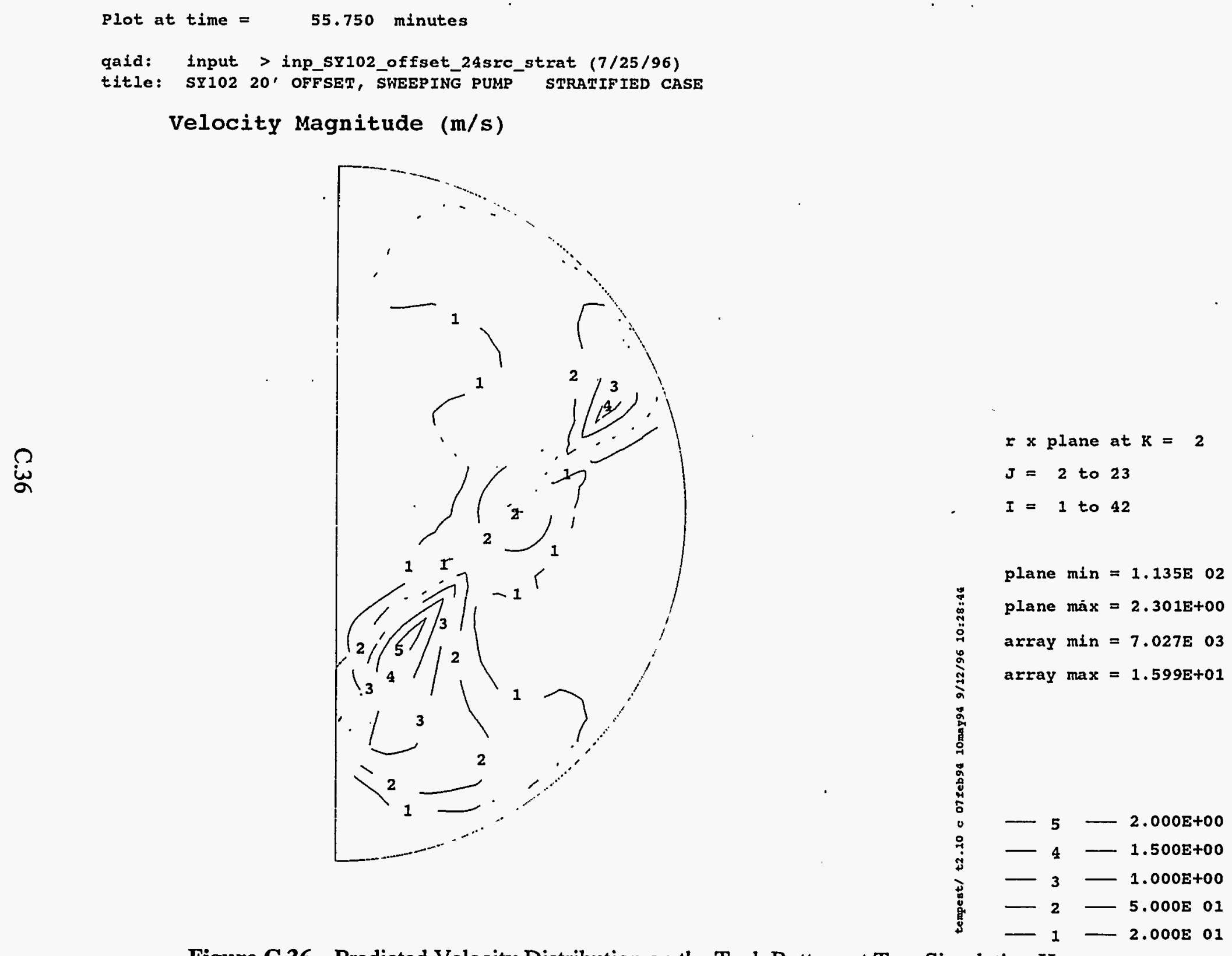

Figure C.36. Predicted Velocity Distribution on the Tank Bottom at Two Simulation Hours at 55.8 Simulation Minutes for Case 1 
$0.2 \mathrm{~m} / \mathrm{s}, 26 \mathrm{~Pa}$

$\mathrm{r}=10.5 \mathrm{~m}(34 \mathrm{ft})$

$0.5 \mathrm{~m} / \mathrm{s}, 160 \mathrm{~Pa}$

$r=10 \mathrm{~m}(32 \mathrm{ft})$

$1.0 \mathrm{~m} / \mathrm{s}, 650 \mathrm{~Pa}$

$\mathrm{r}=9 \mathrm{~m}(29 \mathrm{ft})$

$2.0 \mathrm{~m} / \mathrm{s}, 2600 \mathrm{~Pa}$

$\mathrm{r}=7 \mathrm{~m}(22 \mathrm{ft})$

$\stackrel{\Omega}{\omega}$

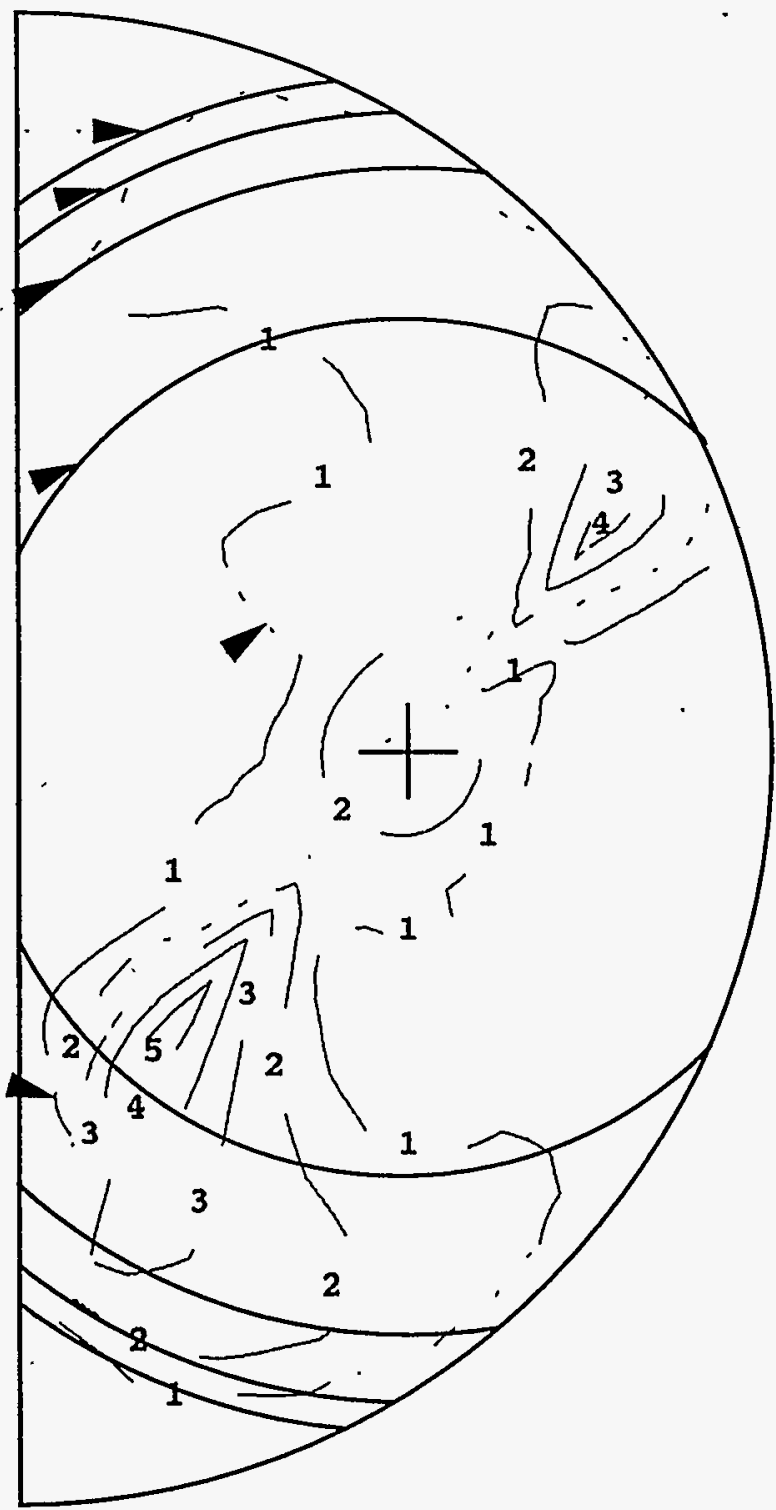

Figure C.37. Estimated Tank Bottom Areas for Possible Sludge Mobilization at Different Velocity and Yield Stress Values for Case 1 


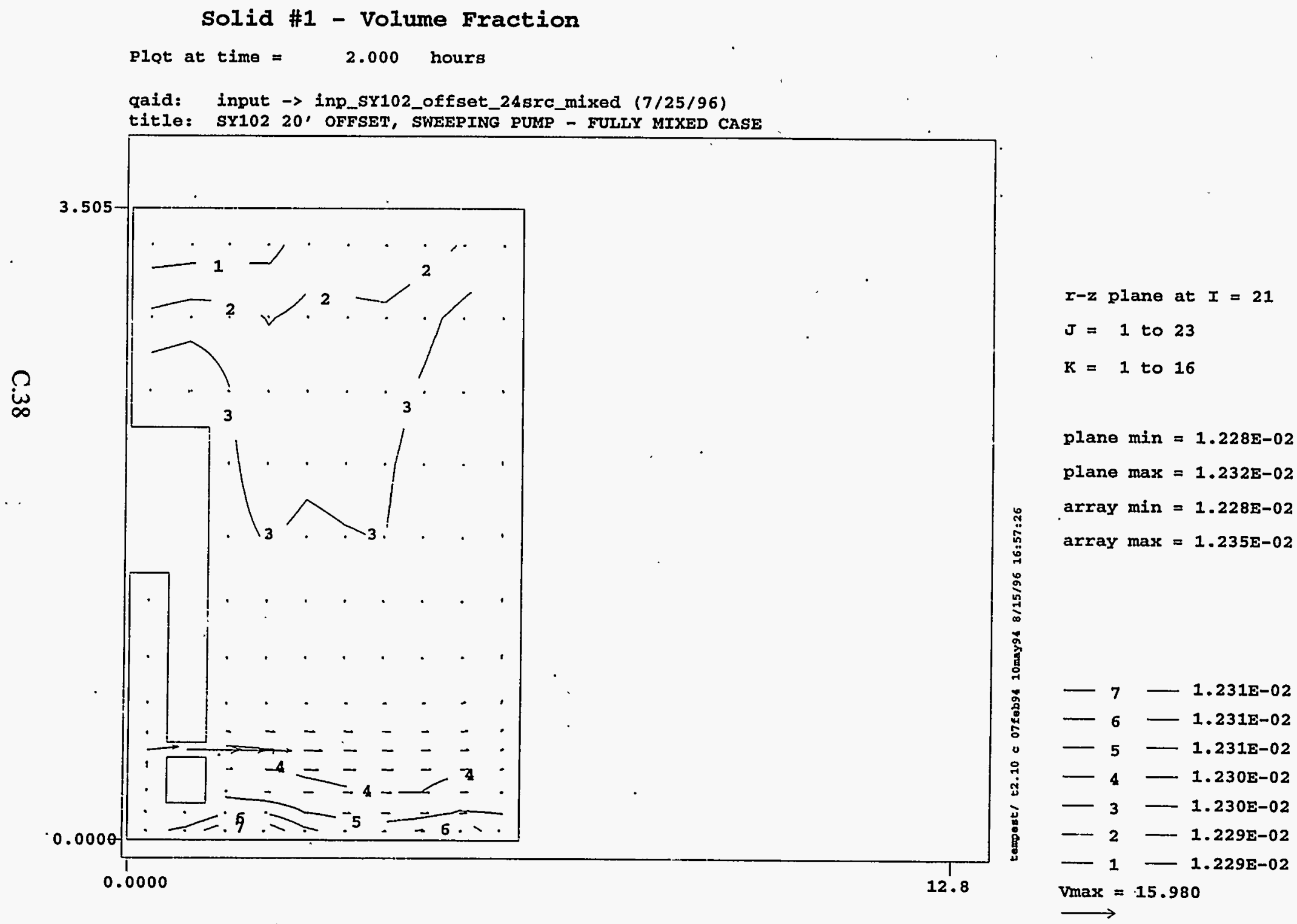

Figure C.38. Predicted Velocity $(\mathrm{m} / \mathrm{s})$ and Distribution of Solid 1 Volume Fraction on Vertical Plane 21 (9 o'clock position) at Two Simulation Hours for Case 2 


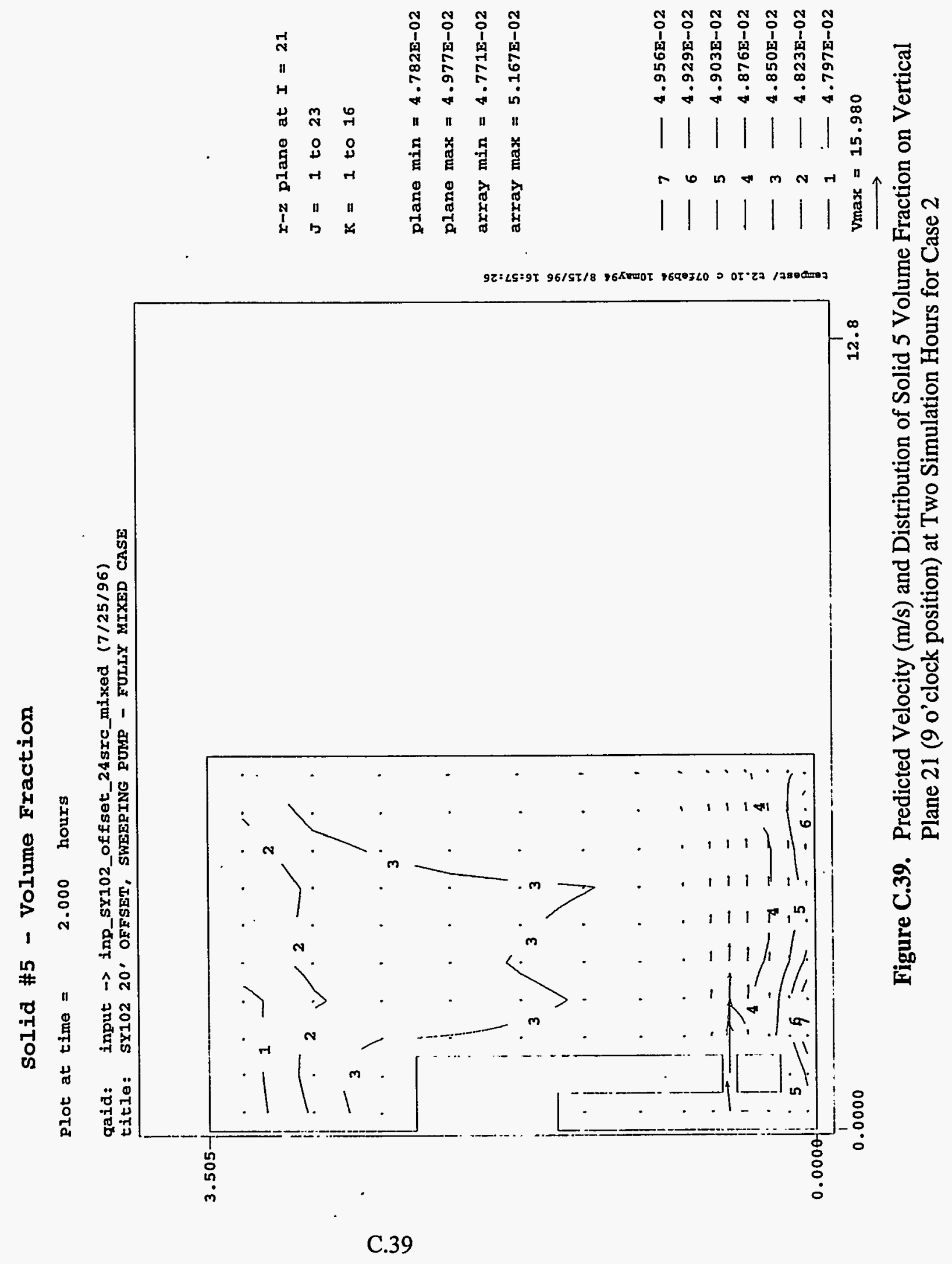




\section{Solid \#7 - Volume Fraction}

\section{Plot at time $=2.000$ hours}

qaid: input $\rightarrow$ inp_SY102_offset_24src_mixed $(7 / 25 / 96)$

tit1e: SY102 20' OFFSET, SWEEPING PUMP - FULLY MIXED CASE

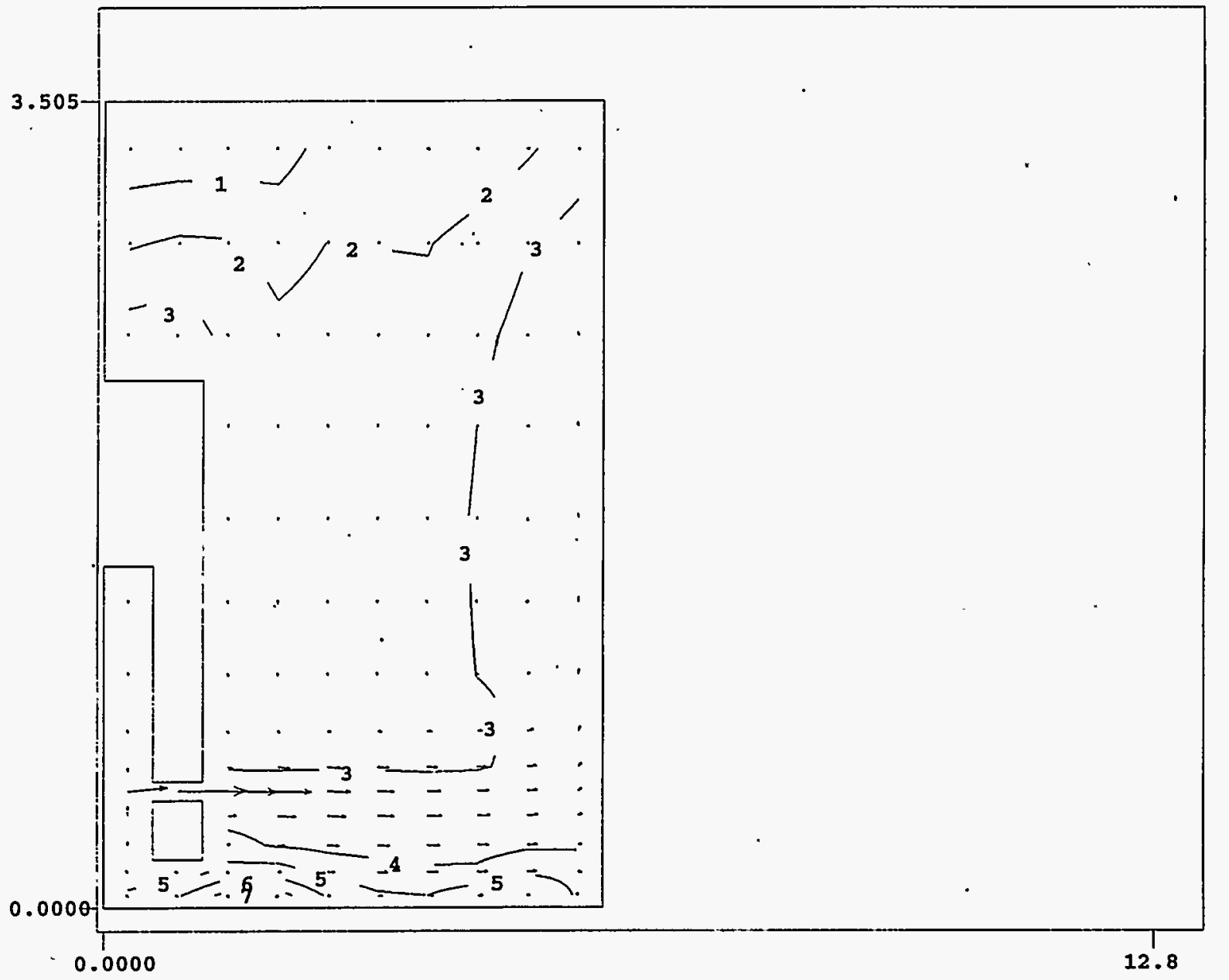

$x-z$ plane at $I=21$

$J=1$ to 23

$\kappa=1$ to 16

plane $\min =6.012 \mathrm{E}-03$

plane $\max =7.591 \mathrm{E}-03$

array $\min =5.931 \mathrm{E}-03$

array $\max =9.258 \mathrm{E}-03$

$-7-7.480 \mathrm{E}-03$

$-6-7.260 \mathrm{E}-03$

- $5-7.040 \mathrm{E}-03$

$-4-6.820 \mathrm{E}-03$

- $3 \longrightarrow 6.600 \mathrm{E}-03$

$\therefore 2-6.370 \mathrm{E}-03$

$-1-6.150 \mathrm{E}-03$

$v_{\max }=15.980$

Figure C.40. Predicted Velocity $(\mathrm{m} / \mathrm{s})$ and Distribution of Solid 7 Volume Fraction on Vertical Plane 21 (9 o'clock position) at Two Simulation Hours for Case 2 
qaid: input > inp_sY102_offset_24src_mixed $(7 / 25 / 96)$

title: SY102 20' OFFSET, SWEEPING PUMP FULLY MIXED CASE

\section{Solid \#1 Volume Fraction}

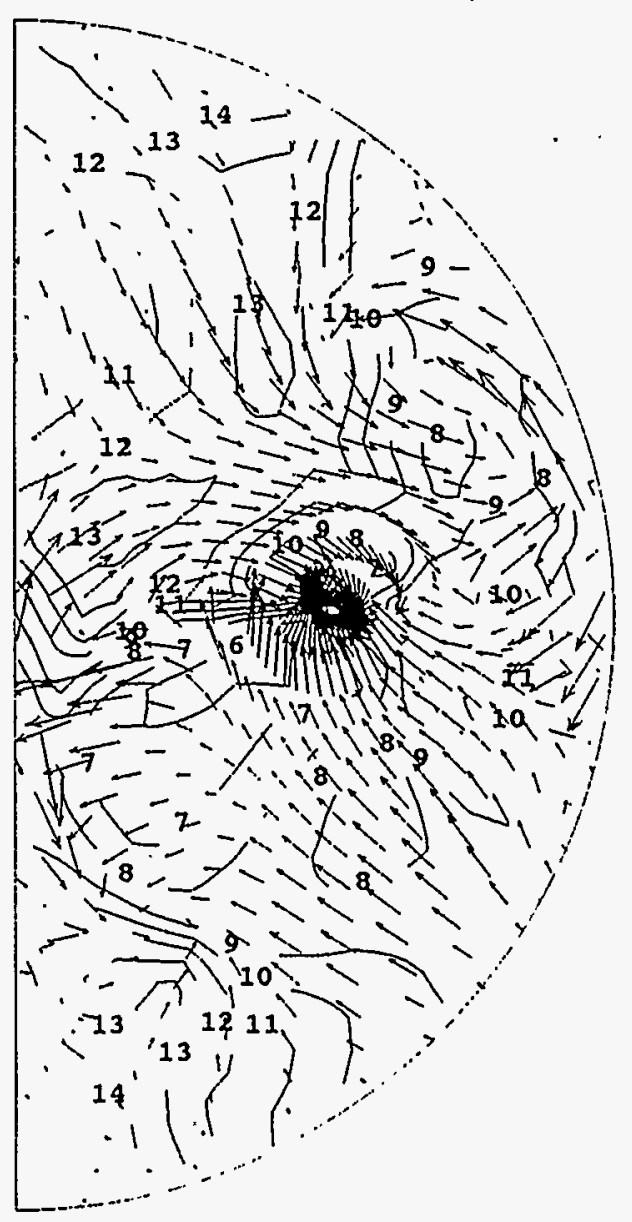

I $x$ plane at $k=2$

$J=2$ to 23

$I=1$ to 42

plane $\min =1.230 \mathrm{E} 02$

plane $\max =1.240 \mathrm{E} 02$

array $\min =1.225 \mathrm{E} 02$

array $\max =1.240 \mathrm{E} 02$$$
\text { : }
$$

$-14-1.239 \mathrm{E} 02$

$-13-1.238 \mathrm{E} 02$

$-12-1.237 \mathrm{E} 02$

$-11-1.236 \mathrm{E} 02$

- $10-1.235 \mathrm{E} 02$

- $9-1.234 \mathrm{E} 02$

- $8-1.233 \mathrm{E} 02$

$-7-1.232802$

$-6-1.231 \mathrm{E} 02$

$V \max =0.424$

Figure C.41. Predicted Velocity $(\mathrm{m} / \mathrm{s})$ and Horizontal Distribution of Solid 1 Volume Fraction on the Tank Bottom at Two Simulation Hours for Case 2 
qaid: input > inp_SY102_offset_24src_mixed $(7 / 25 / 96)$

title: SI102 20' OFFSET, SWEEPING PUMP FULLY MIXED CASE

\section{Solid \#5 Volume Fraction}

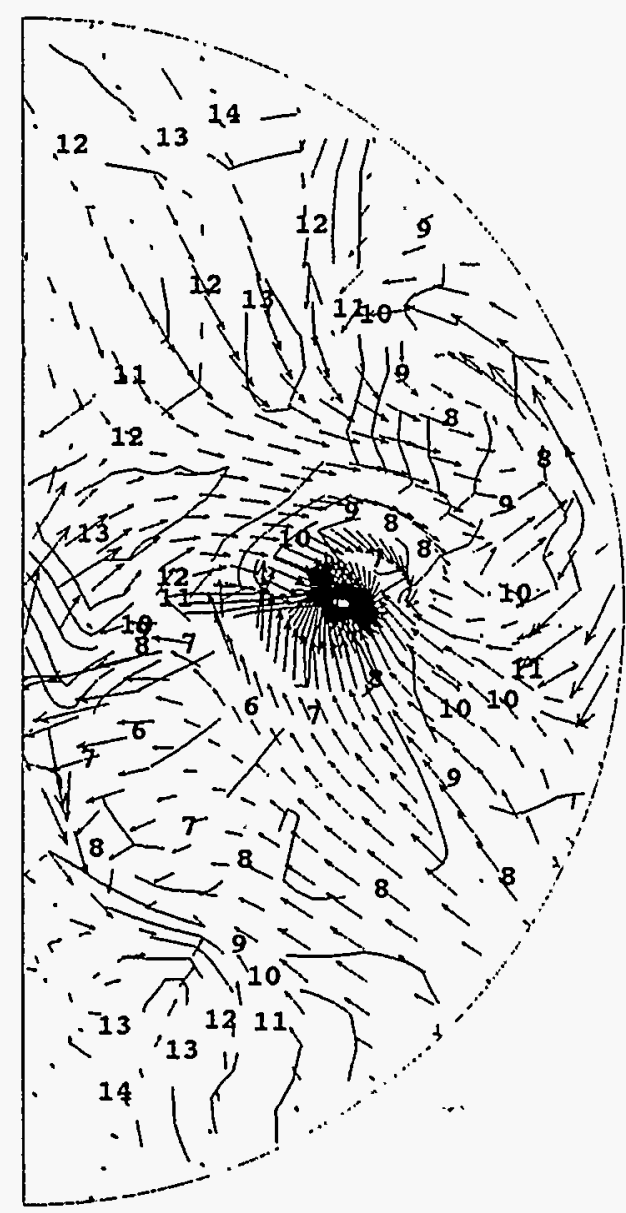

$x$ plane at $k=2$

$J=2$ to 23

$I=1$ to 42

Plane $\min =4.895 \mathrm{E} 02$

plane $\max =5.454 \mathrm{E} 02$

array $\min =4.592 \mathrm{E} 02$

array $\max =5.454 \mathrm{E} 02$$$
\text { . }
$$

$-14-5.397 \mathrm{E} 02$

$-13-5.339 \mathrm{E} 02$

$-12-5.282 \mathrm{E} 02$

$-11-5.224 E 02$

$-10-5.167 \mathrm{E} 02$

- $-5.109 \mathrm{E} 02$

- $8-5.052 \mathrm{E} 02$

$-7-4.994 \mathrm{E} 02$

- $6-4.937 \mathrm{E} 02$

$v_{\max }=0.424$

Figure C.42. Predicted Velocity $(\mathrm{m} / \mathrm{s})$ and Horizontal Distribution of Solid 5 Volume Fraction on the Tank Bottom at Two Simulation Hours for Case 2 
qaid: input $>$ inp_SY102_offset_24src_mixed $(7 / 25 / 96)$

title: SY102 20' OFFSET, SWEEPING PUMP FULLY MIXED CASE

\section{Solid \#7 Volume Fraction}

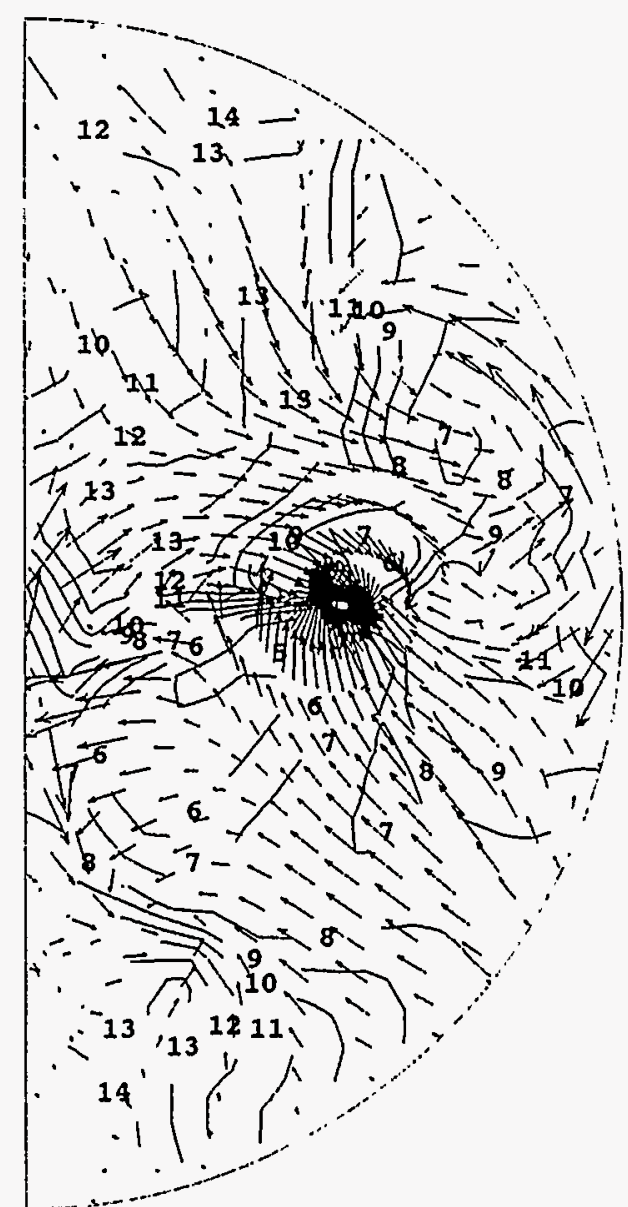

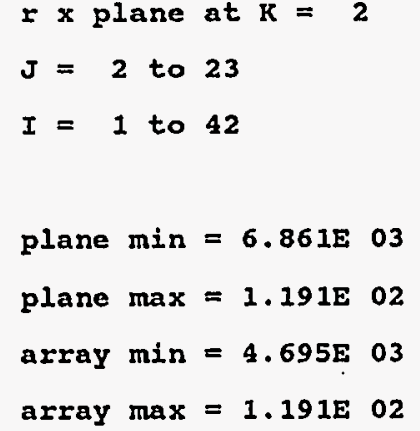

- $14-1.143 \mathrm{E} 02$

$-13-1.095 \mathrm{E} 02$

- $12-1.047 \mathrm{E} 02$

$-11-9.990 \mathrm{E} \mathrm{03}$

- $10-9.510 \mathrm{E} 03$

- $9-9.030 \mathrm{E} 03$

- $8-8.550 \mathrm{E} 03$

- $7-8.060 \mathrm{E} 03$

$-6-7.580 \mathrm{E} 03$

$-5-7.100 \mathrm{E} 03$

$v_{\max }=0.424$

Figure C.43. Predicted Velocity $(\mathrm{m} / \mathrm{s})$ and Horizontal Distribution of Solid 7 Volume Fraction on the Tank Bottom at Two Simulation Hours for Case 2 


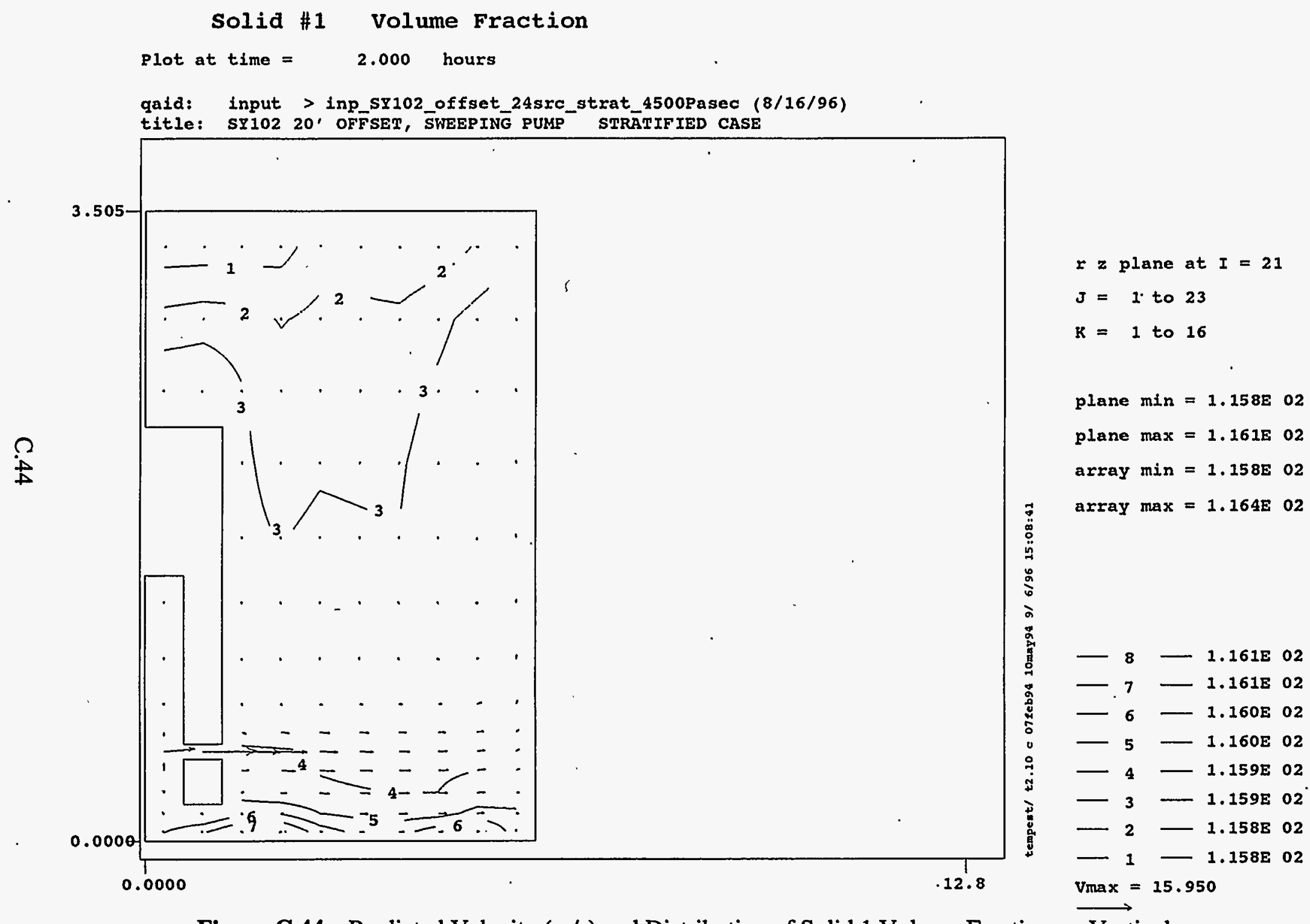

Figure C.44. Predicted Velocity $(\mathrm{m} / \mathrm{s})$ and Distribution of Solid 1 Volume Fraction on Vertical Plane 21 (9 o'clock position) at Two Simulation Hours for Case 3 
qaid: input > inp_SY102_offset_24src_strat_4500Pasec $(8 / 16 / 96)$

title: SY102 20' OFFSET, SWEEPING PUMP STRATIFIED CASE

\section{Solid \#1 Volume Fraction}

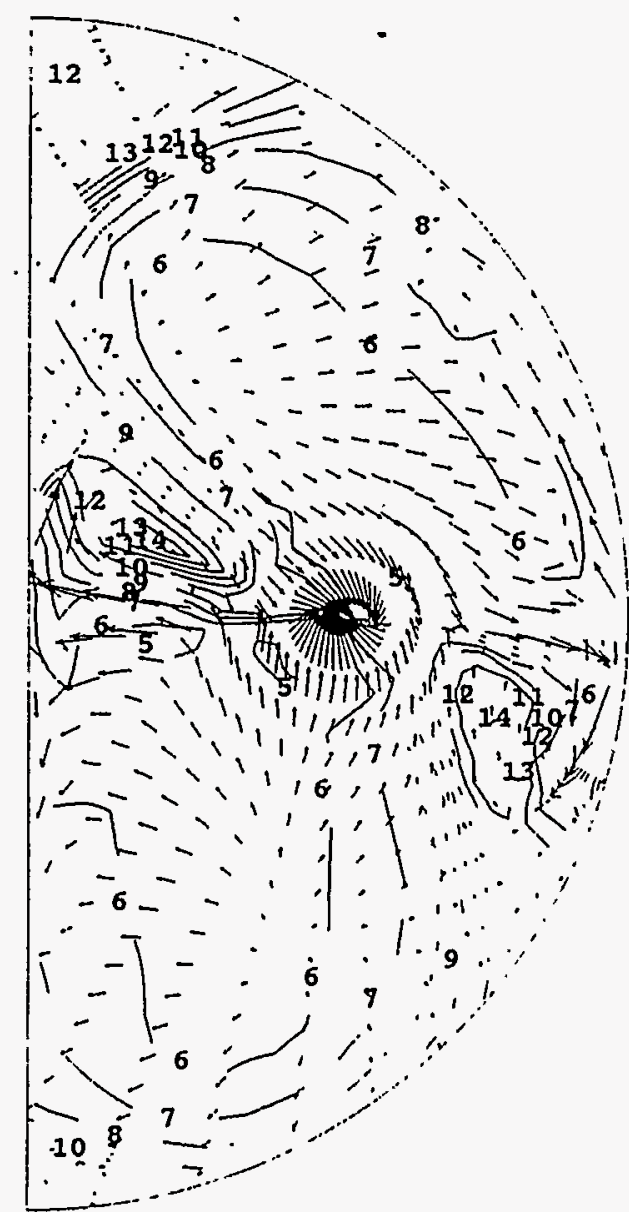

$\mathrm{r} \times$ plane at $\mathrm{K}=2$

$J=2$ to 23

$I=1$ to 42

plane $\min =1.160 \mathrm{E} 02$

plane $\max =1.164 \mathrm{E} 02$

array $\min =1.158 \mathrm{E} 02$

array $\max =1.164 \mathrm{E} 02$

$-14-1.164 \mathrm{E} 02$

- $13-1.163 \mathrm{E} 02$

- $12-1.163 \mathrm{E} 02$

$-11-1.162 \mathrm{E} 02$

$-10-1.162 \mathrm{E} 02$

$-9-1.161 \mathrm{E} 02$

$-8-1.161 E 02$

- $7-1.161 \mathrm{E} 02$

- $6-1.160 \mathrm{E} 02$

5. $1.160 \mathrm{E} 02$

$V \max =1.626$

Figure C.45. Predicted Velocity $(\mathrm{m} / \mathrm{s})$ and Horizontal Distribution of Solid 1 Volume Fraction on the Tank Bottom at Two Simulation Hours for Case 3 


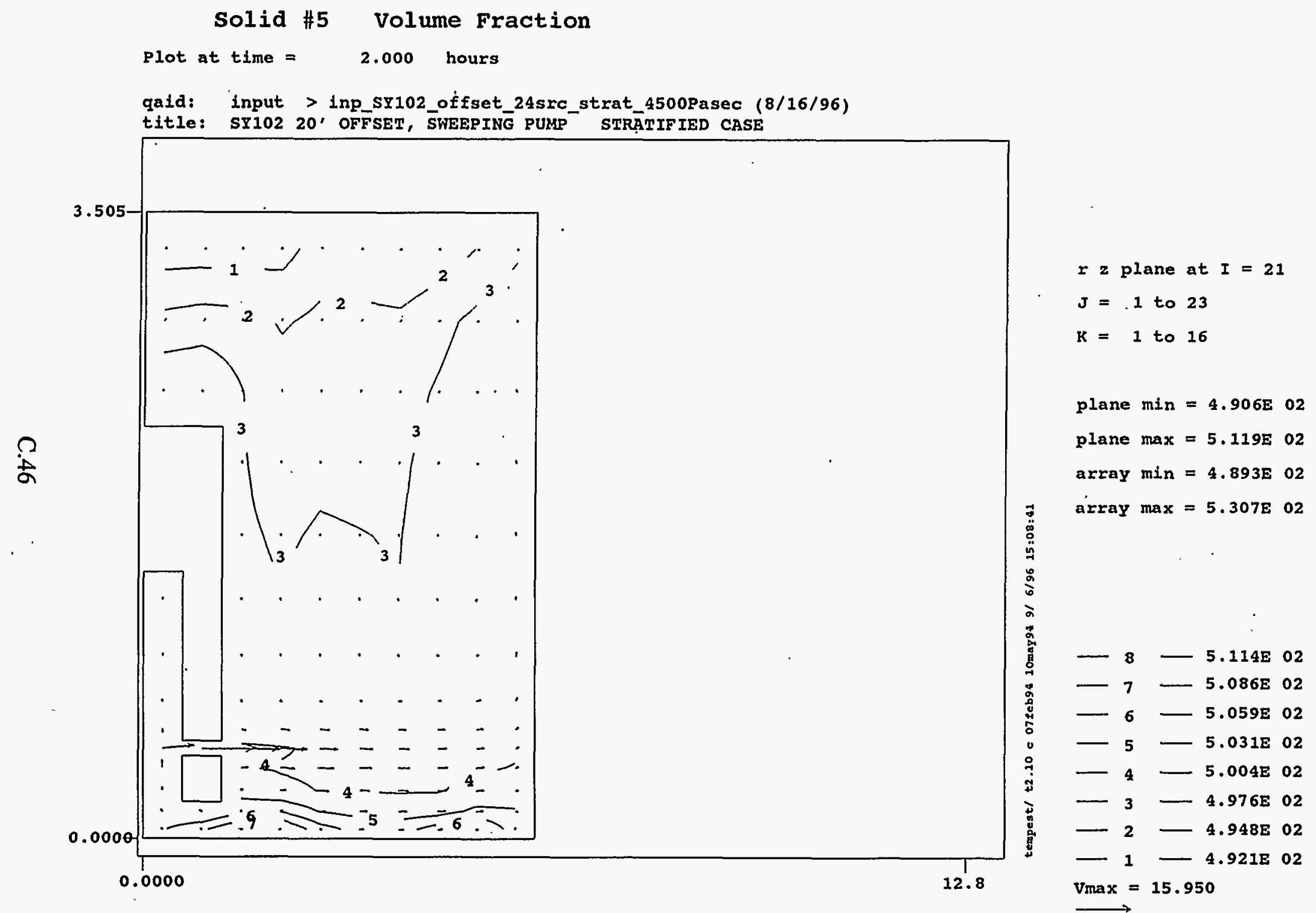

Figure C.46. Predicted Velocity $(\mathrm{m} / \mathrm{s})$ and Distribution of Solid 5 Volume Fraction on Vertical Plane 21 (9:00 Clock Position) at Two Simulation Hours for Case 3 
Plot at time $=\quad 2.000$ hours

qaid: input > inp_SY102_offset_24src_strat_4500Pasec $(8 / 16 / 96)$

title: SY102 20' OFFSET, SWEEPING PUMP STRATIFIED CASE

\section{Solid \#5 Volume Fraction}

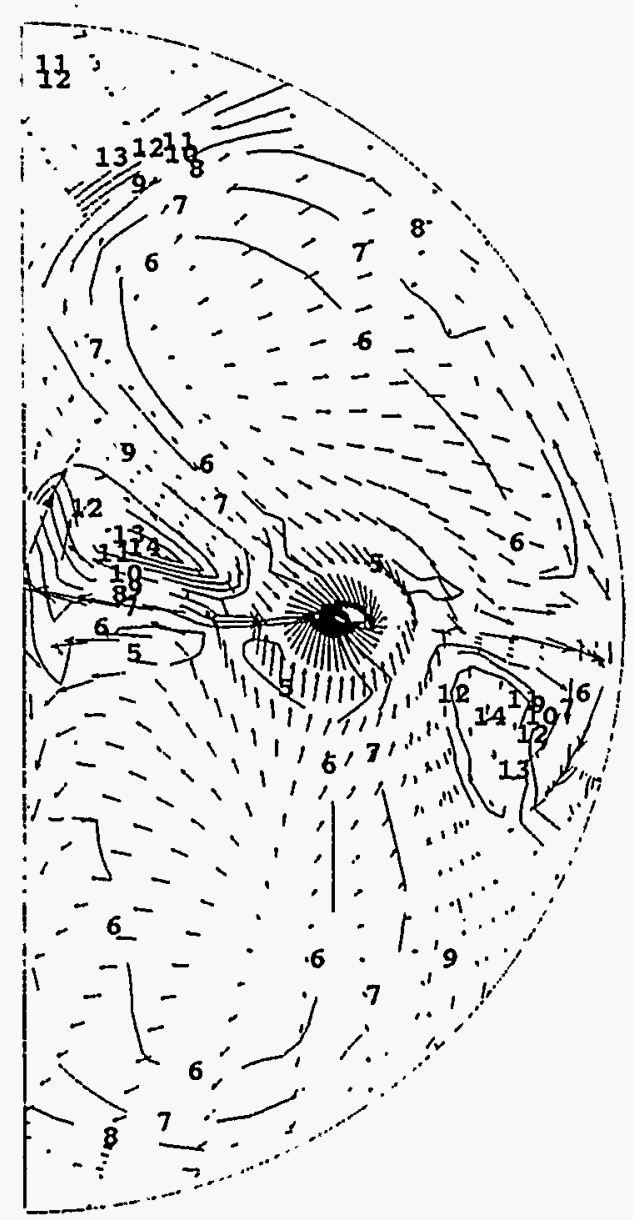

r $x$ plane at $k=2$

$J=2$ to 23

$I=1$ to 42

plane $\min =5.019 \mathrm{E} 02$

plane $\max =5.307 \mathrm{E} 02$

array min $=4.893 \mathrm{E} 02$

array $\max =5.307 \mathrm{E} 02$

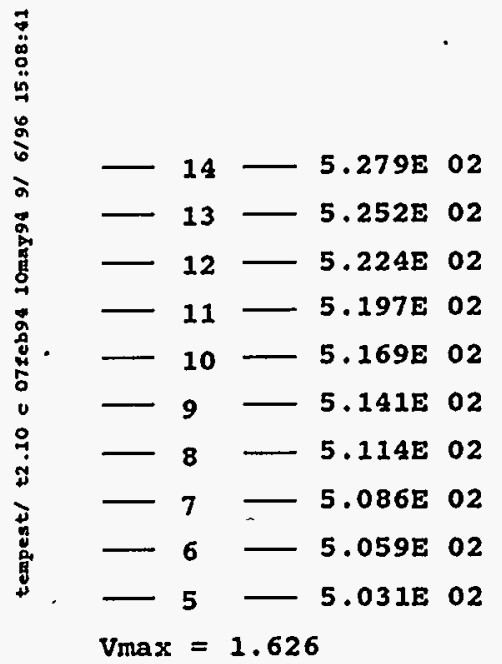

Figure C.47. Predicted Velocity $(\mathrm{m} / \mathrm{s})$ and Horizontal Distribution of Solid 5 Volume Fraction on the Tank Bottom at two Simulation Hours for Case 3 


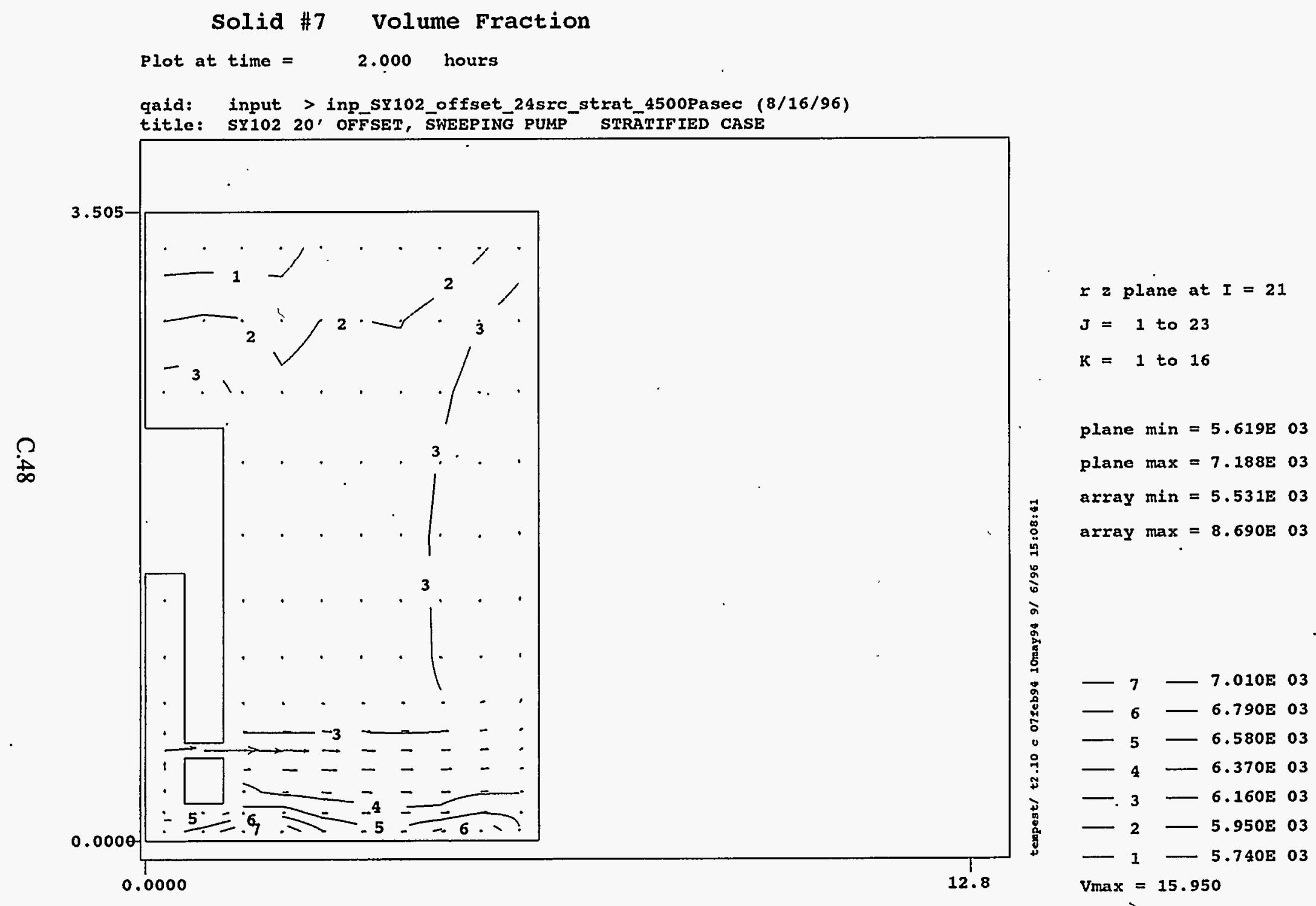

Figure C.48. Predicted Velocity $(\mathrm{m} / \mathrm{s})$ and Distribution of Solid 7 Volume Fraction on Vertical Plane 21 (9 o'clock position) at Two Simulation Hours for Case 3 
qaid: input > inp_SY102_offset_24src_strat_4500Pasec $(8 / 16 / 96)$

title: SY102 20' OFFSET, SWEEPING PUMP STRATIFIED CASE

\section{Solid \#7 Volume Fraction}

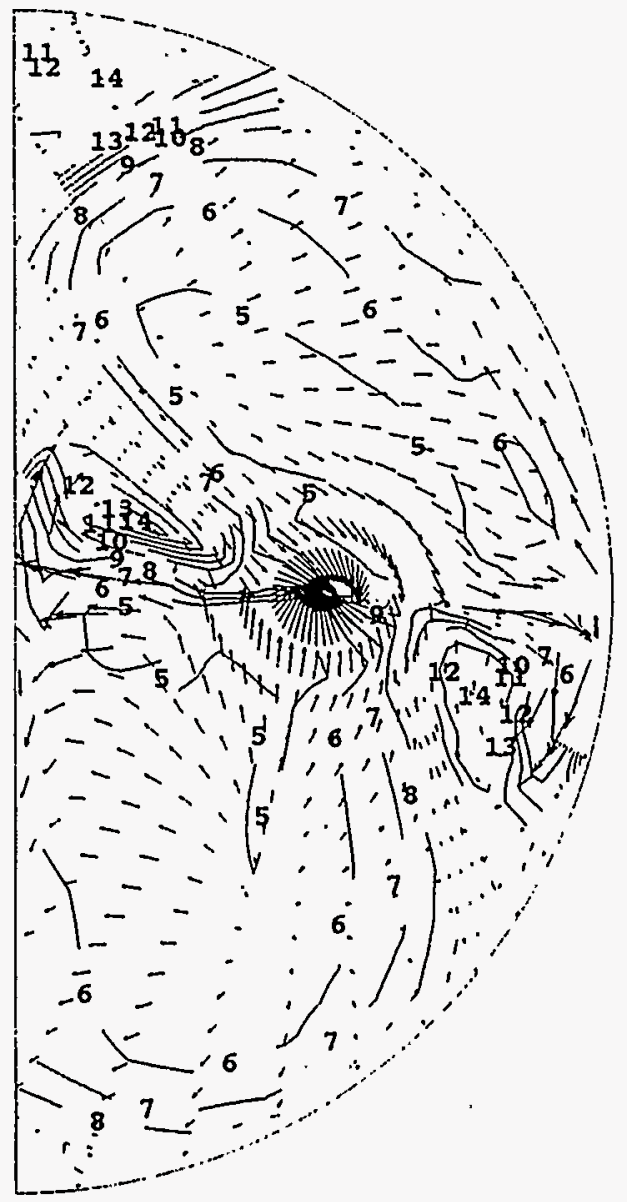

r $x$ plane at $k=2$

$J=2$ to 23

$I=1$ to 42

plane $\min =6.427 \mathrm{E} 03$ plane $\max =8.690 \mathrm{E} 03$ array min $=5.531 \mathrm{E} 03$ array $\max =8.690 \mathrm{E} 03$

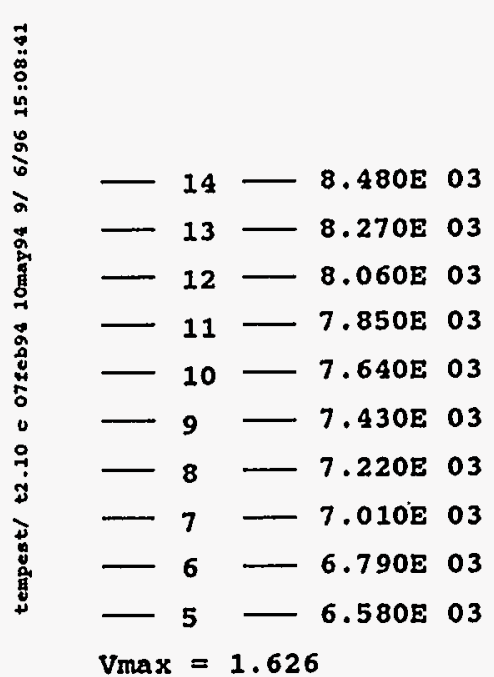

Figure C.49. Predicted Velocity $(\mathrm{m} / \mathrm{s})$ and Horizontal Distribution of Solid 7 Volume Fraction on the Tank Bottom at Two Simulation Hours for Case 3 


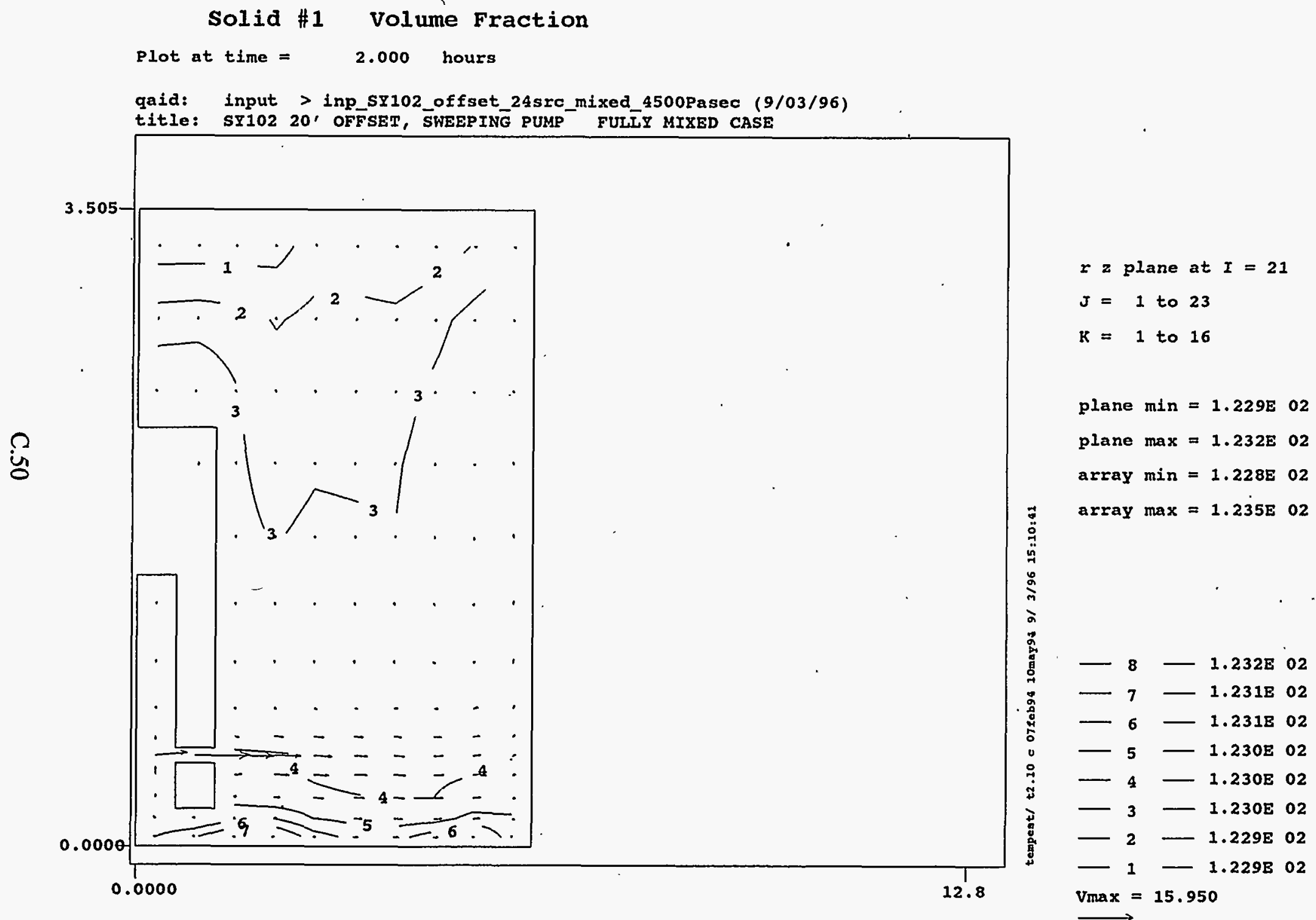

Figure C.50. Predicted Velocity $(\mathrm{m} / \mathrm{s})$ and Distribution of Solid 1 Volume Fraction on Vertical Plane 21 (9 o'clock position) at Two Simulation Hours for Case 4 
qaid: input > inp_sy102_offset_24src_mixed_4500Pasec (9/03/96)

title: SY102 20' OFFSET, SWEEPING PUMP FULII MIXED CASE

\section{Solid \#1 Volume Fraction}

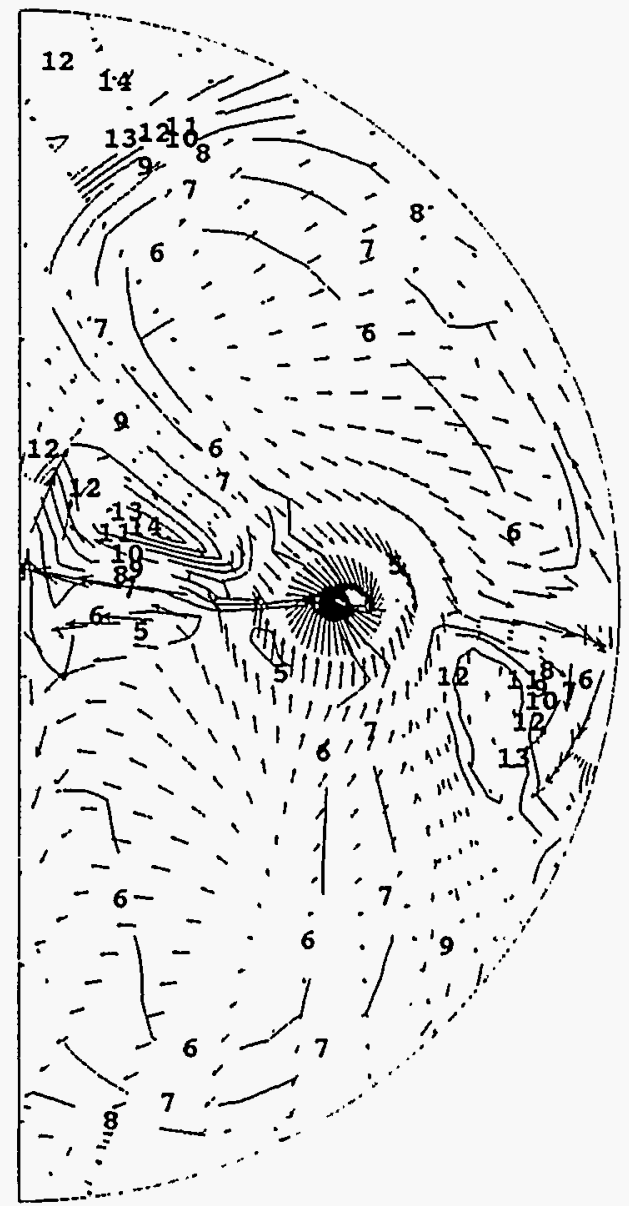

I $x$ plane at $K=2$

$J=2$ to 23

$I=1$ to 42

plane $\min =1.230 \mathrm{E} 02$ plane $\max =1.235 \mathrm{E} 02$ array $\min =1.228 \mathrm{E} 02$ array $\max =1.235 \mathrm{E} 02$

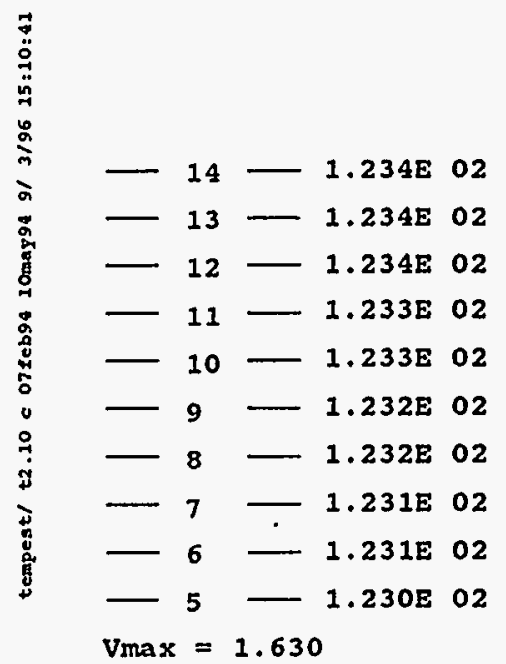

Figure C.51. Predicted Velocity $(\mathrm{m} / \mathrm{s})$ and Horizontal Distribution of Solid 1 Volume Fraction on the Tank Bottom at Two Simulation Hours for Case 4 


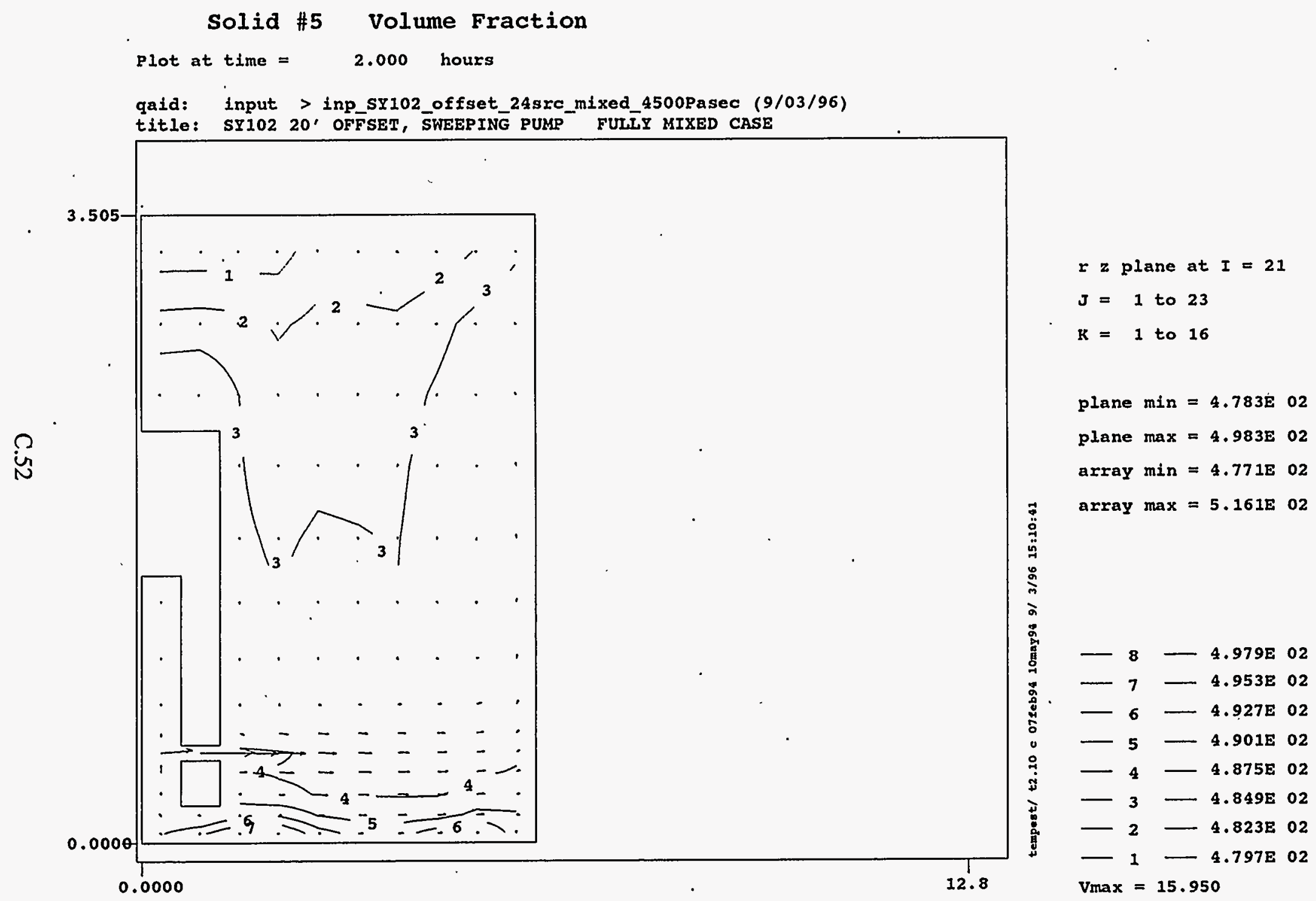

Figure C.52. Predicted Velocity $(\mathrm{m} / \mathrm{s})$ and Distribution of Solid 5 Volume Fraction on Vertical Plane 21 (9 o'clock position) at Two Simulation Hours for Case 4 
qaid: input > inp_SY102_offset_24src_mixed_4500Pasec (9/03/96)

title: SY102 20' OFFSET, SWEEPING PUMP FULIY MIXED CASE

\section{Solid \#5 Volume Fraction}
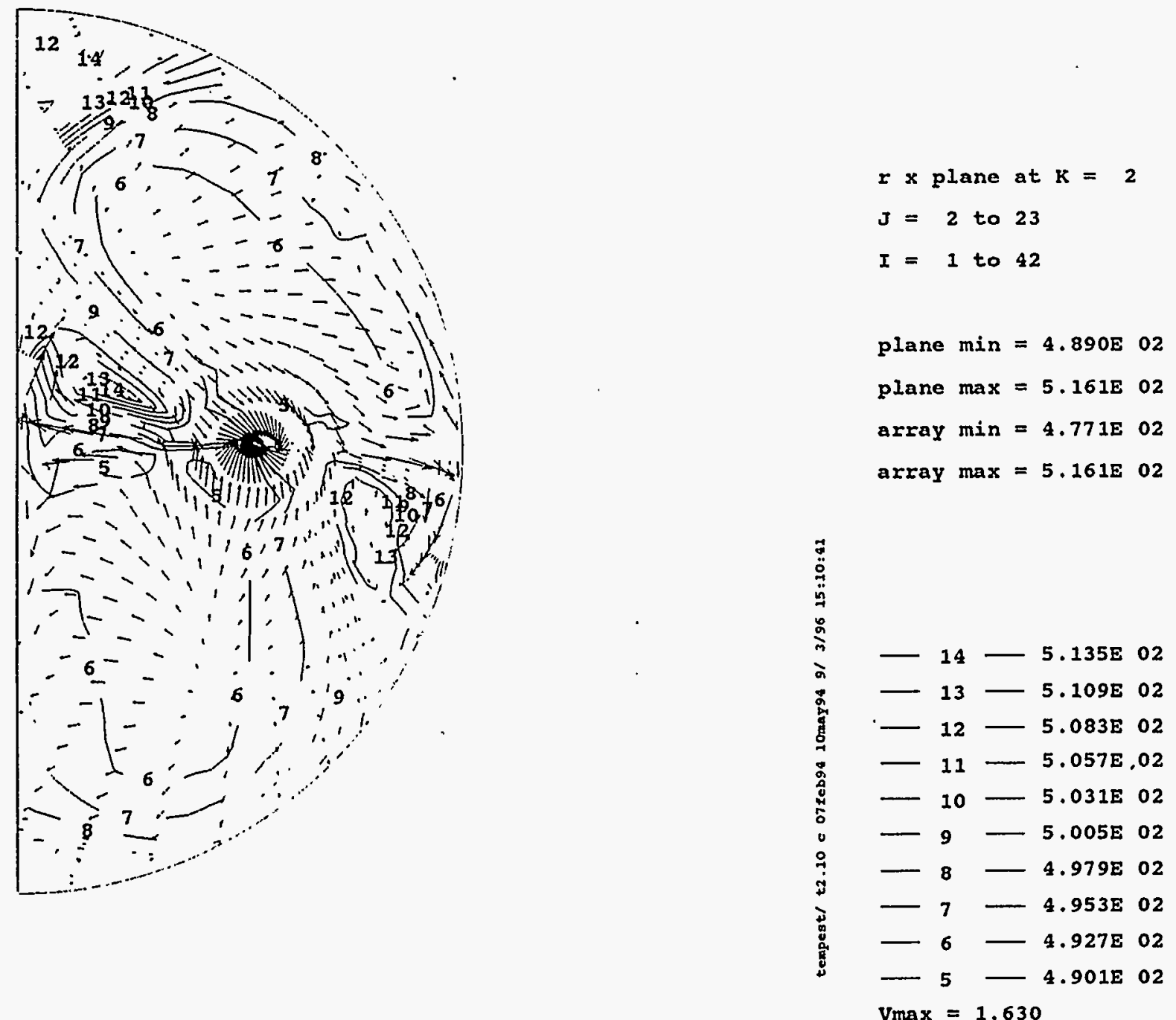

Figure C.53. Predicted Velocity $(\mathrm{m} / \mathrm{s})$ and Horizontal Distribution of Solid 5 Volume Fraction on the Tank Bottom at Two Simulation Hours for Case 4 


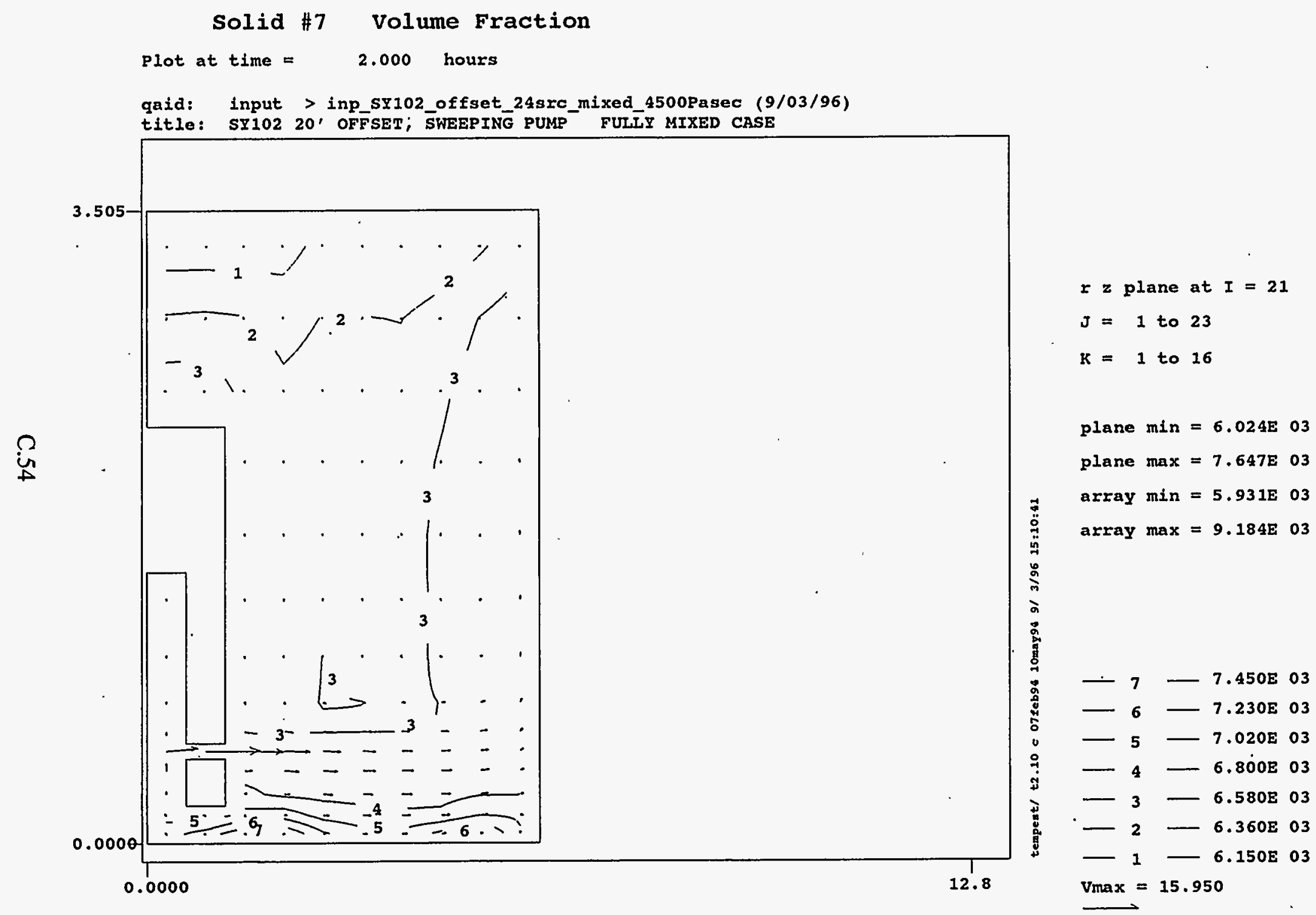

- Figure C.54. Predicted Velocity $(\mathrm{m} / \mathrm{s})$ and Distribution of Solid 7 Volume Fraction on Vertical Plane 21 ( 9 o'clock position) at Two Simulation Hours for Case 4 
qaid: input > inp_sY102_offset_24src_mixed_4500Pasec (9/03/96)

title: SY102 20' OFFSET, SWEEPING PUMP FULLY MIXED CASE

\section{Solid \#7 Volume Fraction}

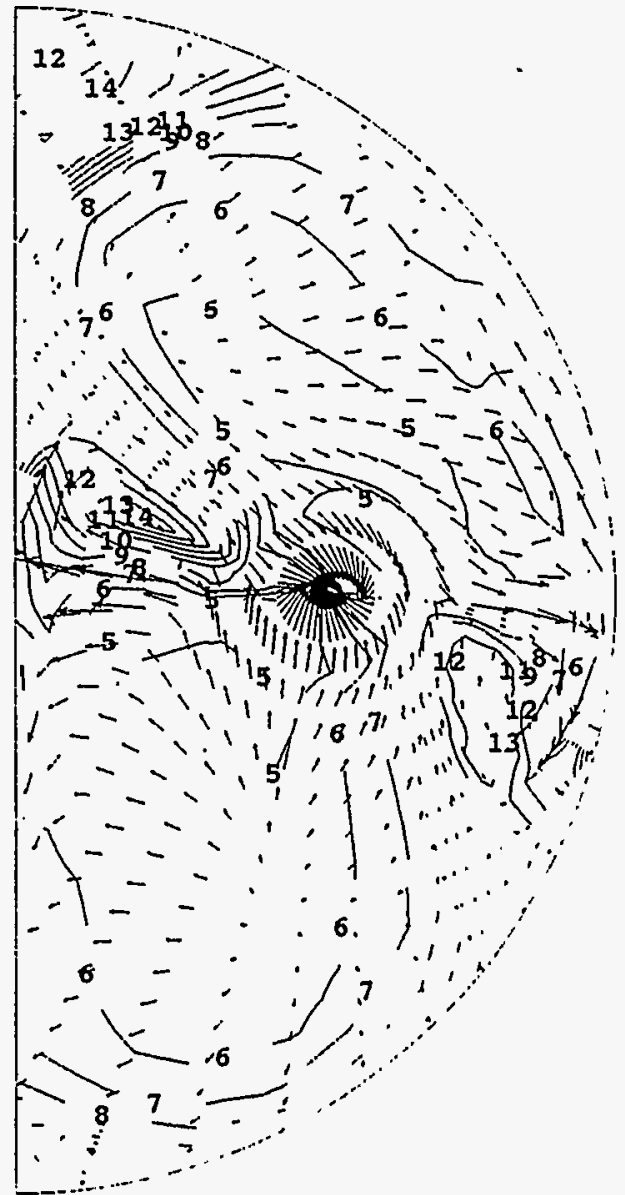

$\mathbf{x}$ plane at $\mathrm{k}=\mathbf{2}$

$J=2$ to 23

$I=1$ to 42

plane $\min =6.860 \mathrm{E} 03$

plane $\max =9.184 \mathrm{E} 03$

array $\min =5.931 \mathrm{E} 03$

array $\max =9.184 \mathrm{E} 03$

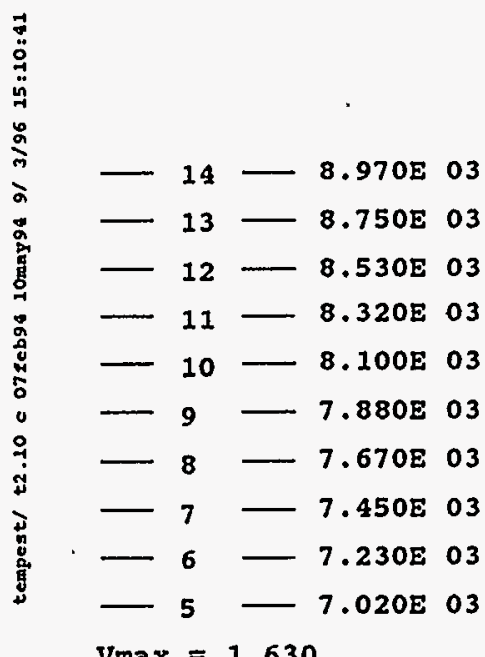

Figure C.55. Predicted Velocity $(\mathrm{m} / \mathrm{s})$ and Horizontal Distribution of Solid 7 Volume Fraction on the Tank Bottom at Two Simulation Hours for Case 4 


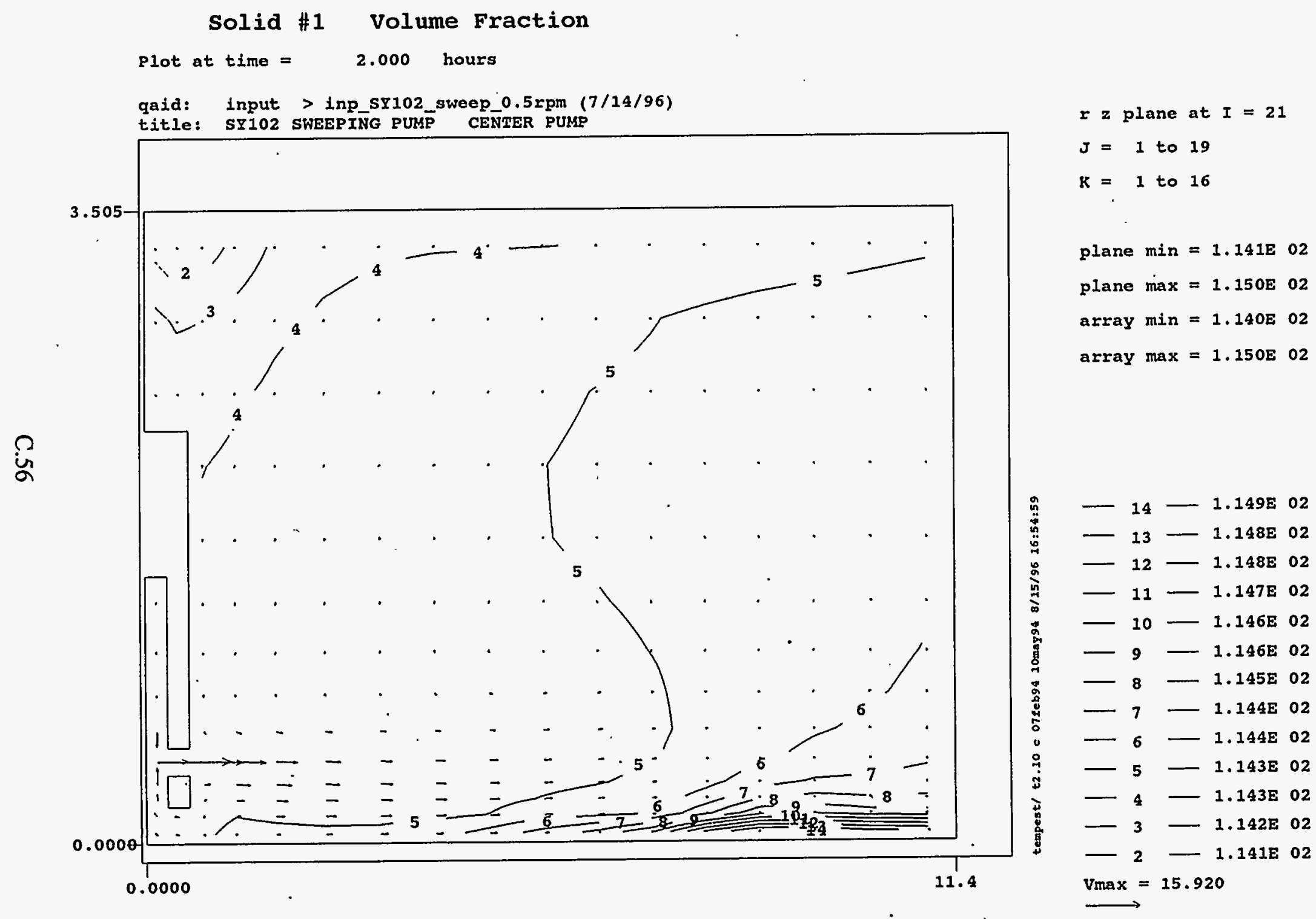

Figure C.56. Predicted Velocity $(\mathrm{m} / \mathrm{s})$ and Distribution of Solid 1 Volume Fraction on Vertical Plane 21 (9 o'clock position) at Two Simulation Hours for Case 5 


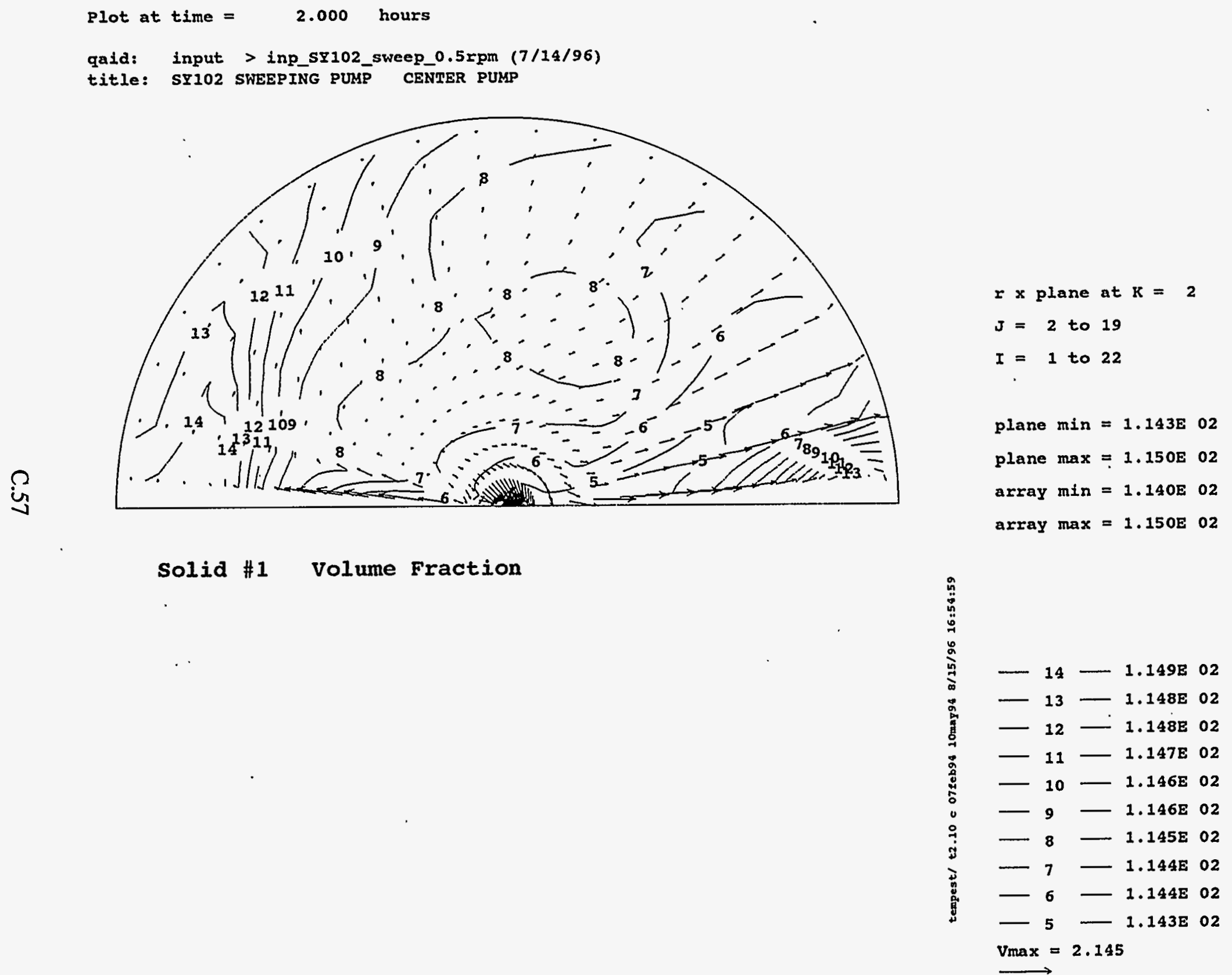

Figure C.57. Predicted Velocity $(\mathrm{m} / \mathrm{s})$ and Horizontal Distribution of Solid 1 Volume Fraction on the Tank Bottom at Two Simulation Hours for Case 5 


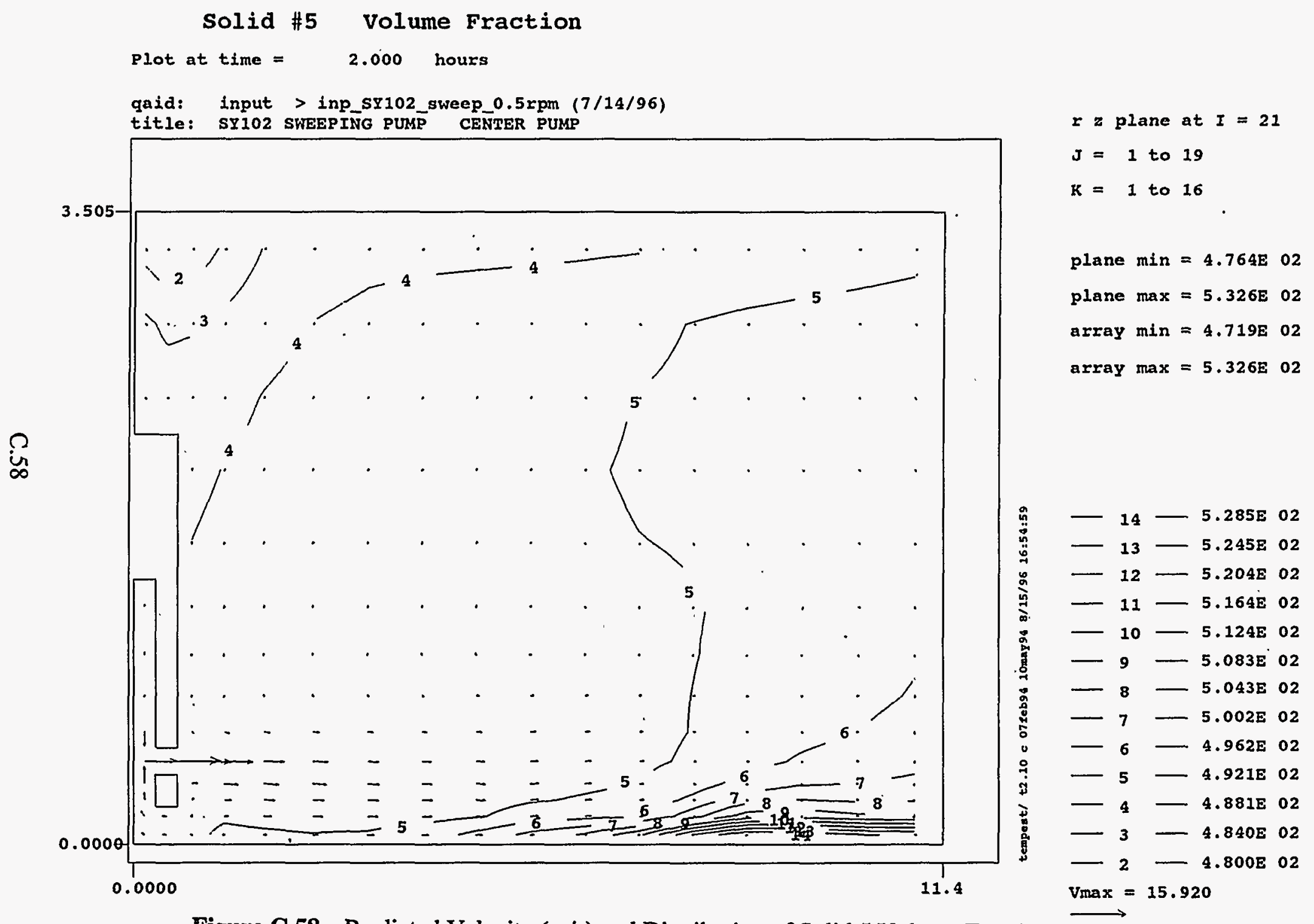

Figure C.58. Predicted Velocity $(\mathrm{m} / \mathrm{s})$ and Distribution of Solid 5 Volume Fraction on Vertical Plane 21 (9 o'clock position) at Two Simulation Hours for Case 5 


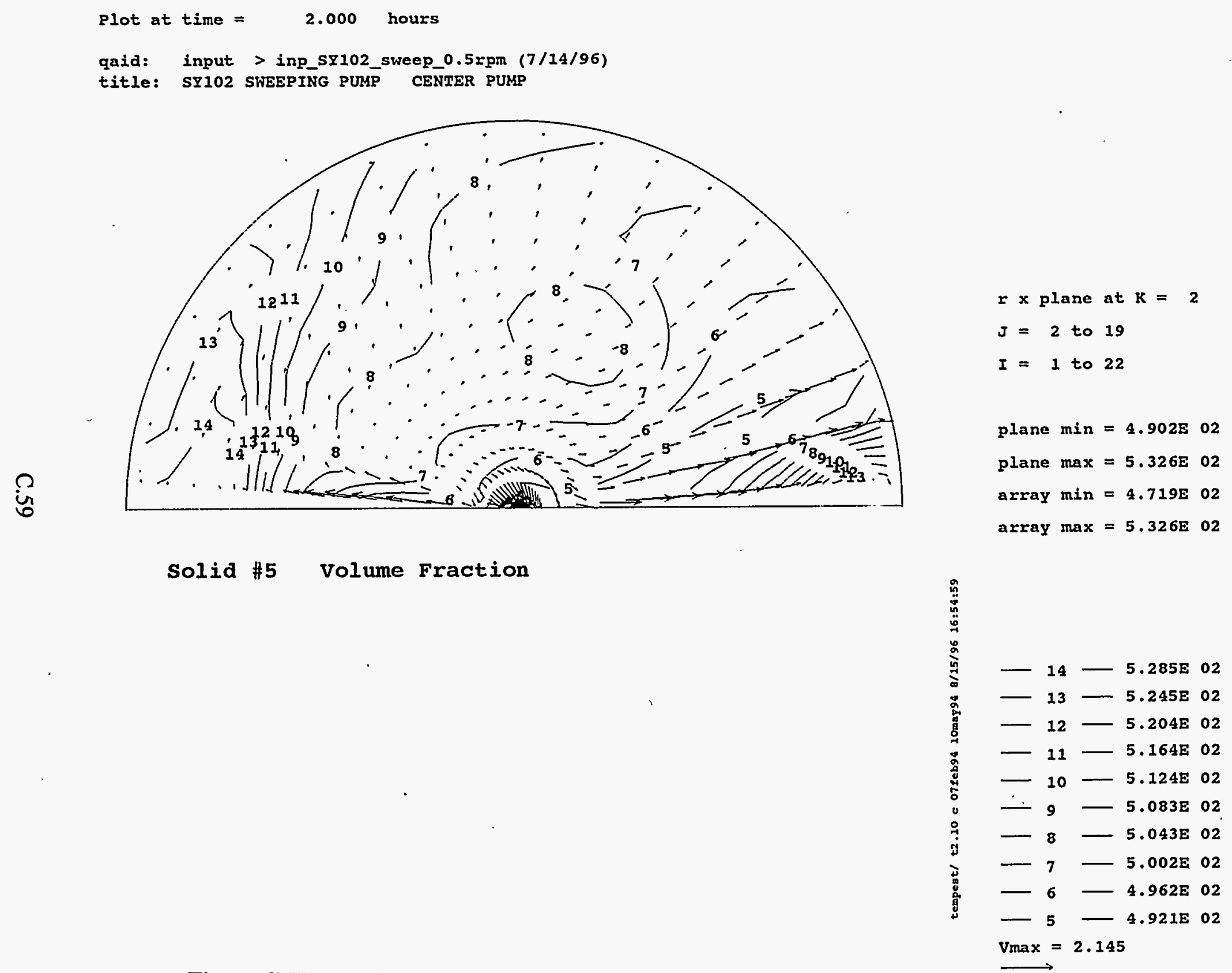

Figure C.59. Predicted Velocity $(\mathrm{m} / \mathrm{s})$ and Horizontal Distribution of Solid 5 Volume Fraction on the Tank Bottom at Two Simulation Hours for Case 5 


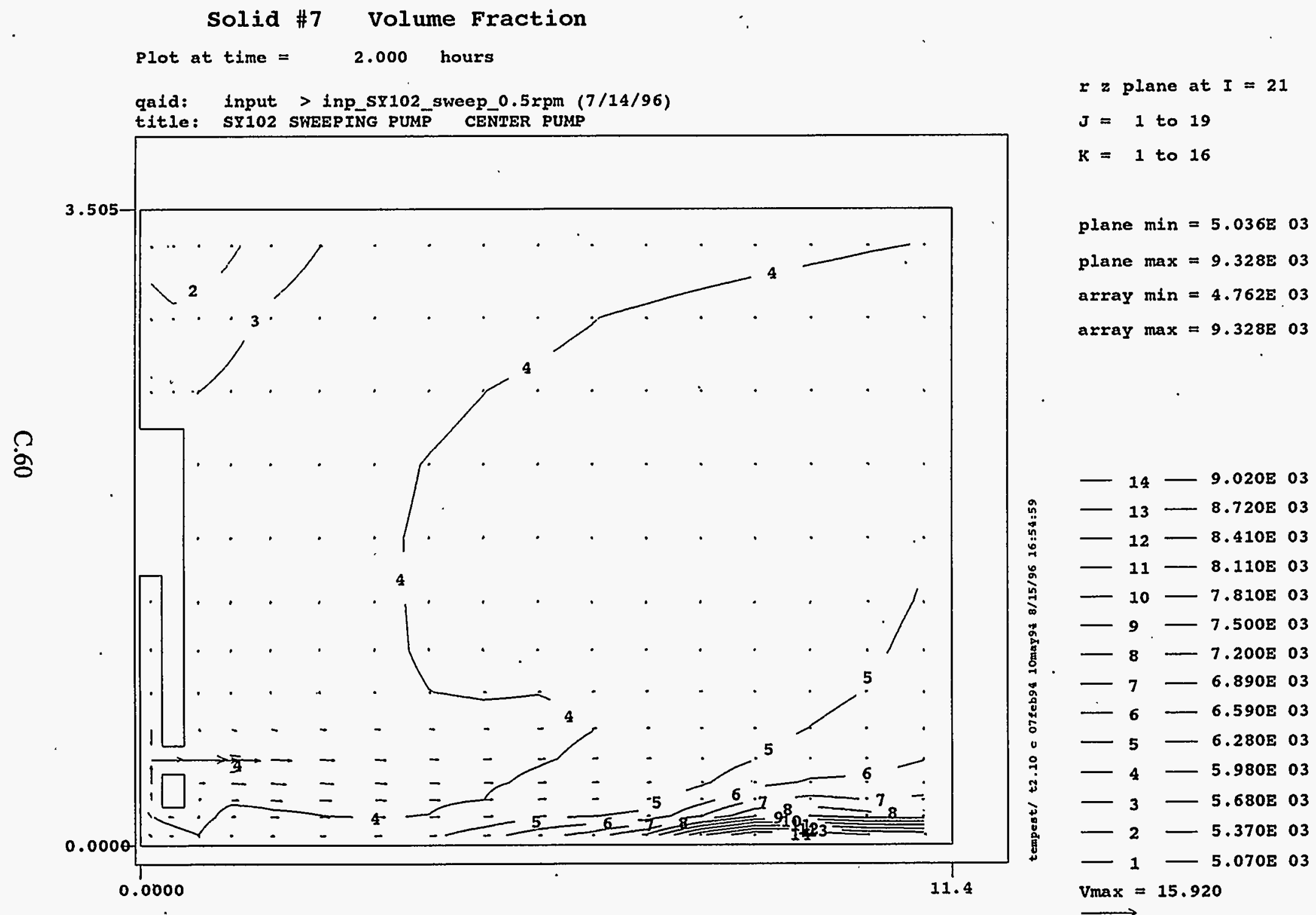

Figure C.60. Predicted Velocity $(\mathrm{m} / \mathrm{s})$ and Distribution of Solid 7 Volume Fraction on Vertical Plane 21 ( 9 o'clock position) at Two Simulation Hours for Case 5 


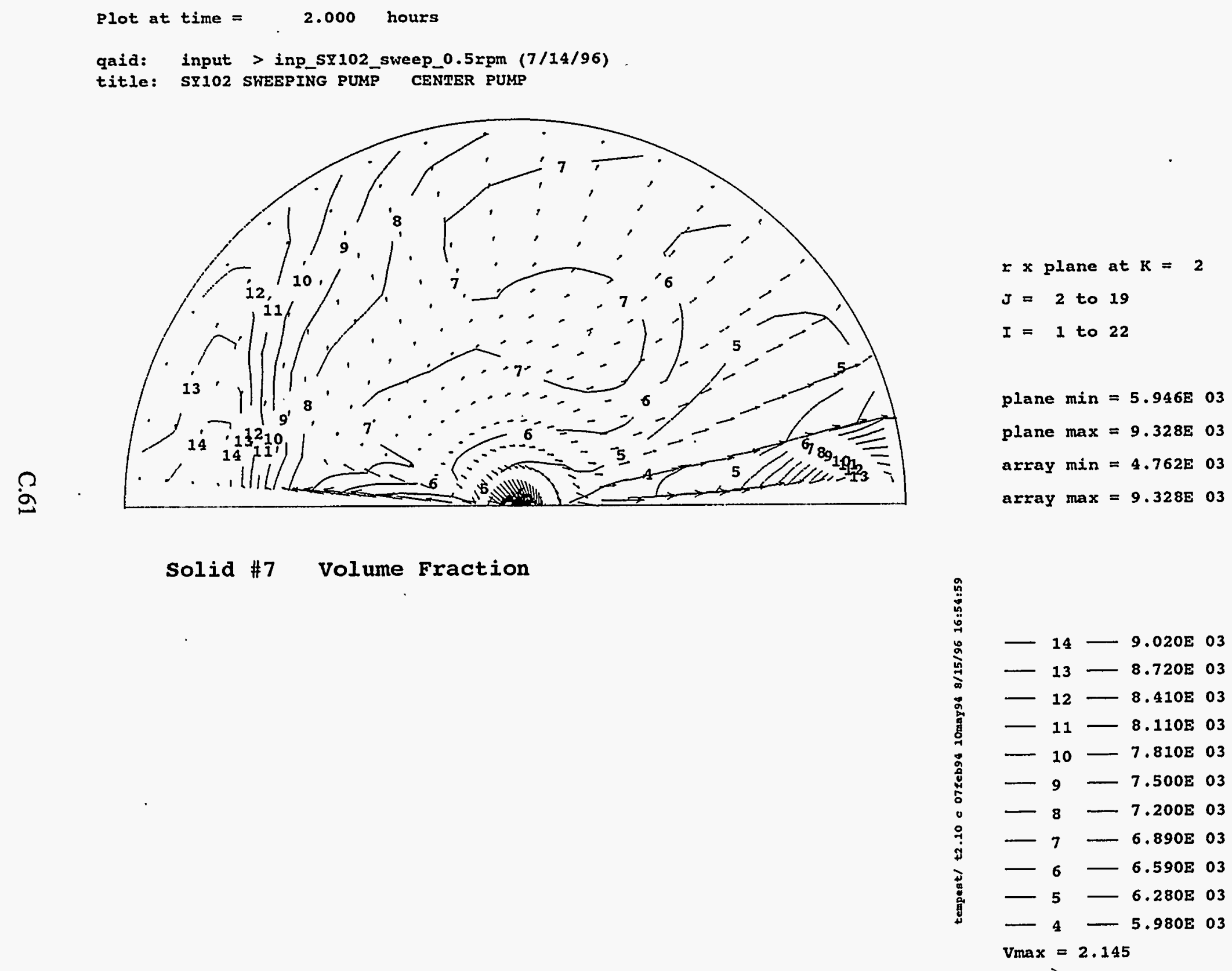

Figure C.61. Predicted Velocity $(\mathrm{m} / \mathrm{s})$ and Horizontal Distribution of Solid 7 Volume Fraction on the Tank Bottom at Two Simulation Hours for Case 5 


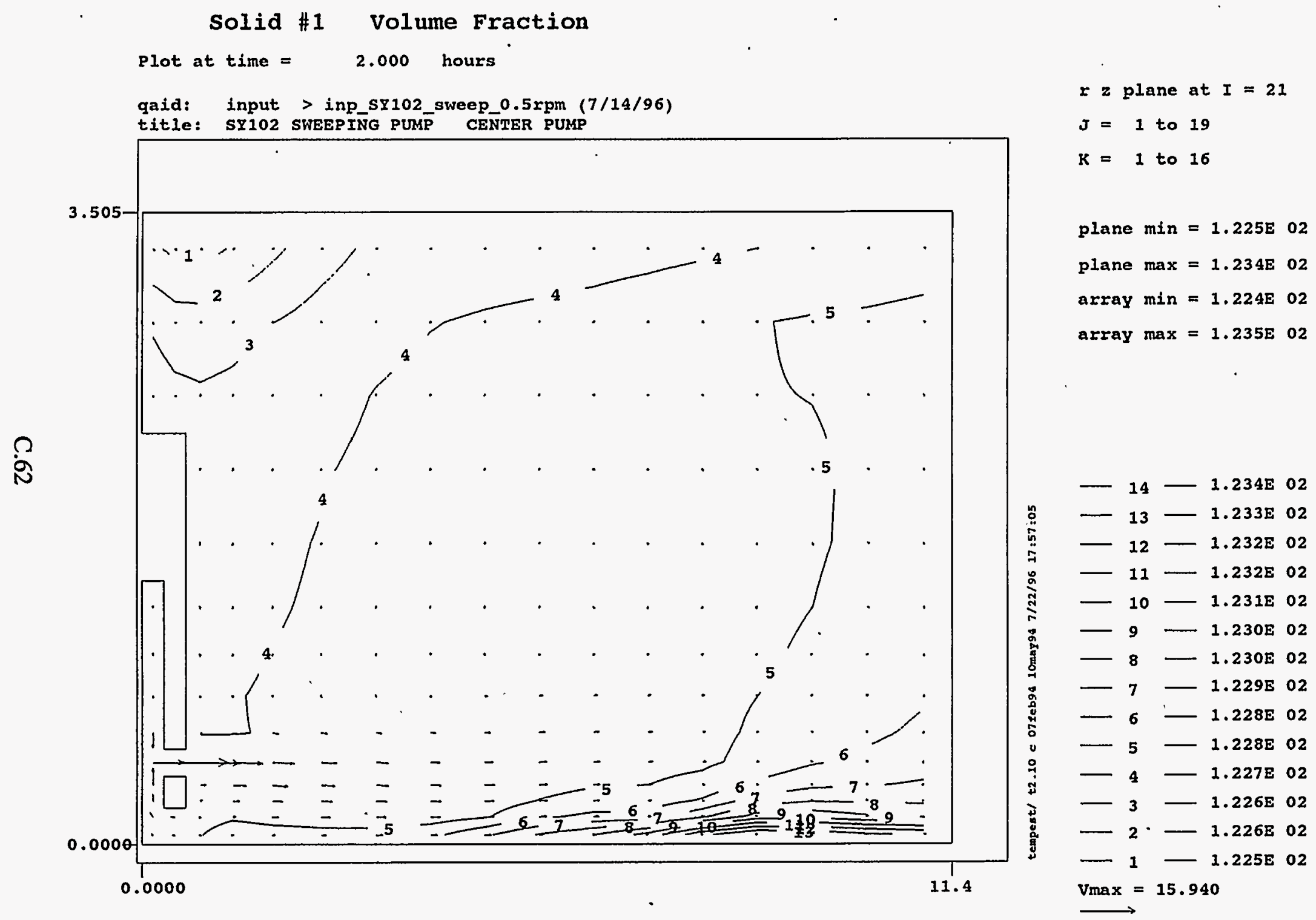

Figure C.62. Predicted Velocity $(\mathrm{m} / \mathrm{s})$ and Distribution of Solid 1 Volume Fraction on Vertical Plane 21 (9 o'clock position) at Two Simulation Hours for Case 6 


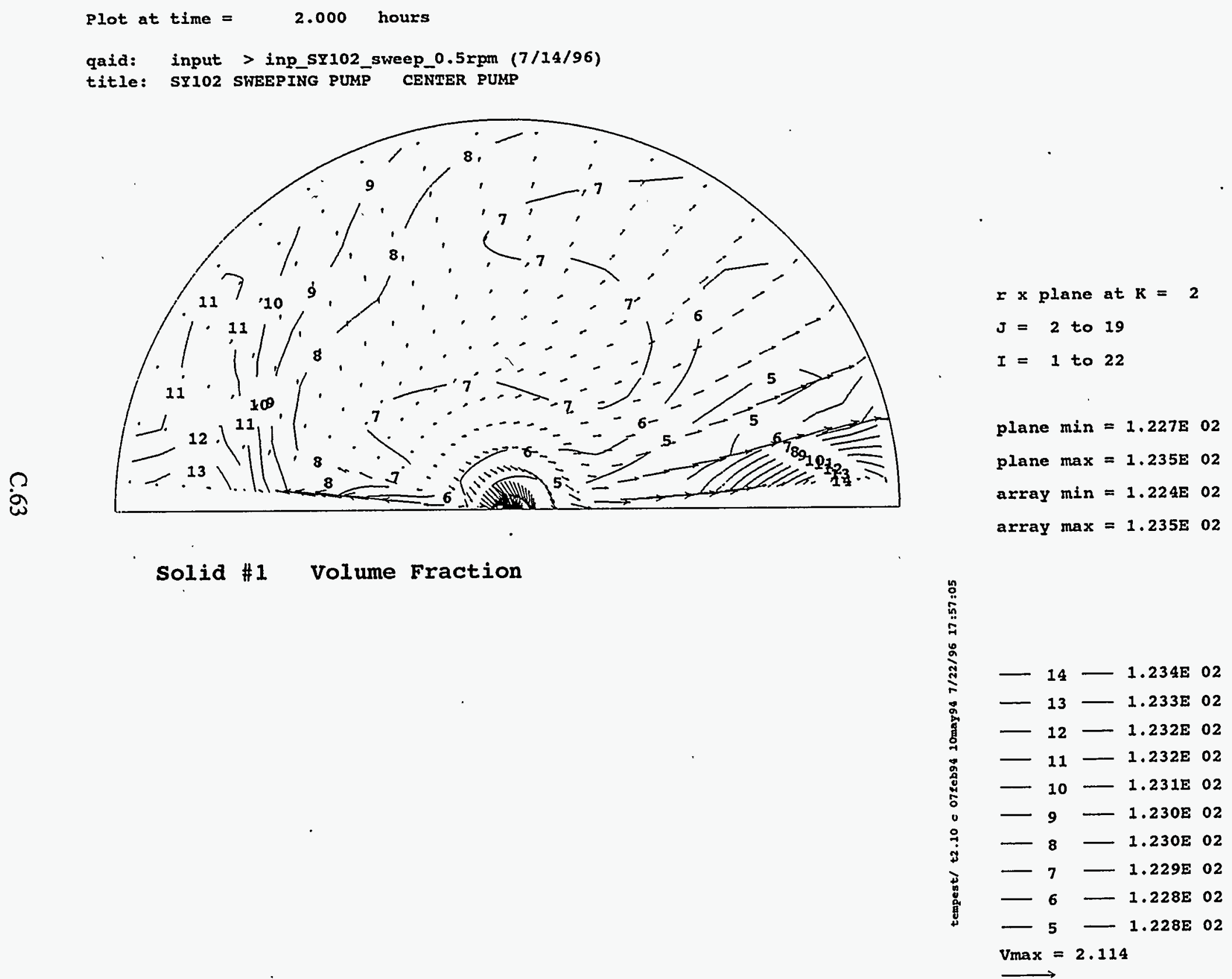

Figure C.63. Predicted Velocity $(\mathrm{m} / \mathrm{s})$ and Horizontal Distribution of Solid 1 Volume Fraction on the Tank Bottom at Two Simulation Hours for Case 6 


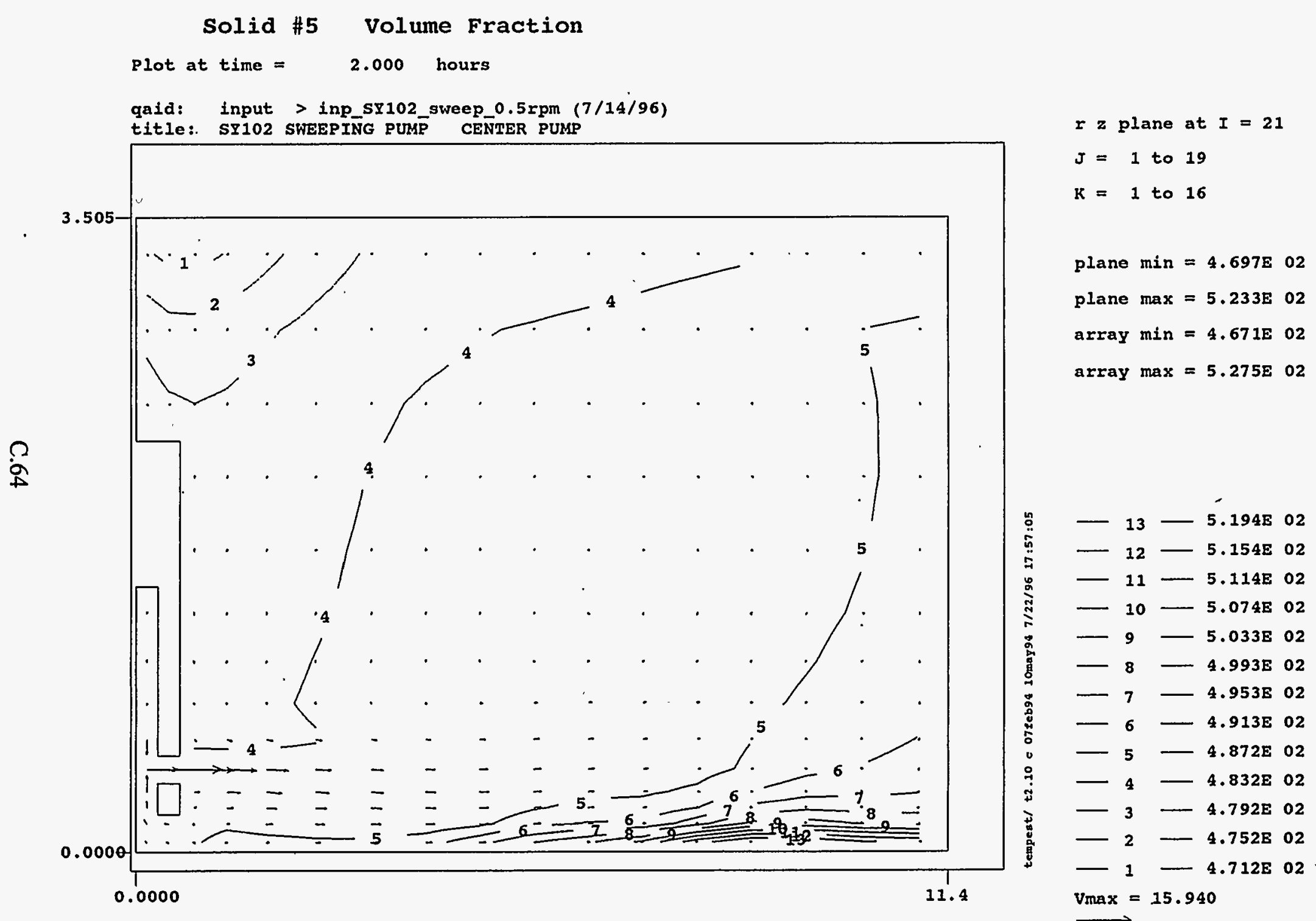

Figure C.64. Predicted Velocity $(\mathrm{m} / \mathrm{s})$ and Distribution of Solid 5 Volume Fraction on Vertical Plane 21 (9 o'clock position) at Two Simulation Hours for Case 6 
qaid: input > inp_sr102_sweep_0.5rpm $(7 / 14 / 96)$

title: SY102 SWEEPING PUMP CENTER PUMP

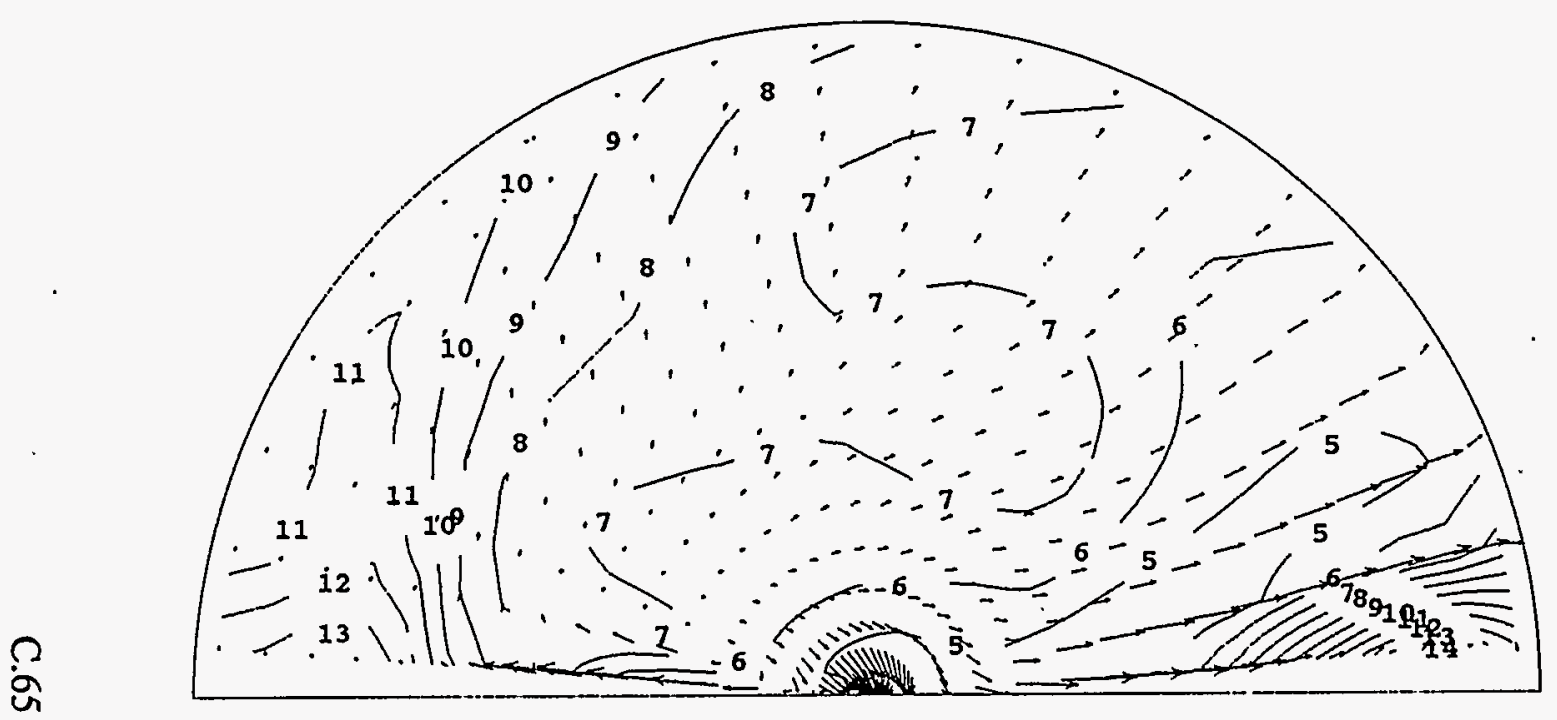

$x \times$ plane at $k=2$

$J=2$ to 19

$I=1$ to 22

plane $\min =4.844 \mathrm{E} 02$ plane max $=5.275 \mathrm{E} 02$ array $\mathrm{min}=4.671 \mathrm{E} 02$ áray $\max =5.275 \mathrm{E} 02$

\section{Solid \#5 Volume Fraction}

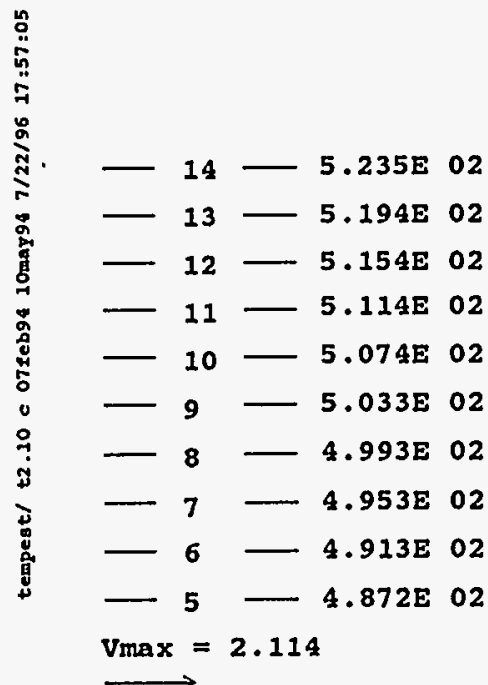

Figure C.65. Predicted Velocity $(\mathrm{m} / \mathrm{s})$ and Horizontal Distribution of Solid 5 Volume Fraction on the Tank Bottom at Two Simulation Hours for Case 6 


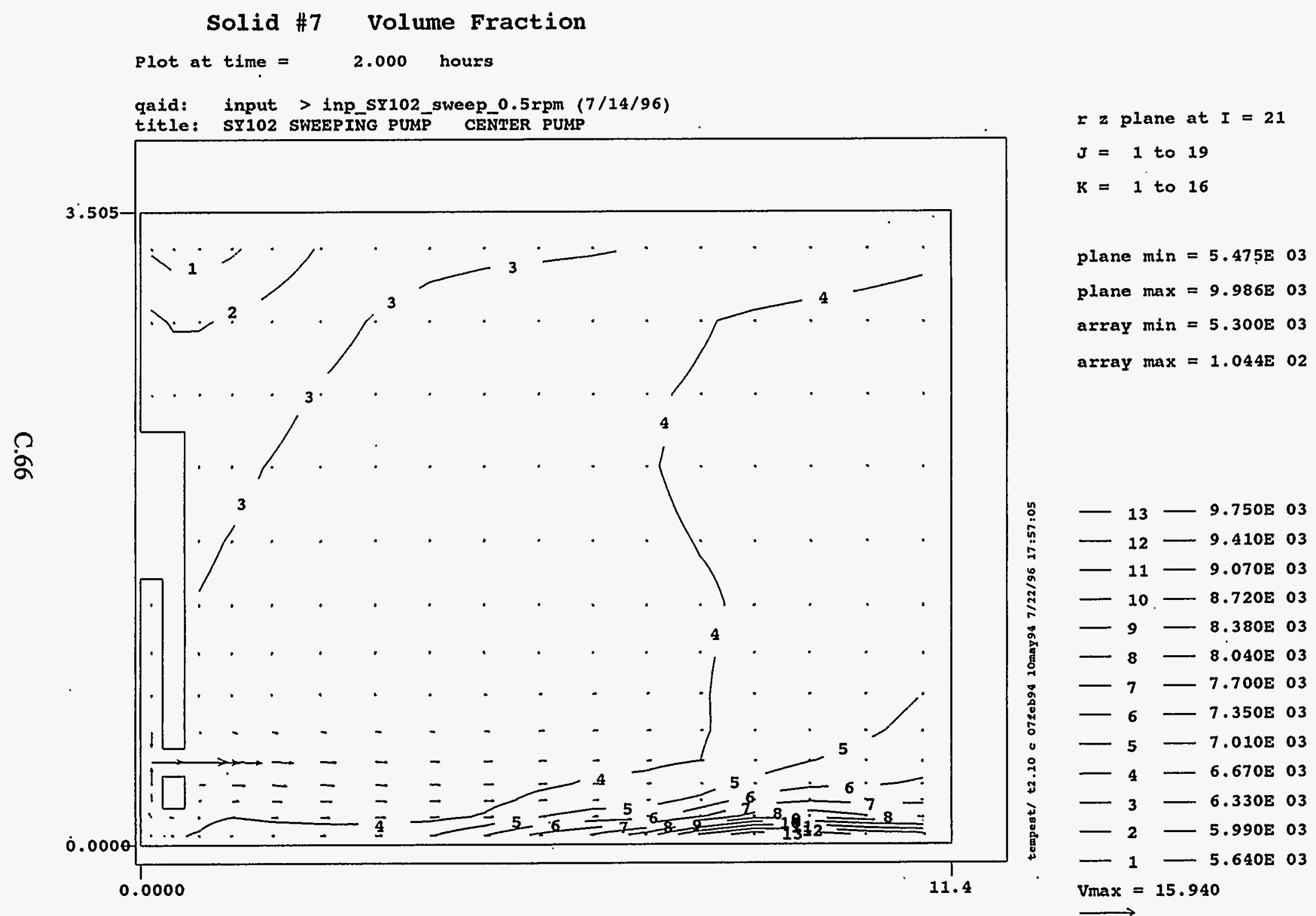

Figure C.66. Predicted Velocity $(\mathrm{m} / \mathrm{s})$ and Distribution of Solid 7 Volume Fraction on Vertical Plane 21 (9 o'clock position) at Two Simulation Hours for Case 6 
qaid: input > inp_sy102_sweep_0.5xpm $(7 / 14 / 96)$

title: SY102 SWEEPING PUMP CENTER PUMP

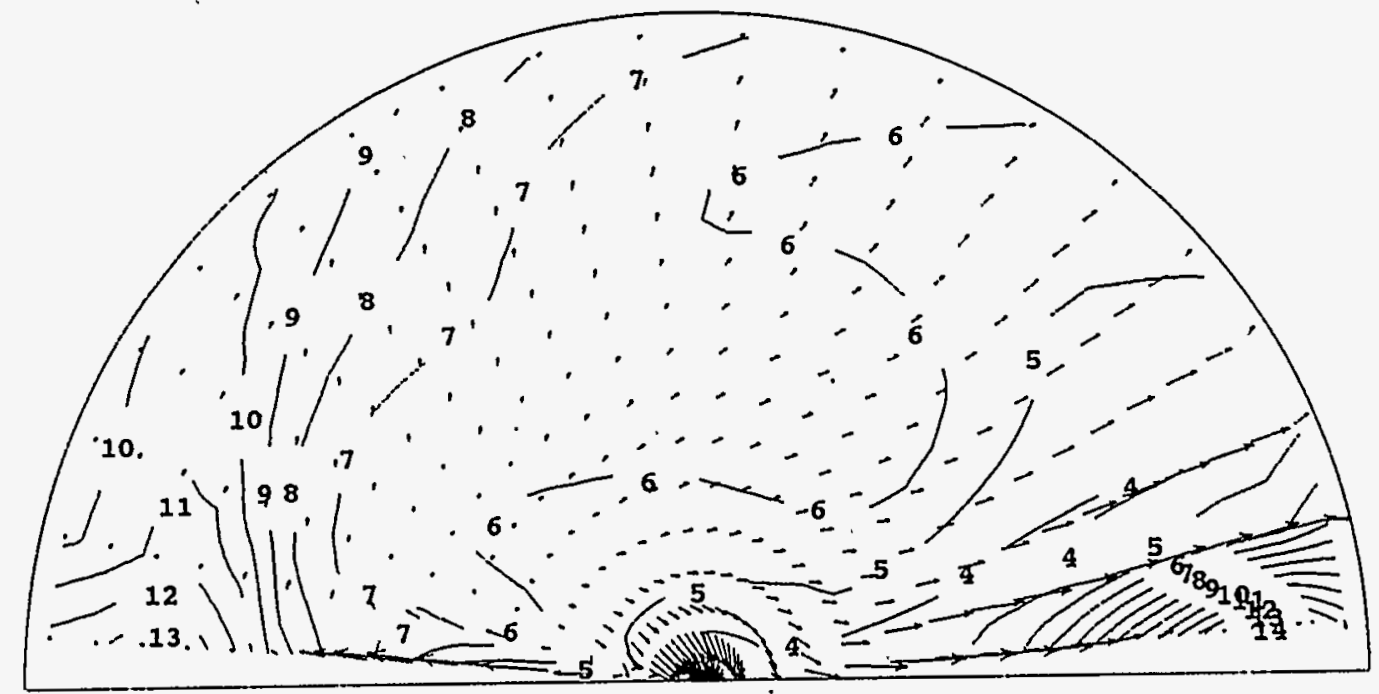

$r \times$ plane at $k=2$

$J=2$ to 19

$I=1$ to 22

\section{Solid \#7 Volume Fraction}

Figure C.67. Predicted Velocity $(\mathrm{m} / \mathrm{s})$ and Horizontal Distribution of Solid 7 Volume Fraction on the Tank Bottom at Two Simulation Hours for Case 6 


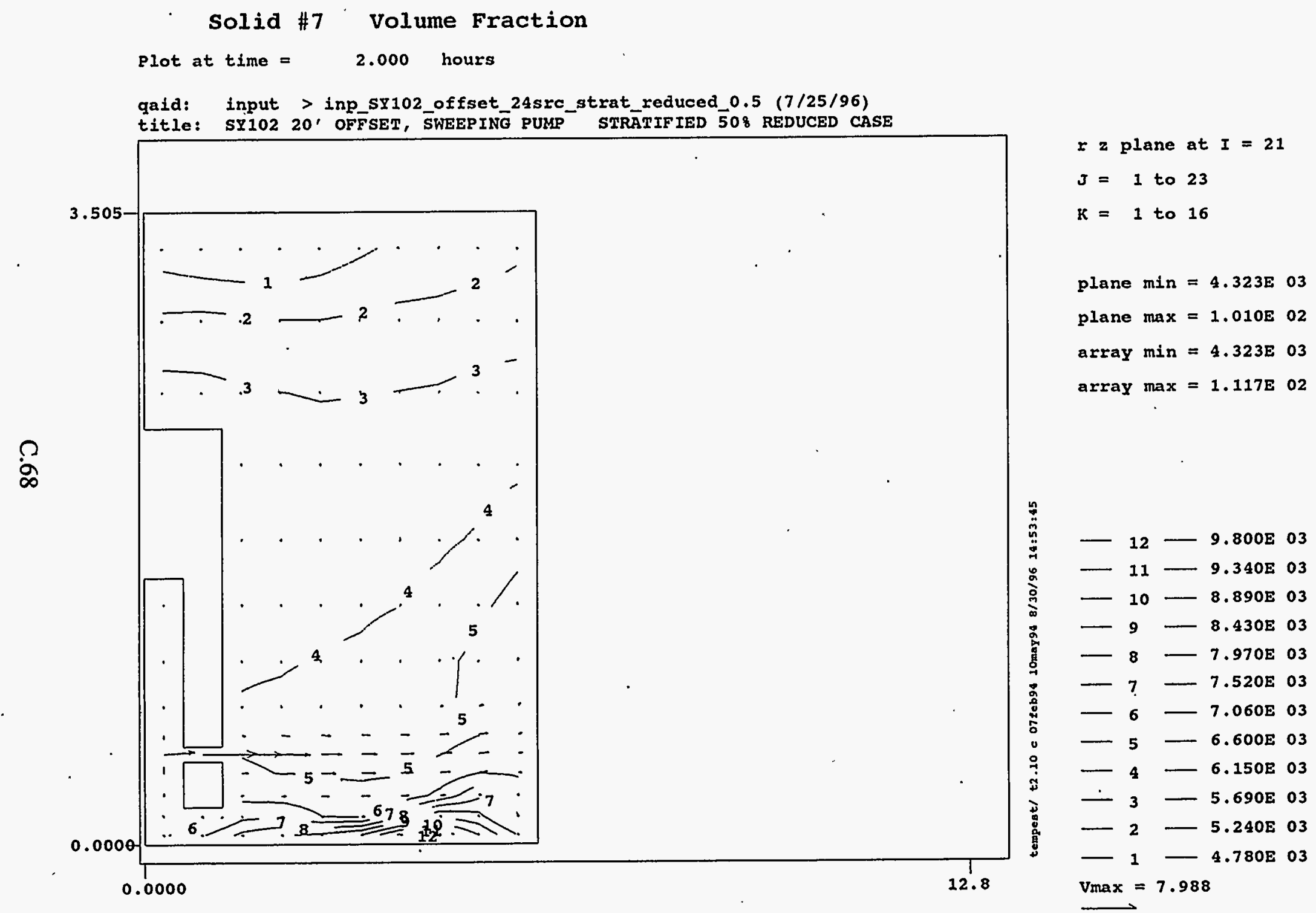

Figure C.68. Predicted Velocity $(\mathrm{m} / \mathrm{s})$ and Distribution of Solid 7 Volume Fraction on Vertical Plane 21 ( 9 o'clock position) at Two Simulation Hours for Case 8 
qaid: input $>$ inp_sY102_offset_24src_strat_reduced_0.5 (7/25/96)

title: sY102 20' OFFSET, SWEEPING PUMP STRATIFIED 508 REDUCED CASE

\section{Solid \#7 Volume Fraction}

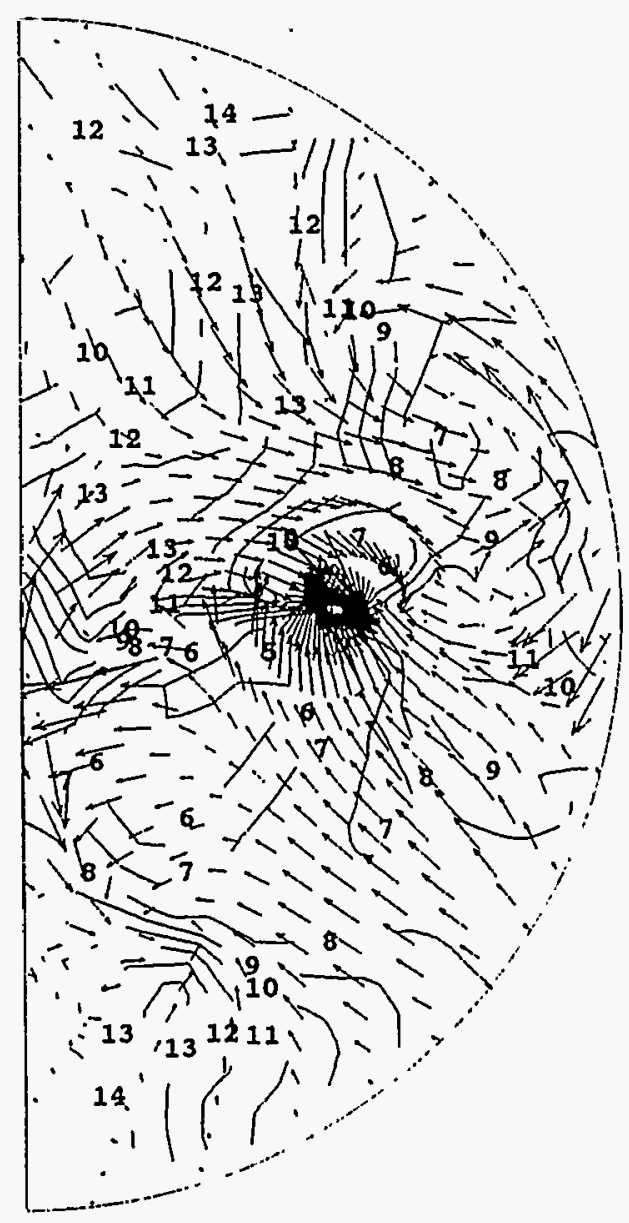

I $x$ plane at $K=2$

$J=2$ to 23

$I=1$ to 42

plane min $=6.369 \mathrm{E} 03$

plane $\max =1.117 \mathrm{E} 02$

array min $=4.323 \mathrm{E} 03$

array $\max =1.117 \mathrm{E} 02$

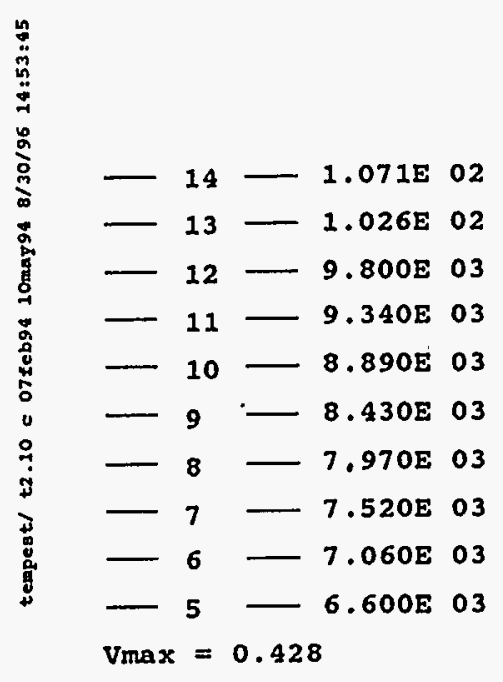

Figure C.69. Predicted Velocity $(\mathrm{m} / \mathrm{s})$ and Horizontal Distribution of Solid 7 Volume Fraction on the Tank Bottom at Two Simulation Hours for Case 8 


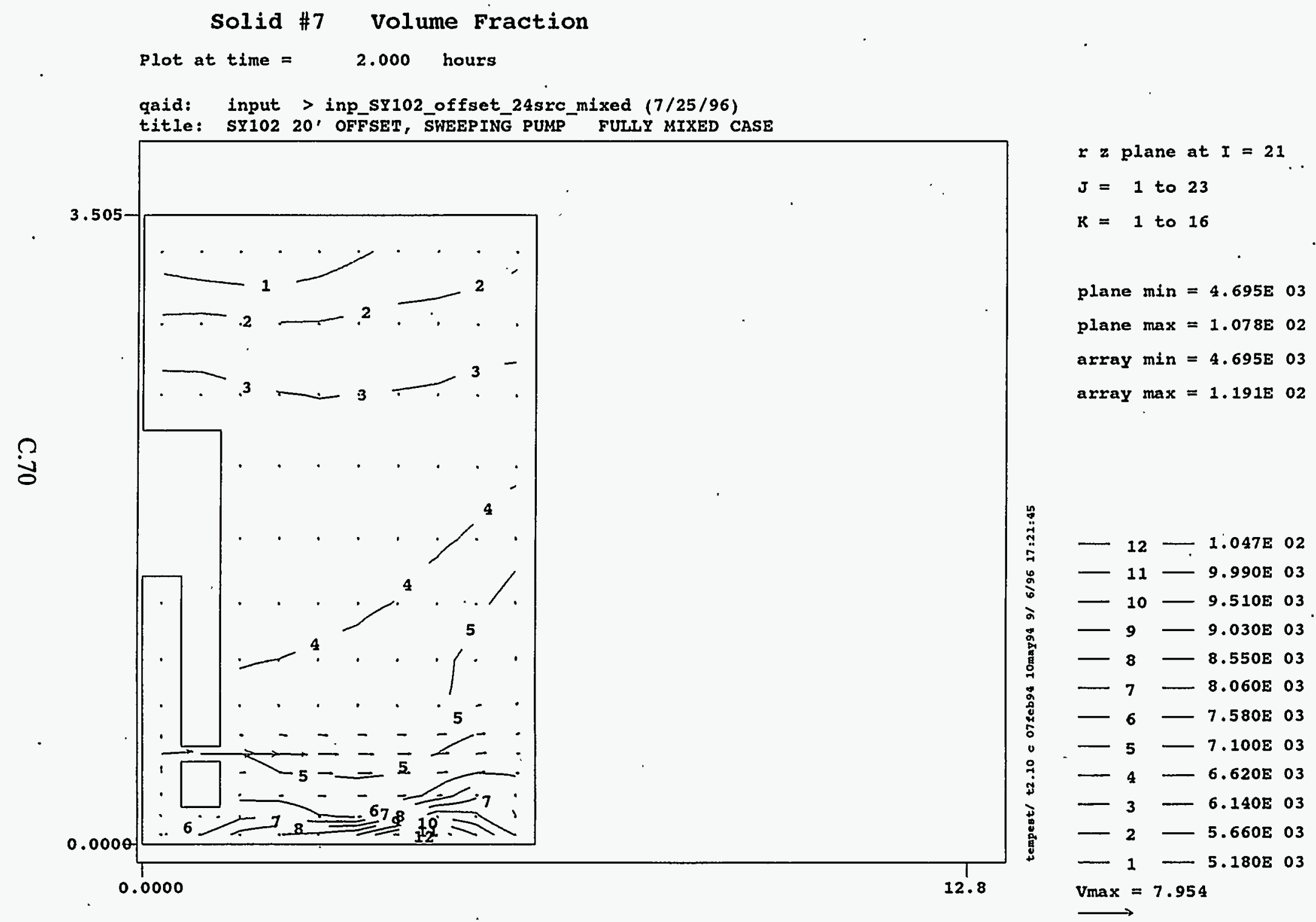

Figure C.70. Predicted Velocity $(\mathrm{m} / \mathrm{s})$ and Distribution of Solid 7 Volume Fraction on Vertical Plane 21 (9 o'clock position) at Two Simulation Hours for Case 9 
qaid: input $>$ inp SY102 offset_24sIc_mixed (7/25/96)

title: SY102 20' OFFSET, SWEEPING PUMP FULLY MIXED CASE

\section{Solid \#7 Volume Fraction}

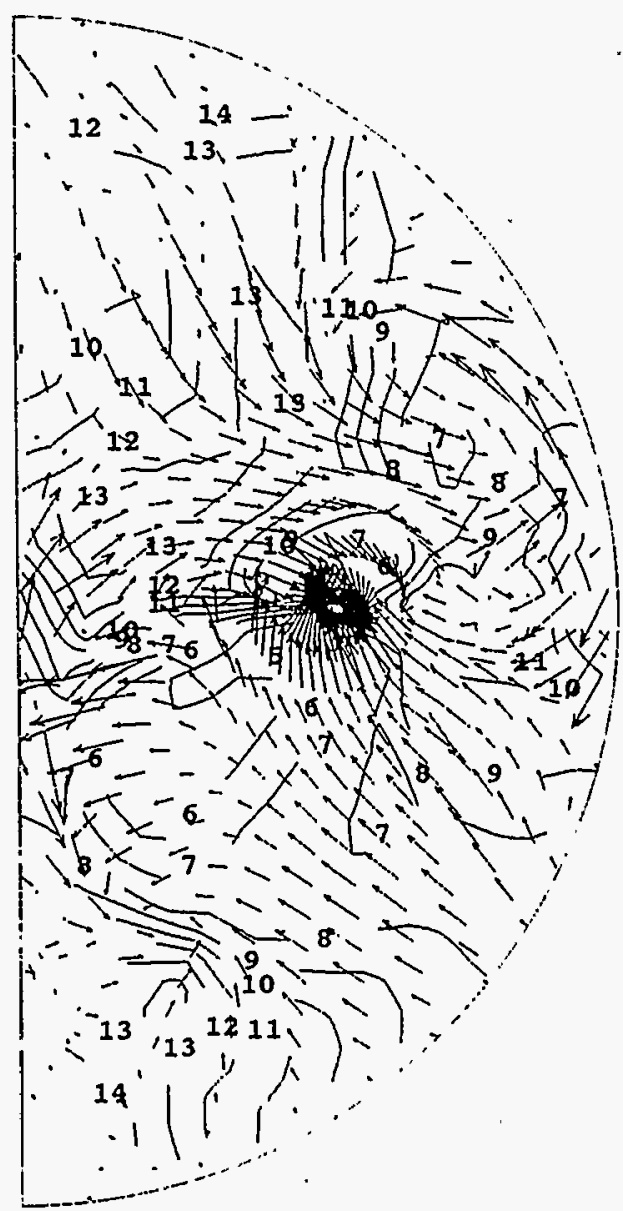

I $x$ plane at $k=2$

$J=2$ to 23

$I=I$ to 42

plane $\min =6.861 \mathrm{E} 03$

plane max $=1.191 \mathrm{E} 02$

array $\min =4.695 \mathrm{E} 03$

array $\max =1.191 \mathrm{E} 02$

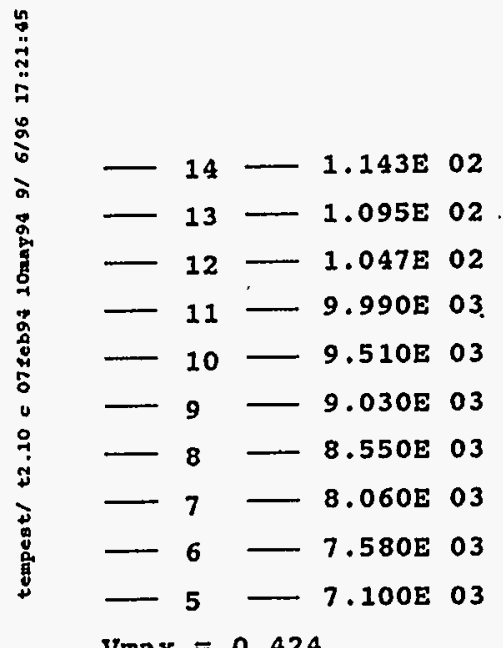

$V \max =0.424$

Figure C.71. Predicted Velocity $(\mathrm{m} / \mathrm{s})$ and Horizontal Distribution of Solid 7 Volume Fraction on the Tank Bottom at Two Simulation Hours for Case 9 


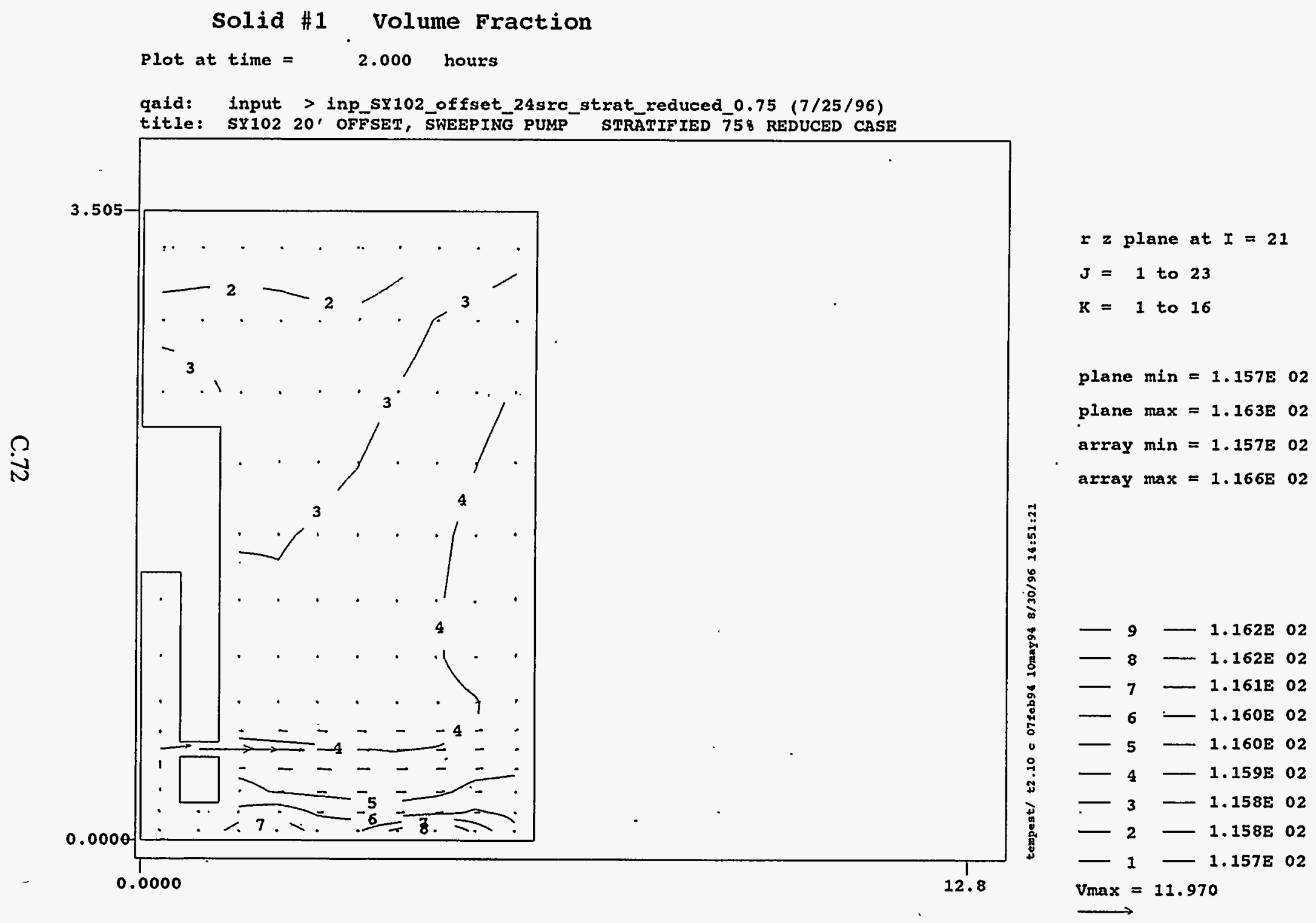

Figure C.72. Predicted Velocity $(\mathrm{m} / \mathrm{s})$ and Distribution of Solid 1 Volume Fraction on Vertical Plane 21 (9 o'clock position) at Two Simulation Hours for Case 7 


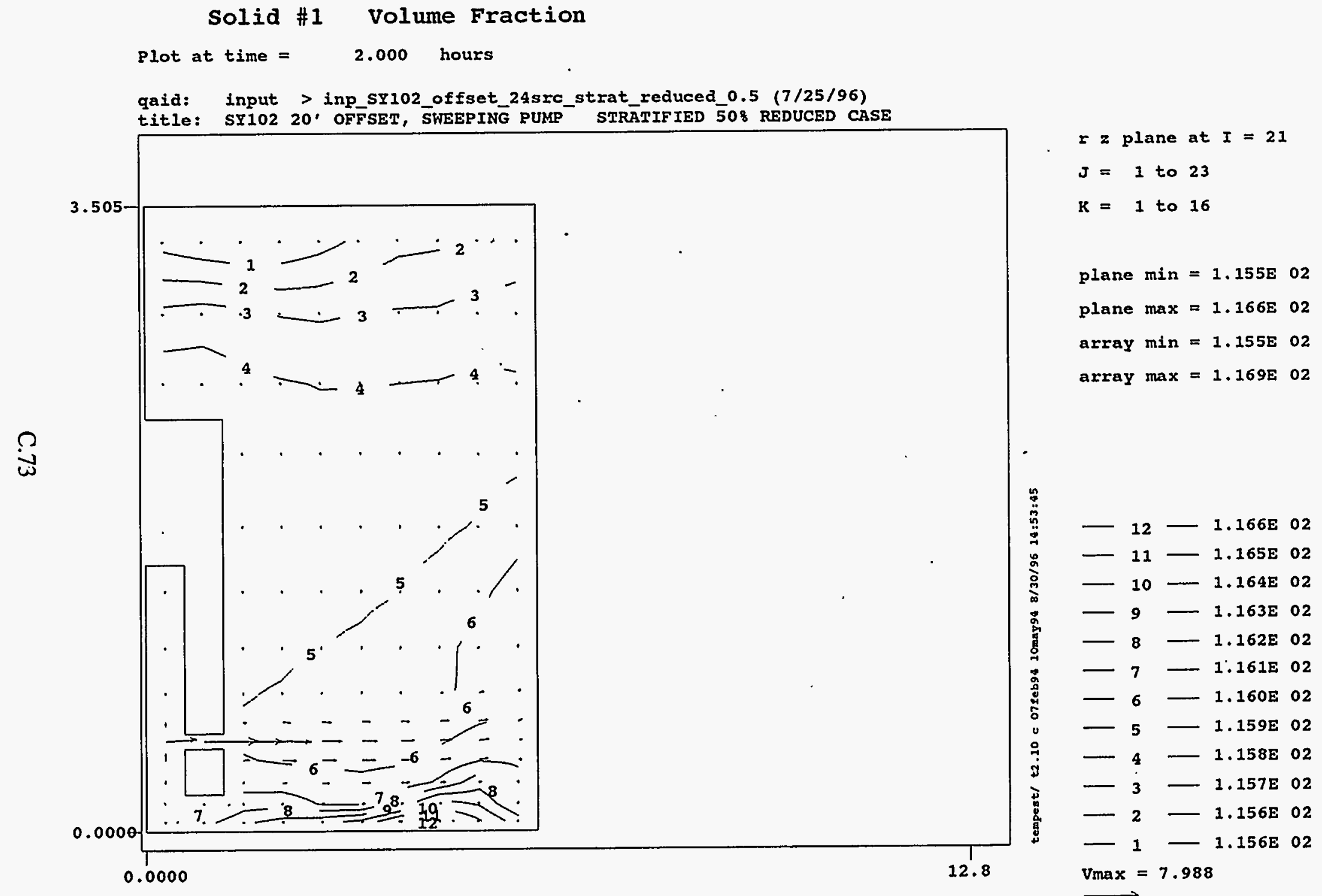

Figure C.73. Predicted Velocity $(\mathrm{m} / \mathrm{s})$ and Distribution of Solid 1 Volume Fraction on Vertical Plane 21 (9 o'clock position) at 'Two Simulation Hours for Case 8 


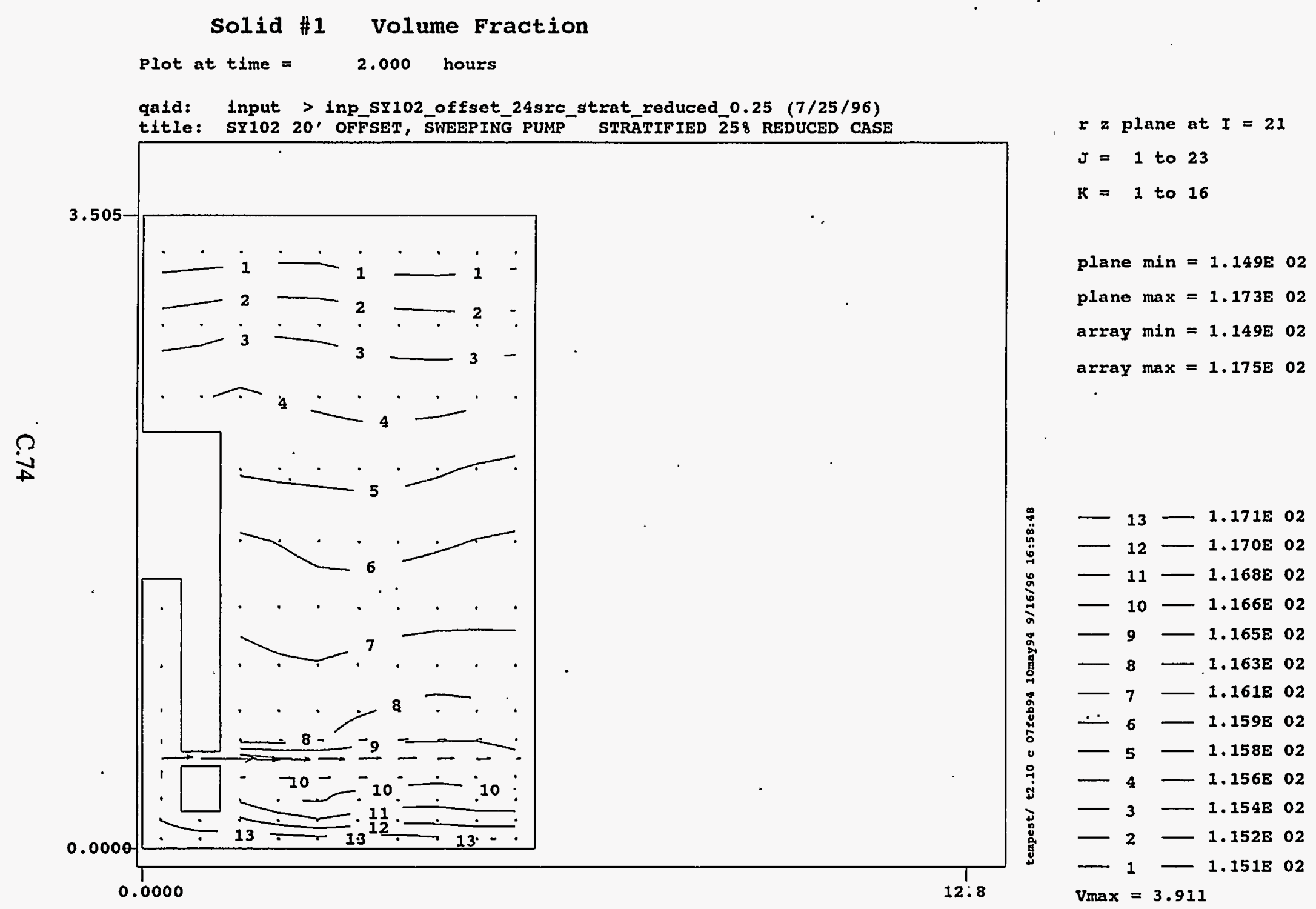

Figure C.74. Predicted Velocity (m/s) and Distribution of Solid 1 Volume Fraction on Vertical Plane 21 (9 o'clock position) at Two Simulation Hours for Case 10 


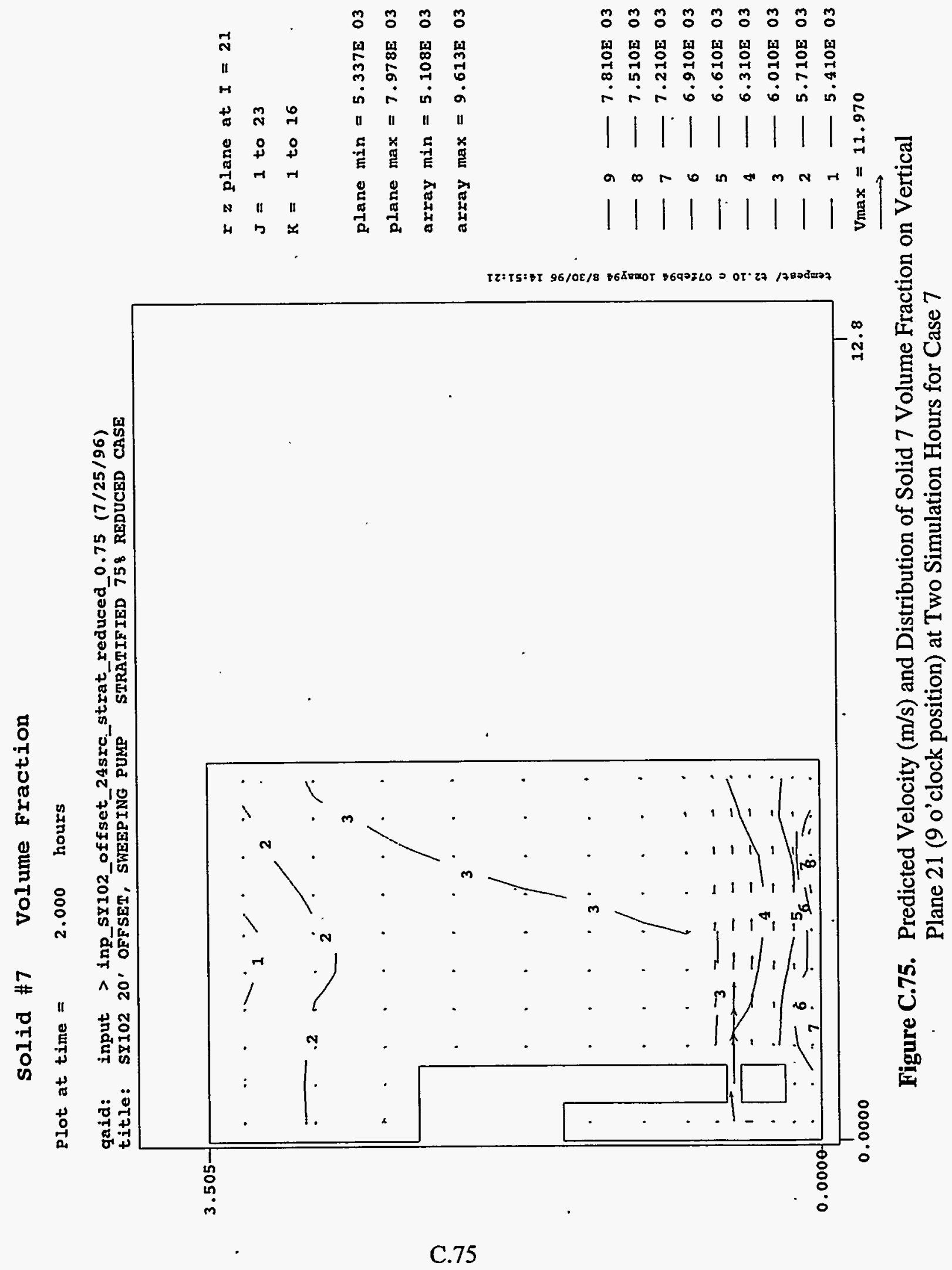


qaid: input > inp_SY102_offset_24sre_strat_reduced_0.75 (7/25/96)

title: SY102 20' OFFSET, SWEEPING PUMP STRATIFIED $75 \%$ REDUCED CASE

\section{Solid \#7 Volume Fraction}

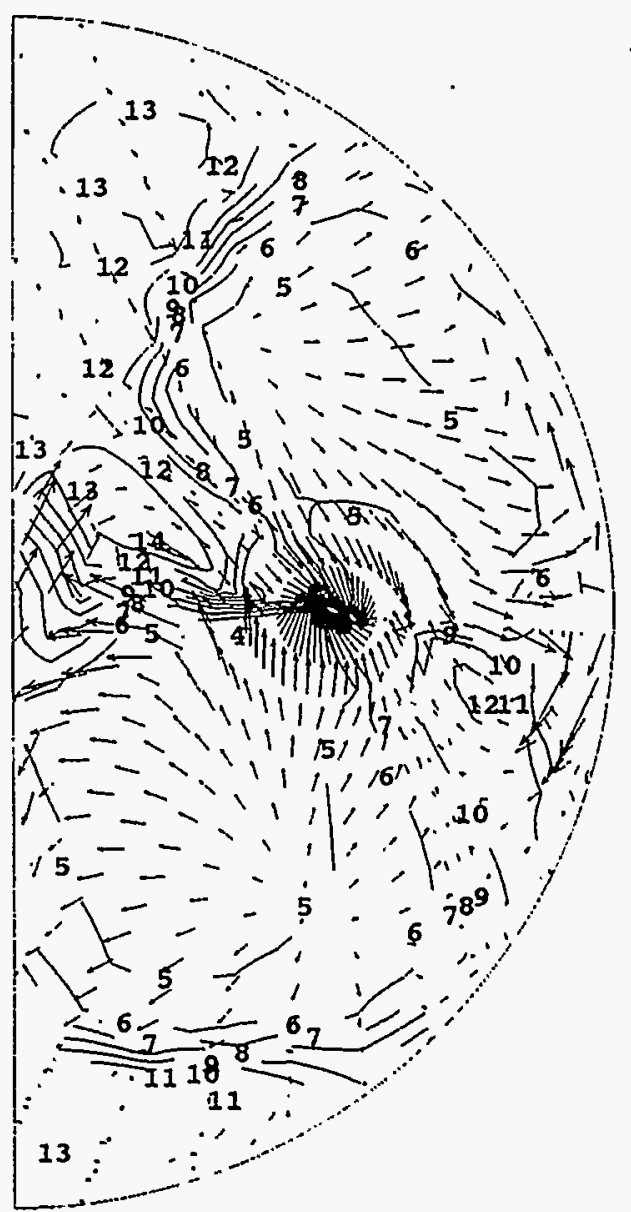

$x$ plane at $k=2$

$J=2$ to 23

$I=1$ to 42

plane $\min =6.271 \mathrm{E} 03$ plane $\max =9.613 \mathrm{E} 03$ array $\min =5.108 \mathrm{E} 03$ array $\max =9.613 \mathrm{E} 03$

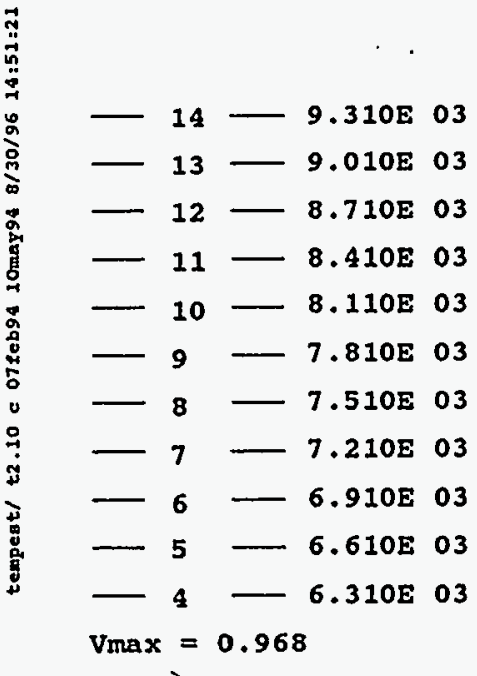

Figure C.76. Predicted Velocity $(\mathrm{m} / \mathrm{s})$ and Horizontal Distribution of Solid 7 Volume Fraction on the Tank Rnttrm at Tixn Simulatinn Hours for Cace 7 


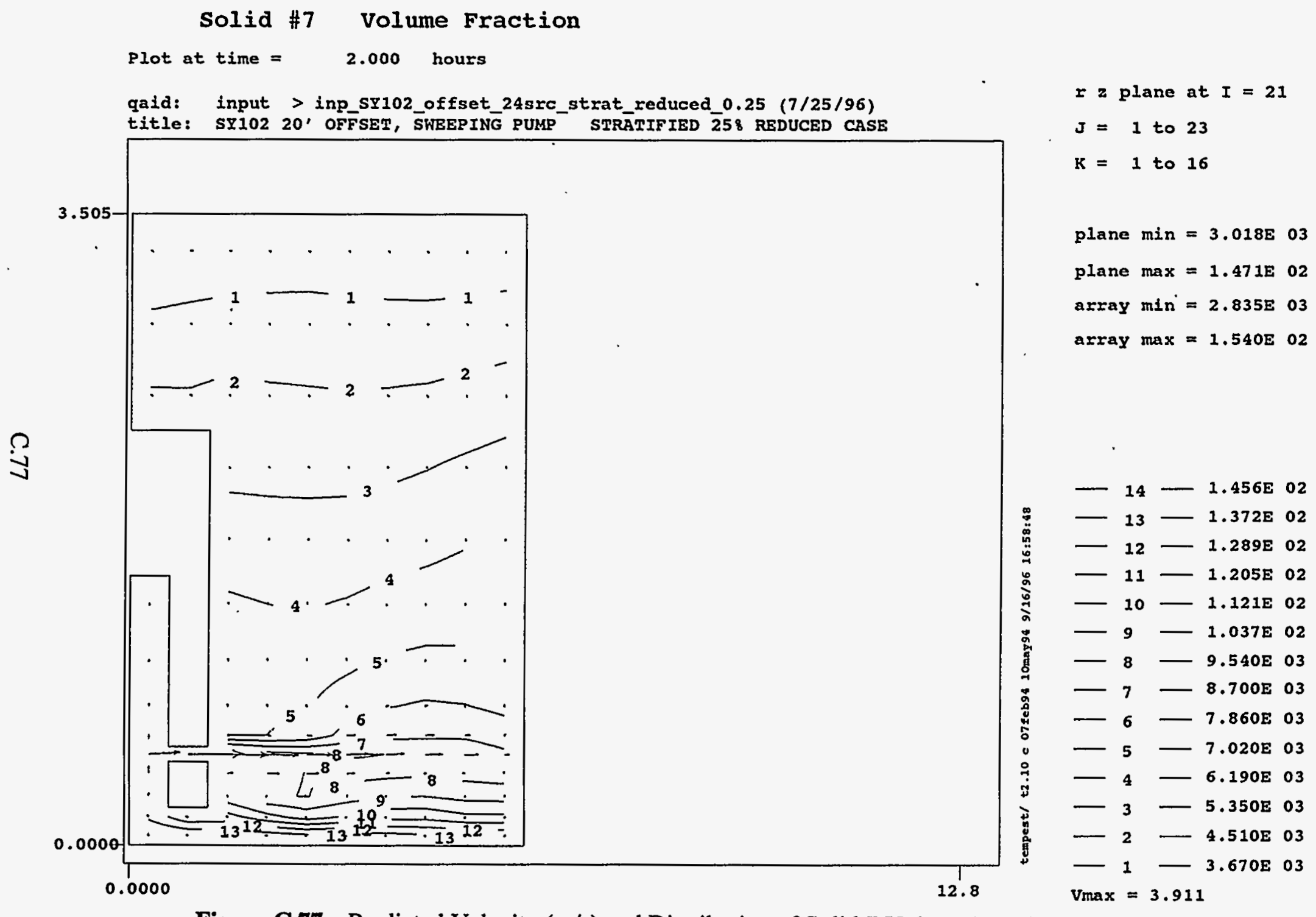

Figure C.77. Predicted Velocity $(\mathrm{m} / \mathrm{s})$ and Distribution of Solid 7 Volume Fraction on Vertical Plane 21 ( 9 o'clock position) at Two Simulation Hours for Case 10 
qaid: input > inp_sy102_offset_24sre_strat_reduced_0.25(7/25/96)

title: SY102 20, OFFSET, SWEEPING PUMP ${ }^{\circ}$ STRATIFIED 258 REDUCED CASE

Solid \#7 . Volume Fraction

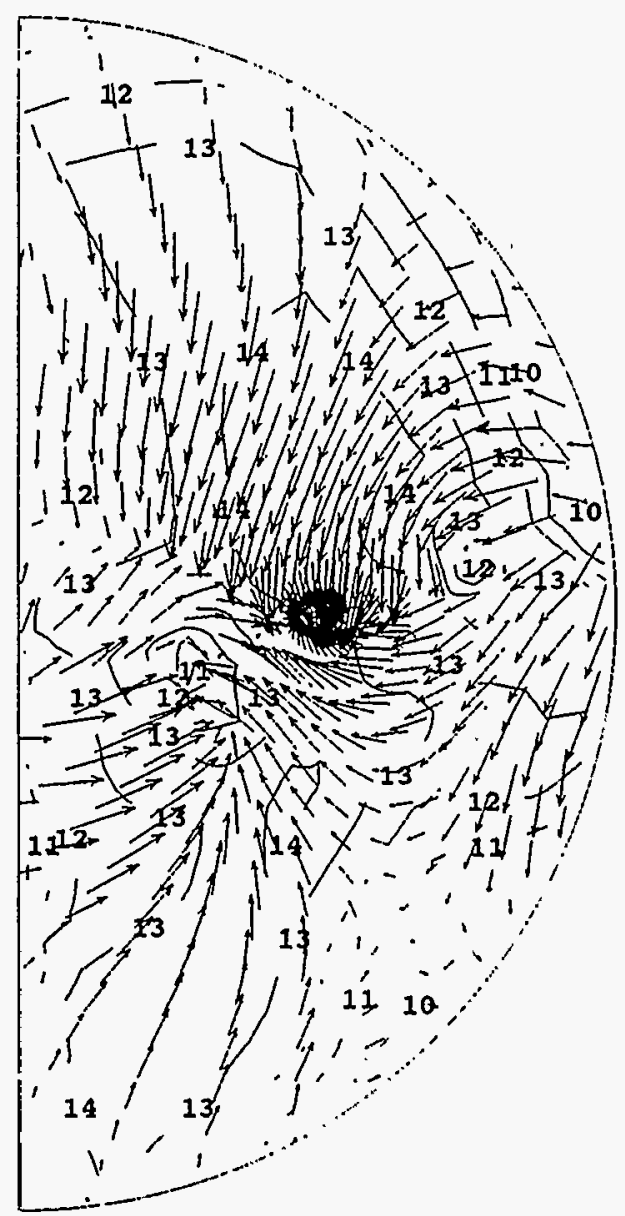

$r \mathrm{x}$ plane at $K=2$

$J=2$ to 23

$I=1$ to 42

plane $\min =1.033 \mathrm{E} 02$

plane $\max =1.540 \mathrm{E} 02$

array $\min =2.835 \mathrm{E} 03$

array $\max =1.540 \mathrm{E} 02$

$-14-1.456 \mathrm{E} 02$

$-13-1.372 \mathrm{E} 02$

$-12-1.289 \mathrm{E} 02$

$-11-1.205 \mathrm{E} 02$

$-10-1.121 E 02$

$-9-1.037 \mathrm{E} 02$

$V \max =0.221$

Figure C.78. Predicted Velocity $(\mathrm{m} / \mathrm{s})$ and Horizontal Distribution of Solid 7 Volume Fraction on the Tank Bottom at Two Simulation Hours for Case 10 


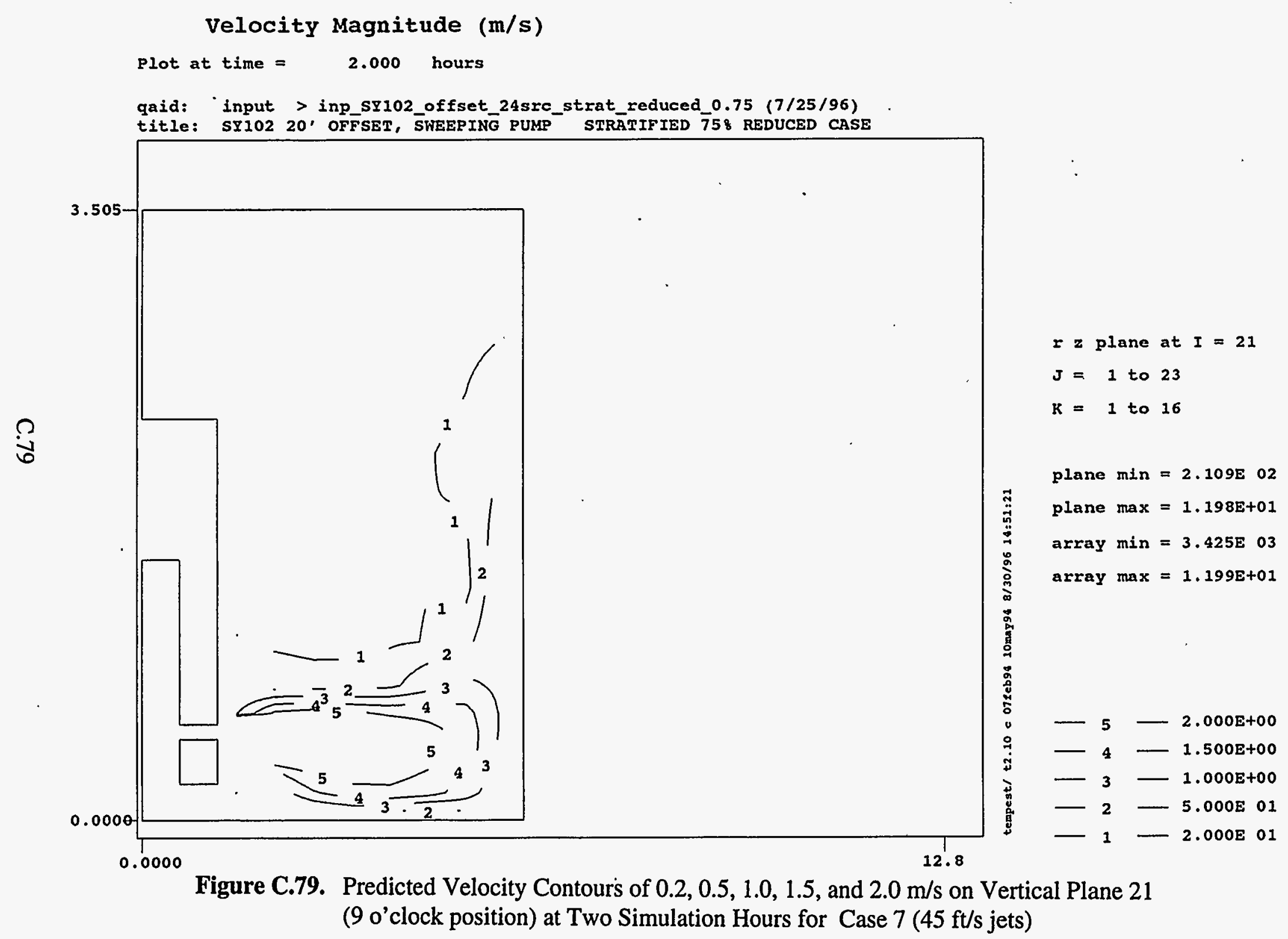




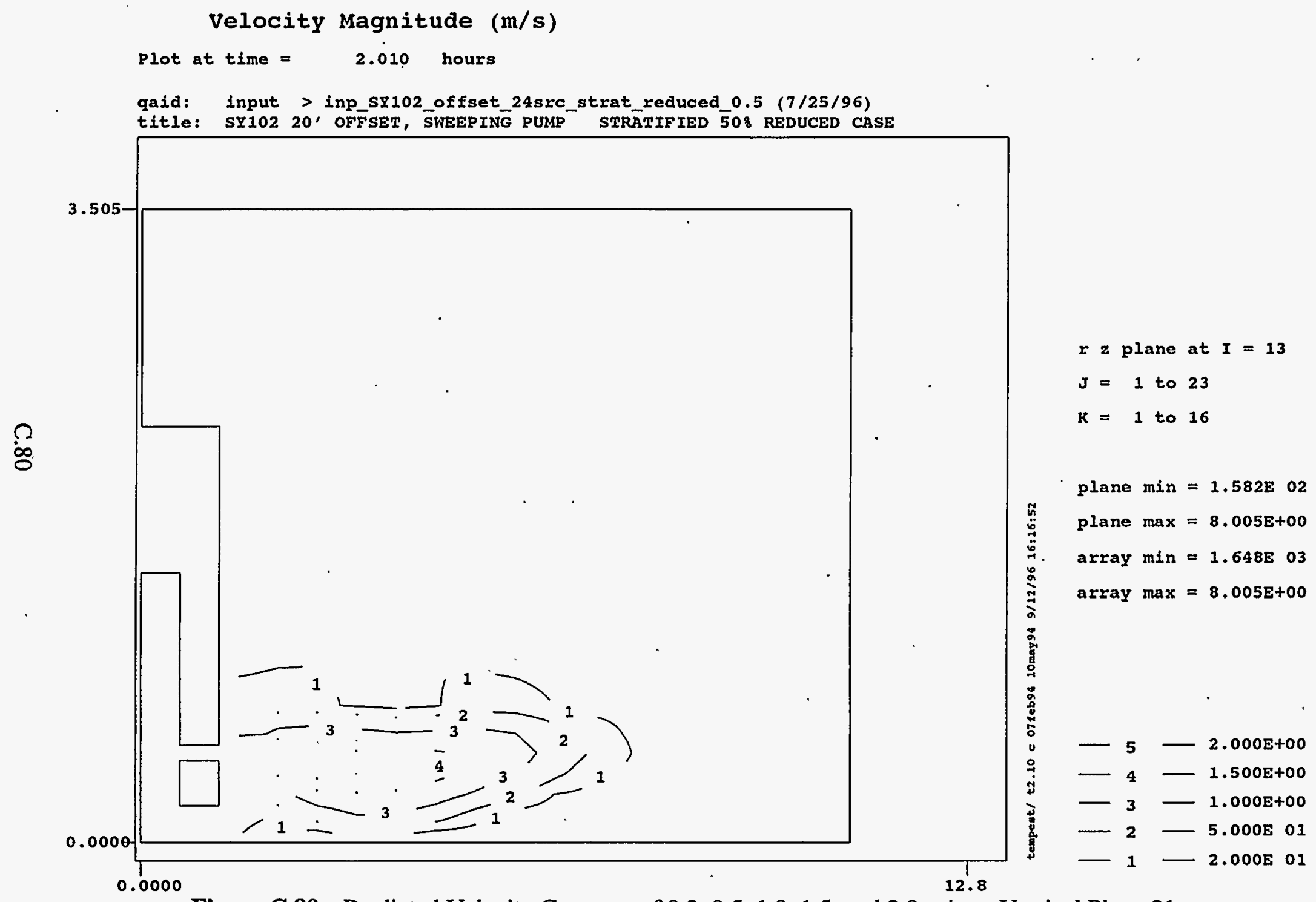

Figure C.80. Predicted Velocity Contours of $0.2,0.5,1.0,1.5$, and $2.0 \mathrm{~m} / \mathrm{s}$ on Vertical Plane 21 ( 9 o'clock position) at Two Simulation Hours for Case 8 ( $30 \mathrm{ft} / \mathrm{s}$ jets) 


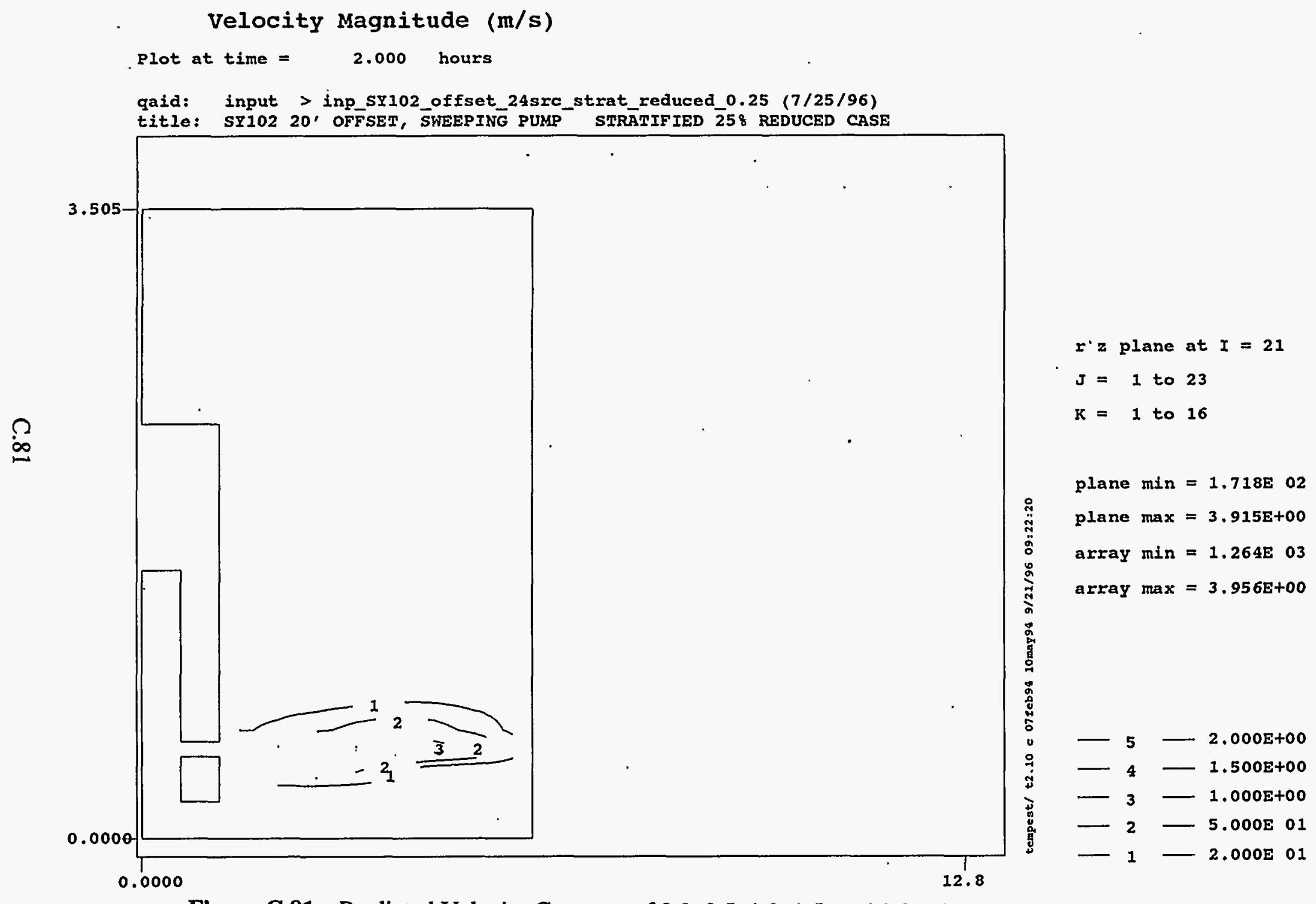

Figure C.81. Predicted Velocity Contours of $0.2,0.5,1.0,1.5$, and $2.0 \mathrm{~m} / \mathrm{s}$ on Vertical Plane 21

( 9 o'clock position) at Two Simulation Hours for Case 10 (15 ft/s jets) 
$0.2 \mathrm{~m} / \mathrm{s}, 26 \mathrm{~Pa}$

$\mathrm{r}=8 \mathrm{~m}(25 \mathrm{ft})$

$0.5 \mathrm{~m} / \mathrm{s}, 160 \mathrm{~Pa}$

$\mathrm{r}=7 \mathrm{~m}(22 \mathrm{ft})$

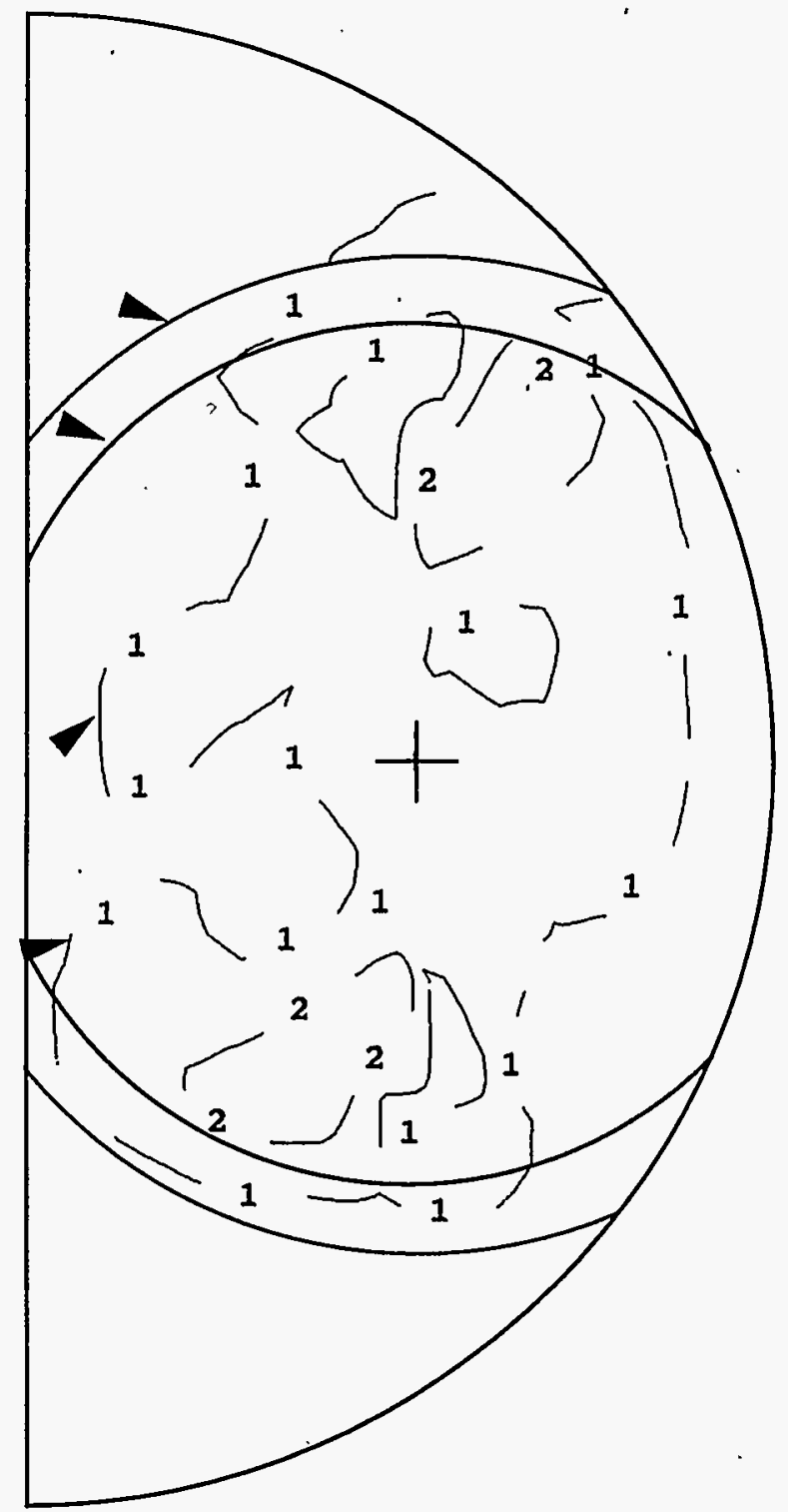

Figure C.82. Estimated Tank Bottom Areas for Possible Sludge Mobilization at Different Velocity and Yield Stress Values for Case 7 (45 ft/s jets) 
$0.2 \mathrm{~m} / \mathrm{s}, 26 \mathrm{~Pa}$

$\mathrm{r}=7 \mathrm{~m}(23 \mathrm{ft})$

$0.5 \mathrm{~m} / \mathrm{s}, 160 \mathrm{~Pa}$

$\mathrm{r}=6 \mathrm{~m}(18 \mathrm{ft})$

م

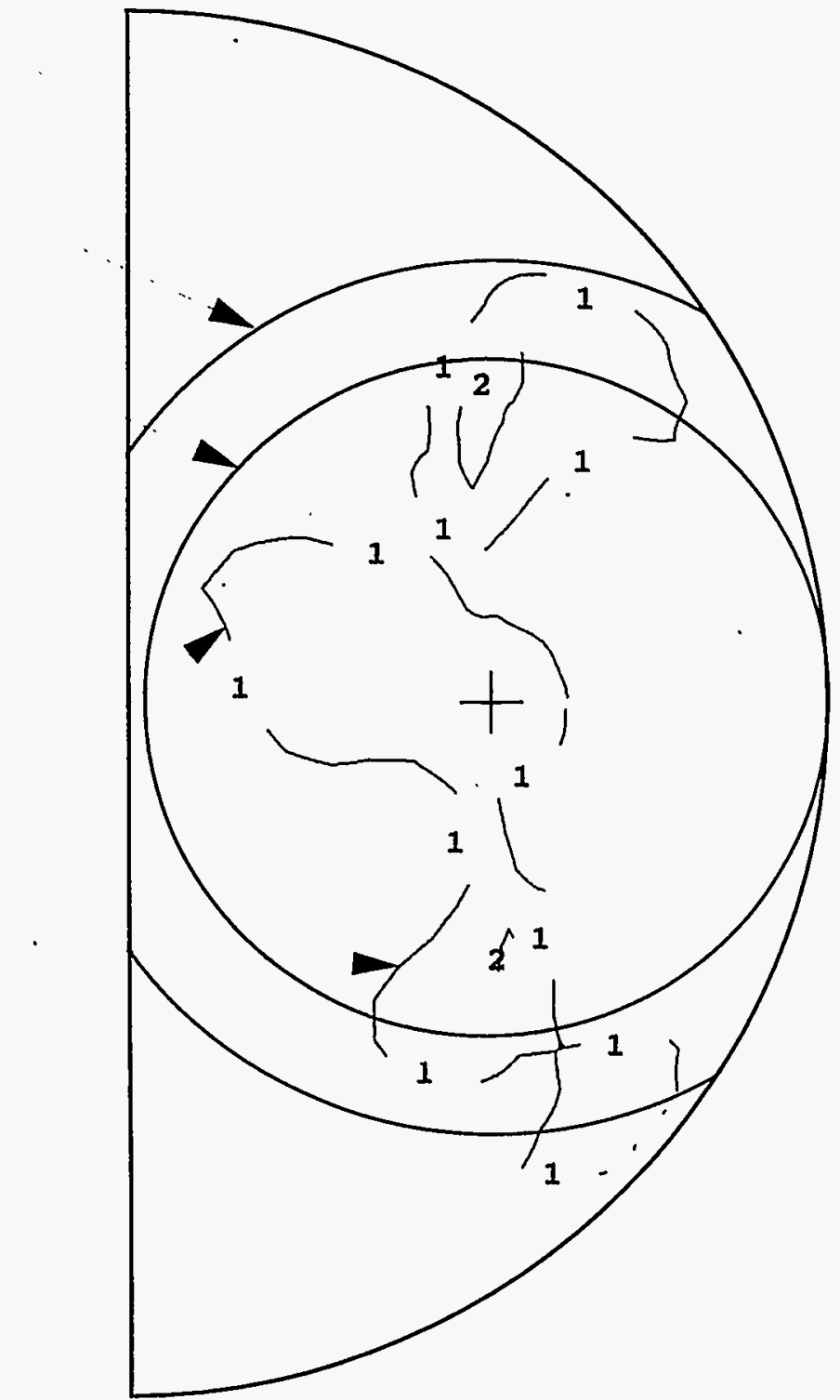

Velocity Magnitude Contours

$(1->0.2 \mathrm{~m} / \mathrm{s})$

$(2 \rightarrow 0.5 \mathrm{~m} / \mathrm{s})$

Figure C.83. Estimated Tank Bottom Areas for Possible Sludge Mobilization at Different Velocity and Yield Stress Values for Case 8 and 9 (30 ft/s jets) 
$0.100 \mathrm{~m} / \mathrm{s}, 6.5 \mathrm{~Pa}$

$r=9.5 \mathrm{~m}(31 \mathrm{ft})$

$0.125 \mathrm{~m} / \mathrm{s}, 10 \mathrm{~Pa}$

$\mathrm{r}=8 \mathrm{~m}(27 \mathrm{ft})$

$0.150 \mathrm{~m} / \mathrm{s}, 15 \mathrm{~Pa}$

$\mathrm{r}=7 \mathrm{~m}(23 \mathrm{ft})$

$0.175 \mathrm{~m} / \mathrm{s}, 20 \mathrm{~Pa}$

$\mathrm{r}=6 \mathrm{~m}(20 \mathrm{ft})$

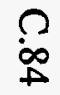

$0.200 \mathrm{~m} / \mathrm{s}, 26 \mathrm{~Pa}$

$\mathrm{r}=3 \mathrm{~m}(9 \mathrm{ft})$

Velocity Magnitude

Contours

$$
\begin{aligned}
& (1->0.100 \mathrm{~m} / \mathrm{s}) \\
& (2 \rightarrow>0.125 \mathrm{~m} / \mathrm{s}) \\
& (3 \rightarrow>0.150 \mathrm{~m} / \mathrm{s}) \\
& (4 \rightarrow 0.175 \mathrm{~m} / \mathrm{s}) \\
& (5->0.200 \mathrm{~m} / \mathrm{s})
\end{aligned}
$$

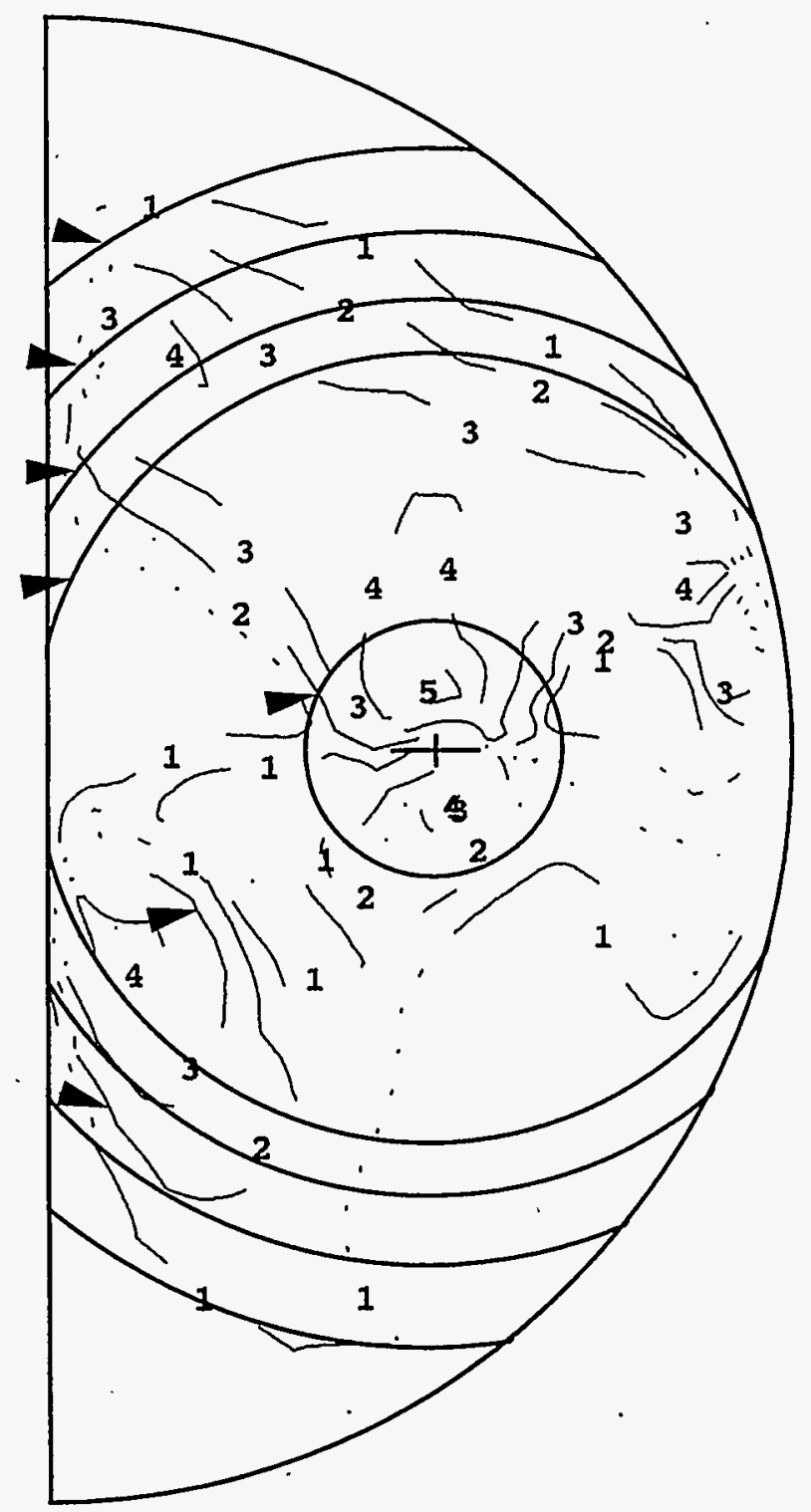

Figure C.84. Estimated Tank Bottom Areas for Possible Sludge Mobilization at Different Velocity and Yield Stress Values for Case 10 (15 ft/s jets) 


\section{Distribution}

No. of

Copies

Offsite

2 DOE Office of Scientific and Technical Information

2 Department of Civil and Environmental Engineering

Washington State University

Pullman, WA 99164

Attn: M. Hanaif Chaudhry

R. Y Itani

J. A. Cochran, Dean

Washington State University, Tri-Cities

Richland, WA 99352

M. Goldman

Laboratory for Energy-Related Health

Research

University of California at Davis

Davis, CA 95616

K. Kim

H. Krumb School of Mines

Columbia University

809 Seely W. Mudd

New York, NY 10027

R. B. Krone

Department of Civil and Environmental Engineering

University of California at Davis

Davis, CA 95616-5294

S. C. T. Lien, Director, EM-54

Office of Research and Development

Office of Technology Development

Office of Environmental Management

U.S. Department of Energy

Washington, D.C. 20585
No. of

Copies

J: F. Paul

Environmental Research Laboratory

U.S. Environmental Protection Agency

Naragansett, RI 02882

D. Robey

Waterways Experimental Station

U.S. Army Corps of Engineers

Vicksburg, MS 39180

A. Schneider

Massachussetts Institute of Technology

Department of Nuclear Engineering

Room 24-1098

77 Massachussetts Avenue

Cambridge, MA 02139

G. L. Schnoor

Center for Global and Regional

Environmental Research

Civil/Environ. Engineering Dept.

University of Iowa

Iowa City, IA 52242

J. E. Till, President

Radiological Assessment Corp.

Rt. 2, Box 122

Neeses, SC 29107

U.S. Department of Energy

Trevion II Building, EM-35

Washington, D.C. 20585-0002

Attn: J. Tseng

D. Pepson

Distr.1 
No of

Copies

2 U.S. Environmental Protection Agency Office of Radiation Programs $401 \mathrm{M}$ Street SW

Washington, D.C. 20460

Attn: R. Dyer

K. W. Yeh
No of

Copies

2 ICF Kaiser Hanford

A. N. Palit

S3-05

M. A. Przybylski

S2-47

87 Pacific Northwest National Laboratory

Onsite

10 DOE Richland Operations Office

D. H. Alexander

S7-51

S. T. Burnum

S7-53

R. F. Christensen

S7-54

J. J. Davis

S6-62

R. E. Gerton

C. A. Groendyke

G.A. Meyer

B. L. Nicoll

G. W. Rosenwald

W. R. Wrzesinski

26 Westinghouse Hanford Company

H. Babad

G. S. Barney

W. B. Barton

K. G. Carothers

J. L. Gilbert

J. P. Harris

D. L. Herting

J. D. Hopkins

G. D. Johnson .

J. W. Lentsch

J. M. Light

E. J. Lipke

D. A. Reynolds

C. A. Rieck (5)

G. J. Rust

M. J. Sutey

J. E. VanBeek (5)

R. J. Van Vleet
S7-30

T5-12

H5-21

R1-51

B4-08

S6-12

T6-09

R2-11

S7-15

S7-15

B4-08

S7-14

R2-11

R3-27

T4-01

T4-07

R3-27

H4-62
S. Q. Bennett

J. W. Brothers

B. C. Bunker

Y. L. Chen (3)

R. M. Ecker

A. R. Felmy

J. A. Fort

M. D. Freshley

P. A. Gauglitz

W. W. Laity

L. M. Liljegren

L. K. Holton

W. L. Kuhn

J. P. Lafemina

M. A. Lilga

J. Lui (3)

T. E. Michener

Y. Onishi (30)

F. E. Panisko

L. R. Pederson

M. R. Powell

R. K. Quinn

K. P. Recknagle (3)

D. R. Rector (3)

H. C. Reid

B. A. Reynolds

J. R. Rustad

W.D. Samuels

A. Shekarriz (10)

P. A. Smith (3)

C. W. Stewart

J. W. Virden

Information Release (7)
K7-90

K5-22

K2:25

K6-91

K9-77

K7-15

P8-34

P7-41

P7-27

K7-15

K9-73

K2-21

K2-25

P8-38

K2-44

K7-15

K9-33

P8-34

K2-44

P7-14

K9-69

K7-15

K7-15

K7-15

P7-19

K9-77

K2-44

K7-15

K2-44

K7-15

K2-44

K1-06

Distr.2 